\title{
KANGAROO MOTHER CARE
}

\author{
PARTICIPATORY ACTION RESEARCH WITHIN A
}

NEONATAL INTENSIVE CARE UNIT IN

AOTEAROA NEW ZEALAND

Rebecca Jane Bear

\begin{abstract}
A thesis
submitted to the Victoria University of Wellington

in fulfilment of the requirements for the degree of

Doctor of Philosophy in Health
\end{abstract}

Victoria University of Wellington

2019 


\begin{abstract}
Human infants share common biological and developmental needs in the postnatal period that are optimally met during intimate contact with their mother or primary caregiver. In the case of infants hospitalised in tertiary-level neonatal intensive care units (NICUs), there is a departure from instinctual caregiving and nurturing found in the mother-infant pair, due in part, to a model of care which supports maternalinfant separation. This can lead to suboptimal physiological responses, altered neurobiology and life-long negative health effects. The social construction of neonatal care currently positions it within the paradigm of biomedical science. Where family-centred, developmental care frameworks have been integrated, and Kangaroo Mother Care (KMC) has been embedded into caregiving routines, enhanced patient, whānau/family, staff and organisational outcomes have been found.
\end{abstract}

This study is underpinned by the importance of KMC for the enhancement of infant and whānau/families' health and developmental outcomes. Despite its classification as an evidence-based practice, and recommendations by the World Health Organisation for its use in all healthcare settings, KMC is inconsistently applied. The highly complex and contextual nature of the environments where medicallydependent babies are cared for is acknowledged. There is a need for health services to explore innovative research approaches, through a social science lens, to assist in the implementation of KMC. This thesis illustrates one such approach.

The purpose of this study was to explore and activate improvement of the KMC programme within one NICU in Aotearoa New Zealand using Participatory Action Research (PAR). The research was theoretically informed by Als' developmental biology and care theories, D'Agata's Infant Medical Trauma model, and the Foucauldian concept of power/knowledge through a critical feminist lens. A participatory approach was chosen in the hope that transformation of KMC practice would be achieved and embedded within this NICU. In addition, I intended to contribute to the emerging body of evidence calling for the collaboration of all community members toward enhanced quality of KMC. Multiple methods were used 
to capture data relating to the NICU's KMC programme through audit, observation and interview of key stakeholders.

Project planning included the conventions of PAR generally applied to research using this methodology. Three iterative cycles of exploration, implementation and evaluation of the KMC programme were envisaged within this setting. Active participation with multiple NICU stakeholders was planned for, forming the basis of action-based change and improvement of KMC. However, the three-cycle process was not achieved within the time limitations of my research, with field work finishing at the conclusion of the first exploratory cycle. This thesis describes the unfolding processes of PAR, as well as the inclusion of a secondary discourse analysis and parental perspectives from local and global literature.

Key findings showed inconsistently documented KMC and the near-absence of KMC practice for a significant group of babies. Whilst the benefit of KMC was embedded in the understanding of participants, this knowledge did not translate to practice. There was an unrealistic optimism about the functioning of KMC by most of the stakeholders. In addition, participants expressed ambiguity about their programme, contributing to and influenced by suboptimal KMC education and training. A pathway to improvement of their KMC programme was lacking, and the lines of responsibility for it were unclear. This factor undoubtedly contributed to the difficulties of implementing a full PAR project. Whilst parental, staff and organisational factors were found to influence KMC implementation, arguably the greatest effect on the intervention were the power relations inherent within the normative technocratic, biomedical paradigm. Power relations constituting what was considered authoritative knowledge, and who was authorised to speak, impacted on the participatory nature of the research itself. This resulted in the research not proceeding past the first PAR exploratory cycle through to rounds of implementation and evaluation.

This thesis describes participatory inquiry into one KMC programme in the highincome NICU setting, through the lenses of multiple participants within the context of Aotearoa New Zealand. These were not previously known. It also provides an example of how Foucauldian- and feminist-informed PAR methodology may be 
used within the NICU setting for inquiry into KMC, an intervention positioned outside of the normative biomedical framework.

Keywords: Kangaroo Mother Care, developmental care, mother-infant attachment, neonatal intensive care unit, participatory action research, Foucauldian discourse analysis, feminist theory, maternal voice. 


\title{
DEDICATION
}

\author{
For MotherBabies and their Whānau
}

May the loving bonds be nurturing, supportive, and endure for a lifetime 


\section{ACKNOWLEDGEMENTS}

It is with great delight that I honour and give thanks to my three precious children, Ellie, Thomas and Bella, without whom I would not have dreamed to start or complete this work. The seeds were planted in the moments that I became your Mother. Your part to play in my development into a mature woman, where I have found the strength and courage to become some of who I am meant to be, cannot be understated. Kids, I understand that you won't get the enormity of this at your tender age, nonetheless, I assure you that this thesis is as much yours as it it mine. Thank you.

To Mum, Dad, Jo, Sal and the rest of the colourful group who are my First Family. Our shared experiences form my cells and bones and I am so grateful to carry you wherever I go. I would not trade a single moment - laughter nor tears. Now, more than ever, I understand the value of all that we have shared.

To my dear friends, my second and Soul Family, you are more support than you know. Jude, Nic, Eija, Gerry and Brown, whether I was fully able to articulate it or not, I could not have pushed through to the summit had it not been for the wines, the walks and the innumerable cups of coffee. The wisdom contained in every precious moment with you all is the beating heart of my friend-privileged life. A million thank-yous don't add up to the love I have for you extraordinarily kind-hearted folk. Joan Skinner and Robyn Maude, the term 'supervisor' pays no homage to the place in my heart where you now reside. Joan, your strength and tenacity, your courage and your often unexpected truth-telling have modelled the traits of the wise crone I intend to become. Robyn, you are 100\% authenticity, owning your spaces as midwife, academic, leader and all-round Australian powerhouse. Thank you, women. Thank you for modelling the values and characteristics of the leader I aspire to one day be.

From the fabulous female supervisors of my doctoral journey, to the epitome of firm and gentle mentorship from a former postgraduate life, David Mellor. It was your giant shoulders from which I launched this project. You have been inspiring me in 
one way or another (academically and otherwise) for 25 years, well over half of my life. I borrowed my confidence from you. Deep gratitude is mine.

To the vivacious and lively bunch who gather around the lunch table of the humble offices at the Graduate School of Nursing Midwifery and Health: short story, you're the best! Many a needed laugh I have had at that table - the best medicine of all. Two special mentions. Helen Rook: the absence of that tearful chat back in the beginning of 2017 may have completely derailed the laboured efforts of my research, for some time at least. And to Jeanie Douché: I am astounded by your grace and Foucauldian/feminist wisdom. Thank you for your generosity in teaching the class 'MF, 101 - for dummies'.

Next, to the final and no less important pillar of my support crew, the wonderful people of the ShoreLine Cinema. The emotional, financial and playful role you have held in my life as I've climbed Mount PhD were hugely foundational. You have all provided enormous strength and a delightful place to rest when needed - and it was often needed! Thank you for helping to keep the wheels turning.

Now, to the staff and participants from one very special Neonatal Intensive Care Unit. Quite clearly, I couldn't have done it without you. It seems no less important to thank the precious little bundles for whom the NICU was created, because conscious or not, you are the stars of the show. My enduring prayer for you all is this: may the love of your parents and the good people of the NICU community continue to bloom and grow over your lifetime. You are all the strongest warriors I know.

No less, and finally, it is with thanks to the power that I call God, including the church of Zumba, that I have found the grace and humility to commit to my life's work through the effort on these pages. I am truly blessed. 


\section{TABLE OF CONTENTS}

ABSTRACT

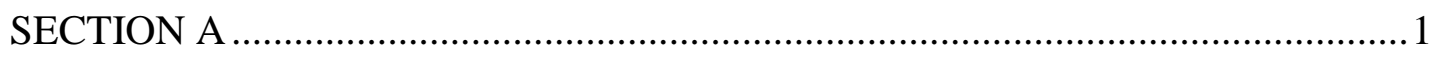

CHAPTER ONE: INTRODUCTION ............................................................ 1

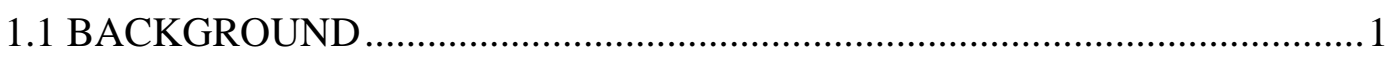

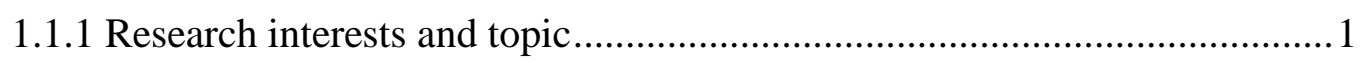

1.1.2 Neonatal intensive care in the context of Aotearoa New Zealand...............4

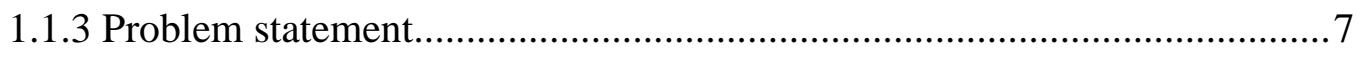

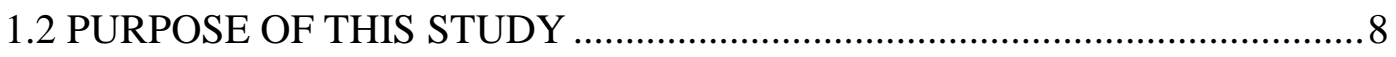

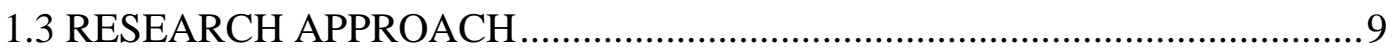

1.4 POSITIONALITY: 'I', THE RESEARCHER …............................................ 11

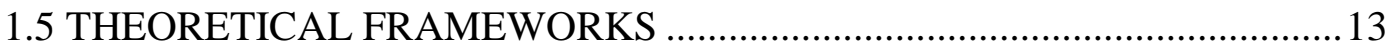

1.5.1 Developmental biology of the infant, mother and dyad .......................... 13

1.5.2 Developmental care of the infant in the NICU ....................................... 14

1.6 THESIS OVERVIEW AND ORGANISATION ….................................. 18

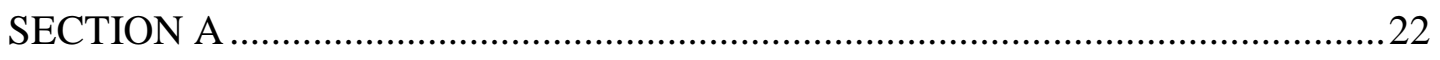

CHAPTER TWO: LITERATURE REVIEW …...................................................2

2.1 REVIEW ONE: INFANT DEVELOPMENTAL BIOLOGY ........................24

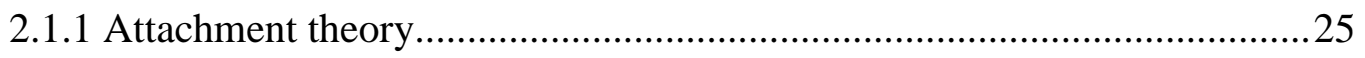


2.1.2 Biological expectation in the postnatal period .28

2.1.3 Biological embedding of early adverse experience .................................30

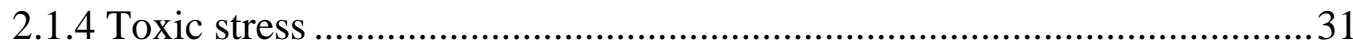

2.2 REVIEW TWO: DEVELOPMENTAL CARE AND KMC IN THE NICU ...34

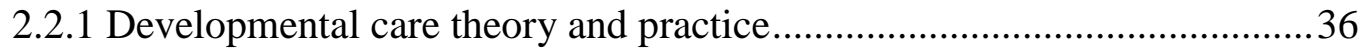

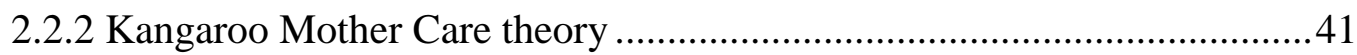

2.3 REVIEW THREE: IMPLEMENTATION OF KMC IN THE HIGH-INCOME

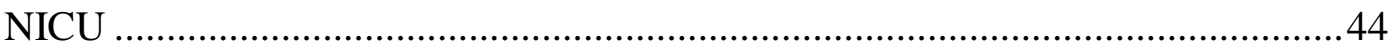

2.4 REVIEW FOUR: SOCIAL CONSTRUCTION OF NEONATAL INTENSIVE CARE \& INSTITUTIONAL RACISM …..................................................... 49

2.4.1 History of neonatal intensive care …...................................................50

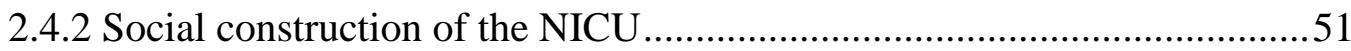

2.4.3 Institutional racism in New Zealand .....................................................56

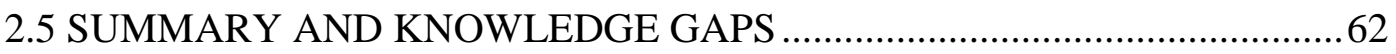

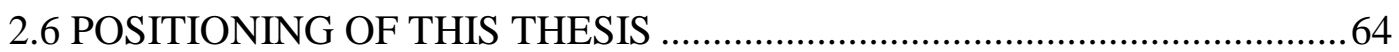

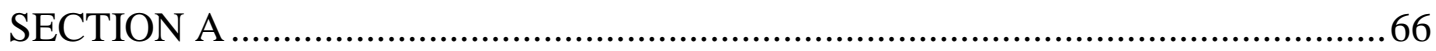

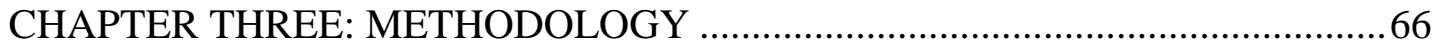

3.1 PARTICIPATORY ACTION RESEARCH ................................................67

3.1.1 Philosophical underpinnings of PAR .................................................. 70

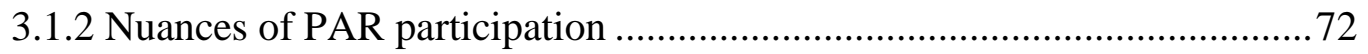

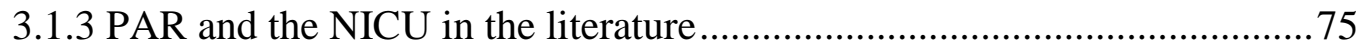

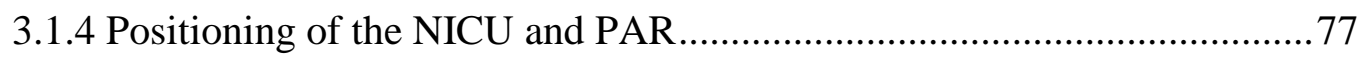

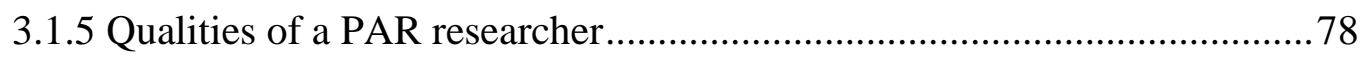

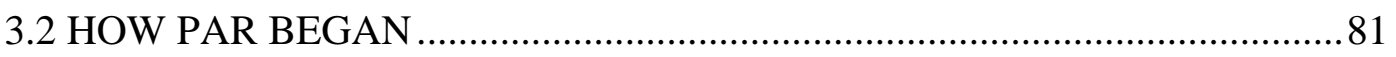

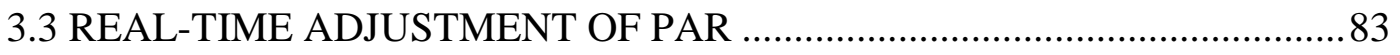

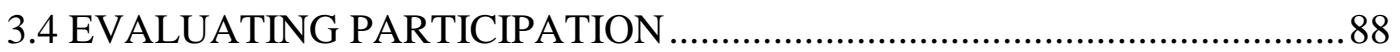

3.5 GOING DEEPER: FEMINISM AND FOUCAULT ....................................92

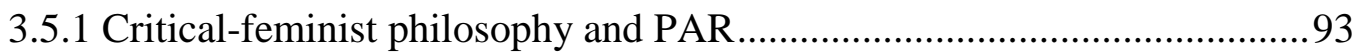




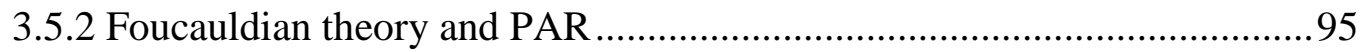

3.6 TRUSTWORTHINESS AND VALIDITY ..................................................96

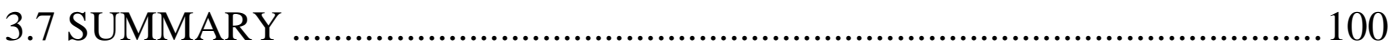

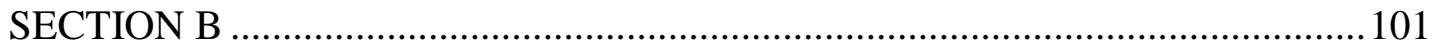

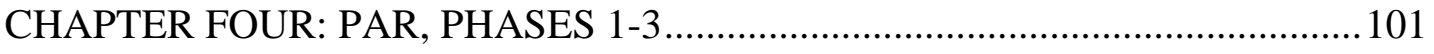

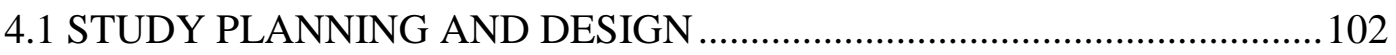

4.1.1 Research setting and pre-project planning ......................................... 102

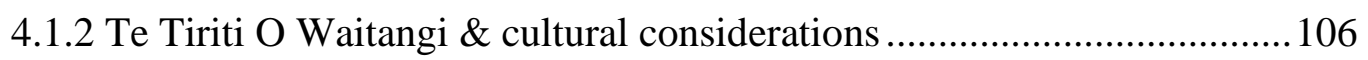

4.1.3 General ethical considerations for PAR approaches............................. 107

4.1.4 Ethical considerations, gatekeepers and champions ............................. 108

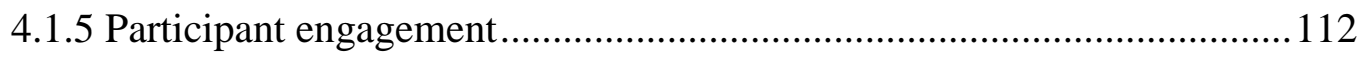

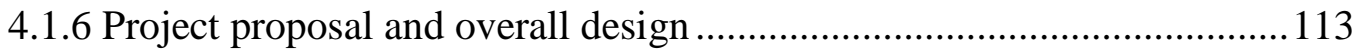

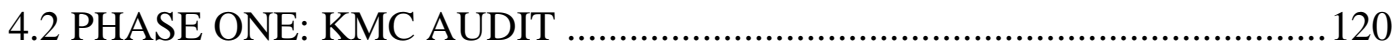

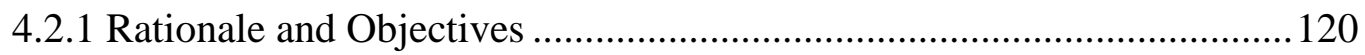

4.2.2 Participant Engagement and Research Ethics....................................... 121

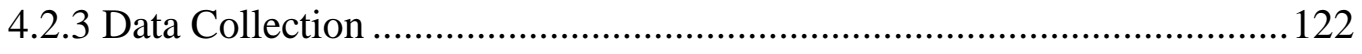

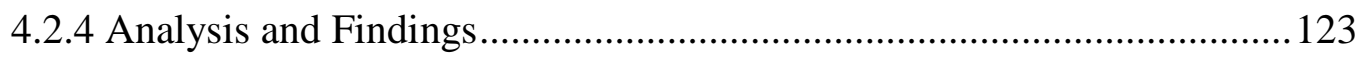

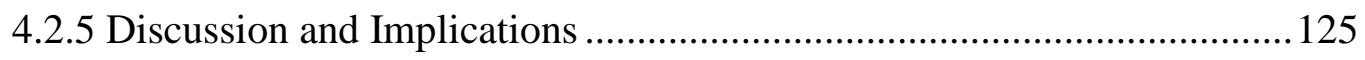

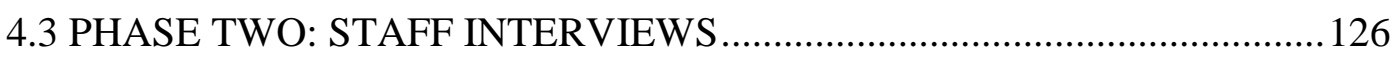

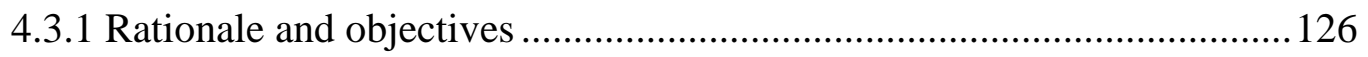

4.3.2 Participant Engagement and Research Ethics..................................... 127

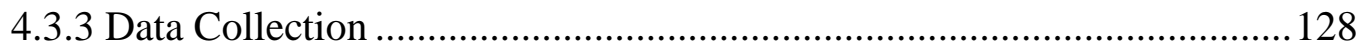

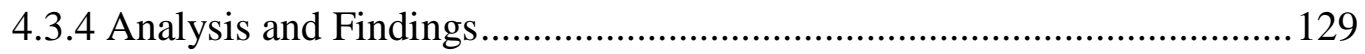

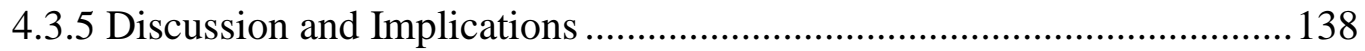

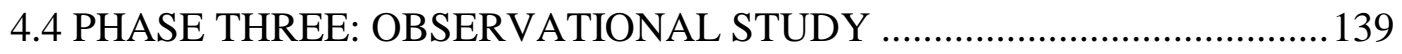

4.4.1 Rationale and Research Questions................................................... 140

4.4.2 Participant Engagement and Research Ethics..................................... 140 
4.4.3 Data Collection 142

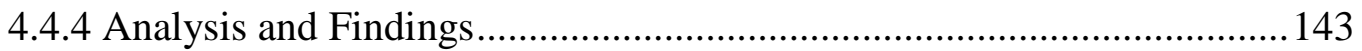

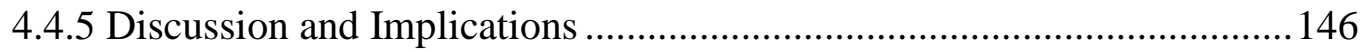

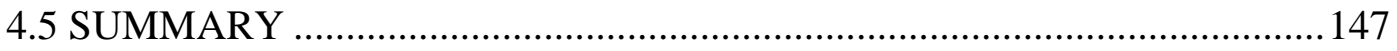

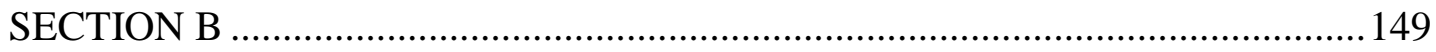

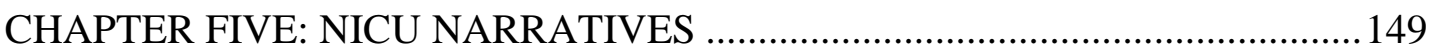

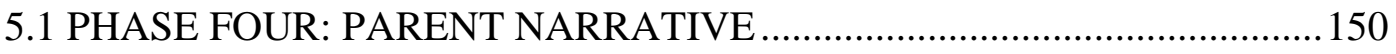

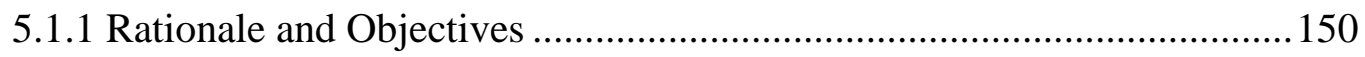

5.1.2 Participant Engagement and Research Ethics..................................... 151

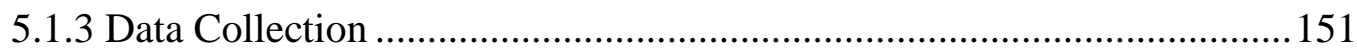

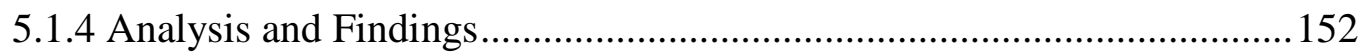

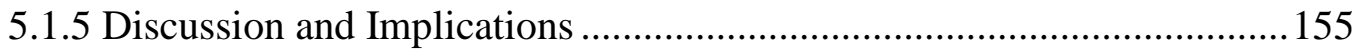

5.2 NICU NARRATIVES: 'OTHER’ VOICES ............................................... 157

5.2.1 Whānau Māori voices from the NICU .................................................... 157

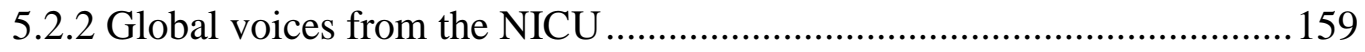

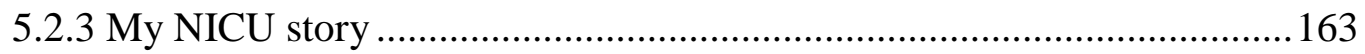

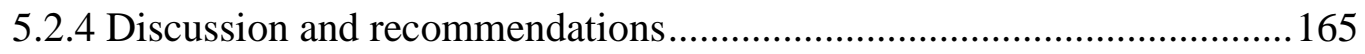

5.3 PHASE FIVE: PARTICIPANT-FACILITATOR NARRATIVE................... 166

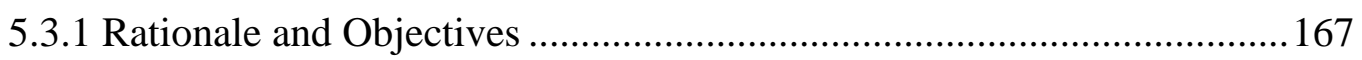

5.3.2 Participant Engagement and Research Ethics....................................... 167

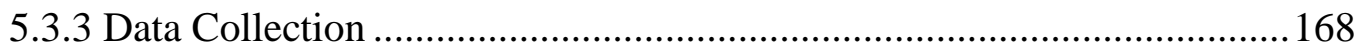

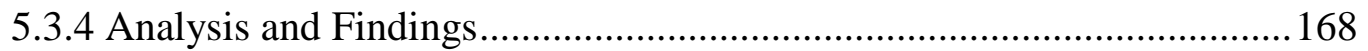

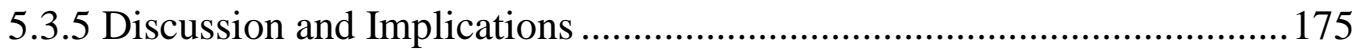

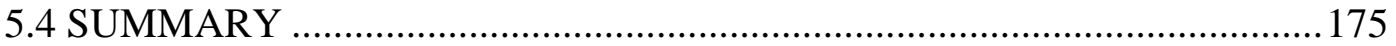

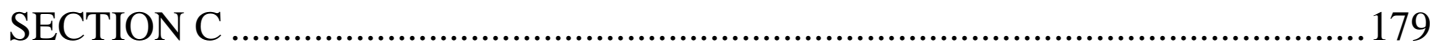

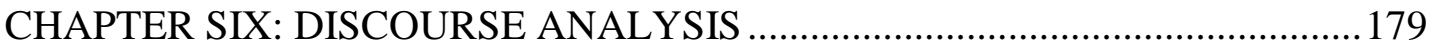

6.1 DISCOURSE ANALYSIS, FOUCAULT AND FEMINIST THEORY .......180 
6.3 DISCOURSE ANALYSIS IN THE NICU ............................................... 183

6.3.1 Genealogy of medicine, maternity and infant care .............................. 183

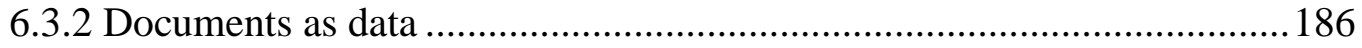

6.4 THE DISCOURSE ANALYSIS PROCESS .............................................. 187

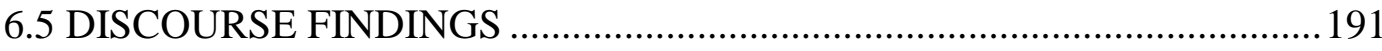

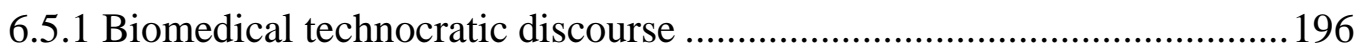

6.5.2 Health System Discourse ...................................................................2200

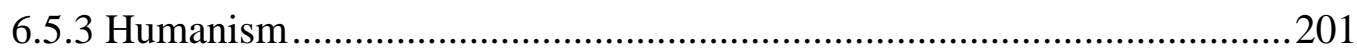

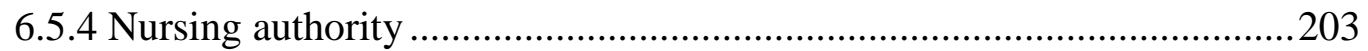

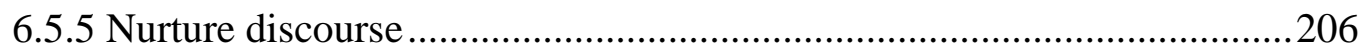

6.6. DISCURSIVE ABSENCES AND SILENCES .......................................210

6.7 COUNTER-DISCOURSES AND RESISTANCES .................................214

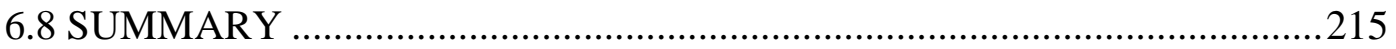

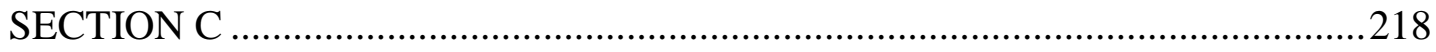

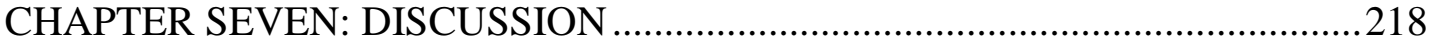

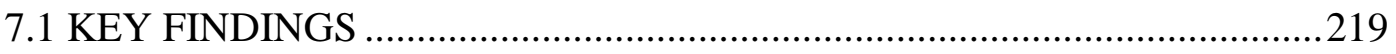

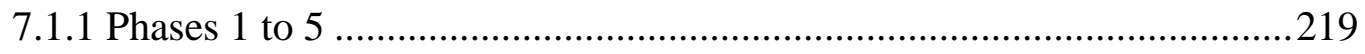

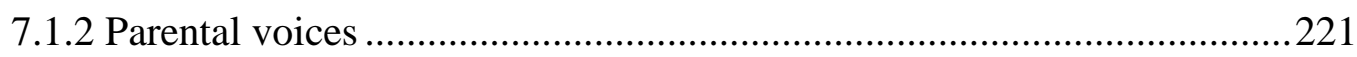

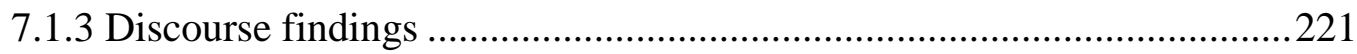

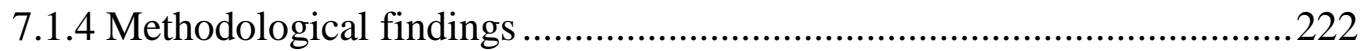

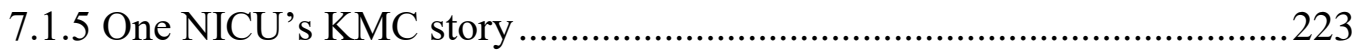

7.2 CONTRIBUTION TO KMC KNOWLEDGE .........................................227

7.3 PAR CONTRIBUTIONS AND REFLECTIONS ....................................228

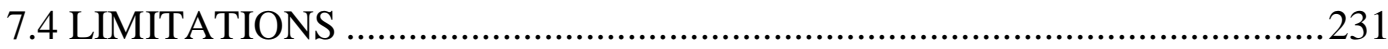

7.5 RECOMMENDATIONS BASED ON THIS STUDY …...........................232

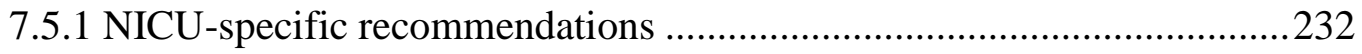




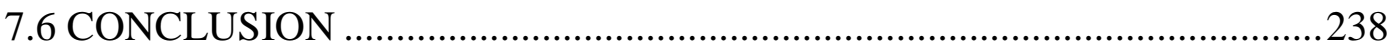

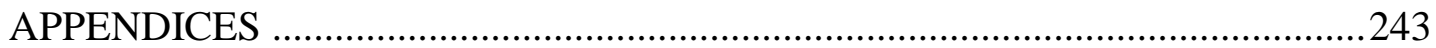

Appendix 1: Health and Disability Ethics Committee, Approval 2 .....................243

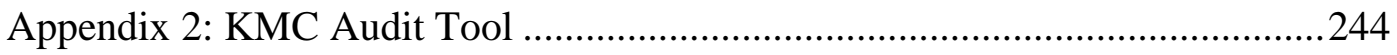

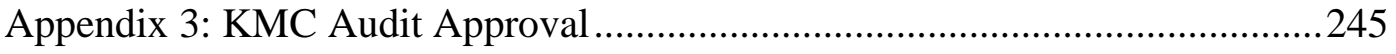

Appendix 4: Human Ethics Committee Approval.............................................248

Appendix 5: Participant Information Sheet (Staff interviews, phase 2)..............249

Appendix 6: Participant Consent Form (Staff interviews, phase 2) ....................252

Appendix 7: Staff Interview Guide (phase 2) ...................................................2. 253

Appendix 8: Human Ethics Committee Approval, Amendment 1......................254

Appendix 9: Human Ethics Committee Approval, Amendment 2.....................255

Appendix 10: Institutional Approval KMC Observation (phase 3) .....................256

Appendix 11: Staff Information Sheet (KMC observation, phase 3) ...................257

Appendix 12: Parent Information Sheet (KMC observation, phase 3) ................260

Appendix 13: Participant Consent Form (KMC observation, phase 3) ...............262

Appendix 14: KMC Observation Tool (phase 3) ..............................................263

Appendix 15: Human Ethics Committee Approval, Amendment 3 ....................264

Appendix 16: Participant Information (Parent interview, phase 4).....................265

Appendix 17: Participant Consent Form (Parent interview, phase 4) ..................268

Appendix 18: Parent Interview Guide (phase 4) ..............................................2. 269

Appendix 19: PAR-Participant Interview Guide (phase 5) [abridged] ................2270

Appendix 20: Health and Disability Ethics Committee, Approval 1 ..................227

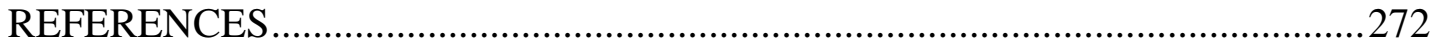




\section{LIST OF TABLES}

Table 1: Ladder of Pretty......................................................... 73

Table 2: Comparison of Participation Levels Against the Ladder of Pretty...........91

Table 3: Proposal for KMC Quality Improvement Research................................114

Table 4: Summary of KMC Audit Statistics................................ 122

Table 5: Self-identified Roles of Staff Interview Participants................... 127

Table 6: Summary of Themes from SWOT Analysis.........................130

Table 7: Summary of Staff Suggestions for KMC Quality Improvement Projects..136

Table 8: Observation Statistics for Predischarge Infants........................ 143

Table 9: Example of the Narrative Analysis Framework (phase 4)........................152

Table 10: Example of the Narrative Analysis Framework (phase 5).....................168

Table 11: Tenets of Three Medical Models, Adapted for the NICU............... 188

Table 12: Summary of Discourses and Themes.....................................192-194

Table 13: NICU-specific Recommendations for Quality Improvement of KMC.

\section{LIST OF FIGURES}

Figure 1: Timeline and Overview of the Study............................. 18

Figure 2: Search Strategy Algorithm of Developmental Care and KMC in the NICU. .35

Figure 3: A Simple Representation of PAR ......................................................69

Figure 3: PAR Cycle One, Exploration of KMC ....................................................115

Figure 5: From NICU to NIPU - a Paradigmatic Reframe....................................2.23 


\section{ABBREVIATIONS}

$\begin{array}{ll}\text { ACE } & \text { Adverse childhood experience } \\ \text { DC } & \text { Developmental care } \\ \text { DHB } & \text { District health board } \\ \text { FCC } & \text { Family-centred care } \\ \text { FDA } & \text { Foucauldian discourse analysis } \\ \text { HEC } & \text { Human ethics committee } \\ \text { IMTN } & \text { Infant Medical Trauma in the NICU } \\ \text { KC } & \text { Kangaroo Care } \\ \text { KMC } & \text { Kangaroo Mother Care } \\ \text { PPP } & \text { Preliminary planning phase } \\ \text { NICU } & \text { Neonatal intensive care unit } \\ & \\ & \text { Participatory action research } \\ \text { PAR } & \\ & \end{array}$




\section{SECTION A}

\section{CHAPTER ONE: INTRODUCTION}

\subsection{BACKGROUND}

\subsubsection{Research interests and topic}

The main focus of this research, quality improvement of Kangaroo Mother Care is underpinned by knowledge of human infant development, developmental care theory, and the support of maternal-infant attachment within the hospital setting. In addition, this study highlights the use of methodologies which support collaborative research within complex healthcare environments and the translation of evidencebased knowledge into practice. This thesis is the culmination of three years of a participatory action research (PAR) project conducted within one neonatal intensive care unit (NICU) community, with particular focus on the intervention of Kangaroo Mother Care (KMC). The majority of infants who are hospitalised in the NICU are either preterm or low birthweight, representing a group who require increased healthcare support in the short term, and for some, over their entire life course. The plight of the preterm baby, both individually and collectively, cannot be understated. For these babies, prematurity represents a major traumatic episode in their lives and in the lives of their parents and their wider whānau/family. From a physiological perspective, premature birth is a 'brain changer' for the infant (Coughlin, 2017). That is, maternal separation and some environmental aspects of the NICU impact on the developing brain in profound and often negative ways, and possibly for life (Cho et al., 2016; Fox, Levitt, \& Nelson III, 2010). The likelihood of longlasting negative effects of sub-optimal bonding on the infant, mother, and family, are also greatly increased by this event (Kommers, Oei, Chen, Feijs, \& Oetomo, 2016). Cognitive and psychosocial indices such as difficulty integrating into school, increased relational and behavioural difficulties and lowered intellectual capacities are more likely for the child born preterm (Feldman, Rosenthal, \& Eidelman, 2014). As well as the individual health needs of preterm babies, mothers and whānau/families, an enormous public health burden has emerged worldwide as a result of increasing 
prematurity and survival of babies at increasingly lower gestations (Sharma, Murki, \& Oleti, 2016a). Implementation of safe, evidence-based interventions and care frameworks which support the development of hospitalised infants and their whānau/families, in a timely and cost-effective way, are urgently required. One such intervention, Kangaroo Mother Care, has a compelling evidence base to recommend it (Conde-Agudelo \& Diaz-Rossello, 2016). When implemented, KMC has been shown to reduce mortality and physiological and psychosocial morbidities in a variety of different settings (Boundy et al., 2015).

When used to its fullest extent, Kangaroo Mother Care involves placing a naked newborn infant into prolonged and continuous skin-to-skin contact on their mother's bare chest as soon as possible after birth. Additional goals of KMC are exclusive breastfeeding, timely (ideally, early) discharge from the health facility, and supportive follow-up at home (Chan et al., 2016; Charpak et al., 2017). The fourphase 'intervention', in this form, was first used as a developmental care method in the late 1970's by Drs Rey and Martinez in Bogota, Columbia where the use of incubators for traditional care of the infants was either unavailable or unsafe. More commonly however, it is one component of KMC, skin-to-skin contact (SSC), that is being measured and reported within the literature from high income countries for both pre- and full-term babies (Bergh et al., 2016; Bergh et al., 2012b; Bergh, van Rooyen, \& Pattinson, 2008; Chan 2016, Nyqvist et al., 2010b; Nyqvist \& Heinemann, 2011). The KMC intervention in its entirety is found to be insufficiently applied in most global settings (Bergh, Charpak, Ezeonodo, Udani, \& van Rooyen, 2012a; Chan, Labar, Wall, \& Atun, 2016; Smith, Bergelson, Constantian, Valsangkar, \& Chan, 2017). The findings of one recent systematic review revealed that " $\mathrm{SSC}$ is accepted in research and programmatic settings as an essential component of $\mathrm{KMC}$, but the other components vary by context..." (Chan et al., 2016, p7).

The positive effects of Kangaroo Mother Care are now accepted as empirical science, "an evidence-based approach to reducing mortality and morbidity in preterm infants" (Seidman et al., 2015, p. 1). Scientific evidence exists for uninterrupted skin-to-skin contact at birth for the majority of infants (Lorenz et al., 2017; Phillips, 2013). It is accepted as a developmentally appropriate way of meeting infant and maternal physiological, neurological and psychosocial needs at a sensitive stage of 
their development (Boundy et al., 2015; Conde-Agudelo \& Diaz-Rossello, 2014; Conde-Agudelo \& Diaz-Rossello, 2016). Consensus exists amongst key stakeholders in neonatal health that "the uptake of KMC practice and coverage of KMC services have not progressed well in many countries" (Bergh et al., 2016, p. 2) and that "KMC should be adopted and accelerated as standard of care as an essential intervention for preterm newborns" (Engmann, Wall, Darmstadt, Valsangkar, \& Claeson, 2013, p. e26). The successful and sustained implementation of the entire KMC package requires developmentally supportive care frameworks that consider the contexts of the individuals and institutions involved. Evaluating and providing structure for ongoing assessment of the baby and whanau/family, the healthcare environment and the caregiver capacity are required. Principles of developmental care are commonplace, yet systematic implementation is rarely attained (Atun-Einy $\&$ Scher, 2008).

I was interested in developing an in-depth understanding about the functioning of KMC practice in one NICU in my own country, for contribution to the local and global field of research. For the purposes of the study, I consistently adopted the phrase Kangaroo Mother Care (KMC) to represent the intermittent skin-to-skin contact which is commonplace in affluent settings, as described by Nyqvist et al. (2010) and an Expert Group of the International Network on Kangaroo Mother Care (p. 1):

the method $[\mathrm{KMC}]$ is implemented as limited sessions with mother-infant SSC [skin-to-skin contact] in KP [kangaroo position], such as one or a few hours, not necessarily every day, occurring over a limited period.

Whilst I acknowledge that the practice of KMC in this more limited way is usually termed kangaroo care $(\mathrm{KC})$ or skin-to-skin care/contact, I had a clear rationale for upholding the KMC term (Nyqvist et al., 2010). Firstly, the research involved the possibility of practice transformation to more extensive use of KMC and therefore I felt it was important to use language which would hold this vision. Secondly, initial interactions with NICU staff members revealed the embedded use of KMC as common terminology, amongst a raft of other descriptors for the intervention, and I therefore wanted to align with the unit's common language. Lastly, I was informed by feminist and holistic worldviews which meant that I worked to keep women connected to the intervention through language that acknowledged their importance as mothers to their babies. In doing so, I contest that I didn't further separate and 
marginalise women by absenting the word 'mother' from the healthcare discourse relating to KMC. A broad historical and social perspective of neonatal intensive care units in Aotearoa New Zealand supports the situation of this study for the reader.

\subsubsection{Neonatal intensive care in the context of Aotearoa New Zealand}

Recent maternity statistics show that over $95 \%$ of babies are born in primary, secondary or tertiary maternity units across Aotearoa New Zealand (MOH, 2017).

Many new mothers and babies are healthy and discharged home to their whānau within days. However, some infants do require specialist medical care within a level three Neonatal Intensive Care Unit for reasons such as prematurity, low birth weight and a raft of congenital and medical issues.

Data and information relating to NICU hospitalisation of babies in Aotearoa is fairly scant and lacking uniformity between organisations who collect it (Pihama, 2010). Since 1994, the Australian and New Zealand Neonatal Network (ANZNN) have collated data from secondary and tertiary NICUs from both countries and more recently, Singapore as well. The purpose of the ANZNN is to provide a collaborative audit that can steer improvements in NICU care. Repeated audits have captured statistics from babies born at less than 32 weeks, weighing less than $1500 \mathrm{~g}$, or receiving assisted ventilation, major surgery and/or therapeutic cooling (ANZNN, 2018). Whilst the reports do not constitute an exhaustive picture of NICU care and all mother-baby dyads in Aotearoa, they are helpful in providing some context for this study. Notable limitations of the data include a dearth of ethnicity and social deprivation trends, exclusion of babies born at more than 32 weeks gestation, and the use of aggregated data from three different country contexts.

Anecdotally, prematurity (with or without low birthweight) is the most common single reason for admission to tertiary NICUs. Recent maternity clinical indicators from 2015 and 2014 show a relatively stable nationwide prematurity rate of $7.5 \%$ and $7.4 \%$, respectively across the population ( $\mathrm{MOH} 2014,2015)$. While it remains unknown what proportion of the 4,500 babies born prematurely each year are admitted to NICUs across the country, it is assumed that the majority of preterm babies are admitted. Ministry of Health maternity statistics most relevant to NICU admissions, centre around maternal ethnicity and age and infant birthweight. Around $6 \%$ of all babies born between 2006 and 2015 were of low birthweight and were 
described according to ethnicity, maternal age and deprivation quintile (MOH 2017). According to the most recent statistics, babies born in Aotearoa with low birthweight are more likely when (MOH 2017, p. 56):

i. Babies are born to Māori or Indian women (6.7\% and 9.6\%, respectively)

ii. Maternal age is over 40 or under 20 ( $8.5 \%$ and $7.0 \%$, respectively)

iii. They are from more deprived neighbourhoods (6.5\% from quintile 5 areas, highest deprivation).

Interestingly, rates of prematurity varied across district health board (DHB) regions, with West Coast and Southland DHBs over-represented and the Northland DHB under-represented, when compared with the national rates $(9.2 \%$ and $6.6 \%$, respectively (MOH, 2015).

For most premature babies born in Aotearoa, including those born at the lowest survivable gestations, the chance of their survival to discharge home has steadily increased over the last two decades: "New Zealand currently has one of the best survival rates for premature infants in the world" (MOH 2005, p. 1). More recently, New Zealand researchers reported comparatively high-quality care in our tertiary NICUs for 23- and 24-week gestation babies, concluding that (Berry et al., 2017, pp. 6-7):

survival free of moderate or severe disability can be achieved in infants born at 23 weeks gestation... [and] our high rate of resuscitation in infants born at 23 weeks did not result in a disproportionately high burden of severely neurologically compromised infants.

A second publication from the same New Zealand study foregrounded the association between gestations of less than 39 weeks and poorer outcomes involving mortality, health, education and social factors, when compared with full-term babies. The researchers urged that poorer outcomes, persisting at least until adolescence, required investigation into "The additional impact of environmentally modifiable factors (such as adequacy of housing stock) on those born preterm" (Berry et al. 2018, p. 8).

The latter point, that of wider sociocultural determinants affecting babies and mothers, brings me to recent concerning evidence pertaining to New Zealanders, presented by the Health Quality and Safety Commission in 2018. Whilst the Ministry 
of Health statistics (2015) reported liveborn premature babies with "no obvious trends... except a higher proportion among babies born to older women” (p. 58), neonatal mortality figures showed an alarming trend with respect to ethnicity and racial inequality in Aotearoa. The twelfth annual report of the Perinatal and Maternal Mortality Review Committee (PMMRC, 2018) focused on neonatal death statistics that haven't declined in the period from 2007 to 2016. The report described prematurity as the most common cause of death between 20 weeks of gestation and the first 27 days after birth, with babies more likely to die if their mothers were of Māori, Pacific or Indian ethnicity. An analysis of persistent racial inequities for women delivering babies who were extremely preterm was provided in the PMMRC report (2018, p. 8):

More babies of Māori, Pacific and Indian mothers are born extremely preterm and so these ethnic groups are disproportionately affected by suboptimal care for mothers and babies at these gestations. This is compounded if there are also inequities in provision of care by ethnicity.

The in-depth analysis identified inequities by ethnicity and by maternal age $(<20$ years) in a number of care areas affecting neonatal survival. These related to access to antenatal care, access to tertiary neonatal facilities, treatment with antenatal corticosteroids, and attempted resuscitation at extreme preterm gestations. An analysis of care pathways was not undertaken. However, inequities by ethnicity are increasingly found in health care both in New Zealand and overseas, and are associated with implicit bias and racism.

It was sociodemographic and qualitative data most relevant to the experiences of the NICU community in one tertiary setting that were of most interest for my study. This may have included information such as common care practices (developmental care, KMC practice, family-centred care) and perspectives from staff and consumers relating to their lived experiences of hospitalisation. Statistics collected from babies receiving care in this NICU included: infant gestation, birthweight, birth place, date of admission/discharge, gender, length of hospital stay, discharge destination, home address, delivery method and Apgar scores at 1 and 5 minutes after birth. Maternal date of birth and ethnicity were also collected. In line with the nationwide situation, there was no data available from within this NICU context which addressed wider determinants of wellbeing for infants and their whānau.

Importantly, there was a near-absence of literature relating to indigenous perspectives of NICU care, with just two reports representing "Māori experiences from a Māori perspective" (Thompson, 2009, p. 9). One kaupapa Māori researcher 
concluded after a review of the literature pertaining to Māori experience of the NICU that "the gaps that exist in available literature relating to Māori families of premature infants was brought to light" (Thompson, 2009, p. 9). A decade later, that deficit appears just as large and just as important, given that "the disparities in infant mortality experienced by indigenous women worldwide reinforce the need to understand an indigenous experience in the NICU (Thompson, 2009, p. 31).

In addition, I was only able to find one reference in the broader literature relating to neonatal care frameworks in Aotearoa, with the Ministry of Health (2005) stating (p. 28):

DHBs need to be identifying appropriate models of care... achievable in the current neonatal environment... [which] in the future may require increasing involvement of parents in neonatal care... DHBs need to work with each other in identifying capital needs and options that may exist for collaborating on projects.

\subsubsection{Problem statement}

Within the NICUs of high income countries such as Aotearoa New Zealand, a traditional neonatal care paradigm that results in early and enduring maternal-infant separation and inconsistent application of KMC may predominate (Abadia-Barrero, 2018; Nyqvist, 2016). This perspective is speculative, given that no empirical evidence exists at this time to support or refute the idea. Statistics for KMC are not currently published by the New Zealand Ministry of Health or allied neonatal care groups and the NICU where this research took place was not required to collect data on KMC. In addition, there was no evidence of quality improvement tools to measure and evaluate KMC programmes within the New Zealand context, although it is feasible that tools from other countries could be adapted for this purpose (AtunEiny \& Scher, 2008; Bergh et al., 2005; Bergh et al., 2012a; Bergh et al., 2014; Skene, Gerrish, Price, Pilling, \& Bayliss, 2015).

Over the last forty years, survivability of extremely preterm infants has improved dramatically, largely because of intensive medical intervention. As knowledge has developed about the psychosocial and neurodevelopmental effects of prematurity and intensive care hospitalisation, a 'perfect storm' of traumatic experience has also been identified (Coughlin, 2017; D'Agata, Young, Cong, Grasso, \& McGrath, 2016; Erdei \& Dammann, 2014; Sanders \& Hall, 2018). Healthcare organisations are being encouraged to make a paradigm shift away from siloed, medically privileged and 
physician-centred frameworks to a model of health care based on patient-centred care, collaborative teamwork, justice and humanism (Behruzi, Hatem, Goulet, \& Fraser, 2014; Charpak \& Ruiz, 2016; da Silva, da Silva, \& Christoffel, 2009; Meleis, 2016). Rich countries are being asked to create novel healthcare solutions based on 'reverse innovations' and lessons learned from poor countries (Syed, Dadwal, \& Martin, 2013), with specific reference to KMC (Syed et al., 2012). In addition, evidence of expensive market-driven overuse of biotechnology for infant care, including rising rates of non-essential NICU admission, is growing (Abadia-Barrero, 2018; Carroll, 2015; Harrison \& Goodman, 2015).

The science of implementation challenges us to "take proven interventions and implement them in the real world" (Peters, Tran, \& Adam, 2013, p. 5). Specific to the field of neonatology, stakeholders are being asked to focus on neuroprotective measures which will improve prematurity-related health outcomes, amidst reducing healthcare budgets (American Academy of Pediatrics, 2012a, 2012b). One such evidence-based, cost-effective strategy is KMC (Nyqvist, 2016, p. 342):

Given these benefits, why has the uptake of KMC as a routine practice been so slow?... follow-up studies of KMC training programmes have found staff indifference and even resistance... perception that it is an inferior poor man's alternative and that it is associated with increased safety risks... So what facilitates the implementation and sustainability of KMC?

Based on these perspectives, I highlight the existence of two conspicuous knowledge gaps, which this research seeks to contribute to. Firstly, very little is known about the characteristics of KMC practice within the tertiary NICU environment in New Zealand; and secondly, evidence about which quality improvement methods are useful for the development of KMC programmes within the New Zealand NICU context is unknown.

\subsection{PURPOSE OF THIS STUDY}

Aim: To use PAR methodology to work in collaboration with NICU community members to explore and improve the quality of Kangaroo Mother Care within their own setting.

\section{Objectives:}

i. to engage with the NICU community about their experience and understanding of Kangaroo Mother Care within their own context; 
ii. to engage multiple stakeholders of the NICU in the co-design and implementation of a KMC quality improvement research project;

iii. to collaborate with key stakeholders in the NICU for the completion of up to three PAR cycles which include exploration, implementation and evaluation of their KMC programme;

iv. offer a facilitation role in quality improvement of KMC by supporting any activities in the NICU relating to their own KMC programme;

v. to be responsive and open to possibilities for research projects that might be put in place around them; and to

vi. present findings back to the community on the suitability and adaptability of PAR methodology within the NICU context for development of KMC.

Over the course of the field work of this study, it became apparent that the initial aim and objectives involving the application of a 'classical' PAR methodological approach, at least in part, were not going to be fulfilled. A combination of factors contributed to a protracted exploratory phase of participatory research that resulted in the inability to complete three iterative study cycles. Despite this, PAR methodology was maintained with acceptable levels of participation with the community for a single exploratory cycle of research. Application of PAR in the real-world complexity of the NICU where this study was conducted required fleetfooted researcher flexibility and multiple adaptations to the inital study design, the approach to which is detailed next.

\subsection{RESEARCH APPROACH}

A PAR approach was chosen to fulfil the primary aim of the study: to work in collaboration with the NICU community to explore and improve the quality of Kangaroo Mother Care within their own setting. Methodological principles of partnership, knowledge-sharing and raising critical consciousness for the purpose of action-based change were proposed as the 'tools' for improvement of the evidencebased practice of KMC. The progression of the research was, at times, hampered by reduced levels of participation and cycles of engagement, non-engagement, and then re-engagement. The combination of time-intensiveness, variable and declining participation, and complex research ethics processes resulted in necessary modifications to the research approach. Adherence to PAR principles ultimately 
enabled the completion of an exploratory cycle of KMC within this unit, the first step of action-based quality improvement.

Two additional theoretical lenses became more prominent over the course of the study, namely critical feminist and Foucauldian theories. The first I was aware of prior to the start of the research, the second was adopted around half way through the project with the inclusion of a discourse analysis. A critical feminist lens had commonalities with both PAR and my personal epistemological and ontological frameworks, and therefore seemed important to foreground. Most importantly to me were non-exploitive, relationship-based research values which engendered increasing social justice, ensuring all participants voices were represented and 'heard' (Jenkins, 2015). I wished to include the mandate of all feminist research to "foster social transformation and social justice for all people" (Anderson, 2000, p. 221) through identification of gender-based power differentials, knowledge democratisation with vulnerable people, in this case, hospitalised women and infants (Anderson, 2000; Jenkins, 2015). I also shared the view of other feminist researchers that "hospitals are still based on a patriarchal system with a strongly held ideology that views professionally based knowledge as superior to patients' experitise (Gristi et al., 2016, p. 239).

The inclusion of a Foucauldian-informed discourse analysis after the completion of the majority of my fieldwork and data analysis was in response to high ambiguity around 'what was being said versus what was being done' with regards to KMC practice. The importance of identifying power relations, discourses and structures affecting KMC within the 'operating system' of the NICU, became increasingly necessary as described by many critical social theorists (Altman, KantrowitzGordon, \& Vandermause, 2014; Barrere, 2007; Griscti, Aston, Warner, MartinMisener, \& McLeod, 2017; Landzelius, 2006; Springer \& Clinton, 2015). Foucauldian concepts informed a methodological process for identifying prevailing discourses within the socially constructed community of the NICU. Addition of findings relating to narratives from women and parents with lived experience of $\mathrm{KMC}$ within the NICU was also gleaned from local and global literature, as well as my own 'NICU-story'. The addition of NICU narratives from outside of my primary research arose because I was only able to secure one parental participant for this study. My concern was that lack of maternal/parental voice, particularly that of 
indigenous Māori, would further marginalise the perspectives of people whom the research most concerns. The effect has been to increase the participation of women and parents with experiences in the NICU, despite their stories not emerging from this primary study.

Whilst the original design of this study planned for the use of a three-cycle particpatory action research approach, between half way and two thirds of the way through the research, a departure from the original design process was required. There were two major factors involved with this adjustment and the commitment to continue with PAR methodology. The first factor involved a critique of whether there were satisfactory levels of participation by NICU community members to justify the research methodology as PAR (table 2). Secondly, the scope of PAR methodology allowed for the adoption of a wide range of methods which acted to strengthen the inquiry, leading to my decision to use a secondary discourse analysis in support of the primary research aim. In addition, findings from within the established literature of whānau Māori perspectives, worldwide parental experiences, as well as my own NICU-narrative were included. In this way, parental voices were combined with findings from two PAR-participant narratives from the primary research in Chapter 5 to strengthen the contribution of NICU consumers. With this decision made, the adjustments allowed field work to continue to a satisfactory end, aligned to the principles of PAR.

\subsection{POSITIONALITY: 'I', THE RESEARCHER}

For the researcher who chooses PAR, there is a particular tension which must be acknowledged and reflected upon for the duration of the study; and that is the one of 'researcher as participant and facilitator'. I understood that inherent in my postition as a co-researcher, was the responsibility to disclose (Probst, 2016). I was to bring my biases to the research group through transparency, full disclosure and ongoing reflexivity, these characteristics lending themselves to relationship building, collaboration and the possibility of emerging knowledge (Ferreyra, 2006). In addition, raising awareness about my own subjectivities - one version of 'truth' would form the basis of a co-creative process with other research participants, creating an environment for reflection, emancipation and a willingness to innovate and activate social change (Ferreyra, 2006). On this note, I make explicit my position as a researcher for this study, including what I consider to be my validity and 
authority to "represent the researched" (Jenkins, 2015, p. 17). I have intentionally used a biographical style to impress upon the reader the epistemological requirement of PAR which holds space for 'researcher as I'.

Embarking on this primary research, I understood that my own worldview was important to consider when choosing a methodological framework. I possess a feministsocial justice worldview, which is woman-centred and focuses on emancipation. In particular, I seek to subvert and be free from predominating powerbased structures and ideologies, which I have often experienced as patriarchal and oppressive. It is within healthcare institutions, particularly during my own maternity care, where I have experienced the discursive effects of power constructs through behaviour I have viewed as coercive and, at times, demeaning. In my view, organisations are hierarchically structured and poorly equipped to engage in meaningful partnership, resulting in the absence or unavailability of relationshipbased decision-making and true informed consent.

Whilst articulating my worldview, I also sense the importance of acknowledging my position of privilege. The opportunity to research and produce a thesis at $\mathrm{PhD}$ level, supported by academic scholarship and other structures afforded to a relatively select few, proves as influential as the multiple other factors which corroborate to produce my subjective truth(s). Having critically reflected on the dominant lens through which I perceive and relate to the world, my simultaneous commitment to remaining open to multiple truths and 'real-time' reflexive learning processes combine to produce my affinity for PAR methodology.

It is my enduring belief that I was well placed to represent the voices of the participant members of this NICU for three main reasons, despite the possibility of being perceived and treated as a 'critical outsider' within this environment (Jenkins, 2015). Firstly, I experienced seven months of NICU services as a 'NICU-mother' of two extremely preterm infants; secondly, I practiced as a clinician (veterinarian) within a biomedical paradigm; and thirdly, I had researched perinatal physiology and $\mathrm{KMC}$ at postgraduate level, which included a research project completed within the NICU environment. The possession of these multiple 'knowledges' provided me the benefit of a shared language with a broad cross-section of NICU community members. 
Next, I discuss two of the major theoretical frameworks which underpin this study, placing my research firmly within the field of maternal-infant healthcare. Brief introductions of these constructs begin with a discussion of developmental biology of the infant, mother and dyad. This is followed by review of developmental care theory for the hospitalised infant including the situation of Kangaroo Mother Care within that theoretical framework.

\subsection{THEORETICAL FRAMEWORKS}

As described, two major theoretical frameworks underpin this work, firstly, developmental biology of the infant, mother and dyad; and secondly, developmental care theory of the hospitalised infant. Infant developmental theory informs us about why KMC is so important for this group of vulnerable babies and caregivers. Developmental care theory informs how caregivers can best meet the needs of hospitalised dyads in the developmentally sensitive postnatal and infancy periods. Brief introductions to these theories are provided in the next sections and are the topics for literature review in chapter two.

\subsubsection{Developmental biology of the infant, mother and dyad}

As the baby transitions from the uterine environment, the biological expectation for the mother-infant dyad is "zero separation" (Bergman, 2014, p. 1), constituted by maternal-infant proximity, uninterrupted skin-to-skin contact and self-attachment to the breast (Brockman, 2015; Feldman \& Eidelman, 2007; Phillips, 2013). It is primarily the somatosensory input provided by mothers' smell and touch which activates hormonal and neural processes within the infant involved with physiological stabilisation, calming, pre-digestion and feeding behaviours (Buckley, 2015; Phillips, 2013; Widstrom et al., 2011). Importantly, the neural constructs of mothering are activated within this context of somatosensory stimulation for the woman (Feldman, 2015a; Flacking, Thomson, \& Axelin, 2016). Within this dyad, optimal sensory experience results in a combination of down-regulation of fearful states (otherwise perceived by the baby as threats), and an upregulation of positive emotional states such as joy and excitement (Schore \& McIntosh, 2011; Swain et al., 2014).

Through physiological and psychosocial lenses, the experience of maternal separation, as with other mammals, is potentially the most potent stressor a baby 
could experience. The result is dominant sympathetic nervous system control which is mediated by cortisol (Aguggia, Suarez, \& Rivarola, 2013; Bergman, 2014; Bergman, 2015; Feeley, Genest, Niela-Vilen, Charbonneau, \& Axelin, 2016; Phillips, 2013). Through a developmental lens, the hospital environment represents a large departure from the optimal postnatal environment and is likely to be a stimulus for toxic neurological stress. The metaphor of 'the perfect storm' has been used by other scholars of preterm birth to refer to the constellation of parental separation, chaotic caregiving and unmitigated pain and stress endured by the vast majority of hospitalised babies (Church, Luther, \& Asztalos, 2012; Erdei \& Dammann, 2014). This 'perfect storm' leads to derangement in physiological and neurological responses to the environmental stressors with serious developmental implications (Ash \& Williams, 2016; Marcellus \& Cross, 2016; Sanders \& Hall, 2018).The importance of the critical examination of care practices and environments which effect, alter or diminish the ability of mother and infant to be in skin-to-skin contact throughout the early postnatal period cannot be understated. For the purposes of this research, the environment and the care methods applied with this group in the NICU are of primary concern, most notably those of developmental care and KMC.

\subsubsection{Developmental care of the infant in the NICU}

Healthcare practitioners and providers are encouraged to reflect on established paradigms and practices which influence the optimal habitat of a newborn baby and their primary caregiver in the postnatal period. Where some care practices are clearly at odds with reproductive biology, such as in the case of separation of mother and baby, scholars warn of the gravity of the effects (Narvaez, Panksepp, Schore, \& Gleason, 2013, p. 6):

In modern societies, the ancient practices that presumably sustained an implicit understanding of the needs of 'the mammalian' brain-mind have been supplanted by 'advanced' cultural practices that may be losing touch with our ancestral needs.

The principles of developmental care inform recommendations for the support of each individual's current strengths, vulnerabilities and thresholds to disorganisation through the optimisation of the external sensory experience being delivered (Als, 1998). Most recently, the experience of the hospitalised infant has been conceptually framed as 'Infant Medical Trauma in the NICU' (IMTN). This model combines knowledge from developmental care theory, neurobiology and human development 
to situate NICU hospitalisation as an adverse childhood experience from the perspective of the patient (D'Agata et al., 2017). The implementation of environmental and care practices which promote optimal development of infant and maternal physiological, somatosensory and neural functioning are expected to positively impact later cognition, behaviour and development (Maitre et al., 2017). Family-centred care (FCC) encompasses all developmental care frameworks and has most recently become the "gold standard in healthcare... a fundamental principle in the provision of neonatal care" (Himuro, Miyagishima, Kozuka, H., \& Mori, 2015, p. 284).

Developmental care frameworks aim to improve health outcomes for hospitalised preterm infants and their families through environmental and care practice modifications which enhance neuroprotection of the baby (Altimier \& Phillips, 2016; Altimier \& Phillips, 2013). Various biopsychosocial frameworks underpin developmental care, with ecological theories that observe immediate and broader environmental influences on the development of the baby and whānau (Kazak, 2006, p. 381):

Pediatric health care is a natural fit with the biopsychosocial model. Children and their families are embedded in complex multiple systems, from cellular through societal. These multiple contexts become differentially activated when a child has a serious and/or chronic health problem. Indeed, the normal course of development for children and families is potentially disrupted when a child becomes ill or is injured, with reciprocal influences across biopsychosocial realms.

More recently, developmental care theories acknowledge parents as the primary nurturers of their child, recognising the importance of the social interconnectedness of infants and their caregivers for healthy neurodevelopment (Als et al., 2011; Altimier, Kenner, \& Damus, 2015; Hall et al., 2017; Sanders \& Hall, 2018). In addition to biological and psychosocial perspectives in healthcare, scholars theorising about holistic obstetric practice have advocated for a paradigm which includes spiritual principles (Davis-Floyd, 2001, pp. S16-S17):

The holistic paradigm also insists on the participation of the spirit in the human whole. In incorporating soul into the healing process, holistic healers bring medicine back into the world of the spiritual and the metaphysical from which it separated during the Industrial Revolution... indeed, chaos theory and systems theory both inform and underpin the holistic paradigm and its insistence on the oneness of body, mind and spirit. 
Considering indigeneity in Aotearoa New Zealand, upholding the legislated requirements of Te Tiriti of Waitangi (The Treaty of Waitangi) and Aotearoa's obligation to affirm Māori as tāngata whenua (people of the land) is crucial to the wellbeing of Māori. (Kenney, 2001). In healthcare, frameworks relating to the holistic wellbeing of Māori women and whānau have been described and constitute an important part of cultural safety and competence, "understanding spirituality is critical to comprehensive and culturally competent healthcare" (Lambie et al., 2015, p. 54). Indigenous principles such as wairuatanga (spirituality), whakapapa (genealogy), karakia (prayer) and whānaungatanga (kinship) are known to be an important part of healthcare (Farry 2014, Lambie et al., 2015).

In 'ordinary life' events, such as the transition to motherhood, scholars Athan and Miller (2013) described a dearth of exploration of spirituality around the topic (p. 221):

The transition to motherhood, a ubiquitous form of quantum change, has not been explored in depth within a spiritual framework despite being universally described as a significant achievement of adulthood for a woman marked by acute redefinitions of self, reappraisals of lifestyle choices, and dramatic shifts in ways of thinking about intimacy, love, relationships, the world, and God.

Whilst there is emerging evidence confirming the spiritual meaningfulness of childbirth, spirituality in healthcare "continues to be on the peripheral of [midwifery] practice" (Crowther and Hall, 2015, p. 173). For women, caregivers and whānau whose babies are hospitalised in the NICU, the omission of explicit attention to their spirituality within the cultural milieu of the nursery may increase the difficulty of an already traumatic event (Baum, Weldberg, Osher \& Kohelet, 2012; Viera, Farias, Santos, Davim \& da Silva, 2015). The combination of societal, organisational and individual influences converge to produce the 'culture' or 'ecology' of a social system which has contributed to multiple barriers to developmental care and KMC on a global scale. Highly contextual factors attributable to health systems, practitioner, and parent/patient perspectives are consistently preventing effective programme development and the breakdown of existing KMC programmes (AbadiaBarrero, 2018; Chan, Bergelson, Smith, Skotnes, \& Wall, 2017; Chan et al., 2016; Griscti et al., 2017; Smith et al., 2017; Soni et al., 2016). The ability to assess and quantify care paradigms as they relate to the 'developmental ecology' within individual neonatal intensive care units, in support of more positive developmental 
outcomes for preterm patients and their families, has therefore become a pressing issue in maternal-infant healthcare (Atun-Einy \& Scher, 2008, White, 2014).

Through skin-to-skin contact using KMC, the biopsychosocial principles of developmental care may be established within the biomedical paradigm of the NICU healthcare model (Chan et al., 2017; Guenther et al., 2017; Hall et al, 2017; Hubbard \& Gattman, 2017; Vesel et al., 2015; World Health Organization, 2015). One neonatologist advocated for the development of care of hospitalised neonates by stating (White, 2014, p. 174):

... there is a bigger picture that should be beyond debate: newborns cannot be fully understood if we only think of them as isolated biological organisms. For too long, we have acknowledged in only the most superficial way that they are affected by their environment and are dependent on their family for biopsychosocial support that our medical care cannot duplicate... kangaroo care is an example of a paradigm shift in an earlier phase of evolution... Thus, the paradigm shift is not yet complete, but the day may be approaching when we will see the incubator not as ideal for safety and cleanliness but rather as a place of sensory isolation and a potential reservoir for nosocomial pathogens, whereas the mother's arms will be recognized as the source of rich sensory input, homeostasis and desirable probiotic organisms.

Kangaroo Mother Care is therefore situated for the purpose of this study as an essential method of caring for hospitalised infants within any developmental care framework, on the basis of human developmental and ecological theory: "Skin-toskin contact (SSC) is the optimal environment for any newborn, but particularly for the premature infant in the NICU" (Altimier \& Phillips, 2016, p. 232).

In summary, two theoretical frameworks underpin the 'why' and 'how' of developmentally appropriate care for hospitalised infants. These inform the premise that the most important aspect of care for the dyad, once medical support for survival has been implemented, is optimisation of the biological expectancies during the postnatal period. Firstly, knowledge of the developmental biology of the infant, mother and dyad inform NICU community members about why it is important to fulfil their biological requirements in the postnatal and infancy periods. Without this knowledge to frame the ways in which we attend and attune to these individuals, our care is akin to bobbing around the ocean without a rudder with respect to how to environmentally support their physiology and development. The second theoretical framework of developmental care, encompassing Kangaroo Mother Care, then provides knowledge informing which strategies, when implemented, will enhance the biological processes of the pair. These processes, that have been genetically 
programmed and 'hard-wired' into the maternal-infant brain, are extremely vulnerable to the effects of the environment within the postnatal period. Care that is provided in absence of these theoretical knowledge bases is likely to be, at best, suboptimal, and at worst, harmful.

\subsection{THESIS OVERVIEW AND ORGANISATION}

The remainder of this thesis describes the exploration, findings, discussion and recommendations relating to participatory research of a Kangaroo Mother Care programme in the context of one NICU. The study employed participatory action research methodology and lenses of Foucauldian and feminist theories to provide a holistic view of KMC in relationship to the experience of babies, parents/whānau/family, staff and other members of a NICU community. To acknowledge the complexity of the study and in an effort to enhance clarity and understanding for the reader, figure 1 represents the timeline, methods and analyses involved with each of the five phases of the study. In Section B, chapters 4 and 5, this graphic is then separated into its five respective phases to head the methods and findings discussion of each phase.

Figure 1: Timeline and Overview of the Study

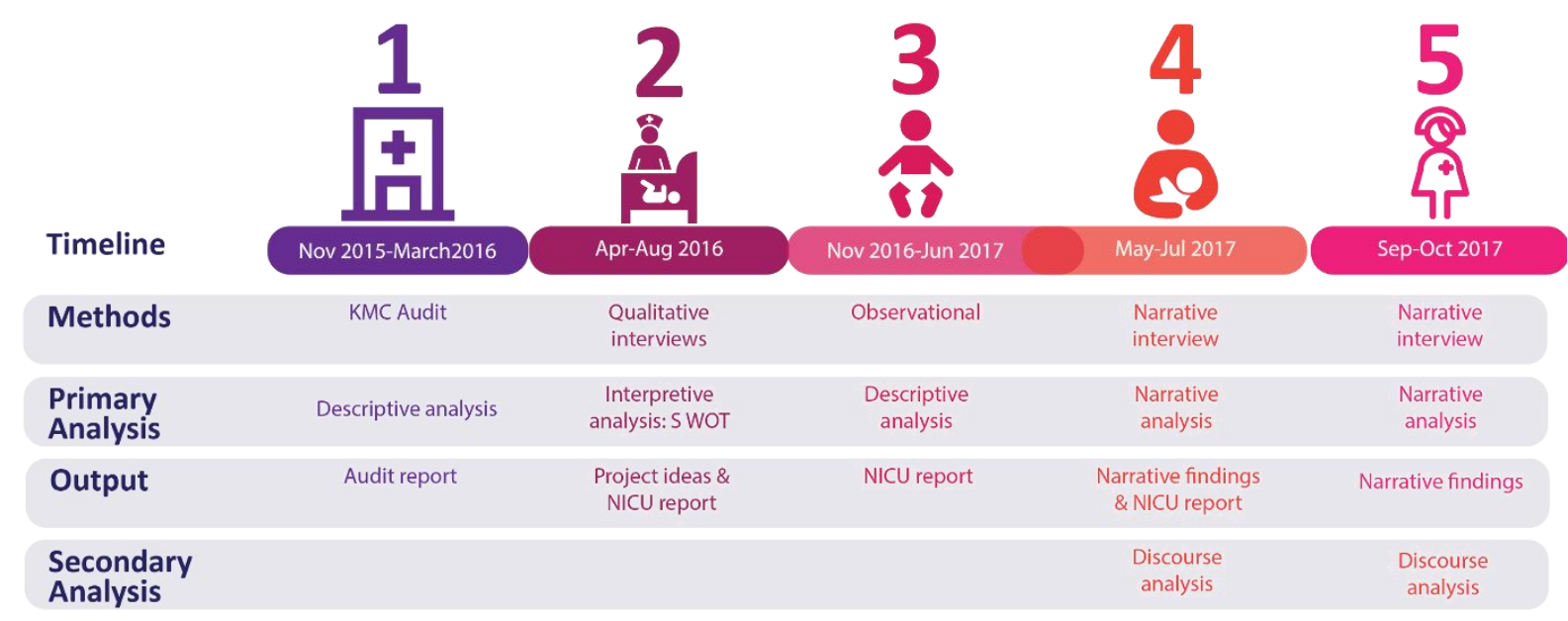

Key:1 = organisational; 2 = staff; 3 = infant observation; 4 = parent participant; 5 = staff participant 
To avoid the burden of overly-long chapters, the thesis is divided into three sections, each with 2 to 3 chapters. Section A contains chapters one, two and three, beginning with the introductory chapter that foregrounds my research interests, the topic and a description of the social construction of NICU-based care in Aotearoa New Zealand. Next, the purpose of the study, including the primary research aim and objectives, was described alongside the rationale for adoption of Foucauldian and feminist lenses for the inquiry. This was followed by my own positioning as PAR facilitatorresearcher. The focus on the two major theoretical concepts that underpin the research came next, those of: developmental biology of the infant, mother and dyad; and developmental care theory for the hospitalised infant, with a specific focus on KMC.

The introduction of these theories set the stage for the literature reviews in section A, chapter two, that look specifically at what is known in four main areas: infant developmental biology; developmental care and Kangaroo Mother Care in the hospital environment; developmental care implementation strategies in the NICU; and the social construction of neonatal care. The review of infant developmental biology is undertaken through the lenses of attachment theory, biological embedding of adverse childhood experiences and toxic stress theories. Next, developmental care literature is examined with respect to current thinking of how hospitalised infant care is best utilised to provide neuroprotection from medical trauma. Thirdly, a review of what is known from implementation science about methodology and strategies for increasing application of evidence-based theory to practice, is described. The last reviews consider literature on the social construction of neonatal care and the neonatal unit, including a focus on institutional racism as a determinant of poor health outcomes for Māori (indigenous peoples) in Aotearoa New Zealand.

The Participatory Action Research approach is thoroughly described in section A, chapter three. Philosophical perspectives about the methodology, the rationale for its use in this case, and the process of its adaptation for the purposes of this research are detailed. Commentary on the levels of participation across the study are then integrated into a discussion on the trustworthiness of the research findings. This is then followed by description of the use of feminist and Foucauldian theories to strengthen the inquiry. Finally, the crucial importance of gatekeepers and champions are discussed. 
Section B contains chapters four and five, both detailing the PAR processes, methods and findings involved with all five phases of field work. Perhaps atypically, the findings were deliberately not put into a separate chapter, the description of which follow on from the methods and analyses of each project phase. In this way, the written findings replicate the process of the fieldwork as the findings from each phase informed the next. Chapter four begins with the preliminary planning phase of the project, including the principles of Te Tiriti o Waitangi as it relates to this research. General ethical considerations and participant engagement are then considered. Chapter four also details each of the first three research phases, comprised of KMC audit, staff interviews, and an observational study. Details of the research rationale, data collection methods, participant recruitment, ethical processes and the flow of the research are described separately for each phase.

Section B, chapter five, consists of two interwoven strands that represent perspectives from people with lived experience of KMC within the NICU. Methods and findings from phases four and five of the primary research project, where one parent and the final PAR-participant were interviewed, describe the conclusion of the first exploratory cycle of field work. The importance of ongoing participation and collaboration with one major stakeholder, the final PAR participant, is foregrounded. Then, in response to low parental participation with my study, findings from analyses of parental narratives from the local and global literature are included, along with understandings gleaned from my own NICU mothering experience.

Section $\mathrm{C}$ holds the final two chapters of the thesis. Chapter six details the rationale, methods and findings from a secondary discourse analysis of data relating to KMC within this NICU's context. It describes inquiry into the previously unexamined power relations affecting the KMC programme, viewed through a Foucauldian lens, using the concepts of power/knowledge and discourse. Discursive findings are explored, combined with theorising about how power relations within the NICU influence KMC practice at individual and organisational levels. The use of discourse analysis as a method within the PAR framework is also discussed.

Chapter 7, the final in Section $\mathrm{C}$ and of this thesis, holds the discussion. It brings together key KMC findings from chapters 4, 5 and 6, and in doing so, tells a KMC story from multiple stakeholder perspectives on this unit (including my own). These 
are discussed in relation to findings from local and global literature relating to maternal, parental and whānau/family perspectives. In addition, methodological findings are described. These include the novel addition of discourse analysis for inquiry into influential power relations affecting KMC, as well as the use of a PAR approach within the complex social environment of the NICU. The most notable contributions to KMC knowledge are described, and the recommendations that are both specific to this location and relevant to other hospital settings. Ultimately, I make a case for the trustworthiness of this work as a legitimate addition of new knowledge to the field of neonatal developmental care, through the exploration of $\mathrm{KMC}$ in the tertiary setting using a PAR approach. 


\section{SECTION A}

\section{CHAPTER TWO: LITERATURE REVIEW}

This study was informed by evidence from two established fields of knowledge, those of human developmental biology and infant developmental care theories. Literature relating to the implementation of Kangaroo Mother Care within the hospitals of high-income countries is also of interest due to the highly contextual nature of KMC practice within different settings. In addition, from a contructivist perspective, the social construction of neonatal care and the patients and whānau whom require NICU services, is important to understand because of its impact on the people's lives who experience it. Literature reviews that synthesise and critique the available research in these areas were conducted.

The first review focused on the current evidence base for infant developmental biology, particularly aspects of neurodevelopment and maternal-infant attachment. The support of optimal biological development of babies within the maternal-infant dyad is particularly important to this study because the biological expectation of all mammals is to remain in constant physical contact with their mother. The hospital environment is likely to result in deviation from the ideal postnatal environment because babies are at higher risk of being separated from their mothers. Maternalinfant separation is a known cause of toxic stress within the neurodevelopmentally sensitive postnatal and infancy periods, causing short and long term negative effects on the brain. Developmental theory informs us of what the biological expectations of the infant and maternal brain are, and the known effects of not meeting those needs. The literature which relates to concepts of attachment theory, biological embedding and toxic stress, which are integral to the understanding of infant developmental biology, are presenced in the review. With current infant developmental biology knowledge understood, frameworks for delivering optimal care to babies and mothers in hospital for the purpose of meeting their biological needs can be constructed and applied.

The next literature critiqued relates to developmental care and KMC theory for the hospitalised infant. It was important to review the current developmental care 
knowledge base to inform research participants about evidence and gaps in this domain for the purpose of relating it to their own setting. This knowledge, in turn, would assist in steering project planning for the groups' own evidence-based change of KMC. Salient concepts within developmental care and KMC theory most relevant to hospitalised infants and this research include: Als' synactive theory; D'Agatas' Infant Medical Trauma in the NICU (IMTN); Adverse Childhood Experiences (ACE); and trauma-informed neuroprotective care.

Thirdly, literature associated with the implementation of KMC within the NICUs of high income countries was examined to ascertain which, if any, studies have been conducted in contexts similar to the one with which I was collaborating. This review was highly relevant to the study because of the known complexities of quality improvement practice within this environment. Its purpose was to identify which methodologies held a precedent for the research of KMC within high-income neonatal intensive care units, in addition to the possibility of addressing specific gaps in $\mathrm{KMC}$ quality improvement science within this knowledge space.

Lastly, literature associated with the social construction of neonatal intensive care, the NICU setting, and the community it involves, was important to examine through the lens of constructivism. The participatory action approach to this research assumes that the social world of the NICU is constructed by people whose experiences (and the meaning they attribute to them) are the focus of inquiry. In addition, social reality is constructed through multiple discourses that develop meanings for groups living and negotiating a shared experience (Williamson, 2006). Through a constructivist lens, what constitutes knowledge within the NICU 'space' is likely to be dominated by the biomedical paradigm, the influences of which undoubtedly affect the experience of babies, their whānau and the NICU-staff (Carnevale, 1998). The fourth literature review sought to examine what is currently known about the sociocultural concepts framing knowledge within the NICU, and in particular, what is considered 'fact' and "who determines how we know what we know?" (Carnevale, 1998, p. 510). In addition, knowledge relating specifically to the NICU-contexts within Aotearoa New Zealand were explored, most notably, institutional racism. 
Four literature searches were conducted over the course of the study using various databases well known to the nursing, midwifery and allied health sectors, including: Pubmed; CINAHL Complete; ProQuest Nursing and Allied Health; Te Waharoa Victoria University of Wellington; and finally, the Cochrane Library. Searches were initially conducted globally and subsequently more locally (New Zealand/Australia) based on the assumption that the nature of healthcare implementation is highly contextual and would not necessarily be able to be generalised to other settings. The outcomes of the searches were markedly different with respect to the scope of literature available. Grey literature sources were also explored, where appropriate, and I used the references of any recent systematic reviews on the topics to crossreference the initial database searches.

\subsection{REVIEW ONE: INFANT DEVELOPMENTAL BIOLOGY}

Infant developmental biology and maternal-infant attachment have occupied a space of inquiry within the life sciences since the early 20th century. Accordingly, scholarship on this topic was so extensive, in the tens of thousands of articles, as to require a process for filtering the vast amounts of information. To this end, I focused on the most current and condensed empirical knowledge presented within two publication types - literature and systematic reviews - assuming that the body of knowledge from theoretical and research studies would be captured by this broad approach. The following search terms were applied after the initial broad scope of infant developmental biology search results to aid in the identification of the most relevant papers:

i. Articles published between 2000 and 2016;

ii. Literature and systematic reviews only;

iii. Articles written in English;

iv. Articles from peer-reviewed journals;

v. Articles which included the search terms within the abstract, main body or reference sections;

vi. Articles published worldwide and then; 
vii. Articles based on research in New Zealand and Australia (for the second search).

Current accepted knowledge of developmental biology of the human infant has arrived through intersection of multiple fields of scholarship such as developmental psychology, evolutionary biology, sociology and neuroscience (Cicchetti \& Toth, 2009). Research inquiries using both animal and human studies, with qualitative and quantitative methodologies have been used to investigate the human infant life stage and as already noted, are prolific within the established literature. Theory and findings pertinent to a developmentally 'normal' postnatal period are foregrounded, with the aim of contrasting it to a postnatal period which occurs in the hospital environment. Established theory highly relevant to the hospitalised infant, particularly one born preterm include: attachment theory, biological expectation during the postnatal period; biological embedding of early adverse experiences; and toxic stress.

\subsubsection{Attachment theory}

Attachment is defined by parenting scholar Benoit (2004) as "one specific and circumscribed aspect of the relationship between a child and caregiver that is involved with making the child safe, secure and protected" (p. 541). Attachment remains the most established scientific theory relating to the relationship between children and their caregivers, the premise of which continues to evolve from John Bowlby and Mary Ainsworth's work of the first half of the $20^{\text {th }}$ century (Benoit, 2004). Now highly integrated with neurodevelopmental science and psychobiological theory, attachment theory affirms early and enduring physical closeness with their primary caregiver as being "a vital biological function" (Hofer, 2006, p. 84), essential to the physical, emotional and cognitive development of the baby (Kommers et al., 2016; Niela-Vilen, Feeley, \& Axelin, 2017; Vanderbilt \& Gleason, 2011; Zmyj, Witt, Almut, Neumann, \& Lucke, 2017).

Theoretical modelling from animal studies supports the presence of an inborn human drive to seek attuned caregiving and attachment from birth, the quality of which is highly dependent on the infant experience within this period (Liu et al., 2007; Perry, Blair, \& Sullivan, 2017). This genetically programmed signal is expressed as a complex set of behaviours, observable at birth where optimal physiological and 
environmental conditions are favourable (Widstrom et al., 2011). Both mother and baby are linked in this attachment process - reciprocity is at play - with both members of the dyad undergoing neurological transformation in the postnatal period via neurohormonal pathways involving the oxytocin, prolactin and beta-endorphin systems (Aguggia et al., 2013; Buckley, 2015; Feldman, 2015a). Evidence from animal models of early maternal deprivation reports lifelong negative effects on offspring, including alterations in behavioural and endocrine responses to stress (Gunnar \& Quevedo, 2008; Levine, 2005; Meaney \& Szyf, 2005), and is now known to be transmissible to subsequent generations (Maddalena, 2013; Provenzi \& Montirosso, 2015; Samra, McGrath, Wehbe, \& Clapper, 2012). Whilst the mechanisms for the negative developmental effects of poor attachment have not yet been fully elucidated, current theoretical knowledge describes how environmental effects on the epigenes and cellular structures 'programme' affected infants and "gets under the skin" (Thomas et al., 2017, p. 425).

Thus, current accepted theoretical knowledge, supported by experimental animal modelling, is that secure attachment is: scaffolded by neurobiological mechanisms (Hofer, 2006; Perry et al., 2017); vulnerable to the effects of the immediate postnatal environment (Aguggia et al., 2013; Kommers et al., 2016; Weatherston \& Browne, 2016); forms the basis of enduring attachment patterns over the lifetime (BraungartRieker et al., 2014; Perry et al., 2017; Schore \& McIntosh, 2011); and is one factor involved in enduring positive infant mental health (Ashby \& Bromberg, 2016; Lopez-Maestro et al., 2017).

Preterm and hospitalised infants are known to be at higher risk for disrupted attachment compared with full term babies, one mechanism by which these infants are known to have reduced mental health and long term poorer cognition (Ashby \& Bromberg, 2016; Del Fabbro \& Cain, 2016; Pennestri et al., 2015; Weatherston \& Browne, 2016). In their review aimed at raising clinician awareness of the deleterious effects of less-than-ideal bonding in the NICU setting, Kommers and colleagues (2016) state that whilst "the physiological consequences of suboptimal bonding are less frequently addressed in the literature than those of other threatening unnatural stimuli" (p. 738); the evidence is clear that there are (possibly reversible) developmental effects through neurohormonal and epigenetic mechanisms. Neurodevelopment and attachment theory scholars recommend primary prevention 
and/or early intervention for the mitigation of compromised attachment and suboptimal neurobiological effects contributed to by maternal-infant separation in infancy (Kommers et al., 2016; Perry, Blair \& Sullivan, 2017; Neczypor \& Holley, 2017; Vetulani, 2013).

Literature relating to parental and professional perspectives regarding the support of attachment within the hospital, presents findings on who should be involved and how it can be optimised. Firstly, NICU staff and parents acknowledge the importance of good quality attachment for the physical, emotional and cognitive benefits of babies and families (Brockman, 2015; Del Fabbro \& Cain, 2016; Fleck, 2016; Franklin, 2006; Karl \& O'Hare, 2006). Parents and healthcare practitioners generally perceive skin-to-skin holding of their infants as important for the bonding process and a 'notable first time event' in the new lives of their hospitalised babies (Baylis et al., 2014; Chia, Sellick, \& Gan, 2005; Engler et al., 2002; Solomons \& Rosant, 2012). Despite the ideological support for care which supports attachment, large systematic reviews report the multiple barriers which exist at systemic, professional and parental levels to prevent the implementation of skin-to-skin care (Chan et al., 2017; Chan et al., 2016; Seidman et al., 2015; Smith et al., 2017). In the majority of settings, it is the neonatal nurse who is considered to be perfectly placed at the bedside of parents and infants, to facilitate attachment for the maternal-infant dyad, framed by Karl \& O’Hare (2006) as the "nurse attacher" (p. 258). Additional qualitative studies have described parental views about the factors which enhanced their feelings of resilience, capacity to care for, and begin to attach with their infants (Aagaard \& Hall, 2008; Adkins \& Doheny, 2017; Rossman, Greene, Dratovil, \& Meier, 2017). Authors of a meta-synthesis of 14 qualitative studies of NICUmothers' experiences describe key themes centred on the reciprocal relationship of mother and baby. Findings included the gradual nature of attachment for this group (Aagaard \& Hall, 2008, p. e31):

The mother might fear attachment to the new baby... [the mother] has a strong desire to be close to and get to know her baby while, at the same time, worrying about her baby's wellbeing... [mothers needed] an opportunity to see, touch, hold, provide skin-to-skin contact, supply breast milk, and care for her infant. 
Literature is also established at the global health system level which advocates for the basic human right of an infant to have their experience of attachment validated valorised (World Association for Infant Mental Health, 2016, p. 4):

The infant therefore has the right to have his/her most important primary caregiver relationships recognized and understood, with the continuity of attachment valued and protected - especially in circumstances of parental separation and loss.

An adjunct to the UN Convention on the Rights of Children, infants basic rights' are underpinned by the principles of developmentally-appropriate care. This framing is designed to guide and support social and health policy based on the premise that the needs of infants and toddlers are arguably different from those of older children due to their extremely dependent state (World Association for Infant Mental Health, 2016). Integration of developmental knowledge with child rights discourse is strengthening within the current healthcare literature, particularly where there is trauma involved (Del Fabbro \& Cain, 2016, pp. 283, 284):

preventive efforts to support and sometimes restore parent-fragile infant attachment results in improved developmental, behavioral, and social emotional outcomes for the infant... can best serve the needs of the families identified at high risk.

There is, therefore, an unambiguous call to action for the implementation of frameworks which advocate for the protection of maternal-infant attachment within the postnatal period in all global settings (American Academy of Pediatrics, 2012b; Ash \& Williams, 2016; Coughlin, 2011; Huebner et al., 2016; World Health Organization: Western Pacific Region, 2014).

\subsubsection{Biological expectation in the postnatal period}

The evidence is clear that the development of attachment and other 'necessary biological processes' are most crucially dependent on the environment and care which is provided within the postnatal period (Bergman, 2015; Narvaez et al., 2013; Porges \& Furman, 2011). What knowledge, then, is present in the literature which provides further detail about the specific biological expectation of the infant, mother and dyad within the postnatal period, particularly with respect to the hospitalised infant?

Empirical theoretical evidence exists for the developmental needs of all mammalian species, with the biological expectancies of the human infant in the postnatal period 
characterised by qualities of (physical and emotional) proximity, sensitivity, attunement and contingent responsiveness and connectedness with their caregivers (Sanders \& Hall, 2018). In the optimal state, ongoing close maternal proximity is provided by uninterrupted skin-to-skin contact, "the normal mammalian postnatal condition" (Goldstein Ferber \& Makhoul, 2004, p. 858). Attachment processes continue and breastfeeding within the first few hours of birth occurs (Bergman \& Bergman, 2013; Feldman, 2007; Neczypor \& Holley, 2017; Phillips, 2013; Robiquet et al., 2016). The result is a social connectedness which signifies to the babies' brain that it is safe (Goldstein Ferber \& Makhoul, 2004; Phillips, 2013; Widstrom et al., 2011). Social connectedness is a "biological imperative for mammals in their quest for survival" (Porges, 2015, p. 116), and is present between an infant-caregiver dyad when mutual regulation of physiological and behavioural states is achieved. This state occurs as a result of optimal somatosensory input from the mothers' smell and touch, underpinned by unconscious activity of the parasympathetic branch of the autonomic nervous system (Buckley, 2015; Feldman et al., 2014; Luong et al., 2016; Phillips, 2013). More than simple nurturing is achieved: attuned reciprocal interactions between baby and caregiver provide necessary neural stimulation which cues safety and promotes physiological and biobehavioural regulation (Feldman, 2015b; Porges, 2015).

The disruption of the mother-infant relationship through physical separation in the postnatal period, therefore, is in direct opposition to human biological expectancy. The high likelihood that the NICU environment antagonises infant and maternal development has been established in the literature in accordance with principles of ecobiodevelopmental theory and the neurodevelopmental effects of environmental chaos (Coley, Lynch, \& Kull, 2015; Coughlin, 2017; Hall et al., 2017; Johnson, 2013; Kim, 2016; Patterson \& Vahili, 2014): “Infancy represents an exceptionally sensitive period of development characterized by vulnerability to environmental insults" (Coley et al., 2015, p. 96). Where hospital-based care is required, environmental and care paradigm modifications which support feelings of safety for the infant and caregiver by meeting their biological relationship-based needs are urgently required (Hall et al., 2017). Empirical evidence for the biological expectations of the dyad, reinforced by developmental theory, unequivocally state the requirement for relationship-based care which provides social 
interconnectedness. For the newborn infant, the optimal habitat is in direct contact with their primary caregiver: "Skin-to-skin contact requires a parent's presence to provide the only place nature intended neurodevelopment to occur" (Bergman, 2015, p. 145).

\subsubsection{Biological embedding of early adverse experience}

So how, exactly, do unfavourable experiences affect the newborn in the long term? The processes by which early life experience alters human development and biology in the long term have emerged within the literature over the last 25 years, now commonly termed 'biological embedding' (Berens et al., 2017; Hertzman, 2012; Morgan, 2013; Thomas et al., 2017). The principle of biological embedding has been described as "when experience gets under the skin and alters human biological and developmental processes... when they have the capacity to influence health, wellbeing, learning or behavior over the life course" (Hertzman, 2012, p. 17160). For the purposes of this research, the NICU is positioned as an environment which is likely to represent an early adverse experience due to ways in which it deviates from the biological expectation of the infant. The NICU environment, therefore, tends to deliver experiences to infants which get under the skin (become biologically embedded), produce trauma and cause 'toxic stress' for the infant (Ash \& Williams, 2016).

Evidence suggests that biological embedding, a developmental process by which an individual can adapt rapidly to the conditions of the environment, occurs mainly through the actions of the stress-response system, the hypothalamic-pituitary-adrenal (HPA) axis (Thomas et al., 2017). Whilst knowledge relating to the HPA-axis system is important for understanding the concept of biological embedding as it relates to the hospitalised infant, the details of the mechanisms are outside the scope of this review and will not be presented here. Suffice to say that whilst physiological and behavioural responses by the stress response system are important for healthy infant neurodevelopment, its frequent or prolonged activation may ultimately result in physical or emotional dysfunction due to maladaption of the mechanisms involved (Tsigos \& Chrousos, 2002).

Based on current empirical knowledge, the long term developmental effects of environmental stressors on the HPA axis of premature and hospitalised babies in the 
postnatal period, so-called gene-environment interactions, are an area of strong concern for neonatologists and maternal-infant healthcare advocates (Fox et al., 2010; Montirosso \& Provenzi, 2015; Provenzi \& Montirosso, 2015; Shonkoff, 2016; Shonkoff, Garner, The committee on Psychosocial aspects of child and family health, Committee on Early Childhood, \& Section on Developmental and Behavioral Pediatrics, 2012). In one recent systematic review, developmental psychiatry scholars conclude (Turecki \& Meaney, 2016, p. 87):

There is a substantial theoretical and empirical research supporting an association between early-life environmental adversity and poor lifetime mental health outcomes. A critical issue concerns the molecular mechanisms that account for such strong and long-lasting effects.

Scientists acknowledge that more studies are required to assess the mechanisms for programming influences of early postnatal environment adversity on the HPA-axis and neurodevelopment (Montirosso \& Provenzi, 2015; Moore, Berger, \& Wilson, 2014; Turecki \& Meaney, 2016). In the meantime, all efforts to reduce and ameliorate known stressors in this period are of the highest priority (Coughlin, 2017; D'Agata et al., 2017; Sanders \& Hall, 2018). Toxic stress in the hospitalised neonate, and the trauma-informed neuroprotective care which aims to reduce it, are newly emerging areas of knowledge from neurodevelopmental literature, the awareness of which is extremely important to NICU communities.

\subsubsection{Toxic stress}

For the purposes of this thesis, 'toxic stress' is defined as: "strong, frequent, and/or prolonged activation of the body's stress-response systems in the absence of the buffering protection of stable adult support" (Shonkoff, 2010, p. 360). The term was first in use in the psychological literature associated with post-traumatic stress disorder of war veterans. Increasingly, the concept has been applied to child development, featuring in published reports by the American Academy of Pediatrics on the lifelong effects of early childhood adversity (Shonkoff, 2010; Shonkoff et al., 2012). The bulk of established literature reports major risk factors for toxic stressinduced outcomes for children in connection with poverty, chronic abuse and/or neglect and parental substance abuse (Shonkoff, 2010). However, it is important to note that, viewed through a neurophysiological lens, any care practices resulting in perceived threat by a child's brain may equally be classified as conditions causing 
toxic stress. This is particularly so if they occur in the absence of an attuned adult and become chronic and enduring.

Through this lens, neurological trauma can be seen to result when the type or timing of care practices are inappropriately matched to the biological needs of the infant or mother (Perry, 2009). The hospitalised preterm baby is effectively an extra-uterine fetus, whose brain, at a time of extraordinarily high neuroplasticity, encounters an environment which is unable to meet its biological expectancies. They are therefore vulnerable to the effects of toxic stress due to three major factors:

i. Reduced/absent maternal (parental) caregiving and disrupted dyad coregulation (Bergman, 2015; Cho et al., 2016; Evans \& Porter, 2009; Feldman, 2007, 2015b; Feldman, 2015c; Luong et al., 2016; Zmyj et al., 2017);

ii. Exposure to prolonged, strong, and frequent painful or noxious procedures, inappropriate noise and light levels (Bastani, Rajai, Farsi, \& Als, 2017; Gao et al., 2015; Grunau, 2013; Holditch-Davis, Scher, Schwartz, \& HudsonBarr, 2004; Montirosso \& Provenzi, 2015);

iii. The timing of these adverse experiences occur during a neurodevelopmentally sensitive period of growth (D'Agata et al., 2017; Feldman, 2004; Feldman, 2015d; Fox et al., 2010; Thomas et al., 2017).

Knowledge about the mechanisms which produce toxic stress has continued to emerge from neurodevelopmental literature. Again, whilst outside the scope of this review, it is the physiological cycles of compensatory activity and recovery which occur in response to a stressor that result in a process of allostasis or: "achieving stability through change" (McEwen, 2007, p. 880). Under chronic or severe environmental stress conditions, such as those experienced by some hospitalised babies and caregivers, stimulation by stressors is more likely to be cumulative and result in maladaptive response patterns and physiological wear and tear, exaggerated inflammation and oxidative stress (Moore, Berger, \& Wilson, 2014). Characteristic effects of allostatic overload and toxic stress include the disruption of organ structure and brain architecture, and alteration of stress management systems, inversely proportional to gestation at birth, and persisting into adulthood with long-term cognitive impairment (Shonkoff, 2010; Moore et al., 2014). 
To conclude, there is strong theoretical evidence, moderately supported by empirical knowledge from animal studies, that the intersection between infant development, genetically programmed expectancies and postnatal environment represent a crucial life stage of the infant, caregiver and dyad. The most profound unmet biological need, as with all mammals, is due to the separation of infants from their primary caregivers and the resulting absence of attuned nurturing care and social connectedness (Altimier, 2015; Coughlin, 2013; D'Agata et al., 2016; Marcellus \& Cross, 2016; Porges, 2015; Sanders \& Hall, 2018). Most recently, emerging theoretical evidence strongly suggests that NICU-hospitalisation constitutes an early adverse childhood event, especially when enduring maternal-infant separation is the predominant standard of care provided (Altimier \& Phillips, 2016; Coughlin, 2017; D'Agata et al., 2017; Provenzi \& Montirosso, 2015; Sanders \& Hall, 2018). Because of this and the unfavourable effects of pain and environmental chaos, toxic neurophysiological stress is a likely result of hospitalisation for babies and their parents, and possibly endured by staff in this environment as well (Ash \& Williams, 2016; Sanders \& Hall, 2018).

The basis for my proposed research was to use current scientific knowledge as rationale and impetus for the improvement of the group's Kangaroo Mother Care programme. Evidence-based practice can be used to transform traditional routines related to birth provider contexts, particularly where these routines increase levels of maternal-infant separation. Given that sub-optimal bonding is a known risk factor for impaired hormonal and neurodevelopmental outcomes, a strong imperative exists to align maternity care practice with the latest knowledge relating to infant and maternal biological expectation (Kommers et al., 2016). A valuable question, therefore, is: which care paradigms, practices and environmental conditions promote the evolving capacity of the infant to attain optimal attachment and in doing so, increase the opportunity for positive long-term neurodevelopmental outcomes? To answer that question, findings from a literature review of NICU developmental models of care and practices which preserve the integrity of the mother-infant bond, in particular $\mathrm{KMC}$, are explored next. 


\subsection{REVIEW TWO: DEVELOPMENTAL CARE AND KMC IN THE NICU}

A search of the literature on developmental care and KMC within the NICU yielded a total of 312 results. After exclusion of duplicate and irrelevant studies, 89 articles of significance to developmental care and KMC within the NICU remained. I categorised the papers as one of five types of publications: reviews (literature, systematic and meta-analyses), 21; quantitative studies and randomised controlled trials (RCTs), 31; qualitative studies, 11; mixed method studies, 3; and peerreviewed expert opinion/commentary and/or guidelines, 23 (figure 1).

The following search limits were applied to aid in the identification of the most current and relevant papers. The global literature was important to capture overarching themes for all geographical settings, as was an examination of literature related specifically to the Australasian setting:

i. Articles published between 2000 and 2016;

ii. Articles written in English;

iii. Articles from peer-reviewed journals;

iv. Articles which included the search terms within the abstract, main body or reference sections;

v. Articles published worldwide;

vi. Articles based on research conducted in New Zealand and Australia. 
Figure 2: Search Strategy Algorithm of Developmental Care and KMC in the $N I C U$

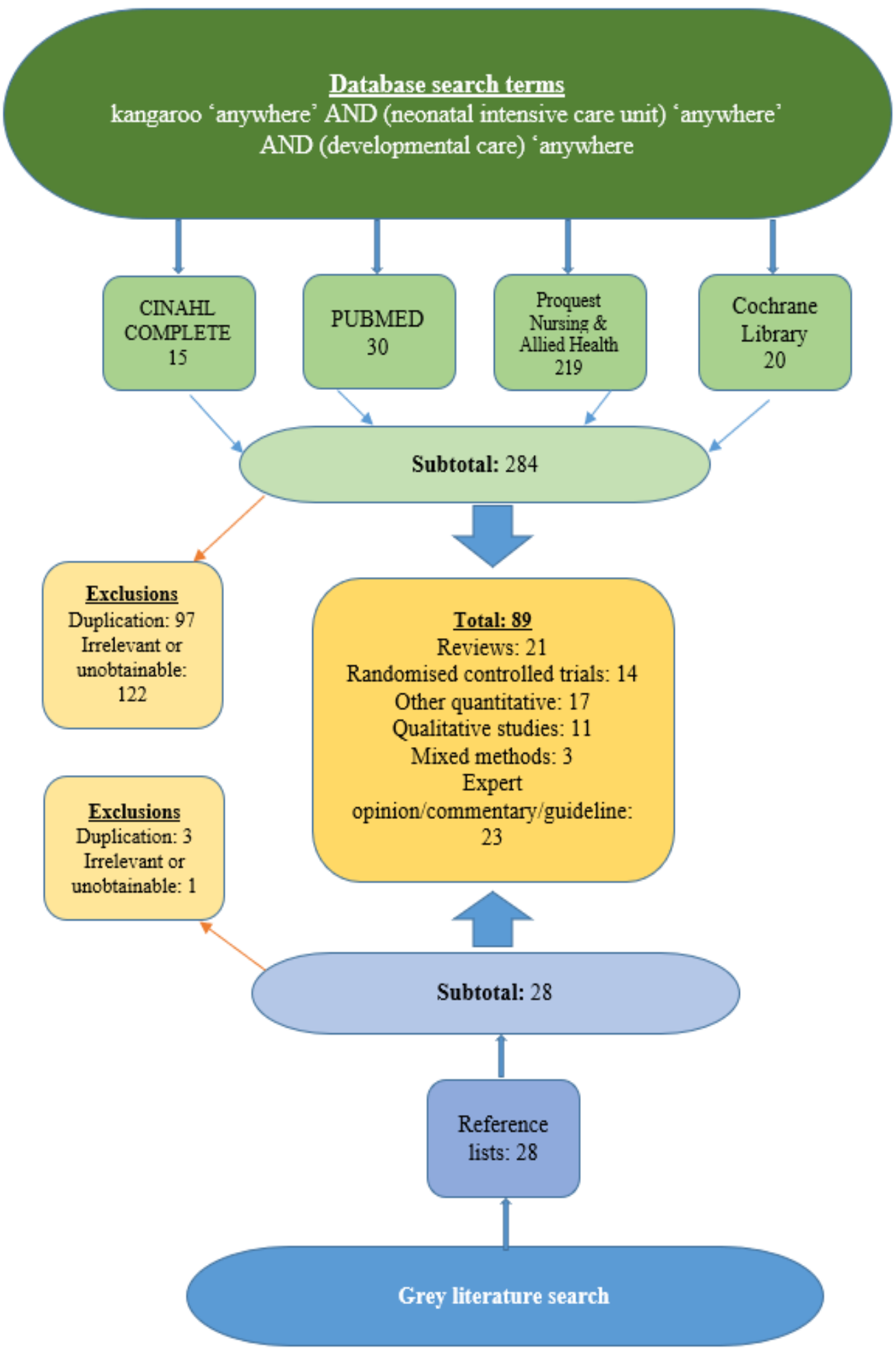


A broad range of theoretical, research and practice-based studies emerged from the search, contributed to by a wide variety of methodologies. Increasing amounts of moderate to high quality literature have recently emerged on the topics of developmental care and KMC in the NICU setting. Almost 60\% (53/89) of the relevant articles published in the last 15 years have emerged in the last 5 years. In contrast to this, with respect to local New Zealand and Australian literature, just six papers have emerged since 2000, only $30 \%$ of which have been in the last 5 years. Findings from multiple scientific disciplines have converged to inform the care of preterm and hospitalised infants, underpinned by theoretical concepts which are highly relevant to this research, including: Als' Synactive Theory; D'Agata's Infant Medical Trauma in the NICU; the Adverse Childhood Experience framework; and trauma-informed neuroprotective care.

\subsubsection{Developmental care theory and practice}

The importance of developmental care as an integrated component of medicalised care for hospitalised babies is highlighted within the literature. In support of enhanced physical, psychosocial and humanitarian outcomes for babies and their whānau/families, evidence-based developmental care practices are considered an essential foundation of neonatal care (Atun-Einy \& Scher, 2008; Fialho, Vargas, \& Santos, 2016; Hendricks-Munoz et al., 2010; Kiechl-Kohlendorfer et al., 2015; Montirosso et al., 2016; Pierrat, Goubet, Peifer, \& Sizun, 2007). Developmental care of hospitalised infants is most strongly informed by Als' Synactive Model of Neonatal Behavioral Organization, a conceptualisation of neurobehavioural development emerging from neurobiological knowledge of the 1970s (Als, 1982). In addition to neurodevelopmental perspectives, viewed through a developmental care lens, infants are considered active participants in their life experience, with family members the most important caregivers in the babies' lives (Als et al., 2011; Altimier \& Phillips, 2013). Als' theory furthered Brazelton's work which recognised the capacity of infants to give behavioural cues which reflect and communicate their own current neurobiological state. (Gibbins, Hoath, Coughlin, Gibbins, \& Franck, 2009).

The central premise of Synactive Theory states that the neurobiological subsystems which are the basis of infant behaviour are inextricably linked to and influenced by environmental and caregiving modalities. Leading on from this premise, it is 
theorised that alterations in the function and structure of the preterm newborn brain may result from developmentally inappropriate caregiving and/or environmental stimulation. Substantial research has been conducted on the efficacy of various developmental care programmes to enhance a broad range of indices of preterm infant and whānau/family wellbeing. Various programmes include, but are by no means limited to: the Neonatal Individualized Developmental Care and Assessment Program (NIDCAP); Family-centred care (FCC), Family Integrated Care (FICare), and Family Nurture Intervention (FNI) (Aloysius, Platonos, Theakstone-Owen, Deierl, \& Banerjee, 2018; Als et al., 2011; Hall et al., 2017; Hendricks-Munoz et al., 2010; Rick, 2006; Thomson, Moran, Axelin, Dykes, \& Flacking, 2013; Welch et al., 2015). Synactive theory informs NIDCAP, perhaps the most recognised developmental care programme in the NICU. The NIDCAP modality works with the central premise that each infant's individual strengths and vulnerabilities are best supported through the preservation of the mother-infant relational dyad and minimisation of the potentially negative environmental impacts of the NICU (Als et al., 2011).

One Cochrane meta-analysis of 36 randomised controlled trials of various developmental care models was inconclusive about the benefits to infants, whilst noting that no harmful effects were reported (Symington \& Pinelli, 2006). Authors of this large Cochrane review found that the heterogeneity of four major developmental care programmes, which included 19 subcategories and multiple interventions, led to difficulty in assessing the effect of any single intervention (Symington \& Pinelli, 2006). Other systematic reviews and meta-analyses in 2013, 2009 and 2002 also found that there was inconclusive evidence to support NIDCAP as a framework of developmental care, although no major harmful effects were noted and more evidence was required (Jacobs, Sokol, \& Ohlsson, 2002; Ohlsson \& Jacobs, 2013; Symington \& Pinelli, 2006; Wallin \& Eriksson, 2009). All three systematic reviews stated that it was methodological shortcomings which prevented far-reaching claims on the "promising findings" of the NIDCAP intervention (Wallin \& Eriksson, 2009, p. 54), and the current body of knowledge would benefit from a comprehensive study which included a clear focus on primary outcomes and extended follow-up. 
In contrast to the findings of aggregated developmental care programmes presented by Syminton and Pinelli (2006), published findings from other multiple systematic reviews, meta-analyses, randomised controlled trials and quasi-experimental studies did show enhanced outcomes related to developmental care. For example, improved developmental outcomes and less disability at 2 years and $5 \frac{1}{2} 2$ years of age (Rick, 2006) and greater quality of life scores at 5 years (Montirosso et al., 2016).

Improvements across multiple infant neurodevelopmental, psychomotor, social and behavioural domains were shown (Kiechl-Kohlendorfer et al., 2015; Welch et al., 2015), as were higher mental and behavioural scores in children at 3 years, although not significant by 5 years (Vanderveen, Bassler, Robertson, \& Kirpalani, 2009). In addition, there were clinically meaningful improvements in some maternal psychosocial indices, such as maternal anxiety and depressive symptoms (Benzies, Magill-Evans, Hayden, \& Ballantyne, 2013).

Findings from my review of the literature reveals that heterogeneity of developmental care interventions, as well as limitations in research design and quality remains a barrier to conclusive findings about the clear benefits of specific programmes (Symington \& Pinelli, 2006; Vanderveen et al., 2009). As well as this, the broad, overarching categorisation of many early interventions and care models in the NICU as 'developmental care' (DC) has proved problematic in attempting to assess developmental care as one homogenous group (Milette, Martel, da Silva, \& McNeil, 2017a, p. 47):

...its [DC] implementation remains challenging, very sporadic, inconsistent, and variable from one setting as well as from one professional to the next, despite the presence of multiple recommendations, experts in the field, and the availability of training programmes.

Further complexity is added by inconsistent implementation of DC across NICUs, highlighted when research has been conducted in multicentre trials (Montirosso et al., 2016). It was noted in one study of developmental practices across Israel, that very little focus had been applied to studies of developmental ecology worldwide, resulting in a paucity of knowledge about which developmental care practices were being applied, to what extent and with what results (Atun-Einy \& Scher, 2008).

My conclusion from a review of the literature is that whilst significant gaps exist in the empirical evidence relating to the efficacy of various modes of DC (mainly due to the lack of standards by which to measure it consistently), there is consensus in 
support of developmental care frameworks as a neuroprotective strategy for preterm neonates. Evidence is sufficient that expert opinion from multiple nursing associations strongly favours the age-appropriate and neuroprotective principles of DC. Moreover, there has been enough evidence that several guidelines recommending best practice of DC have been published over the last decade (Coughlin, 2013; Milette et al., 2017a; Milette, Martel, da Silva, \& McNeil, 2017b; VandenBerg, 2007). Recommendations for parent-infant relationship building and environmental and organisational culture modification are also present (Flacking et al., 2012; McGrath, Cone, \& Samra, 2011; Milgrom et al., 2010; Pineda et al., 2014; Reynolds et al., 2013).

The clear and documented objective of the integration of a care framework which considers both the environment and the developmental biology of the infant is to decrease toxic stress during critical and senstitive periods of development such as the postnatal period (Coley, Lynch, \& Kull, 2015; Milette et al., 2017a; Shonkoff et al., 2012). Of particular note, is the absence of literature relating to the evidence base for the use of conventional incubator care for the physiologically stable preterm infant, either on its own or as compared to skin-to-skin contact (White, 2011). In contrast, safety and enhanced physiological stability for the healthy full- and preterm infant in skin-to-skin, when compared with incubator care, has a moderate and emerging evidence base, represented by randomised controlled trials and systematic reviews (Bergman, Linley, \& Fawcus, 2004; Lorenz et al., 2017; Luong et al., 2016; Moore, Bergman, C., \& Medley, 2016).

With respect to local studies and literature, three reports emerged from the search of the Australasian literature, two of which researched the experiences of Australian mothers within the NICU. The first two articles provided broad recommendations in support of DC practices which enhance attachment and aid women to become competent mothers within the NICU (Evans, Whittingham, \& Boyd, 2012; Fenwick, Barclay, \& Schmied, 2001). The New Zealand paper describes a multi-centred implementation trial of FICare which is yet to be completed, the results of which remain currently unavailable (O'Connor, 2016).

In summary, despite moderate consensus within the literature for developmental care as a safe and 'best practice' for the care of premature infants within the NICU 
environment, further good quality research is required to confirm a consistent positive effect on the outcomes of preterm babies relating to specific DC practices (Atun-Einy \& Scher, 2008; Milette et al., 2017a, 2017b; Philpott-Robinson, Lane, Korostenski, \& Lane, 2017). Quality improvement research involving benchmarking of developmental care strategies across the population and feasibility studies of action plans for its upscaling, are required. A small amount of this research already exists, for example, one Israeli study of developmental practice from which emerged a tool for measuring the various domains of a developmentally appropriate practice (Atun-Einy \& Scher, 2008). Results from this high quality study concur with Als' assertion that despite the shift toward a new developmentally appropriate model of neonatal care and a family-centred approach, its application across the western world is only partial and inconsistent (Als et al., 2004; Atun-Einy \& Scher, 2008; Montirosso et al., 2016). Consistency with methodological aspects of research into developmental care may result in the increased confidence and motivation to translate positive findings from these studies into practice.

Next, I reviewed the body of evidence relating to the iatrogenic (hospital-derived) effects of the NICU environment on infant and parental physiological and neurobehavioural outcomes. With the concepts of biological embedding and toxic stress already described, I turned to the literature which refers to trauma-related concepts relating to the NICU environment, specifically the theoretical and interrelated concepts of 'Adverse Childhood Experiences' (ACEs), 'Infant Medical Trauma in the Neonatal Intensive Care Unit' (IMTN) and trauma-informed neuroprotective care.

Recently, scholars have described hospitalisation of an infant in the NICU as a specific neonatal trauma and an important adverse childhood experience (Coughlin, 2017; D'Agata et al., 2017; Maddalena, 2013; Marcellus \& Cross, 2016; Montirosso \& Provenzi, 2015). Emerging from a large seminal study with over 9000 participants in the late 1990s, the ACE concept describes a link between adverse childhood experiences and poor adult health outcomes involving non-communicable disease (Berens et al., 2017; Putnam, Harris, \& Putnam, 2013; Pynoos et al., 2014; Shonkoff, 2016). Further to trauma-informed knowledge, existing literature from the field of paediatric medical trauma has most recently described the concept of IMTN. With its specific focus on raising awareness about the experience of hospitalised infants, 
IMTN is conceptualised as "the intertwined and cumulative early life experiences of stress, parental separation, and pain" (D'Agata et al., 2016, p. 291). The hospitalised infant, therefore, is acknowledged as enduring a traumatic adverse early experience due to a constellation of maternal-infant separation, stress and pain unmitigated by the support of a primary caregiver. These three experiences are "recognized by the brain as a threat to an individual's environment, including physiological, psychological, or emotional demands" (D'Agata et al., 2016, p. 292), and place the individual on a negative trajectory for a raft of poor long-term health outcomes. The concepts of ACEs and IMTN are important, well-described and established theories which inform neuroprotective strategies for the NICU-bound infants and their families, in addition to having huge relevance for healthcare and human rights advocacy.

Neuroprotection stems from "all interventions that promote normal development and prevent disabilities, including organizational, therapeutic, and environmentmodifying measures, such as individualized family-centered developmental care and early intervention programs” (McGrath et al., 2011, p. 109). In 2018, scientific knowledge relating to trauma-informed care in the NICU is well established and whilst a synthesis of related perspectives is outside the scope of this review, it is my conclusion that the theoretical evidence base for its implementation is sound (Altimier \& Phillips, 2016; Ash \& Wiliams, 2016; Coughlin, 2014, 2017; D'Agata et al., 2017; D'Agata et al., 2016; Marcellus, 2014; Marcellus \& Cross, 2016; Sanders \& Hall, 2018).

\subsubsection{Kangaroo Mother Care theory}

Kangaroo Mother Care is the one intervention/method considered foundational to all developmental care programmes, supported by an extremely large empirical knowledge base, with very few gaps in evidence. Established empirical knowledge exists for the safe use of KMC for physiologically stable infants within all settings, as evidenced by large volumes of high quality literature including: three recent Ceochrane reviews (Conde-Agudelo \& Diaz-Rossello, 2014; Conde-Agudelo \& Diaz-Rossello, 2016; Moore et al., 2016); multiple other systematic reviews and meta-analyses (Boundy et al., 2015; Cleveland et al., 2017; Lassi, Middleton, Crowther, \& Bhutta, 2015; Lawn, Mwansa-Kambafwile, Horta, Barros, \& Cousens, 2010); literature reviews (DiMenna, 2006; Feldman, 2004; Hubbard \& Gattman, 
2017; Kearvell \& Grant, 2010; Liu et al., 2007; Moore, 2015; Moxon et al., 2015; Neczypor \& Holley, 2017; Penn, 2015; Seidman et al., 2015; Vesel et al., 2015); and randomised controlled trials (Bergman et al., 2004; Charpak et al., 2017; Feldman, Weller, Sirota, \& Eidelman, 2002; Luong et al., 2016; Morelius, Ortenstrand, Theodorsson, \& Frostell, 2015; Russell, Weaver, \& Vogel, 2015; Sharma et al., 2016a; Sharma, Murki, \& Pratap, 2016b; Tallandini \& Scalembra, 2006). In addition, there is an abundance of qualitative literature reporting parental, nursing, practitioner and systemic findings associated with KMC (Gulla, Dahlo, \& Eilertsen, 2017; Kologeski, Strapasson, Schneider, \& Renosto, 2017; Koopman, CallaghanKoru, Alaofin, Argani, \& Farzin, 2016; Lim, 2018; Morelius \& Anderson, 2015; Niela-Vilen, Axelin, Melender, \& Slantera, 2015; Smith et al., 2017).

Large scale systematic literature reviews and meta-analyses in mainly resourcelimited settings report the safety of KMC in comparison to conventional (incubatorbased) care, including a major reduction in neonatal death for babies less than $2000 \mathrm{~g}$ (Boundy et al., 2015; Conde-Agudelo \& Diaz-Rossello, 2014; Conde-Agudelo \& JL., 2016). For babies receiving KMC, decreased serious neonatal morbidity including neonatal sepsis, necrotising enterocolitis and severe pneumonia was reported by Lawn et al. (2010), in addition to decreased hypothermia, hypoglycemia and hospital readmission (Boundy et al., 2015) and increased exclusive breastfeeding (Boundy et al., 2015; Moore et al., 2016). Based on this evidence, the World Health Organization now recommends the use of KMC for all premature and low birthweight babies as an essential intervention and standard of care for the physiological and psychosocial well-being of babies, mothers, families and communities (World Health Organization, 2015, 2016).

Whilst there are few gaps in the evidence base for the safe implementation of KMC for the majority of babies, the following remain unanswered through scientific inquiry, requiring more research. Firstly, the safety and outcomes of KMC for physiologically unstable infants and infants closest to the edges of viability (Bohnhorst, 2010; Lorenz et al., 2017; Ludington-Hoe, Ferreira, Swinth, \& Ceccardi, 2003). Secondly, research on the safety and suitability of KMC for infants undergoing intensive medical treatments. Next, whether there is a dose-response relationship between KMC duration and infant/caregiver outcomes (Bergman, 2015; Boundy et al., 2015; Moore et al., 2016). In addition, there are unanswered questions 
about how to provide KMC for babies whose parents are unavailable as primary caregivers. Lastly, the evidence relating to the implementation methodologies enabling quality improvement of KMC in high income settings, is sparse. The importance of developing a local 'picture' of knowledge, practice and outcomes within individual and national units was highlighted by a recent retrospective study comparing preterm infant outcomes between Australian, New Zealand and Canadian NICUs. The results serve as an example of the importance of regional and local differences in practice and outcomes for hospitalised infants. From this study, several medical outcomes including severe retinopathy, neurological injury, necrotising enterocolitis, late-onset sepsis and chronic lung disease were shown to vary significantly between locations (Hossain et al., 2015). The conclusion from this study was that Australasian infants "fared better in most measures... maybe related to differences in tertiary service provision, referral and clinical practices" (Hossain et al., 2015, p. 882).

Whilst there has historically been more evidence from resource-limited countries for the safe and effective use of $\mathrm{KMC}$ as an alternative to conventional care for the majority of babies (Bergh et al., 2016; Bergh et al., 2014; Bergh et al., 2012b; Charpak \& Ruiz, 2016; Luong et al., 2016; Moxon et al., 2015; Uwaezuoke, 2017; Vesel et al., 2015), the last decade has produced an increasing number of studies and expert opinion in support of upscaling of KMC that have emerged from the communities of the developed 'global North' (Abadia-Barrero, 2018; Boundy et al., 2015; D'Agata et al., 2017; Nyqvist et al., 2010b; Nyqvist, 2016; Nyqvist et al., 2013). Widespread national and international consensus now exists for "universal use of Kangaroo mother care" with stable preterm and low birthweight infants greater than 27 weeks gestation (American Academy of Pediatrics et al., 2016; Baley \& COMMITTEE ON FETUS AND NEWBORN, 2015; Lassi et al., 2015; World Health Organization, 2015).

Local literature produced by Australian and New Zealand scholars consists of just six papers using various methodologies, five of which were focused on the topic of maternal-infant attachment physiology and KMC. Researchers in Australia conducted a literature review of qualitative studies on the effect of hospitalisation and infant ill health on attachment processes within neonatal units. Their conclusion was that KMC was one of three major methods through which nurses could support 
maternal-infant bonding, one key recommendation being that (Kearvell \& Grant, 2010, p. 81):

...nurses need to minimise mother and infant separation by promoting mother-infant interaction through kangaroo care... increased knowledge and evidenced based research is needed to help implement these practices to assist the mother-infant dyad.

Primary quantitative research of Australian mothers showed a lack of maternal attachment as one of three main predictors of maternal-infant relational avoidance patterns, with increased risk of increased maternal psychological symptoms and poor long-term infant mental health outcomes for their babies (Evans et al., 2012). A common limitation of much of the primary literature was noted as: the self-reported nature of the data (and therefore the potential for bias based on skewed populations) (Roberts, Paynter, \& McEwan, 2000); small sample sizes (Evans et al., 2012); and poor response rates and therefore low generalisability (Chia et al., 2005). Primary research in the NICUs of New Zealand with respect to preterm outcomes other than mortality consisted of one paper reporting a multi-centred trial of FICare, one measure of which was skin-to-skin care (O'Connor, 2016). This trial wasn't concluded and therefore the findings have not yet been published. There is a notable gap in research of KMC in the NICUs of Aotearoa New Zealand.

\subsection{REVIEW THREE: IMPLEMENTATION OF KMC IN THE HIGH-INCOME NICU}

Knowledge relating to the topic of translation of KMC theory (or the frameworks that support it) into NICU practice within high-income countries could be described as scant: "there is a lack of research examining how family-centered care can be successfully implemented in practice" (Skene et al., 2015, p. 657). Whilst this statement refers to the implementation of a developmental (family-centred) care programme, the same could be said for studies detailing the implementation of KMC in the high-income setting. In contrast, KMC scholars working in the developing middle-income South African environment began action research studies on implementation and conceptual tools for upscaling of KMC in the early part of the $21^{\text {st }}$ century (Bergh et al., 2005; Bergh \& Pattinson, 2003; Bergh et al., 2008). These tools were then rolled out into large KMC upscaling efforts over the next decade in other African countries such as Uganda, Malawi, Rwanda, Mali and Ghana (Bergh et al., 2014; Bergh et al., 2012b) and later in Asia (Bergh et al., 2016). 
Conclusions from KMC implementation research in low-income countries suggest that strategies should be contextualised to the local KMC environment (Bergh et al., 2008). In addition, the consideration of both quantifiable (institutional, management, infrastructure, staff and patients/parents) and non-quantifiable (institutional ethos and climate, commitment and care, respect for human rights) factors is essential. This is due to the idea that "a major paradigm shift from key role players and management" would be required to shift the role of primary caregiver back from the healthcare worker to the mother (Bergh \& Pattinson, 2003, p. 713). The scholars involved in $\mathrm{KMC}$ implementation within the developing countries, where this literature emerged from, noted the ongoing difficulty for upscaling of KMC, despite the heavy implementation efforts of many within their context: "individual institutions still struggle to get KMC institutionalised in a sustainable way" (Bergh et al., 2012a, p. 38). Grass roots, academic and policy pathways are recommended for the local and contextual innovation of educational models in support of the KMC upscale (Bergh et al., 2012a).

I conducted a search of the recent KMC implementation literature from high-income countries since the year 2000, using the key search terms: Kangaroo Mother Care, implementation, neonatal intensive care unit and high income/developed countries. Literature from 15 studies was found from countries including Italy, Sweden, the United States, Finland, Israel, France and Australia. Of those, the majority were literature reviews or expert commentaries on the empirical evidence base for KMC implementation, guiding principles and their recommendations, rather than evidence of methodological processes for the implementation of KMC theory into practice (Brett, Staniszewska, Newburn, Jones, \& Taylor, 2011; Campbell-Yeo, Disher, Benoit, \& Johnston, 2015; Lawn et al., 2013; Nyqvist et al., 2010b; Nyqvist et al., 2013; Penn, 2015; Roue et al., 2017; Rutgers \& Meyers, 2015). One study was a peer-reviewed publication of a suggested PAR protocol for the implementation of a family-centred practice in England which proposed the inclusion of KMC. The purpose of this study was to "contribute new knowledge about approaches to actively involve parents in the care of their infant on neonatal intensive care units" (Skene et al., 2015, p. 658). There have been no published reports of this study, to date.

Literature relevant to my own proposed NICU study was found in five studies (six papers) conducted between 2005 and 2015. A diverse range of research methods 
were adopted which included qualitative focus groups within a randomised controlled trial, quality improvement tools such as the Iowa model, and an evidenceinformed KMC protocol design. Researchers examined staff perspectives from four NICUs in Sweden which used change teams to implement a KMC guideline. KMC evidence was deemed important but not entirely adequate for the implementation of sustained change, requiring other supportive factors based on context and facilitation (Wallin, Rudberg, \& Gunningberg, 2005). For the change teams in the Swedish units, leadership and organisational values were suggested as even more important to KMC guideline implementation than other factors (Wallin et al., 2005, pp. 70, 71):

conditions [of workload and organisational resources] are most likely a reflection of organizational values and how improvement efforts are organized which again highlights the role of leadership.

Facilitation of this Swedish KMC implementation study was deemed effective across four units due to its "enabling approach" (Wallin et al., 2005, p. 71), whereby staff were supported as a team to work out and implement a strategy appropriate to their own contexts. In addition, KMC knowledge dissemination was facilitated using a champion or clinical opinion leader who was respected by the team, researchers concluding that "existing organizational values and clear leadership support seemed to have worked every bit as well as the external intervention" (Wallin et al., 2005, p. 71).

Nursing researchers in the United States adopted a quality improvement tool known as the Iowa method to shift the model of nursing care in their postpartum unit. Traditionally, mothers and infants were often separated in their unit and there was an aim to shift to a mother-baby 'couplet care' model which included early and continuous skin-to-skin contact between the pair. Although this study did not involve a NICU population, the study was relevant to this one due to the requirement for an organisational culture shift to accomodate the new model of care. Prior to the study, researchers acknowledged that "Because this change represented a culture shift, ensuring success required significant research, planning and preparation" (Brockman, 2015, p. 493). In common with my own suggested research, change theory, evidence based practice and translation of theory into practice within a complex social system were highly informing of this study in one United States setting. Bold leadership (including a nurse leader who believed in the change and remained engaged with the project), inclusive and detailed planning, engagement 
with bedside staff and financial considerations were all deemed important to "ensure the change is hardwired for long-term sustainability" (Brockman, 2015, p. 501).

An Italian paper was examined for my review due to its findings from a high-tech setting which embarked on contextualising a KMC protocol for use in its level three NICU. There was an impetus by this group to individualise a KMC clinical guideline suited to their particular setting, because of the inconsistency of KMC application within their context. A KMC protocol was designed based on the recommendations from the Expert Group of the International Network on Kangaroo Mother Care, including the current evidence base as well as staff opinion, practical and experiential considerations (Davanzo et al., 2013). Authors conclude that whilst individualised protocols are important for facilitating KMC implementation, additional aspects of the physical environment and humanistic considerations are required for the optimisation of KMC (Davanzo et al., 2013).

Research on KMC within the NICU most local to the New Zealand context was conducted in Australia, using a survey and qualitative interviews of neonatal nurses' attitudes toward and practices of KMC. The findings from this study were strongly in line with other global literature, noting that nurses support the use of KMC in theory, and mostly agree with its benefits in low birth weight babies (Chia et al., 2005). Notable KMC barriers were also consistent with those from large global systematic reviews, including lack of clear protocols, high workloads, lack of parental and staff education and insufficient organisational support (Chia et al., 2005). As with the other literature in this review, aspects of organisational culture were pointed to in a cursory way: "It is well established that attitudes are a major determinant of behaviour... the NICU environment often limits the parent's ability to care for their infant and to practice K(M)C" (Chia et al., 2005, p. 25). There was, however, no further analysis or discussion about which particular attitudes, behaviours, or organisational effects were implicated as barriers to KMC. In conclusion, the authors state the need for implementation strategies which overcome the barriers to KMC identified by neonatal nurses, with no further comment on which particular strategies are recommended.

The last relevant study was a single-centre randomised controlled trial conducted in the United States, researching the effects of a developmental care method called 
Family Nurture Intervention, reported in two published papers. The intervention is proposed to ameliorate the negative effects of prolonged caregiver-infant separation through the use of calming methods which may or may not involve skin-to-skin contact. The relevance of this study, therefore, was due to its setting within a high income NICU and its acknowledgement of "increasing calls for novel evidencebased interventions that can limit or overcome long-term developmental morbidities that accompany preterm birth" (Welch et al., 2013, p. 2). It was noted by researchers that parental involvement was well tolerated and integrated into hospital routines for the duration of the implementation study, a common concern for researchers working in the complex NICU environment. This may encourage researchers and participants in the future to consider more implementation studies involving parent participants, given that "families remain an under-utilized resource for infant care (within the NICU)” (Welch et al., 2015, p. 1209). The more recent paper from this study identified improved neurodevelopmental outcomes across several domains in toddlers who had received the intervention as babies in the NICU. The scholars claim this to be the first NICU randomised controlled trial to show a positive effect of a nurture-based intervention (Welch et al., 2015). As with the other studies in this review, a nod to organisational effects on the implementation of developmental care interventions was made " ... cost of implementation, demand on resources... resistance to change..." (Welch et al., 2013, p. 2), yet not inquired into further. In conclusion, whilst there is plentiful evidence for the recommendation of implementation of KMC into all NICU settings, including those of high income countries, there are only small amounts of literature detailing the processes by which this can be achieved. The majority of scholars note the importance of the contextualisation of implementation principles to each individual setting, recommending the inquiry into organisational, leadership, workforce and parental perspectives. Methodological recommendations for KMC implementation, including effective methods for negotiating the complexity of the NICU environment and care culture in high income countries are nearly absent. The inquiry into the effects of organisational culture on KMC, whilst acknowledged as being a significant factor in its implementation, remains a large gap in the evidence-base for effective and sustained upscale of this intervention. 


\subsection{REVIEW FOUR: SOCIAL CONSTRUCTION OF NEONATAL INTENSIVE CARE \& INSTITUTIONAL RACISM}

I conducted two separate searches of the literature relating firstly to social construction of the NICU, and secondly to institutional racism within New Zealand's healthcare context. I used Pubmed; CINAHL Complete; ProQuest Nursing and Allied Health; and, Te Waharoa Victoria University of Wellington databases for the two reviews. Searches used key terms of: 'sociocultural', 'social construction', 'neonatal intensive care unit'; 'neonatal care' and 'families' for the first review. This was followed by 'institutional racism', 'healthcare' and 'New Zealand' search terms for the second review. I also supplemented the search by referring to reference lists of included studies for literature that was highly relevant and didn't show in the initial searches. The search limits were for both reviews were:

i. Articles published between 2000 and 2018;

ii. Articles written in English;

iii. Articles from peer-reviewed journals;

iv. Articles which included the search terms within the abstract, main body or reference sections;

v. Articles published worldwide;

vi. Articles based on research conducted in New Zealand and Australia

The first search relating to sociocultural construction of neonatal care found 49 papers across the four databases. Exclusion was based on duplication of papers, lack of relevance and/or access to the paper, after which there were 26 papers included in the review. The second review of health institution racism in New Zealand included 43 papers of relevance, although none were directly related to the NICU setting. The literature varied widely from expert commentary reports through to original qualitative research on both review topics, from diverse disciplines such as occupational therapy, social work, psychology and medicine. Both reviews focused on the major concepts that emerged from literature in relation to firstly, social construction of the NICU, and secondly, institutional racism and healthcare in Aotearoa New Zealand. 


\subsubsection{History of neonatal intensive care}

In order to understand the context of this study through a constructivist lens, some historical background on the development of the neonatal unit is important (Carnevale, 1998; White, 2011). In the early 1900s, perinatology as a specialist field of medicine gained prominence. Premature infant care, including gavage feeding with donated human milk in nurseries became commonplace within the generalised hospital setting (Gartner \& Gartner, 1992). Incubators for human infants, modelled on chick incubators, began emerging in Europe from the 1880s and were brought to the awareness of the general public by the famed 'kinderbrutanstalt' exhibitions of live premature infants in incubators. Care of preterm infants within the specialised neonatal intensive care environment as we know it today emerged in the United States in the early 1960s. At that time there were laws actively prohibiting such units, due to the lack of precedents for organisation of care, along with the justifiable fear of cross-contamination by Staphylycoccus aureus (Gluck, 1992). In the earliest days of the NICU, the only source of nutrition for the infants was human milk, often donated by wet nurses, whilst mothers were considered either non-essential or dangerous to their infants (White, 2011).

As childbirth moved away from the home environment in the early 20th Century, two major care paradigms have evolved for the growing number of hospitalised infants, largely without scientific basis (Bergman, 2014; Phillips, 2013). Traditional, or nursery-based care dominated the period of NICU development from the 1960s through until the late 1980s (Gartner \& Gartner, 1992; Gooding et al., 2011; White, 2011). It was characterised by task- and protocol-based medical treatment of babies by nursing and medical staff, most usually with prolonged maternal-infant separation (McGrath, Samra \& Kenner, 2011). Initially, the major focus of traditional care was to lower mortality and had little focus on psychosocial support or long-term developmental outcomes for infant or family. As survivability of preterm newborns at lower gestations and birthweights increased, clinical experience of the healthcare teams, parental observations and emerging research on the possible deleterious effects of the NICU environment developed (Barbosa, 2013; Gooding et al., 2011; McGrath et al, 2011; White, 2011). The second paradigm of care was broadly termed 'developmental care'. This paradigmatic shift, including the family-centred frameworks that support it, are currently advocated for by a proportion of parents, 
researchers and clinicians in the field (Als, McAnulty, \& Gloria, 2011; Hall et al., 2017; Landzelius, 2006; Phillips, 2013; White, 2011). Despite advocacy for increased developmental care practices; however, the care of infants within incubators, with prolonged maternal-infant separation, remains a predominant care practice for the majority of infants around the world where financial and physical resources allow for it (White, 2011). In the broader societal context, this was accompanied by increasing medicalisation of childbirth and maternity care within the hospital setting (Flacking \& Dykes, 2013; Gooding et al., 2011). This historical perspective brings us to the 21 st century literature relating to the social construction of the NICU.

\subsubsection{Social construction of the NICU}

\section{Neonatology: a medical subspeciality}

Neonatology began as a medical specialty that was focused on life-saving interventions for premature and sick babies. The late 20th century was a time when there was necessary focus on developing technology and care in support of infant thermoregulation, respiration and preventing infection (Fegran, Helseth \& Slettebo, 2006; Gooding et al., 2011). More recent knowledge reports that inherent stressors within the NICU environment pose risks to infant and family development, and the integration of more holistic, individualised principles of caring are required to improve outcomes for babies (Atun-Einy \& Scher, 2008; Barbosa, 2013). Although there has been a reported shift in attitude by some practitioners to embrace parental partnership and family-centred care (FCC), power relations remain assymetrical and FCC implementation problematic (Atun-Einy \& Scher, 2008; Barbosa, 2013;

Ballweg, 2001; Fegran et al., 2006; Gooding et al, 2011; Hall, Phillips, \& Hynan, 2016; Kuhn, Sizun \& Casper, 2018; McGrath, Samra \& Kenner, 2011).

Inquiry into the social construction of knowledge at the structural levels of society and within the NICU reveals the predominance of biomedical theory and discourse as 'truth', with practitioner voice privileged as expert knowledge (Carnevale 1998, Fegran et al., 2006; Golden, 2017; Landzelius, 2006). Whilst scant literature exists pertaining specifically to the NICU-context, it is reasonable to state an assumption based on knowledge from the modern hospital system, that the normative 
hierarchical culture of the NICU constitutes an ongoing barrier to developmental care and nurturing practices (Atun-Einy \& Scher, 2008; Griscti et al., 2017).

Neonatology remains posititioned as a specialty discipline within the medical field, amidst the "crisis" of life-or-death (Price \& Miner, 2008, p. 72), resulting in the normalisation of NICU-experience as a clinically managed "extraordinary life situation" (Flacking and Dykes, 2013, p. 1). Whilst this medical discourse may be understandable to the observer, one effect is to contribute to marginalisation of family narratives and non-medical interventions through the clinical management of babies via the "machinery of biopolitics" (Lanzelius, 2006, p. 669). This results in the maintenance of the normative biomedical order, disempowerment of parental knowledge and authority, and sidelining of what may be considered the more 'mundane' acts of nurture (Landzelius, 2006; McGrath et al., 2011; Redshaw, 2014; Spinelli, 2016). To counter this phenomenon, "Social demands on mothers in particular resulting from the powers of medicine create the need for the construction of new identities" (Redshaw, 2014, p. 133).

\section{Nurture in the NICU}

Collaborative, developmental and psychosocial support of babies and families in the NICU was widely reported by parents and staff as being important to the short- and long-term wellbeing of infants and their families (Barbosa, 2013; Fegran et al., 2006). That said, NICU admission remains an important negative predictor of nurturing activities such as exclusive breastfeeding and maternal-infant attachment, and "very few studies have focused on the psychosocial needs and opinions of parents" (Alves, Rodrigues, Fraga, Barros, \& Silva, 2013, p. 1511-1512).

Several parental perspectives did, however, emerge from the most recent literature. One perspective from parents was the acknowledgement of breastfeeding in the NICU as a "gendered, embodied experience... perceived, simultaneously, as a biological and social phenomena" (Alves et al., 2013, p. F513), important for nutrition, bonding and the development of parental roles (Alves et al., 2013). Another was the importance to biological parents of entering into NICU-parenthood "by a process awakened by the skin-to-skin [SSC] interaction with the infant" (Anderzen-Carlsson, et al., 2014, p. 14). In other words, parenting was not solely pre-programmed (biological), but more a social construction requiring environmental 
support for embodiment of the role. As well as the need for physical proximity, becoming a mother was described as a phenomenon relating to spiritual and philosophical values (Anderzen-Carlsson et al., 2014). Interestingly, whilst SSC was considered important for their child's development in NICU and desired by most, the fear of hurting their baby led to some parents' being emotionally drained by the practice (Anderzen-Carlsson et al., 2014).

Relationship-based, family-centred care as 'best practice' within the NICU was a prevalent discourse within the literature (Gooding et al., 2011; McGrath et al., 2011; Price \& Miner, 2008). FCC holds space for the importance of nurturing activities for medically fragile babies such as breastfeeding (Alves et al., 2013; Flacking \& Dykes, 2013); providing SSC and positive touch (Altimier, 2015; Anderzen-Carlsson, Lamy, Tingvall, Eriksson \& Eriksson, 2014; Shin \& White-Traut, 2007; Spinelli et al., 2016); performing nappy-changes and bathing (Price \& Miner, 2008); and parents becoming the primary caregivers of their babies (Barbosa, 2013; Fegran et al., 2006; McGrath et al., 2011). Due to the complex nature of nurturing hospitalised babies, there was general consensus relating to the necessity for primary caregiving by parents, assisted by partnership with staff and allied support (Altimier \& Phillips, 2016; Pridham et al., 2006; Spinelli et al., 2016). Within the organisational context, parental and interdisciplinary partnership was recommended, underpinned by principles of collaboration, power/knowledge-sharing and guided participation (Landzelius, 2006; McGrath et al., 2011; White, 2014).

In addition, the impacts of the environment and effects of NICU 'place' on hospital social life, functional NICU-relationships and knowledge/information-sharing were important factors - found as both facilitators and barrriers to parents' experience of nurturing their baby (Altimier, 2015; Alves et al., 2013; Flacking \& Dykes, 2013; Gooding et al., 2011; Kuhn, et al., 2018; White, 2011). Institutional, multi- and interdisciplinary collaboration, family partnership, and methodological means for advancing family-centred developmental care was called for (McGrath et al., 2011; Price \& Miner, 2008).

On the topic of paradigm, it was noted by some scholars that the dominant biomedical discourse was overlaid on nurturing activities such as breastfeeding and 
physical contact within the NICU in ways that minimised the importance of nurture, per se (Alves et al., 2013, p. F515-516):

... most of interventions to promote breast milk supply in NICU have focused on the physiological benefits of breastfeeding. Even those that value the skin-to-skin contact do it in the sense of improving nourishment, health outcomes and the duration of breast milk supply. However... parents often valued breasfeeding as an opportunity to hold and connect with infants.

Biomedical discourse also underpinned much of the literature reporting on NICU ecology, framing the infants' needs through medical and neurodevelopmental lenses, rather than humanitarian perspectives (Charpak \& Ruiz, 2016; Kuhn et al., 2018; Lester et al., 2014; Vohr et al., 2017). In response, technologies for enhancing the NICU environment in favour of individual families, rather than the needs of the neonatal team, have been described, such as the single-family room design (Lester et al., 2014, Meredith, Jnah \& Newberry, 2017; Vohr et al., 2017, White, 2011).

Scholars increasingly recommend that balancing and integrating psychosocial support with medical care is crucial to improved outcomes for babies and families, said by Hall and colleagues (2016) in this way: "Providing psychosocial support to families in the NICU should not be considered an optional activity, but should be the foundation upon which NICU staff provide excellent medical care" (p. 72). To this end, more knowledge is required about the people who receive NICU services; their physical, emotional, cultural and spiritual needs (Alves et al., 2013; AnderzenCarlsson et al., 2014; Flacking \& Dykes, 2013; Kuhn et al., 2018; Nazareth \& Santos, 2014; Pineda et al., 2012; White, 2014); and mechanisms that enable more humanitarian, holistic guidance and support (Charpak \& Ruiz, 2016; Pridham et al., 2006; White, 2011).

\section{The people who require neonatal intensive care}

Sociocultural contexts and concepts are hugely informing for people who use NICU services, and culturally relevant care is widely recognised as a top priority (Auslander, Netzer \& Arad, 2003; Lee \& Weiss, 2009; Nazareth \& Santos, 2014). The literature I examined represented culture as the shared beliefs, customs and values of any group that organise their thoughts and decision-making, often intergenerationally (Nazareth \& Santos, 2014). There were also sociocultural responses from within the wider societal milieu that parents were required to navigate for their and their babies' wellbeing (Redsaw, 2014). For example, some women perceived a 
strong social prejudice within their ethnic group against their hospitalised infants and adopted behaviours to deal with this complexity (Lee, Norr \& Oh, 2005; Shin \& White-Traut, 2007).

Sociocultural factors affecting people who experience the phenomenon of NICUcare are known to be diverse, requiring insight on a case-by-case basis (Nazareth \& Santos, 2014). Where multicultural populations were present in the NICU setting, it was important to parents that practitioners spoke their language, and had an understanding of how to communicate according to the norms and preferences of their culture (Auslander et al., 2003; Lee \& Weiss, 2009). Broader sociocultural determinants affecting peoples lives also impacted on their experience in the NICU and may include economic status, racism, access to transportation and basic needs resourcing, family structure and education (Nazareth \& Santos, 2014; Vohr et al., 2017). Some demographics were over-represented in their need for NICU hospitalisation and therefore more pointed knowledge and support for these groups was required, particularly where they represented a minority group. In New Zealand, for example, women aged less than 20 or over-35 were more likely to have a baby who was hospitalised; similarly if a woman identified as Māori or Indian, or was from economically-deprived areas (MOH, 2017). For Māori, racism has a known impact on their experience of, and outcomes from, the healthcare system in New Zealand, a factor explored more fully in the second review.

From a psychological perspective, women were reported to have difficulty with the transition to mothering a hospitialised infant, the suggestion being that the construction of maternal identity (and in turn, bonding) was delayed or disrupted by medical crisis (Spinelli et al., 2016). It was important to women that NICU staff did not usurp their parental role (Spinelli et al., 2016). One way to appreciate women's perspectives and to place the family firmly at the centre of the social world of the NICU was through capturing parental narrative and move them "from the periphery to the centre of medicine" (Redshaw, 2014, p. 125). Models of nursing that inquire into sociocultural factors affecting people, such as the transcultural nursing method, were also recommended (Nazareth \& Santos, 2014).

Most importantly, it is within relational sociology that the agency of the infant must be considered, as an interdependent being in need of biopsychosocial support that 
medical care, alone, cannot offer (Redshaw, 2014; White, 2014). To achieve widescale recognition of this, a paradigm shift is required, to an understanding of the importance of the social interdependence of babies and families, described this way by Redshaw (2014, p. 133):

To consider the infant body as a social body constituted within a network of social relations and not merely the passive biomedical body of medicine... Infants have a being that requires a network of relations to support it and the wider narrative of the family and community to develop within.

\subsubsection{Institutional racism in New Zealand}

Institutional racism is a major public health issue in Aotearoa New Zealand (Came \& Griffith, 2018; Barton, 2018; Kearns et al., 2009). Institutional (structural, systemic) racism refers to "differential access to material resources and power determined by race which advantages or privileges one sector of the population while disadvantaging or discriminating against another" (Came \& Humphries, 2014, p. 98). It persists as a major avoidable determinant of health inequities around the world and constitutes a public health issue for many communities (Came, McCreanor, Doole \& Rawson, 2016; Came \& Griffith, 2018; Foxall, 2013; Jehonathan, Cormack, Harris \& Paradies, 2017; Johnstone \& Kanitsaki, 2010; Kearns, Moewaka-Barnes \& McCreanor, 2009). Indigenous peoples are disproportionately affected by structural racism (Barton, 2018; Curtis, 2013; Grigg \& Tracy, 2013; Walker, 2017; Wilson, 2012; Zambas \& Wright, 2016). Māori, the indigenous people of Aotearoa New Zealand, were reported in the literature as enduring some of the worst levels of racial discrimination, marginalisation and health inequities in the world (Barton, 2018; Came \& Tudor, 2016; Denison et al., 2018; Harris et al., 2006; Harris, Stanley \& Cormack, 2018; Oda \& Rameka, 2012; Scott, 2014; Theunissen, 2011).

Racism is a complex ethical, moral and human rights issue in healthcare (Came \& Humphries, 2014; Johnstone \& Kanitsaki, 2010), not least because it "marginalises denigrates, excludes" (Kearns et al., 2009, p. 128). And, through a social model lens, it creates a barrier to a standard of living afforded to the dominant, privileged group (Human Rights Commission, 2012; Theunissen, 2011; Zambas \& Wright, 2016). Institutional racism determines, and is contributed to by many factors that affect health outcomes, including: colonisation (Zambas, 2016); multi-level discriminatory 
policy and practice (Came, 2014; Came \& Humphries, 2014; Manson, 2012); differential exposure to socioeconomic determinants (Harris et al, 2006); occupational risk factors (Denison et al., 2018); child protection practices (Hyslop, 2018); health service utilisation and access (Came, 2014; Harris et al., 2006; Jehonathan et al., 2017; Manson, 2012; Zambas \& Wright, 2016); physical and mental health outcomes and longevity (Barton, 2018; Came, 2014; Curtis, 2013; Stoner et al., 2015; Wilson \& Baker, 2012); barriers in healthcare education (Barton, 2018; Foxall, 2013); and media representation of Māori (Barton, 2018; Nairn, Pega, McCreanor, Rankine \& Barnes, 2006).

Whilst the concepts and frameworks of cultural safety have been active within nursing for three decades in this country (Richardson 2004, 2010), scholars assert the urgent need for increased confrontation of racism through anti-racism praxis and consciousness-raising (Barton, 2018; Came, 2014; Came \& Griffith, 2018; Pack, Tuffin and Lyons, 2016; Spence, 2005). Māori-centred models of care that encompass holistic and spiritual wellbeing (Manson, 2012; Richardson, 2004; Stoner et al., 2015, Theunissen, 2011), bicultural praxis underpinned by Te Tiriti o Waitangi (Came \& Tudor, 2016), equity-focused care (Browne et al., 2016; Scott, 2014; Seneviratne et al., 2015) and ongoing monitoring of racism through a social justice lens, are all required (Barton, 2018; Harris et al., 2018).

\section{Factors contributing to institutional racism}

The societal effects of colonisation for the people of Aotearoa, where "'White' cultural capital dominates" (Reid, Cormack \& Crowe, 2016, p. 143), are increasingly being brought to light: "colonisation traumatises, harms, kills and dehumanises" (Emery-Whittington \& Te Maro, 2018, p. 18). Historically and ongoing, displacement of connection to their land, language and culture (including rongo $\bar{a}-$ Māori medicine), with subsequent fracturing of economic systems, are impacts that have had major detrimental effects on Māori health and wellbeing (Came \& Tudor, 2016; Emery-Whittington \& Te Maro, 2018; Kearns et al., 2009; Stoner et al., 2015; Zambas \& Wright, 2016). Maternity scholars Grigg and Tracy (2013) describe it this way (p. e59):

the ravages of colonisation on Māori... when combined with ongoing institutional racism, has resulted in Māori being economically, culturally and socially dispossessed and deprived. Currently New Zealand is a European/western-dominant 
culture and society, which still faces the challenge of learning to share power between the Treaty Partners.

Other systemic factors continue to contribute to racist impacts. Educational barriers in nursing for Māori students have been identified, with poor recruitment and retention strategies, lack of cultural competence, and insufficient academic and financial support found (Foxall, 2013). Some scholars discussed how neoliberalism contributes to racially discriminating practices, such as those present in New Zealand's child protection practices (Bryers-Brown \& Trundle, 2017; Came, 2014; Hyslop \& Keddell, 2018; Nairn et al., 2006). Currently, Māori children are overrepresented in all areas of child-protection work, one theory positing that it is the social construction via neoliberal ideology that places "parental responsibility for child well-being in a society riven by systemic social inequality" (Hyslop \& Keddell, 2018, p. 2). The discourse of indigenous children's risk and abuse are enmeshed in a racialised narrative, underpinning child protection systems and structural issues of racism and inequality (Hyslop \& Keddell, 2018). Hyslop \& Keddel (2018) assert (p. 6):

The over-representation of Māori in the A-NZ [Aotearoa New Zealand] child protection system is largely a product of how the historical legacy of colonisation, strutural inequality and institutional racism continues to play out in the lives of individuals and families... the philosophy is that individuals can be targeted and remoralised or their children can be placed elsewhere if intervention is unsuccessful.

It is noteworthy to speak of New Zealand media's contribution to racist child protection practices, with respect to the conflation of Māori ethnicity and state dependency with immoral, gendered violence (Hyslop \& Keddell, 2018). The discourse of race in Aotearoa New Zealand, represented by the media, is arguably one where interpersonal and internalised racism has been institutionalised through its normalisation of colonial ideology (Nairn et al., 2006). Inquiry at the intersection of media, race and health in Aotearoa New Zealand concluded (Nairn et al., 2006, p. 191)

... we have shown that the colonists created a racist society and developed discourses to help naturalize and render invisible that oppression. We have sketched the media contribution to promoting and maintaining Pakeha domination.

The presence of implicit and unconscious bias and negative racial stereotyping is undoubtedly an ongoing mechanism for the maintainence of racially-induced inequities (Barton, 2018; Reid et al., 2016). 


\section{Determinants of health inequities for Māori}

Structural racism within New Zealand's healthcare system has been identified across multiple sites through mechanisms such as mono-cultural policy-making and funding practice, and the marginalisation of Māori worldview (Barton, 2018; Came, 2014, Came \& Humphries, 2014; Curtis, 2013; Hill et al., 2010). Most recently, scholars critiquing the New Zealand Health Strategy (2016) identified that systemic, multilevelled engagement with Te Tiriti o Waitangi as a platform for adressing inequities between Māori and non-Māori whilst necessary, remains absent (Came, McCreanor, Doole \& Rawson, 2016).

Health service inequities are the largest determinant of poorer disease outcomes for Māori (Seneviratne et al., 2015). For example, Māori are more likely to suffer fatal consequences from: cardiovascular disease, accidents and suicide (Curtis, 2013; Harris et al., 2018; Oda \& Rameka, 2012; Stoner et al., 2015); chronic kidney failure (Huria, Palmer, Beckert, Williman \& Pitama, 2018; Walker et al., 2017); and various cancers (Hill et al., 2010; Seneviratne et al., 2015). Māori children are 20 times more likely to contract rheumatic fever, a now-rare disease in the rest of the developed world (Manson, 2012). Māori women are more than twice as likely as Pākeha women to die from breast cancer (Seneviratne et al., 2015) and, on average, live nine years less than non-indigenous women (Wilson, 2012). In addition, Māori mental health disorders are more prevalent than non-Māori and linked to the effects of colonisation (Wilson \& Baker, 2012).

Other mechanisms also exist through which structural racism affects Māori health. Occupational risk factors associated with low-skilled and high-hazard jobs are disproportionately experienced by Māori (Denison et al., 2018). Preliminary evidence suggests that working conditions are worse for Māori than non-Māori, with associated increased risk of physical and psychological harm through various means (Denison et al., 2018). A myriad of wider determinants also contribute to Māori health inequities within sectors such as education, finance, justice, transport and social development (Barton, 2018; Human Rights Commission, 2012).

\section{Eradicating racism will improve health inequities: future directions}

Racism remains a "potent but neglected determinant of health" (Kearns et al., 2009, p. 123). Health services and the state authorities who govern them are ethically, 
morally and legally bound to embed evidence-based frameworks that promote health care equity for all people (Johnstone \& Kanitsaki, 2010). This is especially so for their indigenous citizens who experience poorer health outcomes at least in part due to structural racism and cultural incompetence (Browne et al., 2016; Came, 2014; Came \& Tudor, 2016; Jehonathan et al., 2017). Societal validation of the trauma imposed upon indigenous peoples by colonialism is considered by many to be necessary for "cultural healing" (Zambas \& Wright, 2016, p. 400). Whilst there are similar trends across the world for indigenous people, contextual knowledge is important for understanding the experience of Māori and non-Māori in New Zealand.

The British Crown as well as political and healthcare leaders are bound by a foundational commitment through Te Tiriti o Waitangi to priorititse and address Māori health inequities (Came, 2014; Came \& Tudor, 2016; Oda \& Rameka, 2012). This is recommended through the lenses of colonialisation and biculturalism using Māori-centred research, policy and practice frameworks (Barton, 2018; BryersBrown \& Trundle, 2017; Came, 2014; Oda \& Rameka, 2012; Richardson, 2010). Whilst inclusion of Māori participation with central and regional health legislation is mandated in New Zealand (unlike the situation in Australia and Canada), the mechanisms are variably embedded and accounted for (Lavoie, Boulton \& Gervais, 2012; Spence, 2005; Wilson, 2012). “Anti-racism pathways" (Came, 2014, p. 218) are both possible and necessary, and include ongoing decolonisation processes (including systematic education and application of Te Tiriti o Waitangi) and balancing of power through tino rangatiratanga - Māori sovereignty (Came, 2014; Came, Cornes \& McCreanor, 2016; Came \& Tudor, 2016; Emery-Whittington \& Te Maro, 2018; Oda \& Rameka, 2012; Stoner et al., 2015). Some argue that it is not enough to focus on the "symptoms of poverty and the distress that is causes" (Came \& Tudor, 2016, p. 189), rather principled, politicised action- and policy-based health promotion to develop Māori-centred services based on Māori knowledge and participation (Came, McCreanor \& Simpson, 2017; Came \& Tudor, 2016; Scott, 2014; Zambas \& Wright, 2016).

An important established pathway for balancing historical and ongoing power differentials for Māori and non-Māori within healthcare lies in nursing education, through the concept of cultural safety (Richardson, 2004; Richardson, 2012; Richardson \& MacGibbon, 2010; Spence, 2005; Wilson \& Baker, 2012). Cultural 
safety may be realised when there is reflection by practictioners on their personal cultural beliefs, understanding their impacts on the nurse-client relationship, and the incorporation of client's culture in their care plan, as they determine it to be (Oda \& Rameka, 2012; Richardson, 2004). The development of trusting relationships was described as important, Richarson (2012) asserting that "the trust relationship is the crucible within which all other elements of cutural safety are held" (p. 6). In addition, cultural safety must necessarily move to being embedded in institutional practice through education strategies that teach Māori history, race ethics and Te Tiriti o Waitangi (Pack et al., 2016). To do this, there is a need to disrupt more privileged hierarchical and medical discourses in health, the knowledge of which sits uneasily alongside cultural safety discourse (Richardson \& MacGibbon, 2010; Wilson \& Baker, 2012). More focus on practice research and monitoring of primary healthcare experiences of Māori through the lens of cutural safety, is required (Reid et al., 2016; Richardson, 2012).

One aim of bicultural collaboration, therefore, is to "reduce and reverse the constant overlay of non-Māori usages over Māori practices, processes and place that are critical to the maintenance of Māori identities and worldviews" (Kearns et al., p. 127). Within the tertiary education sector, removal of recruitment and retention barriers are required to effectively partner with Māori health students, particularly through enhanced cultural relevance, participation of indigeous staff and mentors, and commuity/funding support (Foxall, 2013). Development and research involving the indigenous health workforce is also required (Wilson \& Baker, 2012). The knowledge that $80 \%$ of primary healthcare patients first meet with nurses, places nursing professionals in a strong position to catalyse and lead change for reducing racism and improving healthcare equity (Manson, 2012; Oda \& Rameka; 2012; Reid et al., 2016; Theunissen, 2011, Wilson, 2008).

Pro-Māori media frameworks are suggested where New Zealand's mass media is required to account for their "pro-settler bias" (Nairn et al., p. 192) and increase bicultural, evidence-based reporting that reflects an intention to deconstruct racism. In addition, the conscious deconstruction of racial stereotyping relating to indigeneity, combined with raising awareness about the positive health effects of 'White' privilege, is required (Reid et al., 2016). In combination, these may impact race health inequities through uniting attitudes and trusting relationships that foster 
meaningful partnership between Māori and non-Māori (Pack et al., 2016; Walker et al., 2017).

Through a constructivist lens, the phenomena of nurturing babies and supporting the psychosocial needs of whānau within the NICU environment is socially constructed, complex and multi-faceted. Neonatology is a relatively recent subspecialty of medicine. It was historically positioned as the authoritative knowledge within the NICU at a time when saving infants' lives was the single-focus of care. Whilst there is an acknowledged paradigm shift toward care models that support biological, emotional and spiritual needs of babies' and their families', translation of psychosocial theory to practice has been slow and inconsistent. Furthermore, while the extreme vulnerability of all patients and support people in the NICU space is unquestioned, indigenous Māori whānau represent priority groups that are pertinent to the New Zealand context, to whom the importance of culturally relevant care is utmost. Breeches of indigenous and human rights, through institutional racism, remains prominent within the experience of New Zealanders, the extent of which is not currently understood in the NICU.

\subsection{SUMMARY AND KNOWLEDGE GAPS}

Knowledge about infant development and attachment are grounded in theoretical and experimental Life Science biology, with theories of biological expectation in the postnatal period currently informing best practice in maternity care. Separation of mother and baby soon after birth and in infancy is established as undesirable, with enduring separation being viewed through a neurophysiological lens as a cause of toxic stress. The NICU environment and culture are universally identified as potential sources of toxic stress for infants, with associated negative long-term outcomes. Principles of biological embedding, the mechanisms of which are not fully elucidated, have emerged over the last 30 years as the process by which adverse childhood experiences such as NICU hospitalisation 'get under the skin' of babies and endure over the whole life course. Current literature describes biological and epigenetic mechanisms by which the environment interacts with the individual to produce negative neurodevelopmental outcomes. While the specific mechanisms are yet to be fully elucidated, the implementation and further research of environmental modification through developmental care practices is reportedly a high priority for neonatal researchers. 
The current literature reports marked variance between DC models, including multiple interventions, within different frameworks, within different settings. Preliminary findings are suggestive of improved infant neurodevelopment for those who received appropriate developmental care, at least in the short to medium term. There is, however, a gap in knowledge and further research is required into which specific developmental care frameworks are cost effective, beneficial and sustainable within the NICU setting. The current evidence suggests that developmental protocols are likely to be highly contextual based on the needs of the individuals, communities and social circumstances in which they are provided.

One intervention that is common to all high quality developmental care models is Kangaroo Mother Care. For KMC, there is compelling evidence of the positive physiological, emotional and cognitive outcomes for the majority of physiologically stable infants. Further evidence is required for the small minority of babies who are physiologically unstable, receiving treatments for conditions such as nonphysiological jaundice and for those whose parents are unavailable for care. In addition, the relationship between the dose-dependent response of KMC associated with positive outcomes requires further research, the findings of which will assist in developing practice and policy. KMC findings from research within NICUs in New Zealand have not been published to date. Global recommendations based on the strength of empirical KMC evidence, suggest generalisability of findings from KMC research from low-income settings to high-income countries (American Academy of Pediatrics et al., 2016, p. 1): "Upon review of the evidence, we agree that KMC provides benefits to preterm and low birthweight infants in high, middle, and low income settings".

The review then moved from the established evidence base for the biological and developmental rationale for ubiquitous KMC application, to the scant literature on implementation methodology within the NICU of high-income countries. The third review identified a large gap within the implementation literature for recommendations on the systematic 'how-to' for the upscaling of KMC in developed countries. Expert opinion, evidence-based guidelines and protocols are common for $\mathrm{KMC}$, yet there is prolific evidence of inconsistent application coupled with a dearth of knowledge on effective implementation methodologies. Implementation evidence from low- and middle-income countries is suggestive of systematic 'grass roots' 
social science approaches to KMC development, some of the most prominent studies using an action research approach (Feucht, Van Rooyen, Skhosana, \& Bergh, 2016). Whilst effective, applied KMC is considered highly contextual within complex NICU systems, implementation principles may be applied to a broad range of settings (Bergh et al., 2012a). Participatory, collaborative and whole-system methodologies have been used in developing countries to capture institutional, practitioner and parent/patient buy-in, which inquire into both quantifiable and nonquantifiable aspects of change (Bergh \& Pattinson, 2003). Research and methodological findings from the study of KMC within NICUs in New Zealand is urgently required to add to the body of knowledge around this important topic.

Lastly, reviews of the social construction of NICU care and institutional racism in New Zealand were conducted due to their relevance to the topic of this research. Through a constructivist lens, the contributions of personal, organisational and societal 'culture', including wider socioeconomic factors and normative paradigms of care, are all highly impactful within the social 'world' of the NICU. The biomedical paradigm remains the most informing 'philosophy' within the NICU, the care practices of which leave scant space for true integration of family-centred developmental care. Through their narratives, parents have expressed the desire for increased partnership and culturally relevant care through balancing of medical understandings with biopsychosocial and spiritual knowledges. In Aotearoa, New Zealand, while indigenous voices from the NICU are absent from the literature, it is reasonable to assume from knowledge about institutional healthcare racism within this country that it is a factor in diminishing the experience of Māori in this context.

\subsection{POSITIONING OF THIS THESIS}

This thesis describes primary research conducted in collaboration with NICU community members from one tertiary unit, for the purpose of upscaling their KMC programme. Knowledge about KMC application in the New Zealand context is absent in the literature, as is information about the social construction of NICU care in Aotearoa. In addition, there is no current evidence for quality improvement methods for KMC programme development in this context. This social science research project was designed to provide findings specific to one NICU about their own KMC programme. In addition, it sought to add knowledge to the field of implementation science for quality improvement of evidence-based KMC within the 
complex social environment of the NICUs in the high-income setting. Some findings may be generalisable to other contexts. Next, chapter three describes theoretical and applied perspectives of the participatory action research approach for the purposes of this study. 


\section{SECTION A}

\section{CHAPTER THREE: METHODOLOGY}

The evidence for the implementation of Kangaroo Mother Care with hospitalised babies and mothers in the immediate newborn period is compelling. This is especially important for the babies who require neonatal intensive care. Attempts to implement KMC into clinical sites globally have had variable results, some of the most resistant being NICUs within high income countries. NICU environments are highly complex socio-political settings which may benefit from innovative research approaches which integrate bottom-up and top-down approaches for the purpose of practice transformation. In order to support KMC quality improvement in one NICU, I offered my services as a researcher. It soon became apparent that there were several research-based activities that could be supported and that working alongside the staff in a collaborative manner would provide the best opportunity for sustained KMC change. It was finding an overarching methodological approach in support of organisational KMC quality improvement which was the challenge. Participatory action research (PAR) stood out as an ideal approach.

This chapter describes PAR and details its relevance both to the topic and to my own worldview. It describes how it was utilised and how it unfolded. Five phases of the project were undertaken: a KMC audit, staff interviews, KMC observation and two narrative interviews; one parental and one with the sole enduring staff member involved with the research. It also looks at how the study evolved as co-participants fell along the way and projects were hampered by multiple factors. It then describes my responses to the challenges inherent within the research that threatened to derail the project, including my analysis of the overall levels of participation from within the NICU community. I detail my rationale for adopting a secondary discourse analysis to inquire into the power relations within the NICU space. And why feminist and Foucauldian lenses were utilised to analyse the data further, in order to make sense of what was occurring. Furthermore, additional local and global 
literature relating to the NICU-experience of women and parents were reviewed, and the findings included to ensure that parental voices were adequately represented.

\subsection{PARTICIPATORY ACTION RESEARCH}

Participatory action research is an approach to scientific inquiry which uses collective community investigation to understand the reality of social phenomena through cycles of exploration, action and evaluation (Kelly, 2005; Kidd \& Kral, 2005; Klocker, 2015). One overarching aim is to involve, empower and enhance the lives of the group involved (Koch, Selim, \& Kralik, 2002; Maguire, 1987). PAR has therefore been referred to as one of a group of "liberatory research methods" (Barbera, 2008, p. 142), due to its inherent impetus to democratise knowledge.

The earliest use of the PAR orientation was in oppressed and marginalised groups of Third World and International (non-United States of America) countries, where disparities in many social and economic circumstances were found (Maguire, 1987). More recently, the PAR approach has gained popularity for the investigation of inequities and challenges within the healthcare environment for the purpose of quality improvement of practice and enhanced service delivery to patients/consumers (Foster \& Young, 2015; Khanlou \& Peter, 2005). The use of PAR was especially appealing to me for this research, because I was interested in facilitating a quality improvement project which considered the perspectives of all members of the NICU community involved with KMC. It was possible that the evidence-based practice of KMC was suboptimally implemented within the community which I approached and that the group had the capacity to improve that situation through their own collective resources. Within the institutionalised and hierarchical framework of the NICU, my intention was to use PAR as a methodology to 'hold space' for the lived experience of babies, parents and staff as it related to KMC. This was especially so for any individual whose perspective was marginalised within the NICU environment. A distinguishing feature of the PAR approach is that the "researched become the researchers” (Baum, MacDougall, \& Smith, 2006, p. 854), or alternatively said, research is done 'with' rather than 'on' people (Maguire, 1987). Its distinctiveness from more conventional research approaches lies not in its methods and techniques, but in a shift in paradigm, from a focus on participants as subjects, to participants as partners in the process (Baum et al., 2006). In contrast to other more traditional 
research frameworks, the possibility for academics or 'outside researchers' to become facilitators in problem-solving, providing support, developing relationships and presenting as 'change agents', where appropriate, exists with PAR (Dogherty, Harrison, Graham, Vandyk, \& Keeping-Burke, 2013; Robertson, 2009).

The scope to be able to contribute as a co-participant is an important ideal for me to foreground, due to the nature of my 'outsider' status as a researcher within the unit. The potential for democratising knowledge with PAR methodology provided me with an opportunity to input my own knowledge as a ex-NICU parent and KMC researcher. In support of PAR inquiry for this study, I position the NICU as a complex and highly dynamic socio-political environment, occupied by people with differing perspectives and many versions of 'truth'. Participants were encouraged to develop attributes of deep respect for the perspectives of all members of the community, myself included. As well, a belief in our capacity to make self-directed change with the possibility for a shift in paradigm to a non-normative research approach, was considered supportive. I was interested in whether these PAR premises would be tolerated by the group I was proposing to work with, given that the most common research approach within the biomedical environment was likely to be of the positivist, scientific tradition.

Classical PAR is operationalised through an iterative plan-action-reflection process, with each new phase building on knowledge from the previous one. Pictorially, PAR projects are often shown as an iterative spiral with at least three cycles of planning, activity and reflection, represented in figure 3. 
Figure 3: A Simple Representation of PAR

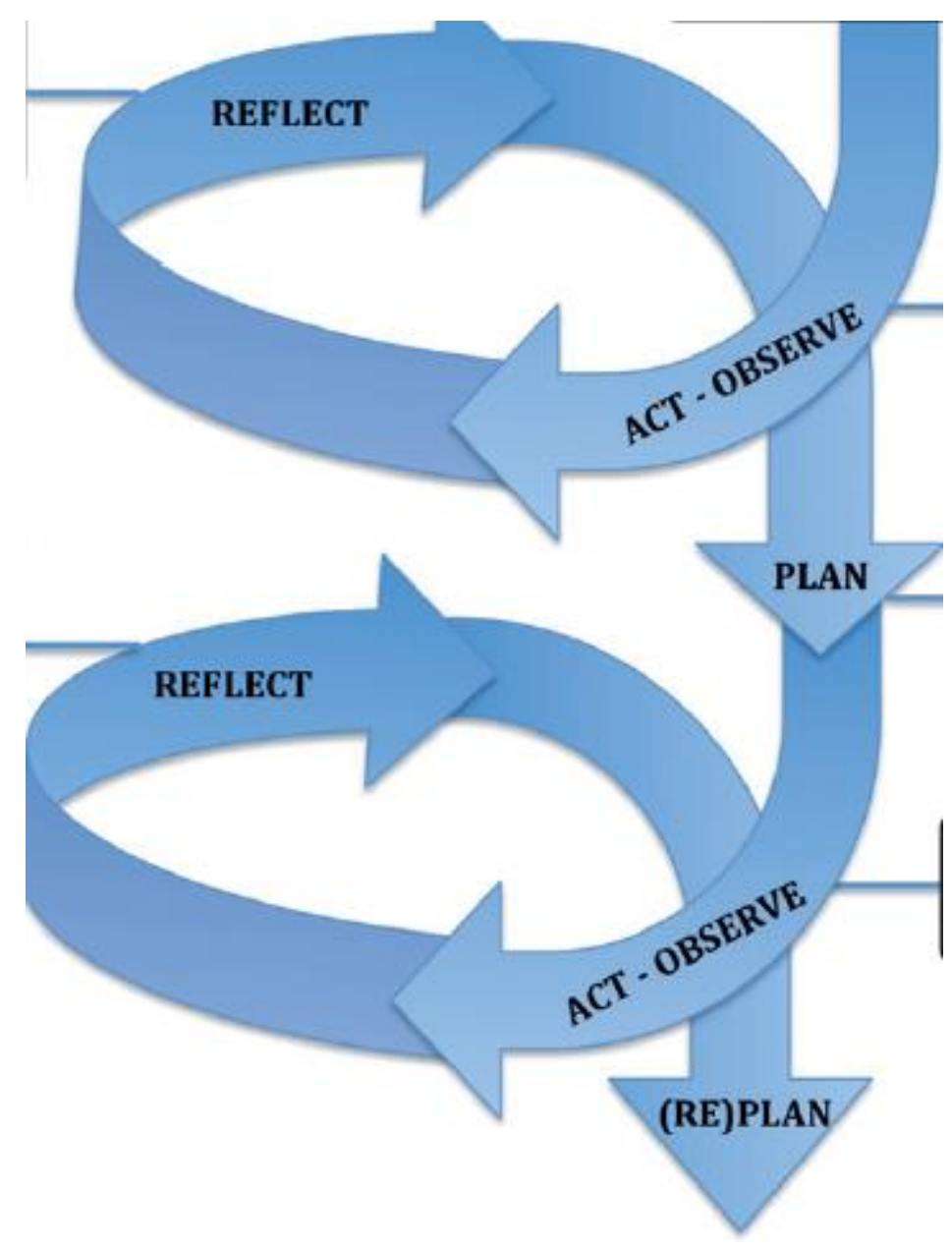

Adapted from "Scientific rigour and innovations in participatory action research investigating workplace learning in continuing interprofessional education" by S. Langlois, J. Goudreau, and L. Lalonde, 2014, Journal of Interprofessional Care, Volume 28, p.227. Copyright 2018 Taylor \& Francis. Adapted with permission. 
Three main characteristics set PAR apart from other methodologies (Baum et al., 2006):

i. The purpose is to educate and enable action, through ongoing iterative cycles of planning, activity and reflection;

ii. An intention to share power between all participants, both researched and researchers. Coined by Meyer, (2000, p. 178) as "the democratic impulse", resulting in liberation of the group; and

iii. Advocacy for the active participation and empowerment of people whom the research involves and will affect.

These characteristics are underpinned by the following principles:

i. Critical consciousness is developed through the pairing of action and reflection to increase self-criticality of each individual (Kidd \& Kral, 2005);

ii. Power relationships are addressed through participation and commitment to democratic principles assuming the "fundamental right of persons to contribute to decisions which affect them" (Reason, 1998, p. 3); and

iii. Cycles of planning, action and reflection are conducted in support of the achievement of change and transformation (Baum et al., 2006).

\subsubsection{Philosophical underpinnings of PAR}

Participatory action research inquiry is located within the constructivist-interpretivist research paradigms (hereafter, simply called constructivist), anchored within the social sciences (Ponterotto, 2005). At the heart of the constructivist paradigm is the ontological position that the nature of reality or 'truth' is created in the mind of each individual and influenced at the researcher-researched interface, with the possibility for emergence of a new and co-constructed 'reality' (Ponterotto, 2005). In the realm of constructionism, 'reality'is not an objective phenomenon, indeed, "people invent their reality" (Koch et al., 2002, p. 110). In contrast to the positivist goal to objectify and explain phenomena through isolation of a single, distinct truth (dualism, objectivism), the constructivist goal is to explain social phenomena through the understanding of subjective meaning (Ponterotto, 2005). Simply put, "experience can be a basis of knowing" (Baum et al., 2006, p. 854), influenced by contextual factors such as life experience, social environnment and social interactions. In addition, constructivists maintain that emergence of meaning that lies outside of an 
individual's awareness is made possible through the interaction of the researcher and their object of research, or in other words, "brought to consciousness" (Ponterotto, 2005, p. 129).

The epistemological stance of PAR is the commitment to co-construction of individual and collective reality by 'giving voice' to all stakeholders, departing from traditional research processes by involving participants in as many stages of research as possible (Cahill, Cerecer, \& Bradley, 2010). The values of all participants, including the broader sociocultural context, are considered and inform the epistemology with ethical and political perspectives (Carter \& Little, 2007). With PAR, there is value placed on the inherent questioning of power structures, whereby knowledge is understood to be politicised and inherent in existing societal power constructs (Baum et al., 2006).

In addition, activism is inherent in the PAR process, with the generation of knowledge and reform inextricably linked to community activity (Kidd \& Kral, 2005). Although a lofty version of the 'ideal situation' is not always achieveable, the PAR process may offer personal and collective change through self-awareness, selfknowledge and resulting empowerment. One effect may be the creation of small, yet significant improvements in the lives of people (Koch \& Kralik, 2006).

Whilst the constructivist paradigm aligned strongly with my own worldview, I anticipated that the intersection of PAR methodology with the biomedical paradigm of the NICU was likely to be incongruent. This perspective was based on my understanding that biomedicine is underpinned by traditional, quantitative scientific inquiry and reductionist in nature, resulting in the possiblity for diverging frameworks with little 'shared language'. That said, there is an undoubted movement within healthcare research and scholarly literature toward a more integrated quantitative-qualitative approach for the purpose of knowledge construction and translation (Bate, Mendel, \& Robert, 2008; Brockman, 2015; Clark, 2013; Cruz \& Walt, 2013; Dogherty et al., 2013; Friesen-Storms, Moser, vander Loo, Beurskens, \& Bours, 2014; Khungern, Krairiksh, Taasaniyom, \& Sritanyarat, 2006). The shift is at least partly due to recognition of "barriers to the uptake of the findings of traditional quantitative biomedical research in clinical practice” (Meyer, 2000, p. 178). This new paradigm speaks of translating research from "bench-to-bedside" 
(Goodyear-Smith, 2017, p. 268), requiring participatory inquiry which engages stakeholders and end users in co-design of research within their own context (Barbera, 2008; Goodyear-Smith, 2017).

I theorise that disparities in the quality of maternal-child healthcare delivery within the NICU space exist and are partly due to a lack of women's empowerment within the client-provider relationship, including a lack of acknowledgement of the infants' experience (D'Agata et al., 2018). The concept of evidence-based medicine has been described within the literature as having biases toward patients and carers which may "inadvertently devalue the patient and care agenda" (Greenhalgh, Snow, Ryan, Rees, \& Salisbury, 2015, p. 1) through lack of patient input into research and suppression of patient voice (Greenhalgh et al., 2015). PAR was used for this research to raise consciousness about the inequities of KMC provision for infants and their whānau/families in addition to providing a tool for action-based practice change. Whilst comprehensive implementation of change within the NICU has been acknowledged as "highly difficult... lengthy" (Ballweg, 2001, p. 58), it was in this direction that I pointed the efforts of this research.

\subsubsection{Nuances of PAR participation}

Participatory action research scholars describe three factors involved with 'realworld' research using PAR methodology, relating to: levels of participation (Jacobs, 2010; Gatenby \& Humphries, 2000); positions of participation (Jacobs, 2010); and asymmetry between participants (Penrod, Loeb, Ladonne \& Martin, 2016). There is no 'ideal' when it comes to participation, and feminist PAR researchers have described their multifaceted experiences working with women as co-researchers on projects aimed at democratisation and transformative change (Gatenby and Humphries, 2000, pp. 94-95):

Sometimes we [academics] struggle with the concept of participation... the level of participation varies so much according to the wishes of the participants... participants choose to participate and that they choose the level of participation... Participation also varies a great deal between individual women... Our understanding of that is that the amount of participation must be left to each individual, that this is one way in which participants maintain their own power.

In response to the impetus for evaluating levels of participation and enhancing validity of PAR research, Jacobs (2010) described using the ladder of participation established by Pretty and colleagues (1995), "a tool to trace the development of 
participation" (Jacobs, 2010, p.373). The ladder of Pretty (table 1) details levels of participation from level 1 (the lowest, none) through to level 7 (the highest, selfmobilisation), where the top three rungs are deemed to emphasise the empowering potential of collaboration and collective action (Jacobs, 2006, 2010). I compared the levels of participation in the five phases of this study against the ladder of Pretty, represented later in this chapter (table 2, p. 86).

Jacobs (2006) had this to say on levels of participation measured against the ladder of Pretty (p. 572):

Whether all levels in this figure [ladder of Pretty] should be defined as modes of participation and what levels of participation contribute to empowerment is open to discussion. From the viewpoint of the 'critical thinkers' in this field, participation should be empowering and not just a situation where local people work with a researcher or professional for the latter's convenience. This means that only levels 4 , 5 and 6 [later re-named 5, 6 and 7] can be said to be modes of participation and therefore empowerment.

\section{Table 1: Ladder of Pretty}

\section{Self-mobilization}

6. Interactive participation

5. Functional participation

4. Participation by consultation

3. Participation by information

2. Passive participation

I. No participation
Community members set their own agenda and organize for action. Professionals have a role in the background, are facilitative and supportive but only if asked.

Professionals and community members work as equal partners in defining the problems or needs and the strategies for change. There is a sharing of knowledge and valuing of 'local' or 'lay' knowledge. Professionals facilitate and support the process.

Community members are involved in decision-making and the development and execution of programmes or activities. Professionals are in control and take responsibility for the process.

Community members are asked to give their opinions on the program plans. The professionals decide what to do.

Community members are informed in an early stage about the program plans and are given the opportunity to ask questions.

Professionals are in control of the program; community members are informed about the program.

Community members are not informed about the program, only about the activities for which they have been recruited.

Gratis reuse of Table 1 “The Ladder of Pretty” by Gaby Jacobs, 2010, Action Research, volume 8(4), p. 369. Copyright 2018 by Sage Publishing. Reprinted with permission 
In addition to levels of participation, Jacobs (2010) describes "three different positions in relation to participation" (p. 377) which may create clashes both within and between participants: empowerment, academic quality and practical usefulness (pragmatism). It is important that PAR practitioners are reflexive and identify which position they are occupying at any time, myself recognising that I occupied all three positions over the course of the research project.

The empowerment focus was an ideological position which I occupied for the majority of the KMC project, also identifying this in other participants who seemed most interested in working collaboratively with various members of the community. An empowerment position was also often associated with a 'rub' - requiring that power relationships and organisational constructs obstructing true participation were identified and transformed.

Next, the scholarly position of academic quality was expectedly high from myself with respect to methodological application, and highly identifiable from several of the practitioners involved in some aspects of the research. There was tension associated with academic quality, however, that appeared to be associated with a belief from some participants that 'high quality' research is only derived from the quantitative traditions.

Lastly, the pragmatic position relating to the usefulness of the research and its outcomes was ever-present for participants. Where individuals sensed lack of utility or applicability of the research, often around whether they could see possibility for change (or not), participation would drop off according to a pragmatic approach which focused their time and efforts on activities deemed higher priority. From my standpoint, the large demands on my own time and resources meant that I had a compassionate leaning toward the pragmatic position, acknowledging the necessity for high usefulness of the project at the 'coalface' for high participation. Ultimately, it is important for PAR researchers to understand the constant inter-play between these three positions and how they affect participation, highlighted in this example by Jacobs (2010, p. 380):

When time pressure increased, different priorities regarding the participation of community members in the project were presented. The ideological position, aimed at empowerment, collided with other goals of achieving a product of high academic standard or working towards a practical program, both of which did not necessarily involve a high level of participation. 
The third and final intricacy of PAR worthy of mention here is the potential for asymmetry of power between participants, particularly where a diverse group of stakeholders are coming together for the purpose of research in hierarchical organisations (Penrod et al., 2016). The necessity for identifying and minimising asymmetries for the purpose of participant engagement is an important part of collaboration between 'outsider' researchers and 'insider' participants, described this way by one PAR group (Penrod, et al., 2016, p.145):

Because interactions of researchers and community members across the iterative cycles of PAR are marked by asymmetries, we were acutely sensitive to the asymmetry present when highly-credentialed, doctorally-prepared researchers interacted with insider-staff members who complete high school or some college. Conversely, consider the asymmetry of the prison staff members' superior understanding of the context of corrections care in contrast to that of visiting researcher-experts. Action research focuses on creating engagement opportunities that maximize the unique contributions of individual team members while minimizing asymmetries.

I share the stance of other feminist PAR scholars that research is always political, and it was an unrealistic expectation to make conscious and/or eradicate the inherent and dynamic power asymmetries that were active within the NICU environment (Gatenby \& Humphries, 2000; Gustafson \& Brunger, 2014). To mitigate power imbalances from my own activity within the research process, I practiced high reflexivity and sought to engage in reciprocal communication with other participants regarding the research process, also described by other PAR scholars (Gatenby \& Humphries, 2000, pp. 99, 100):

It is the power to interpret the lives of the women involved, which needs much more careful thought. One way we attempt to make our interpretations trustworthy has been to establish the credibility of our analysis by sending out what we write to participants, inviting their comment and by discussing whenever possible what we are noticing with them.

\subsubsection{PAR and the NICU in the literature}

A brief literature search for the use of PAR within the NICU found just 11 peerreviewed papers between the years 2000 and 2016, in contrast to the approximately 5000 studies of PAR in non-NICU healthcare settings. After exclusion of papers which didn't relate specifically to research of KMC, developmental care or the NICU environment, just six papers were applicable to my own research, one of 
which was based 'locally' in Australasia. The six published papers described PAR approaches to the transformation of various practices including developmental care, family-centred care and NICU 'culture of care'. None of the research used PAR to inquire directly into KMC within the NICU. The diversity of the projects is outside of the discussion here, however one overarching theme emerged from all six projects and that was the conclusion about the success of the PAR methodology for inquiry within the complex environment of the NICU.

Desirable qualities included: inclusivity, "Participatory action research methodology enabled the inclusion of staff to find solutions to design and clinical practice questions” (Broom, Gardner, Kecskes, \& Kildea, 2016, p. 1939); effectiveness for quality improvement, “... PAR is an effective method to improve nursing practice and provide the opportunity for nurses to develop new skills of developmental care" (Daramas, Chontawan, Yenbut, Wittayasooporn, \& Nantachaipan, 2008, p. 92); motivational, "The use of PAR methodology was appropriate... helped motivate the personnel to participate as subjects, and be actively involved with the study" (Hernandez, Rubio-Grillo, \& Lovera, 2016, p. 111); collaborative, “The collaboration ensured the engagement for viable and constructive local changes to be used in designing the concept of the father friendly NICU" (Noergaard, Johannessen, Fenger-Gron, Kofoed, \& Ammentorp, 2016, p. 122); and change-promoting, "A culture of developmental care has been successfully implemented...A participatory action research project was initiated as a mode of practice and enquiry. Action research is an appropriate method for change within a large, diverse and experienced staff of specialised nurses" (Stainton, Prentice, Lindrea, Wise, \& Dando, 2001, pp. 6, 14).

It is noteworthy that all six of the studies were conducted by groups from within the NICUs of interest - facilitator-researchers with 'insider' status (Skene et al., 2015). One scholar concludes that whilst the insider approach is beneficial for "engendering local ownership and responsibility for implementing change... shown to be more effective than externally imposed change" (Skene et al., 2015, p. 665), the presence of 'outsiders', for example, NICU parents, bring alternative forms of knowledge important to inform change planning (Skene et al., 2015). This was heartening for me to read, given that I would be facilitating this research from the position of 'outsider'. In agreement, PAR researchers from another project recommended the 
use of "insider/outsider" researchers for strengthening reflexivity and personal insight (Noergaard et al., 2016, p. 127).

Limitations of the studies reviewed included small sample sizes and contextual factors associated with each NICU which resulted in the possiblitiy for poor transferability and reproducibility to other units (Skene et al., 2015). Loss of personnel and lack of participation resulted in loss of continuity and provided barriers to two of the projects (Hernandez et al., 2016; Noergaard et al., 2016). Lack of participation was highly relevant to this research, so again, it was supportive to know that other PAR researchers had encountered this issue and still managed to complete their projects. Authors noted that PAR had the potential to provide a framework of development for quality improvement in neonatal care (Skene et al., 2015) and was suitable for nursing research of developmental care practice (Daramas et al., 2008; Stainton et al., 2001). I concluded from these studies that the use of PAR within the NICU, whilst groundbreaking, was encouraged by other scholars, additional reason enough to continue with the adoption of its use in this case.

\subsubsection{Positioning of the NICU and PAR}

I have positioned the NICU, for the purposes of this study, as a complex and highly dynamic socio-political system. The critical social justice questions being asked in relation to infant care, the dynamic nature of the healthcare environment and the complexity of the population and the caregiving model, led to my assumption that traditional empirical research methodology was unlikely to generate knowledge that effectively addressed this complexity. Interpretation of the meaning with which people in this NICU attribute to their experience of KMC was paramount for two reasons. Firstly, raising the consciousness of one's own circumstances through selfreflection is known to be empowering and transformative of its own accord (Olshansky et al., 2005). Secondly, change is more likely to be both an improvement and sustainable when the origin of transformation is self-determined and selfgoverned (Koch \& Kralik, 2006).

Here I acknowledge the potential juxtaposition of the constructivist methodology of PAR with the biotechnomedical paradigm of the NICU, a possible source of friction for those community members considering participation. Differences between the hierarchical organisational frameworks of the biomedical tradition and the 
participatory paradigm, in addition to lack of familiarity with PAR, was foreseen as a likely challenge to the NICU members. Specifically, there may be resistance to PAR application due to the conflicting philosophical approaches of power-sharing versus authoritative power modes, a challenge to historical top-down hierarchies (Foster \& Young, 2015; Meleis, 2016).

\subsubsection{Qualities of a PAR researcher}

I realised that the objectives of this project could only be realised by the development of my own deep understanding and genuine adoption of the principles of the PAR approach, particularly its participatory and collaborative nature. In alignment with PAR principles, it was appropriate to make my beliefs (and potential biases) explicit as I situated my inquiry (Meyer, 2000). I had three major assumptions relating to the NICU environment based on my experiences prior to this study. Firstly, participants within this setting were interdependent and influenced by a multitude of individual and collective factors which may or may not be fully understood (for example, healthcare profession values, social determinants of personage, normative societal childcare practices). Secondly, there was a strong likelihood for multiple truths and disparate perspectives and the potential for resulting conflict (for example, between individuals, between professions, between various organisational positions). Lastly, there was a strong propensity for power dynamics at the individual, organisational and societal levels to affect the day to day experiences of individuals working and living in this setting (for example, societal norms of patriarchy, assumed gender roles, economic neoliberal ideology).

As an 'outsider' to the NICU community, I came to accept that although I may have experienced difficulty when receiving healthcare services from this group and had in mind some ideas to 'fix' these issues, it was both undesirable and impossible to imagine that I could effect these changes on my own. Indeed, developing an understanding of the multiple challenges facing this community, from all perspectives, would probably give me the best chance of contributing to sustained KMC quality improvement.

Participatory action research requires a highly reflexive, 'full disclosure' approach by its participants. To this end, in chapter one I introduced three experiences in my background which have contributed to my own knowledge of the social complexity 
of the NICU environment. Firstly, I am mother to two extremely preterm babies who experienced extended NICU stays, and secondly, I completed postgraduate study on Kangaroo Mother Care within this environment prior to commencing my doctorate. Lastly, I had received extensive biomedical training within my veterinary undergraduate degree, and possessed significant knowledge in the area of mammalian reproductive biology. Prior to this doctoral study, then, my observations, experience and research had led to an internal 'working knowledge' consisting of the following assumptions:

i. The biology of the mother-infant dyad is suboptimally supported within the NICU where there is prolonged separation of the pair;

ii. Kangaroo Mother Care is insufficiently embedded in practice when compared to the recommended level of evidence-based care policy;

iii. A family-centred care paradigm is considered best practice for support of $\mathrm{KMC}$, but is inconsistently applied;

iv. An enduring hierarchical organisation model predominates within the NICU environment;

v. Partnership between babies' families and the multi-disciplinary team is variably achieved and interrupted by multiple barriers;

vi. Parents' and babies' voices are marginalised by constructions of biomedical power and knowledge;

vii. Task-orientated approaches compromise family-centred, parent-led approaches to infant care, and predominate within a nurse-led, expert-driven model of care.

In addition to high reflexivity, I was gaining understanding from the literature about the desirable attributes of a PAR researcher, with PAR becoming an increasingly obvious choice for my study. The PAR attributes included, but were not limited to:

i. A willingness to "conscientiously apply PAR ideology" (Kidd \& Kral, 2005, p. 188);

ii. A participatory attitude (Kidd \& Kral, 2005);

iii. Preparedness for a high level of personal involvement, interconnectedness and relationship-building (Kindon \& Elwood, 2009);

iv. The ability to maintain one's own critical perspective whilst immersed in the research process and in the face of ambiguity (Torre \& Ayala, 2009); "be 
open-minded, but know your commitments" (Bruner, 1990, as cited in Kidd \& Kral, 2005, p. 190);

v. The capacity for 'inclusive debate' with a critically open mind (KrumerNevo, 2009);

vi. A willingness to engage with one's own personal struggle with embedded beliefs (Kidd \& Kral, 2005);

vii. A caring and compassionate nature, including being quiet when necessary and being able to 'self-soothe' (Kindon \& Elwood, 2009);

viii. The capacity to tolerate frustration, confusion and not knowing where the research process may lead, including that it may 'fail' (Klocker, 2015);

ix. An understanding of the political complexities of human systems, with a desire to overcome inequality through "transforming the existing social order" (Small, 1995, p. 944);

x. A worldview that orientates toward strengths-based growth and mutual empowerment (Small, 1995).

In response to these ten desirable qualities of a PAR researcher, I have reflected and noted my conscientious and compassionate application of PAR principles, particularly in times of duress, when there was lack of reciprocal engagement (i, ii, vii, ix and x). My levels of personal involvement were, at times, so high that I felt they may compromise the study. This was due to feelings of dissonance around the lack of consistency within the KMC programme, which clashed with my own ideology about how care 'should be' (iii, iv, vi). My capacity for discernment and to remain on-track when faced with other peoples' objectives that were outside the scope of the study grew as the research went on (iv), as did my capacity to cope with the tension inherent in the critical-collaborative 'divide' (v). The frustration inherent in such a chaotic 'research space' was seemingly unmanageable at times, however, the muscles of patience, tolerance and persistence certainly built over the period of the study, helped by my strengths-orientated worldview (viii, ix, x). In summary, I feel as though I was in possession of the majority of these traits before the study began, with the PAR process being instrumental in providing me the opportunity to enhance all of them as the study evolved. 


\subsection{HOW PAR BEGAN}

Participatory action research is based on relationship: "Relationships are an important ingredient in understanding social realities, so they are critical in the [participatory] research process" (Barbera 2008, p. 155). The enactment of this project began and ended with the requirement for participatory relationships which entailed mutuality and reciprocity (Barbara, 2008; Gustafson \& Brunger 2014). Without enduring and functional relationship, PAR could not proceed. From my perspective, there were established relationships on this unit which had begun more than a decade and a half prior to the beginning of this field work. I was known to some of the NICU community prior to the beginning of my doctoral fieldwork due to my son's hospitalisation and also through existing professional networks. This is often the case for PAR researchers who may come from 'within' the community they research, both increasing and alleviating some of the complexities involved in the study outcomes, as described by PAR researchers Beringer \& Fletcher (2011, p. 67):

The single most important indicator of full achievement of outcomes was that the work group members developed mutually supportive and trusting realtionships between themselves and with the facilitator.

A preliminary planning phase (PPP) was instigated prior to the design of this study, aligned with recent recommendations by experienced PAR researchers. Langlois and colleagues (2014) advised (pp. 228, 229):

Before engaging in PAR cyclical process, the conduct of a PPP is recommended... First of all, it entails the academic co-participants reviewing the literature to learn about the different professional challenges that potential non-academic coparticipants are likely to face in their everyday practices. The academics should afterward contact the targeted workplaces in order to better understand their respective culture, and challenges. Before completing recruitment, a meeting between academic and potential non-academic co-participants from various backgrounds is organized to explain the participatory nature and the goals of PAR.

In accordance with these recommendations, a PPP was set up with the support of the first and enduring PAR participant for this study, a long-standing NICU employee with whom I had established a relationship prior to beginning my doctoral work. The initial preliminary planning meeting involved individuals and a network of staff with a special interest in developmental care. I broadly described my research interests (Kangaroo Mother Care and participatory methodology) and engaged with the group about their areas of interest, research they would like to see carried out, and their willingness to be involved with a KMC project. Whilst the topic of quality 
improvement of KMC needed no introduction to the members of this NICU, the concept of PAR needed to be thoroughly and repeatedly introduced and foregrounded. A central priority for the preliminary and planning phases of this project was to make explicit the participatory and inclusive orientation of the inquiry and exactly how that would relate to sharing of power, relationship building and personal responsibility within the group (Khanlou \& Peter, 2005).

The choice to adopt the PAR approach involved a commitment to a non-linear, timeconsuming and often unpredictable mode of social investigation, whereby a "sense of ambiguity" was tolerated by all participants (Torre \& Ayala, 2009, p. 389). The process required an indeterminant period of relationship building within the NICU community before research groups became established and cycles of planning, action and reflection could begin. Reported within the literature and borne out within this study, both ethical and relationship processes were more complex and dynamic than other research approaches and hugely time-consuming. Despite these frustrations, the project initally proceeded with promise with an eagerness expressed by participants to engage with the PAR process. This signalled the beginning of almost two years work with the first PAR group, consisting mainly of the people from the Developmental Care special interest group.

One particular intricacy I can reflect on with regards to facilitating PAR, is the possibility that the affected community will not engage with the facilitating researchers' project ideas. Due to the participatory and interdependent nature of the approach, participants are invited to engage with all stages of planning, activity and reflection of the project. Fortunately for this study, there was mutual interest in a quality improvement project for KMC which set the stage to begin to plan the project. A mutual decision was made for me to perform an audit, comparing case note $\mathrm{KMC}$ documentation with KMC policy guideline, and the ethical application process began. From this point, relationships and PAR projects ebbed and flowed for approximately two years (two thirds of the duration of my doctoral field work). After this time, I became cognisant of the requirement to adjust the design of the research from three cycles of PAR, to just one, full exploratory cycle. 
I began to have a lived experience of the political nature of the NICU environment, coming to an agreement with other PAR scholars who note the highly political imperative of this methodological framework (Reason, 1998, p. 149):

it [participation] affirms the fundamental human right of persons to contribute to decisions which affect them... we are not human without community. Participation is thus fundamental to human flourishing, and is political because, particularly in these times, it requires the exercise of intentional human agency, political action in public and private spheres, to encourage and nurture its development

Whilst inviting necessary participation, PAR researchers are also announcing, either implicitly or explicitly, that 'something needs to change': "Fieldwork is inherently confrontational in that it is purposeful disruption of peoples' lives" (Jenkins, 2015, p. 16). Research findings, therefore, are likely to be contentious and require negotiation of multiple and complex boundaries (Jenkins, 2015). It was important for me to continue to affirm that my version of truth was just one of multiple versions, and that my intention was not to inflict damage, but instead to understand more and assist in constructing an improved version of the status quo (Jenkins, 2015).

During the first half of our research activity I became aware of challenges to full and functional collaborative relationships, potentially affecting participation levels and necessitating a re-envisioning of the research approach. The following narrative describes my experience and reflections as the facilitating researcher, including my rationale for modifications to the original research design of the study. After reflection on the capacity to remain true to PAR, including assessment of the levels of participation across the five phases, the project continued. Additional methods including a synthesis of NICU-narratives from women and parents around the world, as well as a discourse analysis to explore power relations within the NICU, were chosen. These changes enabled the continuation of the study by remaining true to the philosophical and epistemological principles of the PAR approach.

\subsection{REAL-TIME ADJUSTMENT OF PAR}

Multiple sociocultural, political, educational, emotional and physical influences, at the organisational and individual levels, served to collectively impede progress in the early stages of this research. The following factors all contributed to reduced capacity for a forward-flowing three-cycle PAR project, including: difficulties in achieving participant 'buy-in'; the time-intensive nature of PAR; fluctuating group membership; loss of project champions; insufficient infrastructure for 
operationalising action-based change; apprehension about the unfamiliarity of the research process; overloaded work schedules; and myself as 'outsider'. Whilst a positive response and willingness to engage with the research appeared to be present within the preliminary phase of the research, translation of this willingness to sustained engagement for three project cycles was ultimately not achieved.

'Buy-in', the engagement of individuals (particularly champions or key stakeholders) within complex organisations for the purposes of research and practice change, is discussed prolifically within organisational theory literature. Participation in a PAR project requires community involvement from the earliest planning phases which is more than merely 'token' and often opposes existing paradigms of research (Evans, Hanlin, \& Prilleltensky, 2007). Initially, there was a degree of buy-in for this project, experienced as a moderate level of participation by members of the first PAR group. Degrees of relationship fragmentation, however, were a constant companion. Lack of sustained buy-in with this project may have been contributed to by 'handing down' of responsibility from management to front-line practitioners without their explicit consent. The level of individual responsibility and relationship-based interactions required by PAR may have been uncomfortable and/or unwanted by those used to a 'top-down' approach. PAR places heavy focus on the integrity of transparent interactions, which were not always available in this setting, leading to varying levels of participation and halting progression of research cycles (Jacobs, 2010). The demands of PAR on time resources has been consistently highlighted in existing literature and I can confirm that it was a recurring theme for me with this research. Within the complexities of a large healthcare organisation, as time pressure on the research process increased, there were ongoing collisions of practical, professional and academic goals to the point that the participatory nature of the project was, at times, jeopardised (Jacobs, 2010). Very small projects, which appeared 'on paper' to be simple and quick to perform, ended up being stretched over extended periods unpredictable ways. To compensate, the capacity to run several projects in parallel is a recommended strategy, assuming adequate planning for this, as a way to ameliorate the time-intensive factor. The requirement to strategically plan for the 'slow-going' nature of the process is imperative for forward progress of the project, as well as management of individual and group frustration. 
Unpredictable and fluctuating membership of the developmental care working group who were engaged as research participants in the early phases of research was a strong factor in affecting the capacity for meaningful collaboration. Fluctuations in attendance, participation and meeting regularity for the 18 month period while I was involved with the group were notable. In addition, the existing infrastructure required for change implementation to occur, including methods of communication and task-allocation were, at best, 'shaky'. I believe this led to disjointedness, fragmentedness, and waning enthusiasm for the project. Annual leave, competing priorities and lack of clarity about who actually belonged to the group were all challenges to regular and sustained PAR activity with this group. Prior to my arrival as researcher on the NICU, the group had 'fizzled' out, with its reinstatement coinciding with my arrival on the unit. Variable meeting intervals of 2-8 weeks then ensued for approximately 18 months, in which time I was involved with various projects unrelated to my own study. These included providing a literature review for a proposal to change KMC protocol (independent of my own research), creating staff and parent developmental care surveys, and various other activities. These miniprojects were mostly abandoned prior to completion. At the 18 month mark, I ceased to be invited to the meetings, with at least one taking place after my exit from the group. This coincided with one of the personnel (a regular group member and KMC champion) leaving employment for personal reasons.

A significant reason for a polarising effect of the PAR approach is the novelty of this methodology in the NICU environment, a setting where biomedicine and traditional positivist research approaches are more likely bedfellows. More usually, data are collected and measured by a primary research nurse or physician, a process less taxing on the time and social resources of the community, when compared with iterative cycles of PAR. Social science research may still not be embedded in highly medicalised and hierarchical environments such as those seen in the NICU, and therefore may not be as well tolerated by participants, described in this narrative by one PAR researcher (MacDonald, 2012, p. 41):

...researchers may have to prove legitimacy to other, more conventional, researchers who are unused to working with open-ended research designs. One of the most frequent criticisms of PAR is that, from a scientific perspective, it is a 'soft' method of research... those employing a PAR methodology may be challenged by other researchers not familiar with PAR to legitimize their research. 
Despite obvious movement toward a more collaborative care delivery paradigm through multidisciplinary interfaces, my perception is that this hasn't 'trickled down' to the coal-face of grass roots research movements in this environment.

Along with participant unfamiliarity of the methodology, additional resistance to the approach probably came from an understandable lack of willingness to be given more work to do in an already too-busy work day. Given the self-reported hectic and already over-burdened nature of the majority of individuals I encountered in the NICU, it was not surprising that participants were difficult to engage in a commitment to ongoing research cycles. The possibility for research collaboration was often met with responses of arduousness. Apprehension and uncertainty about the process was certainly not limited to the participants from the NICU community. As an inexperienced PAR researcher, I was constantly grappling with my own insecurities and unwillingness to trust in the process of a research approach that, at times, felt cumbersome, chaotic and fraught with complexity. The research environment was set against a backdrop of staff being asked to do more with less, resulting in a pressurised system of 'ambulance at the bottom of the cliff' or 'codered' practice. Although interest in a project to upscale KMC was genuine, there appeared to be little sustained energy to carry out non-urgent projects other than meeting the tasks of the day (R. Bear, field notes, p. 23, 22.3.2016):

...discussion followed on Kangaroo Care... with consensus that the unit is often overwhelmed by staff shortages and low implementation of skin-to-skin care...(however) unanimous agreement exists for the desire to upscale skin-to-skin

I believe the real and/or perceived over-burdened health system was a major influence on the slow and stilted progress of this study.

A somewhat surprising development was the increasing resistance to my offers of input into potential projects that the developmental care group would brainstorm. I became practised at offering to facilitate all manner of KMC upscaling projects, which increasingly fell on deaf ears and were not taken up. The reasons beneath this refusal to accept my research facilitation services remained unclear, but likely had a complex set of barriers of its own. Likely barriers were my 'outsider status', as well as a question over my appropriate qualification to research within the NICU. In the second meeting with potential PAR participants, I noted in my field diary: "there appeared to be tension and concerns... (due to) my qualifications to assist in this 
research project" (R. Bear, field notes, p. 32, 12.4.2016). My awareness into the importance of relationship building had largely stemmed from earlier concerns that I may not be tolerated by the group, as I didn't share a common professional background with them. After all, I wasn't a nurse, a physio, or a midwife (veterinarian didn't seem to count), and my self-proclaimed (and legitimate) title of NICU-mother and 'allied healthcare researcher' didn't seem of interest to wary potential participants. Despite what I would now consider minor overt 'stranger danger', the continued respectful communication between myself and the research group seemed to lessen this effect, although I believe a covert element of this did persist. Although difficult at times, my skills as (outsider) PAR researcher began to develop, acknowledged both by myself and through feedback from those involved. As reinforcement of this, in response to my statement that "I probably got a low response to a questionnaire I disseminated throughout the NICU because I was an outsider", I was met by this emphatic statement by one nurse educator: "Don't worry, I get the same dismal response - and I'm an insider" (R Bear, field notes, p. $59,30.6 .16)$ !

In conclusion, over time, I came to appreciate that there was a 'cycle of engagement' inherent in the PAR process. When engaged, communication characteristics between participants could be described as reciprocal, timely, pro-active and efficient. In these times of engagement which lasted from a few days to 3-4 weeks, projects moved forward smoothly and with reasonable momentum. Following these phases, a period of disengagement occured, characterised by one-way communication, lack of reciprocity, large periods of time between group meetings, negligible or absent feedback and lack of clarity about established projects. With persistence and time, however, a re-engagement phase would occur and momentum on the project would increase once again. It was helpful to be aware of this cycle and ensure that I manage my own frustration with the non-linear nature of the research as reported by other PAR scholars. During phases of disengagement, I developed stronger selfmanagement and leadership capacities by reflecting on my own cycles of engagement. I continued to cultivate attitudinal attributes such as persistence, perseverance and commitment to the group cause, without attempting to manipulate the situation. By repeatedly honouring the PAR-principled promises I had made to the group, I continued to build and maintain relationships and deepen my 
appreciation for the pressures inherent in the workday of the individuals involved. Although the disengagement phases were unnerving and sometimes caused me to question the viability of the research, I came to trust that re-engagement would eventually cycle back in its own way. In the mean time, I learnt to continue momentum by persevering with other aspects of the study that didn't require the direct input of other participants.

At an indeterminant point, somewhere between phases two and three of the first five exploratory phases, I became aware that persistently declining participation by members of the first established group was endangering the qualification of the research methodology as PAR. In addition, dissolution of a second PAR group during the third phase of the KMC observational study called into question what this meant for continuing the project as PAR. Such incongruence between the theoretical and the applied was no longer sitting well with me and so I paused to reflect on whether this project could indeed be heralded as true participatory action research. With all the reflexivity I could muster, I contemplated whether I could continue using PAR methodology and remain compatible and concordant with the approach, albeit with adjusted expectations of its application.

\subsection{EVALUATING PARTICIPATION}

In response to variable participation, a pause in the research process facilitated reflection on whether the study could indeed be continued using the original PAR methodology. Deep reflection upon the the philosophical underpinnings of PAR, support from literature relating to its adaptation in various contexts, and evaluation of participation levels across the phases of the study, were all significant points of consideration. Further, I was required to make more explicit the lenses through which the ongoing PAR process could be supported, in particular those of feminist and Foucauldian-informed theories.

The conclusion to my inquiry into whether PAR would or should continue was ultimately affirmed by two factors. Firstly, comparison of co-researcher participation levels against the ladder of Pretty (table 2) and secondly, the enduring participation and support of two research partners: one parent and one key staff member.

Partnership and collaboration with this pair enabled the ongoing democratisation and co-creation of knowledge with community members affected by this research 
(Krumer-Nevo, 2009). The capacity to remain true to PAR philosophies and principles was strongly arguable and therefore the research continued with renewed vigour.

A brief search of the literature for other researchers' experiences with modification of PAR approaches found some evidence of methodological adaptation. Various terms and phrases were used to describe 'tailoring' of PAR methodology. Some examples included "developed participatory action research process" (EricsonLidman \& Strandberg, 2018, p. 1), "modified Delphi in a PAR framework" (Fletcher \& Marchildon, 2014, p. 7) and "guided by participatory research" (Cappella, Jackson, Bilal, Hamre, \& Soule, 2011, p. 486). Scholarly research commonly cites barriers to PAR implementation which may lead to the necessity for its fluidity and adaptation. For example, barriers such as: fraught boundary negotiations, fixed and entrenched power relations and ineffective relationship-building attempts (Gustafson \& Brunger, 2014), traumatic research topics and participant distress (Klocker, 2015), and paradigmatic battles within hierarchical organisations (Klocker, 2012). The perspective remains, nonetheless, that $\mathrm{PAR}(\mathrm{PhD})$ projects are "inherently do-able" (Klocker, 2012, p. 152), largely because of their capacity to be responsive to complexity.

The classical three-cycle PAR process was adapted to one PAR-informed exploratory cycle, mainly due to the drop off in participation from the original PAR group participants. Stilted progress became a pragmatic limitation to completing three full cycles of activity. The involvement of participants of the initial PAR group, comprised mainly of members of the developmental care special interest group, dwindled at each phase to the point that just one PAR member remained from the preliminary stages through to the end. Once sufficient reflection was applied to the question of continuing the project with or without PAR, it became clear that indeed, the PAR process continued. That is, the intention, attitudes and enactment of PAR principles remained, with ongoing invitation to collaborate, consideration of participant feedback, and reporting of findings back to the group. In addition, PAR activity was reflected in the assessment of participation levels as compared with the ladder of Pretty, shown in table 2 (Jacobs 2010, p. 369). 
Whilst the original design of the project envisaged a single PAR group from start to completion, the unfolding process showed PAR participation that evolved and changed as the study did. Although a 'classic' PAR approach was not realised for this study, the overarching aim continued to be served by the methodology and therefore operationalised for the duration. 
Table 2: Comparison of Participation Levels Against the Ladder of Pretty

\begin{tabular}{|c|c|c|c|c|c|c|c|}
\hline 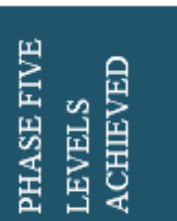 & & & 7 & & & & \\
\hline 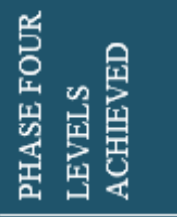 & & 7 & 7 & & & & \\
\hline 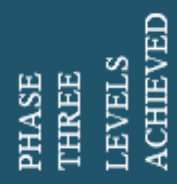 & & 7 & & $>$ & & & \\
\hline 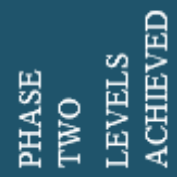 & & & & 7 & & & \\
\hline 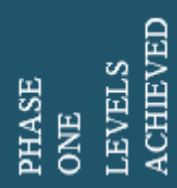 & & & 7 & & & & \\
\hline 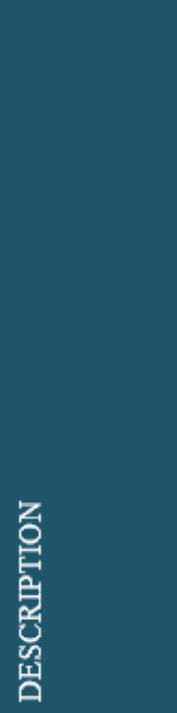 & 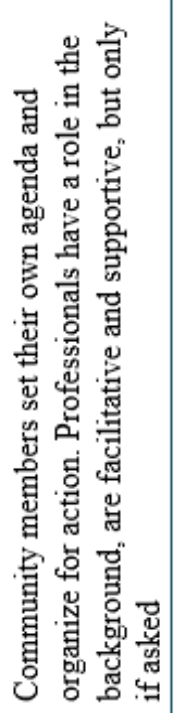 & 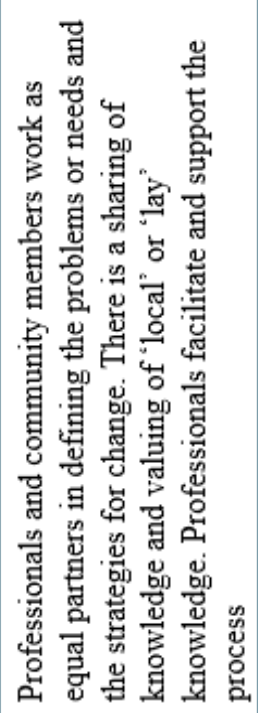 & 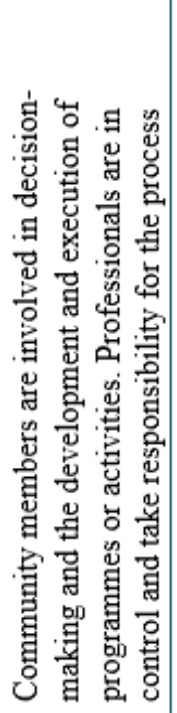 & 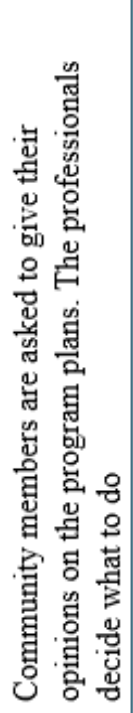 & 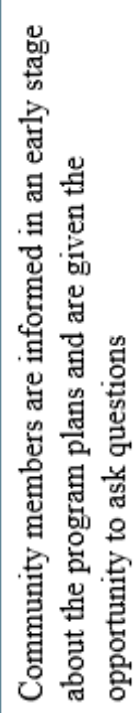 & 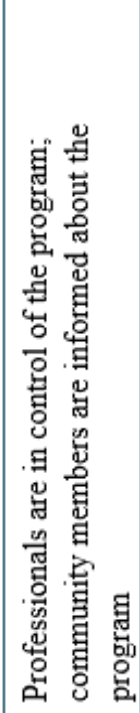 & 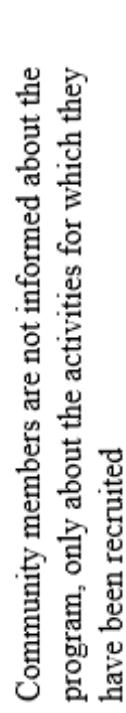 \\
\hline \multirow[t]{2}{*}{ 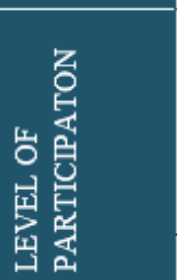 } & 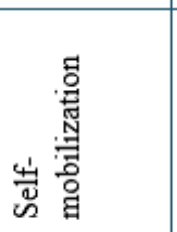 & 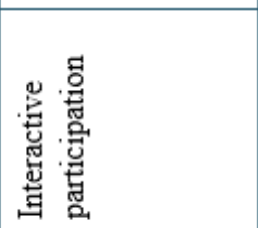 & 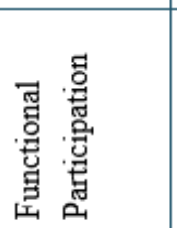 & 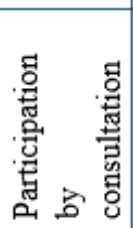 & 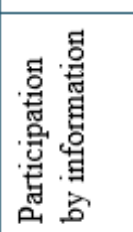 & 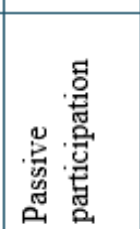 & 苔营 \\
\hline & $r$ & 0 & in & + & $\mathrm{m}$ & N & $\rightarrow$ \\
\hline
\end{tabular}


In summary, it is my participant-supported belief that this research maintained a congruence between methodological theory and its application in practice. It was the fundamental principles of PAR, those of participation, partnership and collaboration for the purpose of action-based social change, that were borne out until the conclusion of the final phases of the study. Accentuating the capacity for adaptation and variation of the methodological approach can ultimately be used to raise consciousness for both the use of PAR in the NICU environment and its ready adaptability in the face of marked complexity. Indeed, I would argue, that social science research involving high community complexity, such as that seen in the NICU, requires the dynamism of a methodological research orientation such as that informed by PAR.

\subsection{GOING DEEPER: FEMINISM AND FOUCAULT}

Feminist critique and Foucauldian theory strongly inform this thesis and have been supportive of ongoing participatory processes throughout the course of the study. Feminist theory and Foucault's perspectives on power, knowledge and discourse are foregrounded because there is a propensity for unequal power relations within the NICU. Biomedicine has been described as hierarchical, medically privileged and technically driven, characteristics which may remain in present-day discourse (Griscti et al., 2017; Meleis, 2016). There may be established discourses whereby authoritative knowledge is held by practitioners in an environment where fear for the life and wellbeing of an infant is prominent. As such, the potential for power differentials that affect the lives of women and their infants within this environment is extraordinarily relevant to research within the NICU (Landzelius, 2006; Roberts, 2008; Spitzer, 2004; Taussig, Hoeyer, \& Helmreich, 2013). Due to the devaluation of a more holistic human experience within the biomedical environment, some argue that power imbalances have resulted in the oppression and neglect of patients and minority discourses (Griscti et al., 2017; Landzelius, 2006; Ventegodt \& Merrick, 2011). Davis-Floyd (2001, p. S5) stated:

despite its pretenses to scientific rigor, the western medical system is less grounded in science than in its wider cultural context; like all health care systems, it embodies the biases and beliefs of the society that created it

There are philosophies common to PAR, feminist and Foucauldian theory. Most notable are the intentions of advocacy and activism through democratisation of 
knowledge and power, with resultant emancipation. Some feminist poststructuralist scholars view Foucauldian theory as supportive of feminist research for examining the effects of institutional power on women, their bodies and mothering (Weedon, 1997; Holmes, 2007; Carabine in Wetherell et al., 2001). Weedon (1997) states (pp. 13-19):

I would argue the appropriateness of poststructuralism to feminist concerns, not as the answer to all feminist questions but as a way of conceptualizing the relationship between language, social institutions and individual consciousness which focuses on how power is exercised and on the possibilities of change... If Foucault's theory of discourse and power can produce in feminist hands an analysis of patriarchal power relations which enables the development of active strategies for change, then it is of little importance whether his own historical analyses fall short of this.

Poststructuralist feminists are generally interested in how bodies are viewed, particularly the tension between essentialist and social constructionist views. They challenge the notion that biology solely determines the nature of women's bodies and focus on how discourse constitutes the body through socially-constructed meanings attributed to it (Holmes 2007). Another feminist scholar proposed a Foucauldian approach by suggesting that "This does not mean we need to treat physical and environmental factors as 'constraints' on social constructs and meanings. Rather, we can adopt an approach which perceives the relationship between physical and social as dialectical, each influencing and informing the other" (Cooper, 1994, p. 440). Foucault (1978) himself stated that "deployments of power are directly connected to the body - to bodies, functions, physiological processes, sensations, and pleasures" (p. 152).

Based on his theory of institutional power relations, Foucault's approach seemed ideal for inquiring into the nature of what remained unseen about KMC on this unit, ultimately enabling the advancement of PAR for the purposes of this research.

\subsubsection{Critical-feminist philosophy and PAR}

Critical theory is a broad scientific paradigm which is closely related to feminist philosophy and informs my own worldview, underpinning aspects of this research. Both critical and feminist theories examine power relations from the ontological perspective of subjective and multiple truths. In addition, critical-ideological theory is underpinned by two basic imperatives: emancipation and transformation.

Ponterotto (2005) described the ontological position of criticalists who (p. 130): 
advocate a reality that is constructed within a social-historical context... conceptualize reality and events within power relations, and they use their research inquiry to help emancipate oppressed groups

I agreed with other PAR researchers who have stated that "The methodological features of PAR are a good fit with the theoretical and practical concerns held by feminist researchers" (Gustafson \& Brunger, p. 999). A critical-feminist worldview is also highly congruent with PAR due to its focus on reflexive practice for the examination of social privilege and constructions (Lykes, 2007, p. 777):

I argue here and elsewhere that reflexivity, particularly the acknowledgement of and critical engagement with "outsider" researcher privilege, is central to such feminist participatory and action research processes.

In my own life, I acknowledge the placement of substantial material and statusrelated privilege with me, due to my position as a middle-class, highly educated, Western woman. I also acknowledge my own experience of the influences of systemic patriarchy in my female life, particularly within the biomedical model, where my strong interest in equality lies. I was very interested in the examination of power relations within the NICU community, in common with other PAR scholars: "Feminist researchers engaged in PAR aim to subvert the power relations that characterize most health research methodologies" (Gustafson \& Brunger, p. 1002). Further congruence between feminist and PAR axiology acknowledges the influences of all individuals involved in the inquiry, affirmed by another researcher who noted: "Democratic, participatory and action-oriented characteristics which seemed to me to be the essence of feminism, I found in PAR" (Krumer-Nevo, 2009, p. 279). To this end, the critical-feminist lens with which I have approached this research, has informed the quality improvement of KMC by advocating for equality of knowledge and power for all members of the NICU community, including infants and their mothers and caregivers. Whilst I 'held space' for the participation and contribution of NICU-mothers with this research, it became apparent that there was little opportunity to include womens' voices within the research setting. In response to gaining the participation of just one woman in the NICU setting of my research, I decided to include further NICU-parent narratives from research within local and global settings, as well as my own story. Findings from the lived experience of women and parents relating to NICU hospitalisation are described in Chapter five. 


\subsubsection{Foucauldian theory and PAR}

The unexamined organisational discursive effects on NICU members and KMC improvement seemed increasingly important to explore through a discourse analysis. This was largely because of ambiguity and discrepancies found between what NICU community members reported was occuring with KMC and what was actually occuring in the programme, a finding that emerged from the primary analyses. In the latter stages of data collection, findings from individual participants did not satisfactorily explain what was actually going on with the practice, most notably its departure from NICU policy guidelines. Larger systemic influences were likely to be in play and yet, as one maternity scholar described, the difficulty in generating legitimate theory around why such a discrepancy existed was akin to "knitting smoke" (Downe, 2008, p. 4). The value of deepening the inquiry into discursive effects on individuals which related to KMC seemed entirely warranted and possible to do whilst upholding the epistemological stance and integrity of the PAR approach (Downe, 2008).

Foucault (1978) theorised that discourses represent bodies of knowledge which are interwoven with power, asserting that "it is in discourse that power and knowledge are joined together" (p. 100). Foucauldian theory is underpinned by the assumption that "knowledge is an outcome of power in that power is important for authorising what counts as knowledge" (Douche, 2007, p. 73). For the purpose of this thesis, discourse is defined as "a belief, practice or knowledge that constructs reality and provides a shared way of understanding the world" (McCloskey, 2008, p. 24).

Discourses are productive, firstly because they create a representation of the topic within a particular historical and sociocultural context; and secondly because they generate power outcomes which work to position individuals within that particular context (Douche, 2007; Edley, 2001). Predominating discourses produce a normative or common-sense notion of 'truth', operating within organisational guidelines, practices and relationships and resulting in power-related social inequalities, often at an unconscious and unexaminied level (Carabine, 2001; McCloskey, 2008). Carabine (2001) was informed by Foucault's concept of normalisation when describing the way in which discourses effectively establish the norm (p. 278):

Foucault has shown that through normalization individuals are compared and differentiated according to a desired norm... normalization establishes the measure by which all are judged and deemed to conform or not. This normalization process 
produces homogeneity through processes of comparison and differentiation. However, we should not think of normalizing judgements as simply about comparing individuals in a binary way... In his notion of 'norm', Foucault did not conceive power as being imposed by one section, class or group of society on another.

As it became obvious that this research would stop after just one PAR cycle, the need for examination of power relations had become more important, leading to my decision to examine available data through discourse analysis informed by Foucault. My awareness of deconstructing dualisms within the NICU, whereby 'non-medical' interventions such as KMC are situated outside of the dominant model of treatment was motivated by the characteristic of feminist theory which resists dualistic thinking. Foucault (1978), theorised that power existed in a matrix that was exerted from multiple loci, asserting that "where there is power, there is resistance" (pp. 9495):

The omnipresence of power... because it is produced from one moment to the next, at every point, or rather in every relation from one point to another. Power is everywhere; not because it embraces everything, but because it comes from everywhere.

Within social science literature, the highly subjective nature of research using PAR has called the trustworthiness of the process into question. It has been noted by some social scientists that "PAR lacks clear scientific criteria to ensure the consistency between the investigators' methodology and philosophy, which jeopardize its credibility" (Langlois, Goudreau, \& Lalonde, 2014, p. 226). In response to this discussion, I feel that it is important to reflect consciously on how the potential shortcomings of this research were addressed for the purpose of enhanced trustworthiness. Credibility of the PAR process used for this study was enhanced by the real-time ability to respond to well known PAR 'pitfalls', including, but not limited to (Langlois et al., 2014): inequity amongst participants within the project; poor participant understanding of the cyclical PAR process; and lack of action-based methods for investigation, rendering the other participants as passive bystanders.

\subsection{TRUSTWORTHINESS AND VALIDITY}

Scientists agree that methodological trustworthiness/rigour is important for all research endeavours. Evaluation of rigour and validity within the qualitative research paradigm were framed from the early 1980s using the concept of trustworthiness, referenced by credibility, transferability, dependability and confirmability. (Davies 
\& Dodd, 2002; Mill \& Ogilvie, 2003; Morse, Barrett, Mayan, Olson, \& Spiers, 2002; Rook, 2017). Viewed through a traditional positivist lens, enduring criticism toward constructivist-interpretivist approaches, with respect to the lack of reliability of research findings and theory-building conclusions, argues a "rigor-relevance gap" (Avenier, 2010, p. 3). Traditional positivist scientific approaches value ideals associated with objectivity, repeatability and measurement of 'hard' data (Chandra \& Shang, 2017). Within the social constructivism paradigm, however, the 'nature of truth' is considered to be subjective, multiple and co-constructed, with findings at least in part due to "the truth as seen by scientists" (Anonymous, 1997, p. 373). The enduring critical perspective is that the quantitative concept of rigour may be partly or wholly inadequate for application to qualitative inquiry (Davies \& Dodd, 2002). Trustworthiness frameworks have since been developed to assist in demonstrating validity and reliability of PAR and other qualitative approaches (Khanlou \& Peter, 2005; Langlois et al., 2014; Morse et al., 2002). The most notable shift for constructing the rigour of a study (and therefore ensuring authenticity of findings) is one of onus of responsibility. Responsibility is transferred from external reviewers (through evaluation after the completion of the study) to resting with investigators by construction of trustworthiness in real time (Morse et al., 2002).

Confidence with the findings and conclusions of this research is based on three tenets of Langlois, Goudreau and Lalonde's (2014) framework for participatory action research. When applied, three guiding principles of empowerment through consciousness raising, collaborative action-stimulus for social transformation, and equity of all co-participants, synergise to enhance the integrity of the PAR approach. Firstly, raising awareness about the beliefs that inform participants' personal, social and professional practices enhances the possibility for self-determined change (Langlois et al., 2014). For this, egalitarian philosophies of equal human worth and status are important. Secondly, the imperative for action-stimulus is important to enhance trustworthiness by ensuring that non-academic participants collaborate as more than passive bystanders in the research (Langlois et al., 2014). Lastly, equity amongst co-participants increases the possibility for genuine collaboration and outcomes which are meaningful for the whole community (Langlois et al., 2014). This PAR 'trustworthiness framework' using equality, consciousness raising and an action-based imperative was initiated at the beginning of our study through a 
preliminary planning phase with potential participants. The first meeting enabled a critical conversation about KMC research within the participants' own setting. The purpose was to foreground the values of mutual agreement and consensus without coercion (Langlois et al., 2014) and to move toward a formal research partnership agreement (Kelly, 2005). As facilitating researcher, I was able to articulate to the group that action cycles were imperative to the study and would be followed by reporting and feedback sessions which encouraged mutual understanding about factors which contributed to the outcomes observed (Kelly, 2005). They, as equal partners in the process, were invited and encouraged to contribute to and participate with the study at every stage. Despite the eventuating variability and inconsistency of participant involvement, processes of planning, action and evaluation/feedback remained possible for all five phases of the study in the spirit of a democratic participatory approach (table 1). In addition to this framework, the characteristics of reflexivity, integration of multiple methods of inquiry and analysis, and the concept of the research audit trail were all involved in enhancing the trustworthiness of the findings.

Reflexivity may be considered the tool by which trustworthiness is upheld in a realtime fashion, because each participant takes responsibility for transparency around his or her own position within the research. Researchers may occupy positions which share experiences with other participants, have no familiarity, or move between the two states, with potential benefits and drawbacks for all positions (Berger, 2015; Jacobs, 2010). It is the capacity of a highly reflexive participant to be aware of their own subjectivities and be able to turn inward to examine their own biases (Berger, 2015, pp. 219-220):

increasingly recognized as a crucial strategy in the process of generating knowledge by means of qualitative research... [researchers] carefully self monitor the impact of their biases, beliefs, and personal experiences on their research; and maintain the balance between the personal and the universal.

It was my observation that the two final and most enduring participants for this research were also the most highly reflexive, communicating their own personal perspectives and experiences with criticality and openness. It is my assertion that high reflexivity is undoubtedly a required trait of a PAR participant-researcher.

Mixed data sources and multiple analytical strategies were applied to exploration of KMC as a means of enhancing trustworthiness (Oen \& Stormark, 2013). This was 
important because of the often contradictory nature between 'what was said, and what was done' with respect to KMC. The purpose of using multiple methods was to describe a more comprehensive picture of the KMC programme from multiple viewpoints (Oen \& Stormark, 2013). This was achieved through interpretation and integration of different sets of data as they weaved, spiralled and touched each other throughout the five separate phases of the study. Firstly, data were collected from different sources (documentation, interviews, KMC observation), at different times and from different individuals within the community. For example, perspectives and meaning attributed to their KMC programme were collected from interviewees representing many different roles and positions within the NICU community. This technique was highly congruent with PAR methodology due to the constructivist ontology of multiple, constructed realities and truths (Baillie, 2015; Ponterotto, 2005). In addition, multiple methods and analyses were used as means to cross validate findings from various phases of the study, glean a deeper understanding of the KMC programme and enhance trustworthiness (Azulai \& Rankin, 2012).

Lastly, trustworthiness was further enhanced using the 'research audit trail', one means of lending strength to the explanations given to the findings of the study. The research audit trail should present enough evidence that the study was "carried out with considerable care" (Carcary, 2009, p. 15) and supports validity, reliability and generalisability (Carcary, 2009). Whilst it was the qualitative notion of transferability, rather than generalisability to populations outside of the research community that was most pertinent to this research, some qualitative researchers argue that the two concepts are strongly inter-related (Carcary, 2009, p. 15):

a researcher can generalise to concepts, theory, specific implications or to rich insights... it is not unreasonable to expect that some findings may be transferable to other orgnaisations... Transferability to other settings depends on the congruence between the "sending context"... and the "receiving contexts"... The researcher needs to provide detailed descriptions of context and phenomena so as to enable others to asssess the findings' transferability.

The detailed explanation of the decision-making and activities of this study are purposeful in revealing the inquiry and its product as it unfolded (Carcary, 2009). In turn, the readers' understanding for trustworthiness of the process, the findings and the conclusions drawn, should be assured. 


\subsection{SUMMARY}

The focus of this chapter has been to provide a characterisation of participatory action research, its underpinning philosophy, and its application as a mode of social investigation within the complex environment of the NICU. Initially, PAR was adopted for exploration of KMC within the NICU space for the purpose of selfdetermined action-based change of the intervention. Whilst it was theoretically plausible to use a classical three-cycle PAR approach within the biomedical space, halting progress required a pragmatic re-evaluation and re-design of the original project and an examination of the validity of the process.

High reflexivity was required to evaluate whether a scaled-back version of PAR, to just one exploratory cycle, could be justified. To rationalise continuing with PAR methodology, an assessment of participation levels with the adoption of additional methods was considered. The use of feminist and Foucauldian lenses allowed me to delve deeper into the power relations within the data of the first five phases of KMC inquiry using discourse analysis. Reflection on the lack of NICU-mother voice emerging from the primary research methods prompted a review of the literature from local and global settings, and the addition of narratives including that of my own NICU-story. Ultimately, the embodiment of PAR processes with the adjunct of feminist and Foucauldian epistemology enabled one exploratory cycle of the project to continue to a satisfactory end. Trustworthiness was enhanced through a PAR framework, ongoing reflexivity, multiple methods of data collection and analyses, and a robust research audit trail.

Section B contains chapters four and five, detailing the PAR processes and methods of the five-phase exploratory cycle of KMC improvement. Chapter 4 describes the methods and findings of research phases 1 to 3, demonstrating the participatory processes for each project, and how the findings informed each subsequent phase. Chapter 5 represents the narratives of people with lived experience in the NICU in three different ways. Firstly, through primary inquiry within this research involving one parent and one staff participant. Secondly, through a review of local and global literature focused on the perspectives of women and parents. And lastly, with the addition of my own NICU-story. 


\section{SECTION B}

\section{CHAPTER FOUR: PAR, PHASES 1-3}

My vision for this project was to engage participants from one NICU with a PAR project for the purpose of quality improvement of their Kangaroo Mother Care programme. The viability of the project was dependent on establishing and maintaining functional partnerships with members of a community who were particularly interested in one aspect of the developmental care practice of hospitalised infants, namely KMC. This evidence-based practice is known to be implemented at less than ideal rates in high-income countries due to many factors associated with parental, whānau/family, staff and institutional challenges (Chan et al., 2017; Chan et al., 2016). The status of KMC within the neonatal units of Aotearoa New Zealand is not currently documented.

The decision to make adaptations to a classical PAR approach reflected the complexity of the environment in which this research took place. Applying PAR within the NICU setting afforded the opportunity to respond to emergent findings with the use of various methods and analyses. As well as the use of multiple methods for inquiry, the research process unfolded in a somewhat unorthodox fashion, largely due to dwindling engagement and participation with the project. In an attempt to simplify the description of a complex and convoluted study, I have sought to describe the PAR process over the five phases of the study in the cyclical pattern that they occured. The findings from each phase, from one through five, informed the subsequent phase and for that reason, I have described the flow of methods, analyses and findings as one unit. Progress into subsequent phases could not be achieved without analysis of each stage, feedback from participants and decision-making based on PAR principles. Although unorthodox to present findings amongst the method descriptions, presenting them this way affords the reader the best opportunity to understand the complexities of the participatory nature of the study.

The project design encompassed five exploratory phases of organisational and stakeholder perspectives relating to their KMC programme. This chapter (Section B, 
chapter four) describes the preliminary planning, participant engagement and design of the study, cultural considerations relevant to Aotearoa, and general ethical considerations relating to PAR. The remainder of the chapter details the first three methods of the research, including: a case note audit of KMC; staff interviews; and an observational study of KMC for pre-discharge infants. The PAR processes for the remaining phases are then in detailed in section B, chapter five. Each of the five research phases are explained separately, including: the specific rationale; aims and objectives; data collection procedures; participant recruitment; research ethics; a description of how these processes unfolded; data analysis and findings; followed lastly by a brief discussion and implications of each phase.

\subsection{STUDY PLANNING AND DESIGN}

\subsubsection{Research setting and pre-project planning}

\section{Choice of setting}

The research setting was a 40-bed tertiary NICU, one of six level three units within the hospital of a major New Zealand city which services a wide geographical area caring for approximately 1000 babies per year (ANZNN, 2018). The reasons for inviting the NICU members of this particular unit were three-fold. Firstly, I had been a consumer for six weeks within this NICU in 2002 with my extremely preterm son, Thomas, and I had a special interest in the functioning of this unit. Secondly, the tertiary environment was of most interest to me, because, through the lens of developmental biology, these babies are likely to endure the highest level of iatrogenic (hospital-based) trauma and therefore be in the greatest need of psychosocial support. Lastly, from a pragmatic perspective, it was closest to home for me, which would provide the best opportunity for extended field work, if required. One senior NICU staff member whom I had previously worked with showed interest in my proposed topic and acted as facilitator of a meeting between myself, my supervisors and a group of interested individuals to discuss the proposed project.

\section{Population details of admissions}

Whilst nationwide figures for the rates and reasons for NICU admission are not currently available, unpublished statistics from the NICU where this study was 
conducted were provided. Statistics included infant and maternal variables for the 2015/2016 period including baby's name, gestation, birthweight, gender, date of birth, date of admission/discharge, and length of stay in the NICU. Maternal date of birth, ethnicity and type of birth were also noted, as well as their home address. A total of 1578 babies were recorded as being admitted to this NICU over the two-year period, with the following demographics relating to prematurity, ethnicity and maternal age noted. Preterm births ( $<37$ weeks gestation) accounted for $50.2 \%$ of NICU admissions. Maternal ethnicity was grouped as 14\% NZ Māori, 48\% Pākeha NZ European and 38\% 'other', where 'other' consisted of women identifying as: Cook Island Māori, Samoan, Fijian, Tongan, Tokelauan, Other Pacific Island, Latin American/Hispanic, Other European, Southeast Asian, Other Asian, Chinese, Indian, African, and Middle Eastern. And finally, maternal age indicators showed 4.4\% of women to be less than 20 years of age, $27.3 \%$ of women were greater than 35 years of age and over two-thirds (68.3\%) between 20 and 35 years of age.

\section{Status of developmental care in the NICU}

Developmental care is an important healthcare framework for the support of KMC, through partnership with parents and understanding of the infants' developmental and biological needs. As I approached the NICU with interest in forming a research partnership, it was important to identify networks or individuals whose focus was mainly on developmental care and associated interventions. On my travels through the unit, it was apparent that developmental care was established in the discourse of the group, visible through posters promoting its implementation, including the recognition of neurodevelopmental cues and developmentally appropriate care. One poster in a staff meeting room alerted me to the presence of a developmental care group, which prompted me to make email contact with the person whose name was associated with it. I made a request to attend these group meetings as a way to network with potential participants for my study, as well as learn more about the functioning of the non-medical and socio-emotional aspects of care in their setting. The spokesperson accepted my request to attend, whilst also highlighting that the group had not been meeting consistently, if at all, over the previous 18 months. It was the intention of the group to begin meeting again at two weekly intervals and I was invited to attend. One of the developmental care group members became a PAR participant for the first phases of the study before leaving employment for personal 
reasons. This employee also had a special interest in KMC and was concurrently conducting a small initiative toward the quality improvement of their KMC programme. I was briefly involved with her efforts through assisting with a literature review involving KMC and a modification of the technique of transfer of the baby from incubator to caregiver.

In addition to the special interest group, there were other indications of the awareness of developmental care, written into philosophy of care documents produced as teaching information for parents, staff and student NICU nurses. Two such documents were available for public viewing on the District Health Board (DHB) website of interest, showing, at least in theory, commitment to a holistic and family-centred nursing care model. In the document produced for student nurses, this was expressed as (Clark, 2017, p. 2):

...we provide culturally appropriate holistic health care for all infants admitted to the Neonatal Intensive Care Unit, while maintaining the integrity and cohesiveness of the family used... practice is based on the belief that each baby is an integral part of his/her family.

The developmental care document produced for parents, and available publicly, states:

...developmental care is used in NICU to describe the plan used to optimise your baby's growth and development outside the womb. Premature babies are born before their brain and muscle development has been completed and need help to cope with their new environment.

\section{Initial engagement of participants}

A preliminary planning phase is recommended to all prospective PAR researchers, which may include "Literature review, visits of targeted workplaces, introduction sessions with interested practitioners, recruitment, identification of the studied phenomenon" (Langlois et al., 2014, p. 227). In the case of this study, preliminary planning commenced with a meeting of five NICU staff members, all with a special interest in developmental care and/or KMC. Potential particpants were invited to attend the meeting by my first point-of-contact in the NICU, a longstanding member of staff with mangagement and leadership responsibilities. My primary and secondary supervisors and I were provided with the opportunity to discuss my interest in KMC research, including my desire to implement a participatory research approach. In addition, my clear intention was to invite the group to collaborate with me on KMC research, for the purpose of upscaling the intervention on their unit, 
based on shared quality improvement efforts from within the PAR group. As the facilitating researcher, it was important to enhance trustworthiness of the PAR process and eventual findings through participation at all phases of research planning and implementation. As per the established PAR literature, this preliminary planning phase is recommended to be undertaken prior to the commencement of three full PAR cycles of planning, action-and-observation and reflection, and did so in the case of this research (Langlois et al., 2014).

The response to my request for participation with this project was initially very encouraging. Discussion amongst the group included what the best 'first step' may be with respect to the suggested research, including the facilitation of a casenote audit. A second meeting was later scheduled with my initial contact once she had had an opportunity to speak with the group privately. In the meantime, a project proposal was provided in writing to the individual with whom I first liaised, a key NICU stakeholder and champion of KMC and developmental care, who ultimately became the only participant who engaged with the study for its entirety. This person held the organisational authority to consent to the study, pending appropriate ethical approval from both the healthcare institution and Victoria University. Of note here is that the first PAR participant committed to the research and remained involved until the conclusion of the study, confirming the beginning of a partnership which was to last for more than two years.

Between July 2015 and July 2017 a research project emerged, the final design of which included five separate phases of the first exploratory cycle within a PAR framework. Cycle one involved five small projects, which engaged various members of the tertiary NICU community, using multiple research methods (figure 1). As introduced in chapter three, it is important to highlight that just one (not three) full cycles of PAR were achieved during the course of this study, the pragmatic reasons for which are multiple and will be described further. It is my considered opinion, supported by the final PAR participant, that the foundation for continuing iterative cycles of research stands for this project, and therefore congruence with PAR methodology remains. 


\subsubsection{Te Tiriti O Waitangi \& cultural considerations}

Te Tiriti O Waitangi (The Treaty of Waitangi, 'Te Tiriti'), 1840, is the founding bicultural document between the first two peoples of Aotearoa New Zealand, the Māori and the European (Kenney, 2011). It is also deeply foundational to my own ethical framework and informs my activity as a researching participant for this study. The principles of partnership, participation and protection upon which Te Tiriti was created informs this study as much more than a token concept imposed upon me through researcher obligations (Victoria University of Wellington, 2009). Rather, Te Tiriti is one lens through which I perceive and respond to the world, a belief system which informs all that I do, and is also integral to the PAR approach. I consider, therefore, that the philosophical frameworks of both Te Tiriti and PAR are highly aligned, inviting genuine partnership, and reflecting the bicultural nature of New Zealand's heritage.

Whilst this research did not focus solely on the indigenous people of Aotearoa New Zealand (Māori-Centred Research), I expected that a proportion of the NICU community and those affected by the research would identify with Māori culture and/or ethnicity. Therefore, in preparation for research, I familiarised myself with the Health Research Council's "Guidelines for Researchers on Health Research Involving Māori” (Health Research Council, 2010), in addition to seeking counsel with colleagues and friends identifying with their Māori community on aspects of the design phase. In doing so, I strengthened my commitment to maintain and enhance mana Māori (Māori authority, jurisdiction, rights) through culturally safe, competent and responsive research founded on true partnership (Farry \& Crowther, 2014).

In particular, the cultural guidelines known as Turanga Kaupapa, established primarily for use by midwives in this country, informed me personally and as a researcher as I worked toward cultural competence with all peoples within the NICU space. Whilst a full description of Turanga Kaupapa principles for cultural competence are outside the scope of this discussion, arguably the most relevant value for those identifying with the Māori culture, is that the realm of pregnancy, birth and childrearing remains one of familial responsibility, which includes the woman and her whānau/family, extended whānau and ancestors (Kenney, 2011). There is marked congruence, therefore, between this major guiding principle of Turanga 
Kaupapa and the foundation of developmental care models for hospitalised infants. That is, the primacy of the infant-woman-whānau/family relationship is held at the centre of all partnership-based care, with effective collaboration based on reciprocity, respect, knowledge-sharing and truly informed consent (Altimier \& Phillips, 2013).

\subsubsection{General ethical considerations for PAR approaches}

My personal ethics and those of Te Tiriti are aligned with PAR philosophy, that requires ethical considerations to be centred on outcomes of group liberation, participation, social justice and empowerment, in line with the methodological ideology. Various frameworks have arisen to evaluate the ethics of PAR research studies within the healthcare arena, the focus of which consider: participant involvement; welfare and autonomy; nature of the research question and outcomes; purpose of the study; aspects of methodological process and context; and opportunities for full participation in the topic of interest (Khanlou \& Peter, 2005). The following recommendations are made to existing research ethics boards in support of PAR studies (Khanlou \& Peter, 2005), i.e. members of a study

i. Are required to have knowledge of a range of research approaches including positivistic and naturalistic philosophies and processes;

ii. Should be familiar with various ideologies, discourse and proposals and therefore be able to recognise whether or not the nature of the inquiry is aligned with the study;

iii. Use alternative frameworks to evaluate PAR proposals which are dynamic and alternate between overall (big picture) ethical concerns and specifics;

iv. Should focus on context-specific ethical considerations, preferably involving the communities participating in the research; and

v. Assess the appropriateness of PAR methodology in answering research questions and suggest other inquiries when suitable.

In addition to the above framework, committees and participants involved with ethical considerations of PAR projects may consider the 'big picture' perspective that ethics forms one aspect of validity and trustworthiness of the research through the following principles: verification of the social and scientific value and usefulness of the research to the participating community (Khanlou \& Peter, 2005; Morse et al., 2002); accountability of the participants to ethical principles through consciousness- 
raising and reflexive process (Davies \& Dodd, 2002; Kidd \& Kral, 2005); and foregrounding of the true emancipatory potential for action-based PAR methodology within the setting of interest (Barbera, 2008; Khanlou \& Peter, 2005; Langlois et al., 2014).

Ethical considerations associated with PAR methodology may be as complex and challenging as the implementation of the approach itself, and have been increasingly discussed in the PAR-related literature (Goodyear-Smith, 2017; Kelly, 2005; Khanlou \& Peter, 2005; Nugus et al., 2012). Ethical review boards are being asked to familiarise themselves and adapt the processes of ethical evaluation associated with non-traditional and qualitative research methods: “...members of Research Ethics Boards could benefit from an increased understanding of the array of ethical concerns that can arise" (Khanlou \& Peter, 2005, p. 2333). Review boards may be expected to facilitate qualitative methodologies and avoid their process becoming an obstacle to research through the phenomenon of "ethics creep - the increasing jurisdiction of ethics committees over research design" (Newnham, Pincombe, \& McKellar, 2013, p. 121). For the purpose of this discussion, ethical considerations are grouped into two main categories. Firstly, broad ethical considerations of this study are described including the important and relevant topic of gatekeepers and champions. The second group of specific considerations, which arose within the context of each of the five research phases, are then detailed as the processes are described. As the PAR planning cycles began, and project methods were decided in collaboration with the participants, specific ethical considerations and research review committee requirements unfolded and were addressed on a case-by-case basis.

\subsubsection{Ethical considerations, gatekeepers and champions}

Securing gatekeeper support as champions at multiple levels of participant (stakeholder) engagement was ultimately necessary for the completion of this study. Review of the literature revealed key findings regarding the importance of interdisciplinary champions for effective KMC programmes, as well as the potential for multi-level gatekeepers to act as a barrier to the intervention (Ahern, McKinnon, Bieling, McNeely, \& Langstaff, 2016; Chan et al., 2017; Seidman et al., 2015; Soni et al., 2016). Participatory action researchers who choose to partner with communities in hierarchical and bureaucratic organisations will be knowingly or 
unknowingly negotiating relationships involving power imbalances at the executive, managerial and frontline levels of authority (Nugus et al., 2012).

For this study, champions who enabled the research were identified in staff positions of NICU leadership, management, education, allied health and frontline medicine and nursing. In addition, I described how a champion within the organisation research committee office, who became familiar with the study due to multiple ethical submissions, was a significant enabler of the project ( $R$. Bear, field notes, $p$. $109 ; 23.2 .17)$ :

I consider (name of the research committee member) to be a PAR participant, of sorts, and would recommend having somebody on the research committee involved in the collaboration (study) due to the complexity of the process.

I also experienced obstructive gatekeeping at the level of NICU leadership, management, education and allied health. In addition, the research was heavily affected by a void that was created when a key facilitating champion ceased to be involved with the project, resulting in an abrupt halt to the phase that was in process at the time. Although the gatekeeper/champion effect is rooted in multiple factors, I chose to adopt this advice from PAR colleagues who published work about bureaucratic hierarchy and resultant 'managerialism': “to understand participants, action researchers ought to see their participants' responses as organizationally positioned, rather than attributing moralistic characteristics or categories to them" (Nugus et al., 2012, p. 1951).

While scant research has focused explicitly on the topic of the ethics and politics of negotiating complex boundaries, problematic researcher accounts at the organisation-research interface are rife (Badger, 2000; Kindon \& Elwood, 2009; Klocker, 2012). Whilst a more in-depth examination of the role of gatekeepers and champions in negotiating power and facilitating PAR progress is outside the scope of this section, I have provided a brief research-based reflection on their influence within certain aspects of this study. It was ultimately in the cultivation of mutually respectful relationships, reflexivity, and open and honest communication which fostered the collaboration required to see the project through to completion (Nugus et al., 2012).

Ethical applications for this project required multiple submissions to the Universtiy Human Ethics Committee (HEC), District Health Board (DHB) research committees 
and Health and Disability ethics committees (HDEC), described more fully in the individual methods sections. Whilst overarching ethical approval allowed for some phases of the study to roll on, the requirement for participant input meant that each application was managed contemporaneously as iterative cycles of planning, implementation and reflection were undertaken. For some phases, amendments or new applications were required to be made to one, two or all of the governing committees. Frustratingly, the rationale and requirements for ethical approval of each new phase of the study were often unclear, inconsistent and possibly politicised. At least two of the six applications were delayed by the semantics of terminology, putting the process into a holding pattern whereby there was lack of clarity about who was responsible for the ultimate institutional sign-off and approval. In practice, this meant that individual organisational managers, clinicians or gatekeepers within the university and organisation had enormous influence, both enabling and blocking the forward progress of the applications. In the case of the first phase KMC audit, full institutional approval took a total of four months, more than the three months that I had planned for completion of the entire audit. Multi-level stoppages including incorrect and/or inadequate authorisation and time-lag between requests and access of clinical notes were significant factors in the delay of the audit process.

In another case, there was a large delay in the processing of the application of the third phase observational study. This was due to lack of clarity about whether the study could be classified as part of the internal quality improvement processes of the DHB (service evaluation vs clinical audit) or deemed as 'research' and requiring an additional ethics proposal. I was asked to put my study proposal forward to three different quality facilitators and managers in various departments of the healthcare organisation, who could be explicit about which particular application would need to be made. Subsequent to this two month-long process, the proposal was then referred to a fourth person, the Operations Manager of the appropriate health services department, deemed to have appropriate authority to progress the application. Two months after the first contact was made with the quality manager in this department, I was no closer to having the information I required to move forward with my application, and I was informed by one study participant that "I have hit a complete road block with the Operations Manager... she is having a 'hissy' that somebody 
outside the DHB would complete an audit. You need to get your ducks in a row" (R. Bear, field notes, pp. 101-102, 2.2.17).

With this, I went back to the Research Office and was informed that "... the only way forward to address NICU management concerns... this would need to be discussed with the child health governance. Can you please email me your paperwork..." (R. Bear, field notes, pp. 109-110, 23.2.17). I emailed paperwork within the hour. In the meantime, in an attempt to find an alternative pathway to the service evaluation/audit one, I submitted an amendment to the original University HEC approval which had been granted for the first cycle of the project. Given that the idea for the observational study had emerged from findings from the first cycle of collaboration with the NICU staff, it seemed plausible that the amendment would be passed as a continuation of the larger $\mathrm{PhD}$ project. The committee decided, however, that prior to their approval of the ethical amendment and "hopefully just as a precaution, you should complete the HDEC (Health and Disability Ethical Committee) scope of review form... can you please do this now and let me know the outcome" (R. Bear, field notes, p. 105, 16.2.17)? Thus, in addition to dual ethical review by the participating organisational and academic institutions, this small observational project, originally expected to be approved under the umbrella of 'in-house' quality assurance, was subject to the third and most detailed of ethical reviews so far - the ministerial committees established by the New Zealand Public Health and Disability Act.

Confirmation from my supervisor began the process of this third and final application, on the basis that the participants are considered to be a vulnerable population within a high-risk setting. Due process was followed with this 88 question online application, and interestingly, within one hour of submission, the application was deemed 'out-of-scope' for HDEC review, given that (appendix 1):

your application describes a quality assurance evaluation of kangaroo mother care in the...[anon] District Health Board Neonatal Intensive Care Unit. The focus of the evaluation will be on the pre-discharge phase of hospitalisation. By conducting this project the outcomes of babies in the pre-discharge phase at [anon] NICU will be improved... [and] HDEC review is not required for it.

In total, ethical approval for phase three of the study took an additional four months to complete. In theory, the potential benefit of this research for this group of healthcare consumers and the professionals who care for them is large, and far 
outweighs any potential risk to their wellbeing. It seems possible that at least two of the phases could have been supported by the existing quality assurance framework of the DHB, given the collaborative nature of the research and the willingness of some $\mathrm{KMC}$ champions to work with an external researcher on the projects. In effect, the cohesion required for this was not present, and the ethical approval process which is actioned for the protection of those being researched, in effect, provided an extra layer of complexity and barrier to quality improvement of the KMC service. My considered opinion is that this was at least partly due to a lack of familiarity of the participating research committees with the PAR approach to inquiry, in addition to a prohibitive and defensive stance.

Like other PAR researchers, I have come to regard PAR methodology as being more than underpinned by ethics; indeed, it is more an embodiment of ethics, that is, "ethics emerge through human interaction...real-world engagement by (PAR) researchers to intervene in participants' lives requires practical ethics" (Nugus et al., 2012, pp. 1946 - 1947). Now, after foregrounding my experience with individual and personality-based barriers to gaining ethical approval, I will describe broad aspects of participant engagement before moving on to the specific methods of the first three phases of the project.

\subsubsection{Participant engagement}

Underpinned by PAR principles, all contributing members of the community of interest form the study population, and are therefore potential participants in quality improvement research. The philosophical rationale remains at the centre: research is about participants, it affects participants, and therefore participants should have equal opportunity to be partners in designing and implementing research which produces self-determined change. In other words research is "carried out with and by local people, rather than on them" (Peters et al., 2013, p. 3). Additionally, through a critical feminist theory lens, characteristics of inclusivity, generativity and restoration infuse the research. There is a commitment to establishing non-exploitive research relationships which balance power inequalities and potentiate liberation through social change (Barbera, 2008; Jenkins, 2015; Krumer-Nevo, 2009).

In general, participant sampling for each of the five phases was achieved through a multimodal combination of networking, convenience, and purposive means, in 
accordance with the overarching objectives of the PAR methodology, discussed case-by-case below (Baillie, 2015). The inclusion criteria were noted as any NICU community member with a perspective about, or an experience of KMC, contingent with the Human Ethical Committee and institutional approval and their (or their parents') informed consent. There were no exclusion criteria explicity identified. Upon reflection, I identified my own position as immovable on this point of inclusivity. This was, in part, due to my own experience as a mother of NICUhospitalised babies and the desire to foreground parental and infant perspectives. It was also due to respect for staff members and the knowledge they possess, as well as the feminist worldview of full inclusivity and respect for the perspectives of 'other truths'. The bi-directional and reciprocal nature of participant involvement meant that open communication was fostered and individuals were welcomed to approach me or other participants about involvement with various phases of the project. The sociocultural, professional and life history characteristics of the participants were desirously heterogeneous, therefore, in an attempt to capture diverse perspectives, although this was ultimately less purposive than convenience (Robinson, 2014).

In the first instance, networking with an existing contact within the leadership of the NICU of interest, established mutual interest in the study. This individual then approached colleagues within her networks, who had a special interest in the topic, for ongoing preliminary discussions. While this small network was forming, various other participant recruitment methods, based on PAR principles, were employed for the five phases, the first three of which are described in the relevant sections below.

\subsubsection{Project proposal and overall design}

After the preliminary planning meeting and prior to the establishment of a PAR group for the first phase of the study, I was asked to provide a project proposal of my suggested research for discussion with one of the participating team leaders. I compiled a proposal for this key stakeholder, my first point of contact for the study, based on aims, objectives and research strategies, including exploration into organisational, management and individual KMC perspectives of the NICU's KMC programme (table 3 ). The details of the proposed phases were left necessarily open, based on an attitude centred on PAR principles rather than specific method details which require participatory input into all phases of the research, including the design 
CHAPTER FOUR: PAR, PHASES 1-3

(Kidd \& Kral, 2005). The proposal was therefore treated as a conceptual overview of the suggested study, rather than a specific plan. 
Table 3 Proposal for KMC Quality Improvement Research

\begin{tabular}{|l|l|l|}
\hline $\begin{array}{l}\text { LEVEL OF } \\
\text { IMPLEMENTATION }\end{array}$ & PROCESSES & AIM OF PROCESS \\
\hline $\begin{array}{l}\text { Onstitutional } \\
\text { Interview key } \\
\text { stakeholders on their } \\
\text { perspectives about factors } \\
\text { which either support or } \\
\text { provide barriers to KMC } \\
\text { practice within their } \\
\text { workplace }\end{array}$ & $\begin{array}{l}\text { Provide a baseline for } \\
\text { designing strategies for } \\
\text { desired change } \\
\text { Use SWOT analysis to } \\
\text { identify strengths and } \\
\text { weaknesses of current system }\end{array}$ \\
\hline $\begin{array}{l}\text { Management and } \\
\text { infrastructure }\end{array}$ & $\begin{array}{l}\text { Assess physical, financial } \\
\text { and procedural barriers to } \\
\text { KMC } \\
\text { For example: space and } \\
\text { physical resource deficits; } \\
\text { documentation and policy } \\
\text { limitations } \\
\text { Identify embedded } \\
\text { support structures that } \\
\text { enable KMC }\end{array}$ & $\begin{array}{l}\text { Identify critical points of } \\
\text { possibility for change } \\
\text { Harness KMC support factors } \\
\text { for potential upscale } \\
\text { Design method for change } \\
\text { using PDSA framework }\end{array}$ \\
$\begin{array}{l}\text { Figure 4) } \\
\text { and 'human' factors }\end{array}$ & $\begin{array}{l}\text { Investigate with the core } \\
\text { group where pivotal } \\
\text { 'human factors' may be } \\
\text { impacting on practice }\end{array}$ & $\begin{array}{l}\text { Identify areas to support } \\
\text { change through training, } \\
\text { education and mentorship } \\
\text { Design method for } \\
\text { change/upscale of KMC } \\
\text { based on PAR methodology } \\
\text { and PDSA framework }\end{array}$ \\
\hline
\end{tabular}


My thinking at the time of this proposal was that the suggested phases would be carried out using an iterative process of three PAR cycles as recommended by scholars for 'classic PAR' studies (Langlois et al., 2014). Planning for PAR projects would usually provide scope for at least three iterations of exploration, implementation and finally evaluation of any changes with respect to the aims; each new planning phase beginning with the end of an evaluation/reflection cycle (Langlois et al., 2014). In the case of this research, it was just the first PAR cycle of exploration which was the focus of my first two years of fieldwork in the NICU (figure 4).

Figure 4: PAR Cycle One, Exploration of KMC

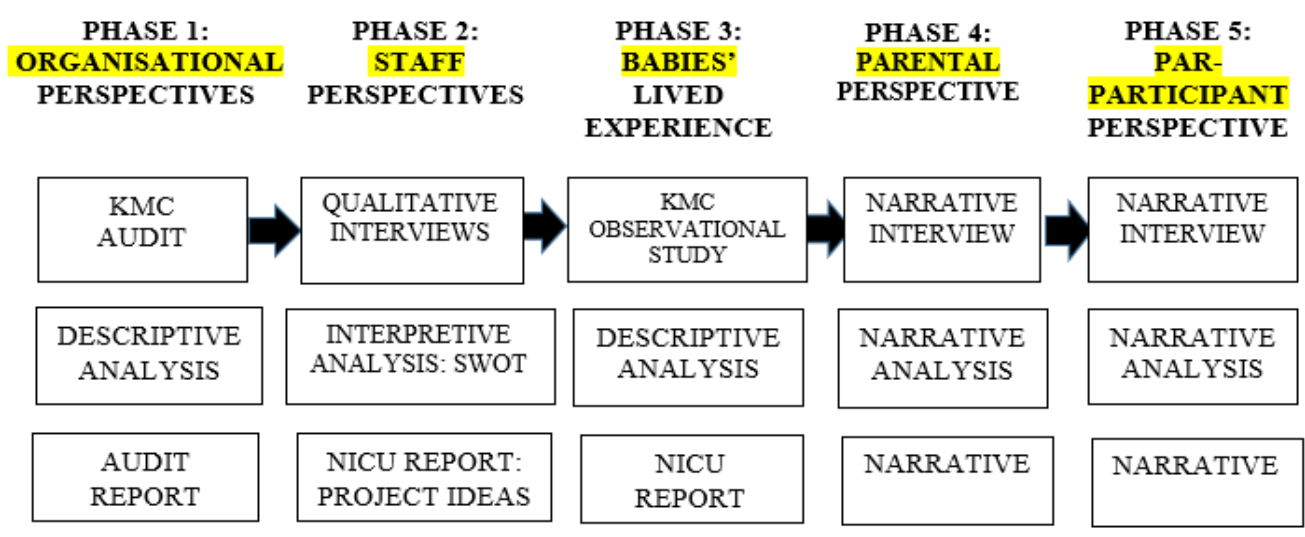

SECONDARY ANALYSES 
The following phrase lends meaning to my experience of the construction of this PAR project: "the reflexive approach makes every PAR project a "custom job" and results in vagueness and ambiguity when the need arises to describe methods..." (Kidd \& Kral, 2005, p. 187). Upon reflection, I concur with this statement, largely due to a need for the research design to remain flexible, fluid, and adaptable to the PAR-requirement for ongoing iterative cycles of participatory planning, implementation and evaluation. It was my conscious intention to construct the details of the research methods in collaboration with the group as the research unfolded, based on a shared philosophical understanding, including:

i. knowledge is shared (everybody is an 'expert' in their own arena);

ii. power differentials are acknowledged and reflected upon;

iii. the raising of critical consciousness is a desired outcome; and iv. collaboration of all community individuals on action-based projects is sought at every level of development.

The PAR ideal of participation in the design phase of the project in this case, however, was not borne out. This was partly due to an absence of meaningful engagement with the process of research design and implementation. It was in the attempt to enact the full three cycles of the project, therefore, that the requirement to depart from a full PAR process arose. It became clear as time went by that three full cycles were not achieveable; the factors of dwindling time, lack of buy-in and everdecreasing active participation by the group being the most notable limitations. In support of commitment to the ongoing PAR process, I contend that the framework for iterative research cycles remains in place and therefore PAR methodology, albeit modified, leaves the methodology in this case appropriately situated for continuation of the research (table 3).

Collaboration with the group of interested staff members whom I met in the preliminary planning constituted the first PAR group, and together we decided on the 'best first step' for exploring KMC within their environment. Following on, new research phases presented themselves based on the findings of the previous phase, including the perspectives of the group involved. Ultimately, several research phases were developed and conducted in parallel, both informed by and informing of the others. Equally important was the engagement with as many of the NICU 
community as participants on the study, for inquiry into transdisciplinary staff perspectives and the parent/baby/whānau unit.

The project structure, therefore, remained open and responsive to the principles and circumstances of the study until the final day of data collection for the first four phases, more than two years after it began. The end of the first four phases signalled a pause-point, seeming an ideal stage to move from the skin-deep findings of the group's KMC programme, to the bones of the less visible factors influencing its application. Findings from the first four phases were analysed and fed back to the NICU participants for their contributions. I became increasingly interested in inquiring more deeply into the data by collaborating with one of the participants, a key NICU roleplayer, who remained engaged and willing to review and comment on the primary findings. This person was also open to revisiting the 'skin of $\mathrm{KMC}^{\prime}$ ' in participation with me, with specific reference to $\mathrm{KMC}$ discourse and the examination of power/knowledge structures, at a deeper level of their KMC programme.

With the ongoing partnership confirmed, a fifth and final study phase was designed to 'close the loop' on the first exploratory PAR cycle. Participation with the single remaining PAR participant, a hugely experienced and important stakeholder for KMC and developmental care within this unit, was crucial to our capacity to finish the study aligned with its PAR-led principles. Our final 90-minute interview captured participant and facilitator perspectives on the findings from the first four study phases, the participant experience of the PAR methodology, as well as participant responses to emergent findings relating to KMC discourse. Ultimately, the research process emerged over the course of the study, with final representation of the exploratory cycle represented in figure 1 (p. 28). 
The PAR approach has scope for multiple methods of data collection, both quantitative and qualitative, the use of which were applied for this work. The inclusion of multiple modes of data collection and analytical methods (not to be confused with mixed method research approaches) were used for this study to strengthen validity of findings and facilitate the achievement of objectives of the study by responding to emerging findings from each subsequent phase. Methods included audit, semi-structured and narrative interviews, and non-participant observation, with the aim of capturing organisational, staff, baby and parent perspectives/experiences of the KMC programme within this NICU. The next three sections provide detailed descriptions of the first three methods. Rationale and aims, participants and ethical considerations, data collection, analysis and findings of each phase are described. Discussion and implications of the findings from each of the first three phases is given prior to a final summation at the end of the chapter, leading onto description of the fourth and fifth phases in chapter five. 


\subsection{PHASE ONE: KMC AUDIT}
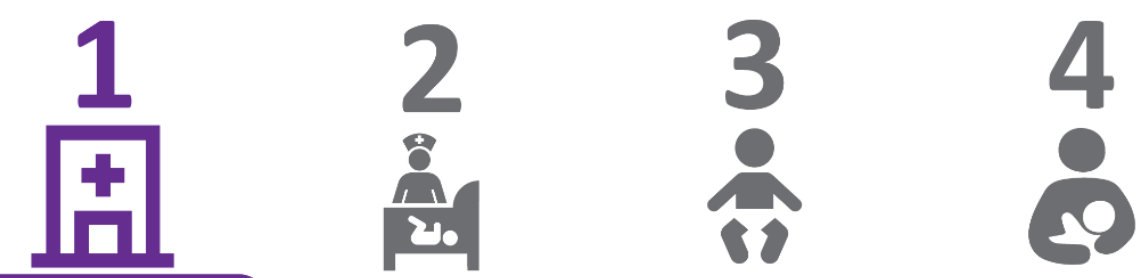

Nov 2015-March2016

KMC Audit

Descriptive analysis

Audit report

\subsubsection{Rationale and Objectives}

Subsequent to the group and team leaders' support for the concept of the research, the first PAR-group meeting led to a collaborative decision for me to conduct a clinical case note audit of documented KMC. The results of the audit, once fed back to the group, were expected to inform an implementation cycle of KMC for the purpose of KMC development. The rationale for the audit was to compare documented KMC practice with the KMC policy guideline. It was hoped that the audit would provide a benchmark of the current functioning of KMC on the unit. The PAR group requested that I compare documentation between one busy and one quiet month, for the possibility of highlighting staff and institutional barriers to KMC implementation. The agreed objectives for the audit were three-fold:

i. to measure implementation rates of KMC for eligible infants;

ii. to compare the documented standard of KMC practice as recommended by policy guideline; and finally;

iii. to compare two months of notes - a 'busier-than-average' month and a 'less-than-average-busy' month.

As the facilitating researcher, I offered to design an audit tool which would record these objectives. This tool was designed to capture aspects of the unit policy on KMC (appendix 2). One member of the PAR group who assisted in orientating me toward the casenotes and acted as my 'go-to' for interaction on this phase, reviewed the audit tool prior to commencement of phase one. 
Once I began the audit, however, I quickly identified that the documentation relating to KMC was not comprehensive enough to capture the depth of information discussed at the first meeting. I was scoping two sets of notes for each baby (observational charts and case notes), and there were large discrepancies between the two documents relating to KMC. Little KMC was documented in most cases, especially details about the timing, duration and caregiver. Quantification of the intervention and its measurement against guideline policy standards was not possible. As a result, the tool was modified for reporting the KMC information which was present. In addition, an adjustment was made to review just one month's notes ( $\mathrm{n}=29$ babies) given that there was too little KMC information within the documentation to attempt a comparison of two months' care. Once analysed, the findings were reported back to the PAR group, both written and verbally, and their feedback on the report captured.

\subsubsection{Participant Engagement and Research Ethics}

From a research ethics standpoint, the KMC clinical audit was included as part of the institution's own quality improvement programme and therefore independent of the requirement for university ethical approval. It was the one and only phase of this study not to require an ethical application for both institutional and university approval. Institutional approval was instigated by registering a clinical audit proposal, supported by two of the PAR participants as well as various clinical and 'quality and risk' managers (appendix 3). The application proposed a retrospective case note audit which compared documented KMC practice with the existing policy standard, conducted for the purpose of informing delivery of best care.

The audit proposal was submitted with the support of one NICU staff member, a known KMC champion on the unit. This participant was very supportive of the project, although they were 'handed' the role by their up-line supervisor, which may imply a measure of duty rather than fully autonomous choice. In addition, this person was soon lost to the PAR group through parental leave, one result of which was a loss of momentum later in the project phases. The audit proposal was submitted in August 2015, and despite being a straight forward standards-based audit application, it took four months to be approved. In addition, due to my status as non-personnel, I was required to comply with three further processes: special staff status; police vet- 
checking; and gaining an external access agreement for the period of data collection, which was completed as per requirements.

\subsubsection{Data Collection}

The adjusted audit tool captured data relating to: infant factors such as age, corrected gestational age and weight; KMC factors such as eligibility, the amount of time in $\mathrm{KMC}$, the caregiver involved and inclusion/exclusion criteria; and any related KMC comments (appendix 2). As stated, whilst staff factors were of interest to the group, details such as staffing levels and acuity were unavailable from the notes. Quantitative data related to babies' gestation, length of hospital stay, KMC eligibility and whether or not they received KMC were captured. In addition, some statistics were gleaned about KMC duration and the providing caregiver. Descriptive findings relating to the use of KMC language, exclusion criteria and insufficient documentation, emerged.

Scrutiny of both the daily observational charts and clinical case notes (two distinct sets of documentation) was made for 29 individual babies who were hospitalised in the month of February, 2015. The process was extremely time consuming, with some individual sets of notes numbering one to two hundred pages, taking six to eight hours to read. In addition to the large volumes of notes, the lack of KMC-related documentation and the possibility of a short sentence being weaved throughout the predominantly medical jargon, made the process extremely slow - somewhat akin to looking for a 'needle in a haystack'. When conferring with the participating PAR member on whether it was deemed necessary to check both documentation sources, the staff member felt it was very important to do so. Her perspective was that the documentation in both sets of notes was likely to be very inconsistent. Sometimes the intervention would be recorded in both sets of documentation; sometimes in one or the either; and sometimes in neither. Examination of both sets of notes therefore continued. At the completion of the review of the first months' notes, consultation with my supervisor resulted in a decision to finish the audit at this stage. I had spent enough time examining the notes to realise that no new information was emerging relating to $\mathrm{KMC}$ and at this stage, the analysis began. 


\subsubsection{Analysis and Findings}

A small amount of data relevant to KMC of 29 babies were gleaned from the audit, reported as simple descriptive statistics relating to eligibility, exclusion, KMC received, caregiver and duration (table 4). In addition, descriptive analysis of three themes which emerged outside of the quantitative statistics added to the findings from the audit.

Table 4: Summary of KMC Audit Statistics

\begin{tabular}{|l|l|l|l|}
\hline & MEDIAN & RANGE & $\%$ \\
\hline GESTATION (weeks) & & & \\
\hline Extremely preterm (<28 weeks) & & & $10 \%$ \\
\hline Very preterm (28-32 weeks) & & & $31 \%$ \\
\hline Moderate to late preterm (33-37 weeks) & & & $59 \%$ \\
\hline & & & \\
\hline LENGTH OF STAY (days) & 20.56 & $0.73-$ & \\
& & 77.61 & \\
\hline KANGAROO MOTHER CARE & & & \\
\hline KMC received when eligible (days) & & & $44 \% ; n=265 / 597$ \\
\hline KMC not received when eligible (days) & & & $56 \% ; n=332 / 597$ \\
\hline KMC exclusions stated & & & $16 \%$ \\
\hline KMC exclusions not stated & & & $84 \%$ \\
\hline KMC caregiver - mother & & & $38 \%$ \\
\hline KMC caregiver - father & & & $20 \%$ \\
\hline KMC caregiver - other or not stated & & & $42 \%$ \\
\hline Average time spent in KMC/day: $<1$ hour & & & $18 \%$ \\
\hline Average time spent in KMC/day: $<2$ hours & & & $22 \%$ \\
\hline Average time spent in KMC/day: $<3$ hours & & & $4 \%$ \\
\hline Average time spent in KMC/day: not stated & & & $56 \%$ \\
\hline
\end{tabular}

The most relevant findings relating to the KMC statistics were: babies who were eligible to receive KMC, yet didn't; whether there was an exclusion criteria stated; who their caregiver was; and the documented time spent in KMC (figure 3, highlighted in yellow). Firstly, of the total number of days that the babies were eligible to receive $\mathrm{KMC}, 56 \%$ of the time there was no documentation of $\mathrm{KMC}$ in the notes. Secondly, for those babies not receiving KMC (or at least, not being recorded as receiving $\mathrm{KMC})$, the vast majority (84\%) did not have exclusion criteria documented. Although it is not possible to say whether babies were actually excluded from KMC when eligible, or that it simply wasn't documented, the assumption could be made that 'if it wasn't written down, it didn't happen'. The majority of feedback from the PAR-group on this point suggests that under- 
reporting, rather than under-implementation, is the reason for $\mathrm{KMC}$ absence in the notes. From the documentation, however, it is not possible to state categorically the truth about this finding. The babies were most likely to be recorded as receiving KMC from their mothers (38\%) than any other caregiver, although it is very likely that this variable was also under-reported. Lastly from the statistics, the duration spent in KMC (for those dyads who KMC was documented for) was not documented $56 \%$ of the time, but where it was, it was most likely to be less than a three hour session per day.

I organised the KMC data which wasn't reducible to a single variable into three main categories, including: significant exclusion criteria; KMC-associated language; and documentation trends. Firstly, whilst documentation of exclusion criteria occurred in only $16 \%$ of cases, half of these cited parental absence or unavailability as the reason: 'no visit by parents today'; 'mum resting' and 'mum late, will try again tomorrow'. Next, almost a third of infants who were noted to be excluded were reported to have medical, physiological and/or respiratory instability and therefore were either implicitly or explicitly excluded from receiving KMC. Other documented exclusions included 'social worker involved', 'declined by father, not ready' and 'getting ready for transfer'.

Secondly, the category of KMC-associated language showed marked inconsistency with the terminology, which led to the grouping of all infants together for the purposes of the audit. The following terms were used and assumed to be all KMC: K/cuddle, Kangaroo cuddles, K.C., skin to skin, prone on mum, cuddled by mum, cares and cuddles, up for cuddle, cuddle, cuddles and/or cuddled, cuddle on lap. There is obvious ambiguity around whether all of these terms represent KMC and therefore lowered assurance about the validity of the quantitative findings of the audit must be assumed.

The last category of documentation trends included: variable and inconsistent KMC documentation both within and between the clinical case notes and observational charts (level 2 and 3); less comprehensive documentation of KMC within night notes and secondary care notes; and clearest documentation for long-stay extremely premature babies who were monitored for extended periods using 12-hourly charts. 
In summary, the most prominent finding from the audit was that it was unable to meet the objectives as set out with the PAR group prior to its commencement. Specifically, KMC documentation was insufficient to reliably measure implementation rates of KMC for eligible infants; to compare documented practice as recommended by policy guideline; or to compare one busy month with one quiet month for the intended identification of organisational barriers to KMC. That said, a small amount of quantitative information was taken from the convenience sample of 29 babies, showing that out of all days that babies were eligible for KMC, just $44 \%$ of babies were documented to receive it.

Despite this, evidence was still able to be brought back to the PAR group, congruent with methodological principles, building knowledge about the group's KMC programme for their own purposes. Participants agreed that while they believed that documented KMC practice did not represent a true picture of actual KMC practice, there was acknowledgement for the requirement of an improved documentation process. Some ideas for enhancement of KMC documentation were brainstormed by members and captured in the second and fifth phases of research.

\subsubsection{Discussion and Implications}

Although the primary aims of the audit could not be met, my reflection after gaining feedback from the PAR group was that the audit was none-the-less worthwhile for providing KMC-related information. Most importantly, the issue of substandard documentation of KMC was highlighted to the group, who agreed with this aspect of audit findings. Participants were unsure, or largely didn't agree with the statistic of infants receiving $\mathrm{KMC}$ on only $44 \%$ of the days that they were eligible, the majority believing that $\mathrm{KMC}$ is under-reported rather than under-implemented. My reflection on the participant perspective of probable under-reporting introduces the following responses. Firstly, I have overestimated KMC by including all 'cuddle-type events' as $\mathrm{KMC}$, which leads to the probability that the $\mathrm{KMC}$ rate is lower than what was gleaned from documentation. Secondly, under-reporting will only be one factor in the low percentage of documented $\mathrm{KMC}$, with a significant proportion of this statistic representing missed opportunities. The low level of reported KMC is an alarming statistic and requires urgent investigation. 
Going forward with a second cycle of PAR implementation could take the approach of re-auditing once improved documentation policy and education has taken place. Brainstorming took place amongst the group about the best way of enhancing documentation of KMC, based on agreement that the current situation is unacceptable. There was also agreement that modification of KMC documentation and its subsequent education to staff may have the dual benefits of raising the profile of the intervention on the unit (upscaling), as well as producing improved documentation.

One condition for conducting the audit was to produce a clinical audit report for feedback to the institutions involved service. This was achieved six months after the end of data collection in accordance with institutional requirements. In addition to mandatory reporting of the audit findings, a separate report was produced for the NICU participants, and presented both digitally and in-person to the group for their information and feedback.

\subsection{PHASE TWO: STAFF INTERVIEWS}
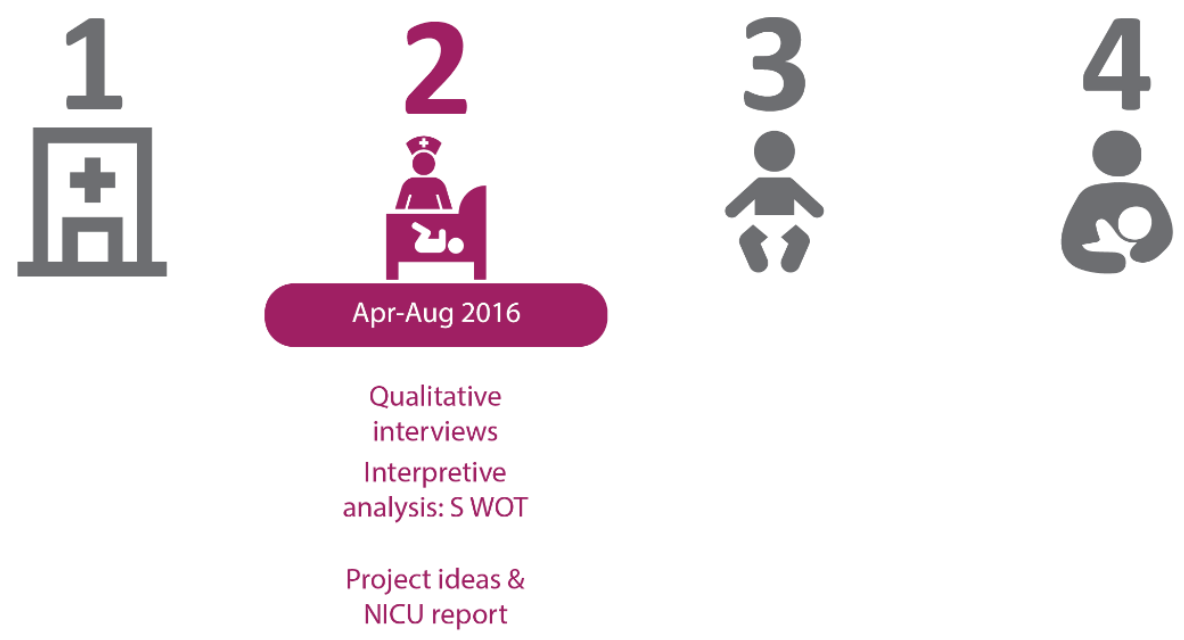

\subsubsection{Rationale and objectives}

Alongside the audit activity, the preparation for invitations to interview key NICU staff members, including nurse managers, neonatal nurses, clinicians and allied healthcare professionals was taking place. The rationale was to engage with the staff in this first exploratory PAR cycle to identify their perspectives about their current KMC programme. I created a semi-structured interview template based on Bergh and 
Pattinson's (2003) publication detailing the development of tools for implementation of KMC. The objectives of the interviews were two-fold:

i. to explore the strengths, weaknesses, opportunities and threats of the KMC programme, from the perspective of the staff; and

ii. to collate ideas for further KMC projects to develop and improve the current KMC service, thereby meeting the 'action' principle of PAR.

The semi-structured nature of the interview method, as well as a request for feedback on the interview template prior to commencement of the second phase, was to encourage group participation in the design of the interview phase. The act of providing an interview template for feedback prior to interview encouraged selfcriticality and focus on the topic of interest. Equally as important, was the embodiment of an action-orientated impetus within the interview, acknowledging that knowledge-gathering alone is not a foundation upon which PAR research is built (Kidd \& Kral, 2005). Rather, it is self-directed problem-solving that is to be emphasised, and to this end, all interviews finished with the question "what, if any, KMC projects would you like to see implemented and is there any way that I could support its facilitation?"

\subsubsection{Participant Engagement and Research Ethics}

Overarching ethical approval for this part of the project was gained through the Human Ethics Committee of Victoria University of Wellington prior to the commencement of data collection (appendix 4). With this, and the healthcare institutions' authority, interviews could proceed with individual consent, following closely the ethical considerations I had noted in the application. Matters of confidentiality and informed consent were of the highest ethical priority for this research phase, given the relatively small numbers of staff, management and leadership-level participants taking part in the interview. Protection of confidentiality was a strong focus, and I took steps to offer participants the opportunity to interview off-site, in case they did not wish to identify their association with me as a KMC researcher. In addition, I took other measures such as the use of pseudonyms and refraining from identifying individuals by their work roles, if this was very likely to compromise their confidentiality (despite the information sheet informing the staff that I may refer to them by their roles). Informed consent was obtained through individual consent forms which were discussed briefly prior to each interview, 
indicating to each individual their full rights within the interview process and for the data produced. They were alerted to the fact that the results may be published in academic journals or presented at conferences, where their confidentiality would remain protected.

\subsubsection{Data Collection}

Invitations to participate in interviews were achieved through convenience sampling via an email invitation to the whole staff list of approximately 160 NICU employees. My primary point of contact in the NICU, by now a PAR participant in the research, agreed to send the research information sheet digitally to each NICU staff member, which included my details. If responded to with an expression of interest, I followed up with a direct email and/or phone call if they provided their phone number. As well, purposive sampling was made through direct contact with each member of the developmental care special interest group with which I had attended meetings, by way of face-to-face invitation and personally providing the information sheet. Further purposive sampling was attempted through invitations to members of professional groups which weren't yet represented for interviews, for example, consultant physicians.

Sixteen participants self-selected to interview, representing practitioners from various disciplines (neonatal nursing, neonatal physicians/specialists, allied health), and from various levels of seniority within the organisation (frontline clinicians, educators, allied support, managerial, organisational leadership). Participants selfidentified with the following roles:

Table 5: Self-identified Roles of Staff Interview Participants

\begin{tabular}{|l|l|}
\hline Practitioner/Educator & 7 \\
\hline Practitioner (Physician, nurse, specialists, allied health) & 3 \\
\hline Practitioner/Management & 2 \\
\hline Leadership/management & 2 \\
\hline Management/educator & 1 \\
\hline Allied support & 1 \\
\hline
\end{tabular}


Interviews took place over a period of three months at the convenience of the participant, at various locations within and outside the hospital. The length of each interview ranged from 30 to 90 minutes. Individual consent was attained prior to the beginning of each interview session. In addition, I gave a brief explanation regarding the nature of the participatory action research, the KMC topic and flexibility of the semi-structured interview process. Semi-structured interviews were based on 11 questions relating to the staff member's role in the NICU, environmental characteristics of their unit, and aspects of KMC including knowledge, education, practice and gaps. In addition, perspectives about quality improvement and change management were of interest, as well as aspects about NICU organisational culture (appendix 7). The participants were highly responsive and reported more than 550 ideas relating to Kangaroo Mother Care in their environment. Interviews were audiorecorded and partially transcribed prior to analysis using a Strengths, Weaknesses, Opportunitities and Threats (SWOT) framework. Partial transcription consisted of full inclusion of all verbal content that was related to $\mathrm{KMC}$, transcribed verbatim (McClellan, MacQueen \& Neidig, 2003). The decision to partially transcribe was also based around pragmatic limitations of researcher time, as well as a lack of necessity to 'go deeper' into the data in the early phases of characterising the KMC programme.

After the final interview I applied for ethical approval amendment to provide each participant with a small donation in the form of a supermarket voucher, to thank them individually for their input (appendix 8). Only one participant rejected the offer of the voucher, based on her perspective that it was not ethical to receive a gift for her participation.

\subsubsection{Analysis and Findings}

I chose to apply a SWOT analysis as a simple way of examining the data, modelling an accessible method of how to produce KMC findings in a fashion that the staff could easily and reliably replicate. Given the iterative nature of PAR, it seemed important to work with simple tools that did not prove too arduous to a group who were already low in time-resource, were they to choose to repeat the cycle at a later date. The SWOT framework is a simple analytical tool which is increasingly being used for purposes of quality improvement in healthcare organisations (Camden, Swaine, Tetreault, \& Bergeron, 2009). Upon reflection, I chose the SWOT analysis 
based on an emerging awareness of the need for pragmatism and simplicity within the research design, in response to the common perspective of staff being overwhelmed in their work. Whilst it is an ideal of PAR that participants be involved with all aspects of the study, I believe I had already subconsciously decided by this stage of the study, possibly with an aspect of paternalism, that I would not burden the participants by inviting them to partake in the analysis of the data. In hindsight I acknowledge that this was outside of the 'PAR attitude' and whilst I continued to invite participation and collaboration in other ways, I would approach the matter of analysis in a different way next time. I do believe, however, that my choice of analytic method had the PAR intention of increased understanding of their KMC programme at heart, therefore providing findings which were highly relevant to the community.

The primary aim of the interviews was to inquire into staff perspectives about their current KMC programme and represent the findings of common supporting and challenging influencers of KMC. A second aim of the interviews was to collate staff ideas for projects to develop and improve the current KMC service. In support of these aims, SWOT categories were determined in combination with subcategories of 'organisational, workplace culture and care paradigm', 'human factors' and 'resources and the environment'. I experienced this as an effective method for capturing a lot of data without being overly reductive. Perceived strengths, weaknesses, opportunities and threats, relating both directly and indirectly to the current KMC programme, are summarised in table 6 , followed by a descriptive analysis including direct quotes from the staff. Individual staff members are identified by pseudonyms to ensure their ongoing anonymity (initials are randomised and do not reflect the actual initials of each person). Lastly, suggestions made by the staff for ongoing KMC quality improvement projects are provided in table 7. 
Table 6: Summary of Themes from SWOT Analysis

\begin{tabular}{|c|c|c|c|c|}
\hline 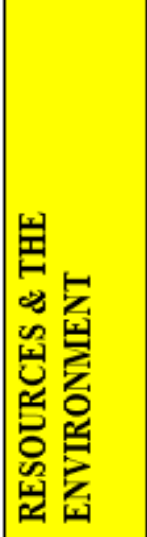 & 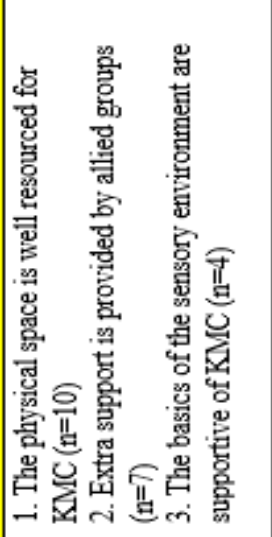 & 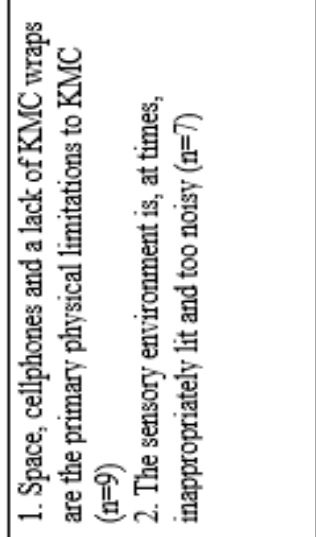 & 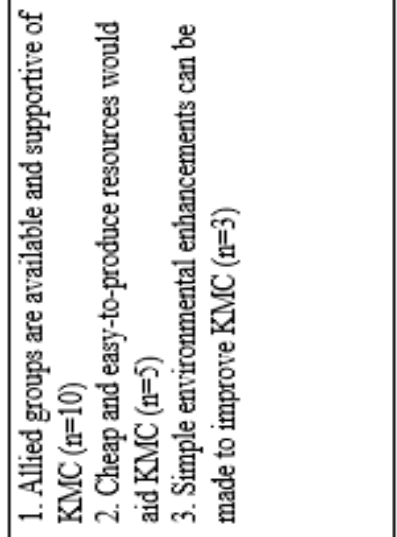 & 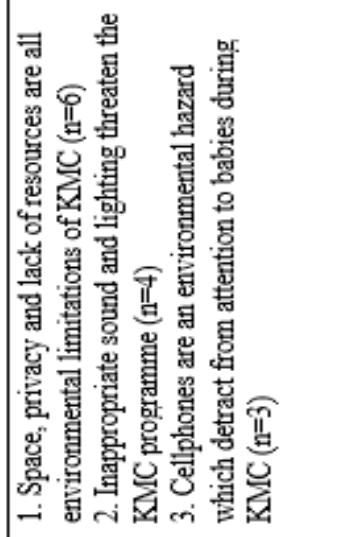 \\
\hline 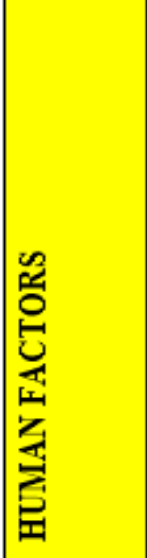 & 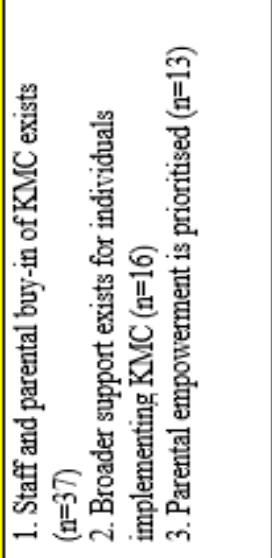 & 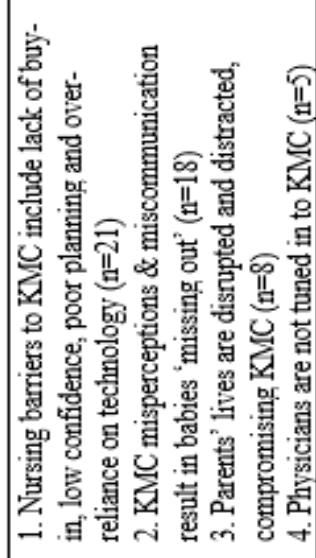 & 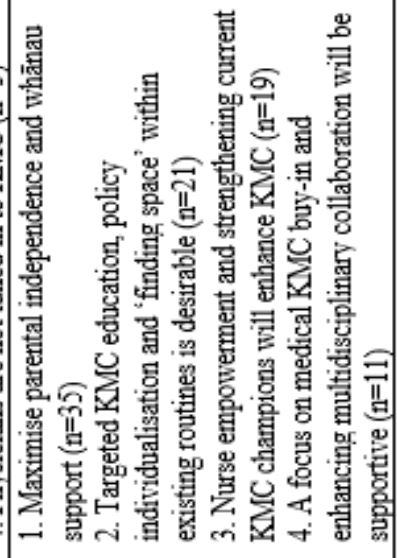 & 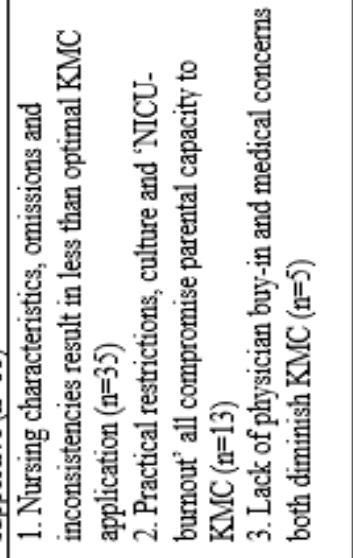 \\
\hline 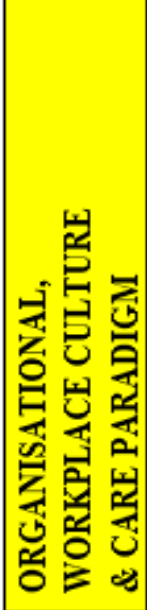 & 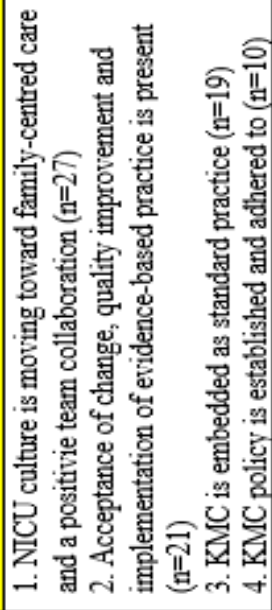 & 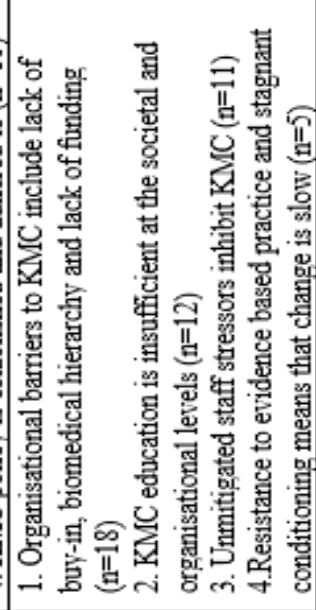 & 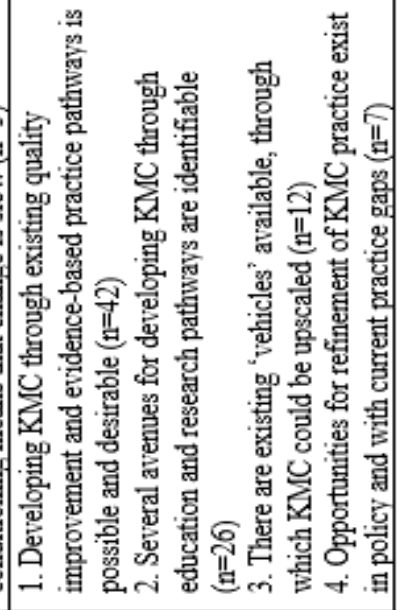 & 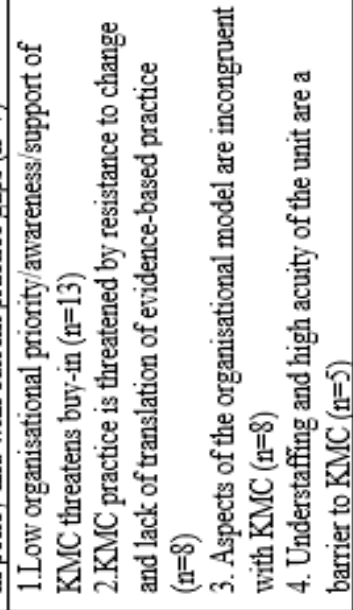 \\
\hline & 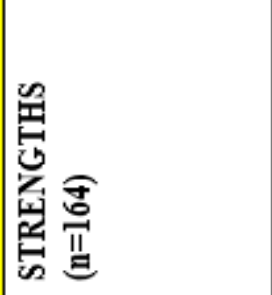 & 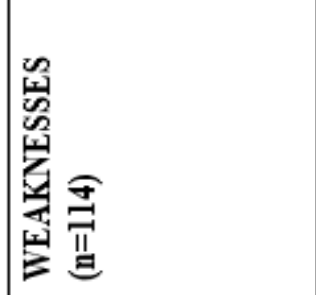 & 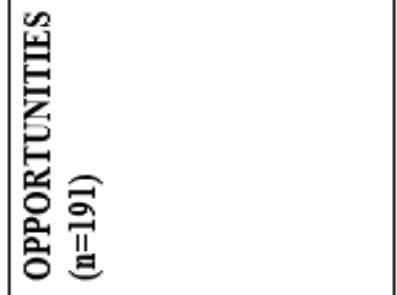 & 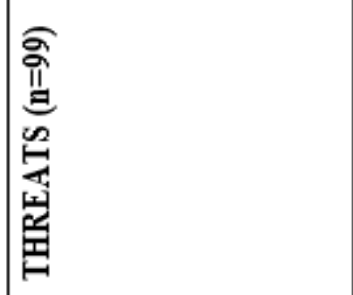 \\
\hline
\end{tabular}




\section{Strengths:}

All 16 participating staff members commented on supportive organisational factors which strengthen the current KMC programme. In particular, the presence of an established quality improvement system, collaborative team interactions and movement toward a family-centred care model, all supported KMC: "we are focused in the direction of doing good for this group of little New Zealanders, and it's incredibly important to realise that change is constant, and to be able to do it in a way that is sustainable" (management and leadership, G.L.).

Some staff also perceived KMC to be embedded as a standard practice, in part due to the existence of both staff and parental buy-in, with the consideration of the wellbeing of the parent often at the centre: "it's almost like standard practice (KMC)" (practitioner and educator, W.W.); "everyone's pretty good at kangaroo care" (practitioner, K.H.). In addition, the importance of skin-to-skin contact for both bonding and breastfeeding was appreciated by some: "I just love seeing how relaxed the parents are [in KMC]... it's just their little baby-box zone" (practitioner and educator, W.M.); "there are parents who do struggle to bond with their baby in this unnatural situation" (allied health, I.O.). Fewer participants (10) expressed that the environment was mainly well resourced for KMC, including sufficient chairs, access to privacy and appropriate space. Four respondents felt that the basics of the sensory environment, such as noise and lighting, accomodated KMC adequately.

\section{Weaknesses:}

The weakest system-related issues noted by the participants were lack of organisational and societal buy-in and education of KMC: "no-one actually teaches the importance of Kangaroo Care to the staff" (manager and practitioner, R.N.A.) and resistance to translation of evidence-based practice and change: "after a while you get exhausted by the system" (practitioner and educator, X.R.). Unmitigated staff stressors such as understaffing and high medical acuity of the unit were also described "realise we are resource-poor... we do not meet best practice minimum hours for nursing staff” (management \& leadership, G.L.).

Many nursing barriers related to KMC were described, especially a lack of buy-in: "nurses need to be able to have time-out without parents being around... it's all lovely to do this Kangaroo Care, but practicality-wise, it may not work today" 
(management, Y.R.). Poor planning by some nurses was noted, "for the most part its (KMC) is encouraged... it can get forgotten unless the parents bring it forward" (practitioner and educator, Z.I.) as was low confidence with some parents: "dads are often doubly as scared (to do KMC), especially if they haven't done cares" (practitioner and educator, W.W.). Resistance by nurses to undressing larger/more developed babies was a common reason given for not enabling KMC, two staff members describing that "nurses don't see the point of stripping down the baby every time [for pre-discharge babies]" (practitioner and management, R.N.A.) and "the baby is dressed now, so the focus is not on skin-to-skin" (allied health, I.O.). Participants also described over-reliance on technology and reluctance to implement $\mathrm{KMC}$ "I still come up against it [resistance to $\mathrm{KMC}$ ]... you've got to go into battle" (practitioner and educator, R.K.). Other KMC-limiting weaknesses included misperceptions and miscommunication about the method: "I think we've lost the plot there... [KMC of larger, dressed babies in cots]" (management and leadership, G.L.); parental unavailability and a lack of buy-in from physicians: "the medical perspective doesn't care so much about that [KMC], it's the 'non-medical' side of things" (practitioner \& educator, Z.I.).

Lack or inappropriate use of space, unavailability of a KMC 'wrap' (to enhance security and privacy), and cellphones as an environmental hazard were the most often reported resource-based KMC limitations: "I don't think parents should have them [cellphones] while they're holding their babies" (practitioner and educator, Z.I.). Almost twice as many responses noted the sensory environment as a weakness (rather than a strength), especially with respect to inappropriate lighting and noise levels.

\section{Opportunities:}

Almost 200 responses were made by all 16 participants on the topic of opportunities for support of the KMC programme, either directly or indirectly. At the systems level, development of KMC through existing quality improvement, education and research pathways were identified: "If we teach the parents well, it's [KMC] actually a load off (the nurses)" (practitioner and educator, Z.I.). The desirability of a shift of infant-care model from 'nurse-led' to 'parent-led' when developmentally appropriate, was identified: "Hopefully we get 75/25 acceptance (to a change in KMC standing transfer), and not the other way around" (practitioner and educator, 
W.W.). As well, existing 'vehicles' could be used to develop and upscale KMC, such as medical rounds, shared study days, staff orientation sessions, developmental care group meetings and the breastfeeding peer support programme.

Parental and nursing empowerment were deemed as important opportunities for enhancing KMC through both education and mentor-type support, commented on 54 times by the group: "people need to be taught the rationale behind the practice [KMC]" (practitioner and educator, B.T.); "we really need to address the gap in [KMC] education of the staff" (manager and practitioner, R.N.A.). Specific goals included: increased parental independence of stable and growing babies: "once they're in cots, parents should be doing everything" (practitioner, E.W.); development of KMC champions: "I agree, that if there was one senior person suggesting [the use of $\mathrm{KMC}$ ], it probably would change" (practitioner, R.N.B.); individualisation of KMC policy; and 'finding space' for KMC within existing care routines such as weighing and bath time. In addition, broadening KMC buy-in across the medical disciplines, whilst enhancing existing multidisciplinary collaboration: "nurses are the absolute link between clinicians and parents" (practitioner and educator, X.R.), were both considered to be opportunities for developing KMC.

Opportunities for KMC improvement through increasing the capacity of existing support networks, such as the Neonatal Trust and peer support groups, were raised. Enhancing the availability of simple and cheap resources such as KMC wraps and a dedicated parent information space were also offered: "it's a bit haphazard, the utilisation of handouts and that sort of thing" (manager and educator, Y.J.). One practitioner wished to expand the circle of caregiving for each infant by including wider whānau/family, a practice which had occurred in overseas settings where she had worked: "if parents can't be here, the next best thing is a grandmother or grandfather who can sit here for hours” (practitioner, E.W.).

Opportunities to enhance the environmental space, particularly with regards to noise pollution and restricted use of cellphones were the most commonly identified sensory improvements to be made: “you don't wanna be contaminating your baby with whatever is on your cellphone... encourage them [parents] to leave their cellphones and belongings in this one drawer" (practitioner and educator, W.W.). 


\section{Threats:}

Six participants, all within managerial or allied health roles, responded with 34 perceived threats to the KMC practice across all levels of operation. Firstly, low organisational KMC priority combined with resistance to change and/or evidencebased practice: "nurses are very resistant to change" (manager and practitioner, R.N.A.), were considered to threaten both KMC buy-in and implementation. As well, aspects of the organisational model were deemed non-supportive of KMC: "unfortunately, in this hospital, there is a lot of 'top-down', it is completely hierarchical here... care is episodic... [it is often] in the too hard basket [KMC]" (practitioner and educator, X.R.); as were the common understaffing and high medical acuity of the unit: "we have such a complex environment here. I think everybody who works here is very aware that a misstep from them will have lifelong consequences" (manager \& leadership, G.L.).

Dominating the 'human factor' threats to KMC were nursing-related factors such as practitioner preferences: "If you've got a sick kid in the room, you don't want every other parent there 24/7" (management, Y.R.); work pressure-related omissions: "I don't think it's done well enough on this unit [KMC], I think we're too restrictive... I flout the rules - that they're only allowed up once a day... I don't know where this one-hour [time for KMC] came from, I really don't" (practitioner, E.W.); and failure to implement KMC when appropriate: "it's definitely something we should be doing more often... I don't see it as a norm [KMC]" (practitioner and educator, X.R.). Parental restrictions related to lifestyle, culture and 'NICU-burnout', a factor for both staff and parents, were next most commonly addressed: "the ones that miss out on it $[\mathrm{KMC}]$ are the ones where the parents aren't around" (practitioner and educator, Z.I.); followed by medical related barriers including lack of physician buyin: "I think parents are given mixed messages at times, about when to pick up their babies or not, and when to let them sleep" (practitioner \& educator, allied health, P.Z.).

With regards to environmental and resource threats to KMC, the themes of inadequate space, privacy and KMC aids (wraps), inappropriate sound and lighting and cellphones as hazards/distractions were all noted: "noise is still an environmental issue... some people are just louder than others. In my mind, we still don't have a good environment... not optimal" (practitioner \& educator, L.A.); "it actually should 
be time for you and your baby (re: cellphone disruption)" (practitioner \& educator, L.A.).

The final interview question asked for suggestions for projects which could be implemented within either the first exploratory, or second implementation PAR cycles of KMC quality improvement. It was my intention to offer to facilitate one of the projects, with the chosen project highlighted in table 7. 
Table 7: Summary of Staff Suggestions for KMC Quality Improvement Projects

\begin{tabular}{|c|c|}
\hline $\begin{array}{l}\text { KMC } \\
\text { DEVELOPMENT } \\
\text { AND } \\
\text { IMPLEMENTATION }\end{array}$ & $\begin{array}{l}\text { 1. Develop KMC champions } \\
\text { 2. Mesh KMC with other daily activities: bathing, } \\
\text { weighing and breastfeeding } \\
\text { 3. Develop and increase accessibility of parent information } \\
\text { area } \\
\text { 4. Create a 'competency checklist' to aid in improving } \\
\text { parental confidence } \\
\text { 5. Improve KMC documentation in daily progress notes } \\
\text { 6. Develop KMC wraps } \\
\text { 7. Engage members of multidisciplinary team meetings to } \\
\text { identify KMC barriers }\end{array}$ \\
\hline KMC EDUCATION & $\begin{array}{l}\text { 1. Develop parental KMC education } \\
\text { 2. Education of Room L* parents: KMC at bath-time, } \\
\text { weighing. Move toward more parent-led care } \\
\text { 3. Staff education modules: standing transfer KMC } \\
\text { method and reading babies' neurodevelopmental cues } \\
\text { 4. Continuing education on appropriate cell phone use (x2) } \\
\text { 5. Consider mindfulness classes/provision of a meditation } \\
\text { space } \\
\text { 6. KMC study day: targeted discussion with relevant } \\
\text { current literature } \\
\text { 7. Target dads for increased KMC education } \\
\text { 8. Educate for parent-led KMC of older/stable/dressed } \\
\text { babies in cots }\end{array}$ \\
\hline KMC RESEARCH & $\begin{array}{l}\text { 1. Research what is happening with KMC once people go } \\
\text { home } \\
\text { 2. Survey parental perspectives pre- and post-discharge } \\
\text { (x2) } \\
\text { 3. Research KMC of babies in rooms } \mathrm{X}^{*} \text { and } \mathrm{XXX}^{*} \text { on } \\
\text { this unit, less than } 30 \text { weeks gestation and close to birth } \\
\text { 4. Research KMC of moderately preterm babies on this } \\
\text { unit. What is happening there? } \\
\text { 5. Research KMC practice of mid to late pre-termers (>34 } \\
\text { week gestation) } \\
\text { 6. KMC barrier research } \\
\text { 7. Room L* 'snapshot' of practice (completed by me in } \\
\text { phase three) } \\
\text { 8. Models of practice: research nursing and organisational } \\
\text { hierarchy }\end{array}$ \\
\hline
\end{tabular}




\subsubsection{Discussion and Implications}

This phase of the research was extremely straight-forward to implement, with the majority of participants appearing eager and willing to discuss the programme within their unit. The use of semi-structured interviews as a method within a PAR framework was well aligned with PAR methodology and was intentionally framed by the prinicples of participation, empowerment and knowledge-sharing. As PARfacilitator, I consciously acknowledged each participant as an 'expert' of their own experience and 'held space' for democratising knowledge through giving voice to their perspective. One participant shared with real joy how good she felt to have the opportunity to talk about things that matter to her about the care of babies and whānau/families - including the fact that nobody actually had the time to focus on the 'important things'. I foreground the assumption that staff perspectives of KMC and the system which supports it, captured by interview, represent what is currently most important to the staff.

Analysis of the complex and often ambiguous data was assisted by the use of SWOT, a pragmatic and useful tool for categorising diverse perspectives without over-reduction of the data. An overarching theme of ambiguity emerged, which planted the seeds for a secondary discourse analysis for the identification of underlying power/knowledge networks. Reconciling ambiguity is likely to be a major aspect of all individuals associated with a NICU community. Vulnerability of the patients and their families, the diversity of practitioners within the workplace and some enduring historical aspects of the organisational model are all factors which contribute to complexity of this particular social system. Findings from this research showed competing values, priorities and frameworks acknowledged by the staff and common at all levels of operation, as well as both between and within individuals. Role ambiguity is one factor known to be associated with work stress, which in turn, is positively correlated to compassion fatigue and psychological burnout in nurses within the NICU setting (Barr, 2017). It is possible, though currently not empirically-based, that factors which contribute to perceived lack of social support, secondary traumatic stress and low compassion satisfaction in NICU nurses, result in chaotic and inconsistent KMC programme delivery (Barr, 2017; Chan et al., 2017; Seidman et al., 2015). It certainly appeared so in this unit. Furthering the inquiry into the theme of ambiguity found in this phase will be presented later in chapter six 
where there is more detailed analysis and discussion on the less visible aspects of KMC.

Sound rationale for identification of challenges and strengths of the KMC method for the purposes of quality improvement were provided for this SWOT analysis.

Findings from SWOT indicate that the staff are much more heavily focused on KMC themes influenced by organisational ( $\mathrm{n}=244$ responses) and 'human' factors $(\mathrm{n}=257$ responses), than they are on resources and the environment ( $\mathrm{n}=68$ responses). However, perhaps its usefulness in this case, in isolation from other phases of the research, was mostly found in the identification of potential 'next steps' for KMC quality improvement, represented in table six.

Based on findings from the interviews, when I began to invite engagement with the existing PAR group on the next phases of the study, it became clear that just two members of the initial group (both of whom had participated in preliminary meetings and interviews) were willing to continue to participate. One of the project ideas from the interview phase, research into KMC practice for predischarge infants, had the support of a second emerging PAR group (two from the original group and two from the interview phase). It was this that I moved forward with to facilitate the third phase of the exploratory cyle of the study.

\subsection{PHASE THREE: OBSERVATIONAL STUDY}
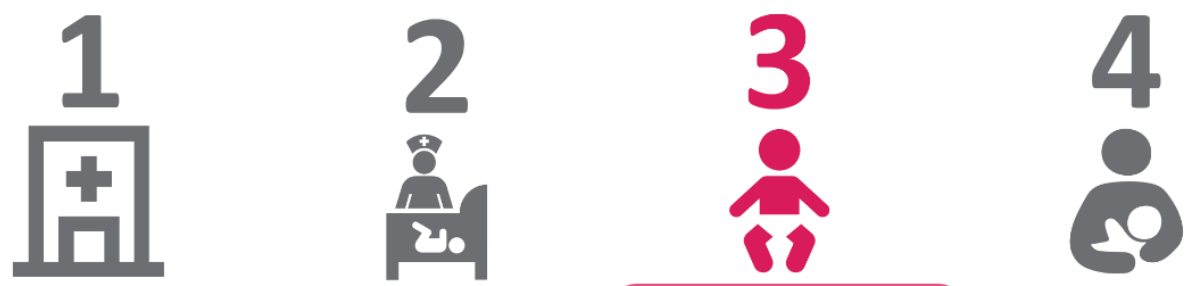


\subsubsection{Rationale and Research Questions}

Findings from staff interviews during phase two suggested that there was a gap in the use of KMC for babies once they were dressed, in cots and nearing discharge from hospital. Once babies are able to maintain their own body temperature, often around 34 weeks gestational age, they are dressed and moved from incubators into cots. Prior to this stage, babies in incubators wear just a nappy making skin-to-skin contact easier. Hence, the suggestion for further research into KMC for this group was made by one staff member, and supported by another three participants.

The rationale for the small observational study of KMC for predischarge infants was two-fold. Firstly, to provide a snapshot of KMC implementation through direct observation, thereby providing findings which were not available from the documentation audit in phase one of the project. Secondly, to provide observational data on the infant experience and some of the barriers and enablers to KMC for this group. Findings would add to the KMC picture for this cohort, providing NICU staff and management with valuable information for steering their own quality improvement process. A research proposal was initially submitted to the DHB governance group of concern, with the following research questions:

i. To what extent is Kangaroo Mother Care being implemented for babies in the pre-discharge phase of hospitalization within Room $\mathrm{L}^{1}$ ?

ii. What parental, staff, environmental and/or organisational factors influence the practice of $\mathrm{KMC}$ for this group?

\subsubsection{Participant Engagement and Research Ethics}

I sought feedback for my proposal to observe this cohort from all participants who had been part of the first two projects to this stage. By and large, the response was positive, indicating that the project would provide worthwhile information. Four participants agreed to actively engage with the design and/or facilitation of the project in various capacities. Two of the participants had been part of the first PAR group, now dispersing, and two had become engaged through the second phase interviews. The new PAR group of four, therefore, was made up of continuing participants as well as new ones. Once I was orientated on the unit and data

\footnotetext{
${ }^{1} \mathrm{NICU}$ room numbers have been assigned random identifiers to protect organisational anonymity
} 
collection had begun, all four participants became passive supporters for various reasons, yet remained available for consultation, if asked.

Three interesting sets of feedback to my proposal to observe $\mathrm{KMC}$ of predischarge infants were gleaned after inviting all participants from the study so-far to comment on the new proposed phase. Whilst these NICU members did not actively engage with the phase, I none-the-less considered them participants in a knowledge-sharing capacity. One participant, whilst voicing her theoretical support for the project, stated her reservations, citing the small size of the room, overcrowding, and lack of chairs as some reasons why KMC was not offered. In addition, the staff member, who possessed a $100 \%$ management role, felt that "nurses see getting a baby undressed as too difficult and time consuming, especially as they are trying to get babies to establish feeding etc" (R. Bear, field notes, p. 96, 5.11.2016).

The second participant who had been involved with phases one and two of the project believed that there were many priorities other than KMC for families with babies at this stage of hospitalisation. This participant was interested in the parental perspective about whether they would like to implement more KMC, and if so, how they could see it being promoted (R Bear, field notes, p. 97, 8.11.2016):

By the time that parents are in Room L they should be leading the care more and therefore I rely on them to initiate this [KMC]... Many parents will have their baby up for cuddles against their chests but with clothes on... And for babies and parents they are both happy... so at this stage of their discharge prep maybe this style of cuddle is as good as skin to skin?

Thirdly, one participant who had expressed strong advocacy for KMC in their interview felt the project was worthwhile and was interested in understanding more of the parents' perspective, for example, their home pressures, space, privacy and comfort of chairs in the pre-discharge room. Their questions included: "Is one of the barriers historical in that they were only allowed to do KMC once a day, and now we are trying to push rest between feeds as they near home? Can we facilitate KMC at any time that the parents want"? (R Bear, field notes, p. 97a, 17.11.2016).

My ethical obligations and intentions for this project were to ensure that minimal risk for harm was imposed on all participants of the study, including the babies, families and staff involved. Additionally, it was important that, on balance, the 
findings of the study would outweigh any risk to the most vulnerable of the participants, the babies and families. In effect, it was KMC (the intervention) that I was observing, rather than the subjects, and this was how the study was framed with respect to the DHB. Initial discussions regarding ethical considerations were had with the healthcare organisations' research office advisors, NICU operations managers; and the convenor of Victoria University's human ethics committee.

The range of possibilities for required ethical applications in this case was wide. I personally viewed the small study as low risk for causing harm, with a favourable risk:benefit ratio and aimed to secure approval for the study under the framework of the ongoing. Specifically, I proposed that the third phase study could 'roll on' under the umbrella of the original Victoria University's Human Ethics Committee approval (appendix 4), with organisational approval being sought through their existing quality improvement programme. Whilst there was some support for my application under these conditions, ultimately I was required to undergo full DHB review by the appropriate departmental governance research group (including re-application for special staff status, locality and external access agreements) (appendix 15); an amendment application to Victoria University's ethical committee (appendix 9); as well as a full scoping review through the Health and Disability Ethics Committees (appendix 1). The entire process took four months.

\subsubsection{Data Collection}

With ethical approval for observation of KMC for predischarge babies attained, a staff information sheet was emailed to the entire email list and I was asked to make contact with the Associate Charge Nurse Manager prior to each observation session. I constructed a 'Kangaroo Care observation chart' as a tool to collect characteristics of the room, handling, feeding and KMC of each baby in the room. In addition, I provided space to include comments about KMC or missed opportunities (appendix 14). The data collection tool was refined prior to the commencement of the study by testing it for two hours within one of the pre-discharge rooms. No individual demographic or identifying details were collected during the non-participant observation, therefore personal consent was not deemed necessary from either staff or patients' families. I did, however, display a 'research in progress' sign during observation sessions, with my details and those of my supervisors and University Ethics Committee for those who would like to know more about the project. 
Kangaroo Mother Care, handling and feeding characteristics, as well as environmental aspects of the predischarge rooms were collected over eight four-hour sessions, totalling 138 hours of individual observation involving 35 babies. Sessions were conducted on three weekday mornings, three weekday afternoons and two Saturday afternoon shifts.

There was a single incident at the beginning of the observation period where I participated in the care of a twin whose mother accepted my offer of help when she appeared to be overwhelmed by the requirements of her two babies. My role as nonparticipant observer primarily meant that I was to observe KMC practice without participation in the field of practice (Rook, 2017). Upon reflection of that session, I realised that the act of picking up a baby was not congruent with my non-participant observation method. Part of that reflection was to acknowledge the tension between my commitment to working in collaboration with the NICU community according to PAR and personal principles, whilst remaining a non-participatory observer as a parent and her babies were in need of and requesting my help. This paradox created moral distress for me. I also took into account the obvious unsaid displeasure of the caregiving nurse toward my actions, as well as an email from the team leader feeding back to me her own and two other staff members perspectives, asking me to reconsider my boundaries around cuddling babies, given that "it is not our policy for visitors to cuddle infants" (Email communication, R. Bear, field notes, p. 120, 12.5.17). This small example provides an insight into the moral and ethical dilemmas of working with multi-level and competing priorities within the research space.

\subsubsection{Analysis and Findings}

Data were analysed using descriptive statistics with a focus on KMC, handling and feeding observations and room characteristics. A summary of these statistics is provided in the following table: 
Table 8: Observation Statistics for Predischarge Infants

\begin{tabular}{|c|c|c|c|c|}
\hline & RANGE & AVERAGE & $\begin{array}{l}\text { PROPORTION/ } \\
\%\end{array}$ \\
\hline \multicolumn{5}{|c|}{ ROOM CHARACTERISTICS } \\
\hline \multicolumn{2}{|c|}{ Babies in room (number, \#) } & 2 to 6 & 4.5 & \\
\hline \multicolumn{2}{|c|}{ Cots in room (\#) } & 2 to 6 & 3.5 & \\
\hline \multicolumn{2}{|c|}{ Incubators in room (\#) } & 0.5 to 3 & 1 & \\
\hline \multicolumn{2}{|c|}{ Comfortable chairs (\#) } & 2 to 6 & 4.5 & \\
\hline \multicolumn{2}{|c|}{ Adults in room (\#/hour) } & 1.5 to 7 & 3.2 & \\
\hline \multicolumn{2}{|c|}{ Caregivers bedside (\#/hour) } & 0.25 to 2 & 1 & \\
\hline \multicolumn{2}{|c|}{ Average sound decibels $(\mathrm{dB})$} & 54 to 58.5 & 55.5 & \\
\hline \multicolumn{2}{|c|}{ Maximum sound decibels (dB) } & 65 to 80.5 & 72.5 & \\
\hline \multicolumn{5}{|c|}{ HANDLING AND FEEDING } \\
\hline \multirow[t]{3}{*}{ Bed type } & Cot & & & $n=27 / 35 ; 77 \%$ \\
\hline & Incubator & & & $n=5 / 35 ; 14 \%$ \\
\hline & Open incubator & & & $n=3 / 35 ; 9 \%$ \\
\hline \multirow[t]{6}{*}{ Feeding } & Breastfed only & & & $n=4 / 35 ; 11 \%$ \\
\hline & Breastfed/tube & & & $n=13 / 35 ; 37 \%$ \\
\hline & Breastfed/bottle & & & $n=6 / 35 ; 17 \%$ \\
\hline & $\begin{array}{l}\text { Tube }+/ \text { - total } \\
\text { parenteral nutrition }\end{array}$ & & & $n=7 / 35 ; 20 \%$ \\
\hline & Bottle & & & $n=4 / 35 ; 11 \%$ \\
\hline & Not observed & & & $n=1 / 35 ; 3 \%$ \\
\hline \multirow[t]{9}{*}{ Handling } & Cuddle - KMC & & & $n=1 / 35 ; 3 \%$ \\
\hline & Cuddle - conventional & & & $n=21 / 35 ; 60 \%$ \\
\hline & Cuddle - not seen & & & $n=13 / 35 ; 37 \%$ \\
\hline & Other - bath & & & $n=5 / 35 ; 14 \%$ \\
\hline & Other - weighed & & & $n=2 / 35 ; 6 \%$ \\
\hline & Other - cares* & & & $n=9 / 35 ; 26 \%$ \\
\hline & Other - pacifier & & & $n=6 / 35 ; 17 \%$ \\
\hline & Other - heel stick & & & $n=2 / 35 ; 6 \%$ \\
\hline & Privacy screen used & & & $n=9 / 35 ; 26 \%$ \\
\hline
\end{tabular}

*'Cares' refers to the group of routine activities performed by nurses and parents before feeding, often every four hours. For example, changing baby's nappy and taking their temperature. 
The primary research question for this phase asked 'to what extent is Kangaroo Mother Care being implemented for babies in the pre-discharge phase of hospitalisation within Room L'? The major finding was that negligible implementation of KMC occured during the period of 138 hours of individual observation. One out of 35 babies (3\%) was seen to experience $\mathrm{KMC}$ on one occasion, a baby in the first few hours after birth whose mother was still in a hospital gown and wheelchair, post caesarian-section. This baby was residing in room V, a room sometimes used for predischarge infants, and one of the few that I observed in an incubator (14\%). This may indicate that she was outside of the pre-discharge cohort of interest. The majority of the pre-discharge babies were in cots (77\%) and breastfed, with or without a bottle and/or tube top-up (66\%). The most common form of positive touch for this group was a 'conventional cuddle' (in the crook of a caregivers arm, or over their shoulder), clothed and/or swaddled, post-feeding (60\%). There was a significant proportion of babies (37\%) who were not seen to experience being held over the course of the multiple four-hour observation periods.

Environmental resources such as comfortable chairs, space, and screens for privacy were not observed to be limiting factors for the implementation of KMC. Neither were human resources (staff or parental) in short supply, or a factor in limiting the opportunity for babies to receive KMC. The babies were attended frequently by a caregiver during the observation periods, on average, once per hour. The next most common activity for the baby was remaining in their cot and receiving 'cares', that is, a nappy change, temperature check and observations (26\%). One medical procedure was observed - a heel stick which took place twice, both times while the baby was in their cot. Six babies were given pacifiers as a primary means of comfort, without either feeding or handling. Notable environmental characteristics included that the average sound level on this unit of $55.5 \mathrm{~dB}$, with an average maximum of $72.5, \mathrm{~dB}$ was too high by international NICU standard guidelines, as recommended by the American Academy of Pediatrics (AAP). The AAP recommendation for noise levels is below $45 \mathrm{~dB}$ during the day and less than $35 \mathrm{~dB}$ at night (Coughlin, 2014). In support of privacy, screens were available at all times and used by approximately one quarter of caregivers (26\%). Of the nine times screens were used for privacy, they were usually associated with breastfeeding and able to be sourced from within the room, when requested. 


\subsubsection{Discussion and Implications}

The observational study method itself was extremely straightforward and I would consider it to be a low-cost and simple method for adding valuable findings to a PAR study of KMC. By far the most complex and time-consuming aspect of this phase of the study was the ethical application process. My considered opinion is that the heavy time resource occupied by ethical applications for this study was a major determinant of the eventual requirement for its modification. Time to complete three iterative cycles of PAR within the scope of a full-time $\mathrm{PhD}$ project, simply ran out.

With regards to observational studies and the potential for uncontrolled observer (researcher)-related effect on the phenomena being observed, it is worthwhile to mention the 'Hawthorne Effect' here. Whilst critiquing this study, it may be said that as a KMC researcher, I may have affected the behaviour of the staff or parents and influenced the outcome of the inquiry in some way. Although a worthwhile perspective to consider, the basis of the Hawthorne effect is increasingly challenged by scholars, "given its multiple, contradictory, and imprecise meanings" (Chiesa \& Hobbs, 2008, p. 73). Indeed, through a PAR lens, it could be argued that as a tool for raising consciousness and self-criticality about one's own process and practice, the Hawthorne Effect may work advantageously toward quality improvement of KMC. There were, however, instances in my field work where the Hawthorne Effect may have intensified negative behaviours of nurses and nurse managers, including where my presence intensified conflict between a parent and caregiver. From an ethical perspective, therefore, it is worth considering whether this effect will add stress to any of the participants' experiences: "my impression is that my presence has had a negative effect on the behaviour of Nurse X... there is obvious tension between parent and nurse and the power dynamics are clear" (R. Bear, field notes, p. 117, 11.5.2017). For the purposes of the entire study, the effect is likely to be small enough to be insignificant, given that the data collection methods for the exploratory phases were largely non-observatory in nature (audit and interview), with the exception of phase three. Even with the observational study, the individuals in the rooms I studied appeared to adapt quickly to my presence, indeed, to often not even notice I was there. Whilst I can't say that the Hawthorne effect was zero, I would contend that it was negligible to the overall study findings, given the predominating absence of KMC. 
The use of this method would be suitable for a pre-/post-intervention type of observational study for a second implementation cycle of PAR. The application of a KMC educational intervention could be used, for example, with the parents, and then further KMC observations completed with the same group. Intermittent KMC observation could form a simple and low-cost part of an ongoing quality improvement programme.

\subsection{SUMMARY}

Phase one of the study began when the participants of a preliminary planning meeting asked me to facilitate a clinical case note audit comparing KMC policy guideline with documented practice. Due to insufficient detail in the case notes and observational charts, the primary aims of the audit could not be fulfilled. The most significant finding was that KMC was not documented for almost $60 \%$ of the days that babies were eligible to receive it. Even with the possibility that there was some under-reporting (the majority of participants believed this to be the case), this enormous number of missed opportunitites for $\mathrm{KMC}$ was alarming and requires further investigation.

Phase two findings from the SWOT analysis of NICU-staff interviews summarised strengths, weaknesses, opportunities and threats of their current KMC programme relating to aspects of the organisation, workplace culture, care paradigm, individuals and resources. The two predominant findings were firstly, overarching ambiguity across staff perspectives of their KMC programme, and secondly, staff suggestions for KMC quality improvement projects, one of which I designed and facilitated as the third phase of the study.

Findings from the third observational phase of KMC with predischarge infants confirmed the marked absence of KMC practice for this group of babies within this unit. Human and environmental resources such as attendance by caregivers, and available chairs and screens did not appear to be limiting. Noise pollution was established as an issue in all three of the rooms I observed, in accordance with anecdotal findings from the phase two interviews (multiple staff members were concerned about environmental noise). Chapter 5 continues with methods and findings from the two final phases, one parent and one staff-participant interview. In addition, the findings from a secondary review of local and global literature are 
CHAPTER FOUR: PAR, PHASES 1-3

presented in an effort to strengthen maternal and parental voice of their lived experience, to which I add my own. 


\section{SECTION B}

\section{CHAPTER FIVE: NICU NARRATIVES}

This chapter represents the perspectives from people who have a lived experience within the NICU, especially the voice of women and parents. Chapter 5 describes the fourth and fifth phases of the primary research, interwoven with 'other' voices from the NICU that were gleaned through secondary research findings. In the first case, I describe phase four of my research, where narrative inquiry was used to discover KMC-perspectives with the sole parent participant from this study. Next, in support of raising up the voices of women from this country (and in the absence of perspectives from other parent participants from my research) I describe the perspectives of whānau Māori (the indigenous peoples of Aotearoa New Zealand) through narrative findings in the literature. Then, to the other New Zealanders' perspectives, I add my own NICU-story. Following on, the findings from NICUstories of women and parents from around the globe are described, based on a decision to support my primary research through evidence from other studies. Lastly, the methods and PAR processes of the final phase of my doctoral project are detailed - those of the narrative inquiry with the longest enduring member of the PAR research group. 


\subsection{PHASE FOUR: PARENT NARRATIVE}
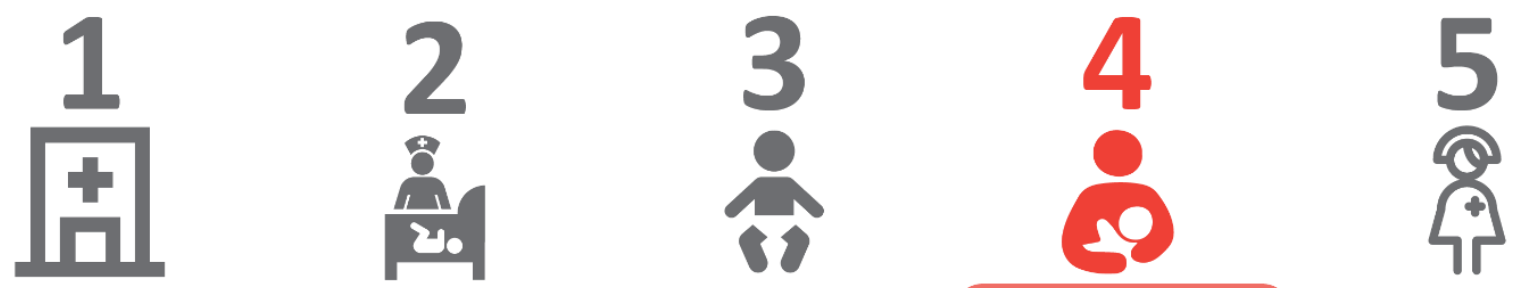

May-Jul 2017

Narrative

interview

Narrative

analysis

Narrative findings

\& NICU report

Discourse

analysis

\subsubsection{Rationale and Objectives}

Acknowledging parents as key stakeholders in the delivery of $\mathrm{KMC}$ and inviting them to participate in this study was essential to upholding PAR principles. This was continually foregrounded from the beginning of my interactions with the first PAR group and later the developmental care group. It was a shared perspective between myself and the PAR group that a significant 'piece of the puzzle' would have been left out of this study without consumer perspectives. To this end, during the time of my participation on the unit, I co-designed a survey of the parent's NICU experience in collaboration with developmental care group members. For mainly pragmatic reasons, the survey project was not completed. It wasn't until nearing the end of the third phase of data collection when another opportunity presented itself for consumer engagement.

Whilst observing babies' experience of KMC, one parent of twins expressed interest in sharing her 'NICU journey' and this was seen as one way to introduce parental voice through a narrative interview. Qualitative inquiry using narrative methods are increasingly being advocated for in health research (Blackburn, 2009; Marini et al., 2018). The construction of narratives are designed to "tell a story about some significant event in the informants' life... to reconstruct social events from the perspective of informants as direct as possible" (Bauer, 1996, p. 1). The rationale for 
the use of narrative inquiry from a constructivists' perspective is that ongoing cycles of writing, reflection, re-telling and re-writing deepen both the understanding and the meaning to both parties of what has been learned through experience - in this way the relationship creates a shared understanding of the meaning of someone's lived experience: "Being heard has created in him a sense of worth and a sense of personal power... the interview process has therapeutic benefits" (Koch \& Kralik, 2006, p. 10).

Objectives of narrative inquiry are to identify themes from the findings (Beck \& Woynar, 2017) as well as co-creating a narrative that stands alone as a complete finding (Adkins \& Doheny, 2017; Rossman et al., 2017). Shared story findings then have the potential to generate action-based change that is sustainable (Marini et al., 2018). The three-fold objectives for this phase were:

i. to address the phenomenon of a missing parental 'voice' for this KMC research;

ii. to capture consumer perspectives about their experience of KMC and the meaning they attribute to it; and

iii. to remain true to the methodological principles of PAR through the representation of all community 'voices', inviting shared power, participation and collaboration on a project which affects them.

\subsubsection{Participant Engagement and Research Ethics}

Ethical approval was gained through a fourth amendment to the original Victoria University's Human Ethics Committee application and it was agreed that it would be conducted after the parent's discharge from hospital. An information sheet and consent form were provided (appendices 16, 17). The interview took place two months after discharge.

\subsubsection{Data Collection}

The 60-minute in-depth interview was audio-recorded in the mother's home. An interview guide was prepared (appendix 18). In theory, the narrative interview is designed to minimise the input of the interviewer, resulting in a story-tellinglistening effect. It is acknowledged that any input from the interviewer will influence what the narrator will say next. This was precisely how the interview unfolded. The narration schema was completed by the question "what would be your blue-sky 
thinking (hopes and dreams) about KMC if this situation were to arise again?" The unfolding of the participant's KMC narrative surprisingly touched on all of the areas which I had questions about, requiring very little prompting, directing or leading by me.

\subsubsection{Analysis and Findings}

The fully transcribed data were analysed using a narrative approach, applying a theoretical framework that highlights personal, interpersonal, positional and ideological levels of understanding of a phenomenon - in this case, Kangaroo Mother Care. Two additional categories of analysis are found within this framework: firstly, interviewers are asked to be explicit about their responses to the perspectives of the narrator, thereby enhancing reflective and reflexive practices; and secondly, prominent discourses are identified and captured (Stephens \& Breheny, 2013). An example of the analytic process is shown in table 8 . Once analysis was completed, I constructed the participant's KMC story and sent it to her for verification. No amendments were made. The summarised narrative was then fed back to the NICU community within the NICU report. The participant chose her own pseudonym, ' $\mathrm{K}$ ' which has been used throughout the narrative analysis, findings and her final story. 
Table 9: Example of the Narrative Analysis Framework (phase 4)

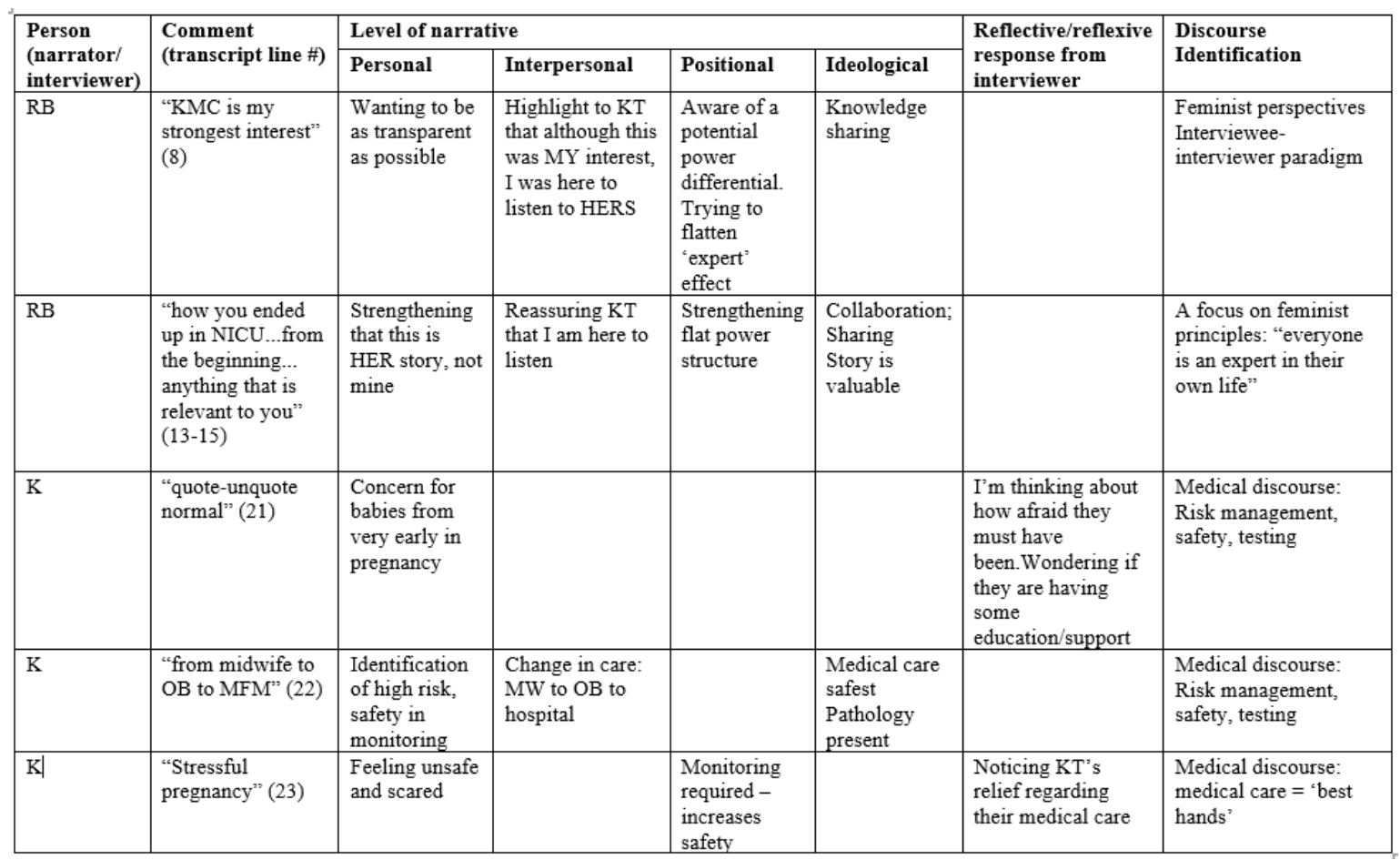

Predominant themes from K's narrative relating to KMC included:

i. the importance of KMC for bonding;

ii. the crucial role of $\mathrm{KMC}$ as positive experience for her babies;

iii. the importance of good communication for $\mathrm{KMC}$;

iv. more KMC information and education were desired;

v. more reassurance about her babies' physical safety if she slept during KMC;

vi. that she felt well supported to do KMC; and

vii. basic resources (comfortable chair, mirror) were usually available.

Summary of the narrative produced an overarching descriptor for K's KMC and NICU experience as "Being hospital-mother". To this, she attributed meaning about the distress she had faced, placing her difficulty with the hospitalisation of her babies, rather than becoming a mother to twins per se. Our co-narrative represents where improvements for her KMC experience may lie, with K expressing that extra support in two main areas would have been helpful. 
Firstly, in the area of education, she felt that more explanantion and information about Kangaroo Mother Care would have helped her to feel less, "in the dark about it... I would have been appreciative of that kind of information". Earlier in the interview $\mathrm{K}$ had noted that her experience of being taught how to care for her babies was "fairly informal... to the point of being a little unclear", whilst also acknowledging that she appreciated not being overwhelmed with information in the beginning.

$\mathrm{K}$ identified a second area of relating to her feelings of insecurity about physical safety during KMC, particularly while dozing, relaxing or sleeping. Simply being able to relax without feeling that she may "jerk and knock them off me". Internal conflict for $\mathrm{K}$ meant that she had concerns that it may be irresponsible and/or unsafe to sleep while in KMC with her girls, and she looked for reassurance to have felt more comfortable falling asleep... "not that I would have gone in there with that goal... but just to not fight it as much... it would have been more relaxing”. Here K identifies that in hindsight, to be more relaxed would possibly have helped her state of calm, resulting in her babies picking up on her "state of being". K suggested that encouraging comments would have been welcomed support during KMC, such as “your baby's not going anywhere... I can tuck a muslin around you... if you wanna close your eyes for 15 minutes... everything is safe".

A notable secondary finding was the therapeutic effect of the interview itself, noted by the participant in her feedback on the narrative method. $\mathrm{K}$ described her feelings about the process of 'NICU-storytelling' (R. Bear, field notes, p. 139a, 29.7.17):

Gosh, that was powerful to read... there is nothing in there that I feel is misrepresented or misunderstood. It rings true. I cried both times... I must admit that I had resistance to read it, probably a resistance to feel the sadness that I knew I would feel in reflection... However, it feels therapeutic to do so... So thank you for the opportunity to share! It felt like a healthy process. My closing comment is that it makes me realise that the thing I think that could most be improved about NICU is including mom's mental health as a vital part of the picture... it seems a glaring oversight or just a symptom of wider lack of of mental health support? NICU can leave you really... shredded.

At the conclusion of this phase of the study, and partly due to the narrative analysis process, my interest had become piqued about the discourses relating to KMC which could be used to examine underlying power relationships within the NICU community. It was at this time that I decided to apply a secondary analysis to significant amounts of the data collected in the first four phases and conduct a 
discourse analysis based on Foucauldian concepts. The discursive elements of the phase four narrative, along with those of the first three phases, were re-analysed and presented in chapter six.

\subsubsection{Discussion and Implications}

Narrative inquiry aligned well with the overarching PAR framework due to shared philosophical underpinnings. Scientific justification for the use of single stories combined with other qualitative and 'hard' (quantitative) data exists within the health research literature, enhancing the potential to capture psychosocial perspectives not available through other methods of inquiry. Given the participating parent's feedback on the narrative method used for this study, I believe that the knowledge she has shared has been both personally beneficial and highly informative to the group supporting KMC.

Whilst it is not possible to rule out the phenomenon of "strategic story telling" (Bauer, 1996, p. 10), whereby the narrators modify their story to tell the interviewer what they think they would like to hear, the frankness of the participant in describing both positive and negative perspectives in this case strengthen the findings. KMC was situated by this parent as a small, yet significant part of her whole NICU journey. Her memory of the KMC aspect of care appeared to have faded fairly quickly, and she expressed little conflict with her KMC experience, rather a more relaxed 'been there, done that' attitude. In addition, the 'rules' of narrative interview, when followed closely, help to guide the process in a way that mimimises interviewer impact on the story. By and large I felt that I achieved this by adopting a curious stance which centred on the person as expert of their own experience, avoiding providing opinion or judgements, not interrupting and not challenging the participant's perspectives.

In contrast, my own reflections from this interview reveal significant internal conflict due to my belief that this parent was not adequately informed about the 'whole truth' of KMC. In turn, she was providing less-than-optimal care for her infants without being aware of it and while thinking that she was adopting best practice. For example, K performed KMC every day or second day for just one hour, and stopped providing it altogether when her babies were dressed and in cots (around 34 weeks gestational age). When compared to the current evidence base on KMC, this parent 
was not fully informed and therefore not able to consent and choose in a way that empowered her to make decisions based on scientific understanding of infant development and best practice for infant care. As a result, I felt moral distress about wanting to advocate for her and her infants and others like them, based on my own knowledge, whilst honouring her own view that 'all was well'. This particular challenge, that of cognitive dissonance, was one that I was required to meet at many different points along the research journery. It was also one of the strongest motivators for inquiry into discourse and paradigmatic effects on KMC, which appeared to be largely invisible to the group.

Whilst there was much value in one parent narrative, it could be argued that one of the limitations of my research was a lack of participation in the PAR process by more than one mother/caregiver. One contributing factor to this limitation was pragmatic, due to the described barriers and difficulty of the PAR research process. I was aware, however, that there were likely to be additional societal, institutional and paradigmatic reasons for women's invisibility within the participating NICU of this study. In addition, there were just five published studies to describe maternal, parental and staff experiences of NICU hospitalisation within the context of Aotearoa, New Zealand, pointing to a gap in the knowledge base of parental and other service user perspectives. As the PAR process unfolded, a further objective emerged to expand understanding of the meanings women attributed to their NICU experience through representation of women's shared understandings from the local and global literature. In sections 5.2.1 to 5.2.3 I present narrative and qualitative findings from ‘other' NICU-parent graduates including Māori women and whānau, global voices and my own.

In summary, the first four phases of this project are able to stand alone as small studies in their own right, each requiring separate ethical approval from one or both of the institutions involved. Findings from each phase were used to inform subsequent phases, whilst keeping the original integrity of the PAR principles and the PAR-led methodology. The intention to capture broad KMC perspectives and observations from as many of the NICU community as possible remained my primary motivation. Finally, the results, conclusions and recommendations of the first four phases were described in a comprehensive report which was provided to all research participants for feedback. One participant, a key NICU stakeholder who had 
remained engaged with the entire project from the first point of contact, was willing to be interviewed to share her perspectives on the entire project, representing the fifth and final phase of the study (section B, chapter 5; 5.3).

\subsection{NICU NARRATIVES: 'OTHER' VOICES}

One of the objectives of my research was to 'hold space' for the perspectives of all NICU community members irrespective of their culture, role, or socioeconomic positioning within the NICU. Mothers, especially women identifying as indigenous to Aotearoa, were largely absent or found as problematic within staff discourse from this NICU, and minimally represented within the literature. It was important for me to represent maternal voice due to the feminist-PAR positioning of this research and the inherent over-representation of women in the NICU, an environment influenced by sociocultural, institutional and interpersonal power relations.

I searched three databases: CINAHL Complete; Proquest Nursing and Allied Health Source; and Victoria University of Wellington's Te Waharoa Library. Peer-reviewed articles published in English between 2000 and 2018 were identified using the search terms 'mother', 'neonatal intensive care unit' and 'narrative'. Where both parents' perspectives were given within a publication, these articles were included. A total of 273 papers were found on first search. Exclusions were made where: non-NICU environments were cited; observation of, rather than experiences by parents were described; duplicates occurred; and when there was a primary focus on fathering, neonatal death, or a topic other than the direct experience of the NICU. Forty-six relevant papers were included. Most papers were of qualitative design $(n=43 / 46)$. One unpublished public report produced by a kaupapa Māori researcher, Pihama (2010), was also included in the analysis due to its high relevance to the New Zealand context. Analysis and findings are summarised and represented under three separate headings as they relate to Māori voice, then global perspectives, and finally, to my own narrative.

\subsubsection{Whānau Māori voices from the NICU}

Indigenous women and whānau of Aotearoa New Zealand are more likely than nonMāori to have their babies hospitalised within the NICU, as well as have poorer medical outcomes for their infants (Ministry of Health [MOH], 2017; PMMRC, 2018). Inequalities continue to exist not only in the medical realm of care, but also 
relating to support by the predominantly non-Māori culture of the NICU, based on differences in worldview, spiritual and cultural values. This section summarises the findings of two kaupapa Māori researchers, Keri Thompson and Leonie Pihama. In Thompson's (2009) master's thesis, four Māori women and two whānau with experiences in the NICU told their stories. Pihama (2010) interviewed and reported the stories of thirty individuals and whānau. Findings from both narrative analyses identified common areas of distress and concern for whānau Māori during their stay on the NICU, including: bonding and separation; accommodation issues; isolation from baby and whānau; exclusion and restriction of whānau access to the unit; inconsistent experiences with NICU staff; lack of Māori staff; insufficient te reo Māori (language) and inability to practice tikanga Māori (customs); and external pressures such as distance to hospital, work and financial stress (Pihama, 2010; Thompson, 2009).

Participants in Thompson's (2009) research initially felt shocked and overwhelmed by the NICU environment. All women and whānau described how different NICUstressors, especially separation from their baby, affected the natural processes of bonding. All parents enjoyed Kangaroo Mother Care and felt that it enhanced their bond with their baby. Breastfeeding was difficult for four of the women, some of whom had to travel from out of town and were homesick and desired whānau support. From their stories, all women and whānau trusted and appreciated both the medical expertise and technological advances that supported their babies, whilst "wanting to support the medical care and interventions being made by the staff for the wellbeing of their babies" (Pihama, 2010, p. 6).

With regards to relationships, Thompson (2009) found that it was important to women and whānau that they had good relationships with staff. Women and whānau were very aware of the position of power that nurses held and desired to work collaboratively with them. Relationships were variably perceived. One woman and her whānau described being "treated like naughty school children" (Thompson, 2009, p. 91). In contrast, another family member described feeling "that his whānau were respected and able to retain parental control" (Thompson, 2009, p.93). One woman's experience through the NICU was viewed as negative and culturally unsafe due to "being questioned on the legitimacy of her whānau" (Thompson, 2009. p. 96). 
The importance of an advocate who understood the NICU and could enable clear communication was discussed by one woman, who in her case was her midwife. All women participants affirmed the vital role of whānau support for their NICU journeys, their stories revealing the importance of receiving the emotional support of family and friends whom they identified with. Other support people, such as NICUmothers and empathetic professionals, reduced stress by having an advocate and somebody to talk to.

Effective verbal and non-verbal communication were key to easing stress and proved supportive for parents who actively sought information about their babies. Narratives showed that clear communication helped whānau build confidence in their own caregiving capacities. Thompson (2009) concluded that "Parents need to know that they are heard" (pp. 91-92). Cultural miscommunication was a source of frustration and powerlessness for some women and whānau, with Thompson (2009) stating that a "critical component of efficacious communication with Māori requires the communicator to be culturally responsive" (p. 94). Pihama (2010) concluded her narrative analysis by emphasising that there were "clear issues raised in regard to social and cultural interactions within the units" (p. 6).

\subsubsection{Global voices from the NICU}

All 46 papers were read and analysed for predominating perspectives across the literature relating to women's experience of the NICU environment. The overarching parental and caregiving experience within the NICU was invariably represented by scholars as complex, stressful and requiring a huge adjustment from the expected start to parenthood (Ardal, Sulman \& Fuller-Thomson, 2011; Flacking, Ewald, Nyqvist \& Starrin, 2006; Aagard and Hall, 2008; Karatzias, Chouliara, Maxton, Freer \& Power, 2007). Findings were grouped into shared understandings within eight major domains: 'becoming NICU-mother'; 'claiming motherhood'; 'parental support needs'; 'NICU chaos'; 'emotional and behavioural states'; 'NICU relationships'; 'the importance of culture'; and 'spirituality'.

\section{Becoming NICU-mother}

Becoming a mother within the NICU environment was described as a distinct developmental process that progressed differently from 'usual' motherhood (Helin, 2015). Women attributed meanings to their experience by describing the need to adjust their expectations according to the loss of the ideal - a healthy full-term baby 
(Zani, Tonete \& Lima, 2014). Part of the adjustment included embracing the new normal of 'NICU-mother', including managing distress associated with interrupted bonding and separation from their babies (Higgins \& Dullow, 2003; Nystrom \& Axelsson, 2002; Pascoe, Bissessur \& Mayers, 2016; Tanimura, 2005). Twenty-eight Australian women were described as "struggling to mother" (Fenwick 2001, p. 52), at least partly a result of inhibitive nursing behaviours. Various other factors were found that affected women's perception of becoming NICU-mothers, including whether they were first-time mothers, part of the dominant culture, English-speaking and notably, whether they had experience as a NICU health-practitioner. Women described core beliefs about needing to protect, care and sacrifice for their infants, as well as beliefs about them and their babies needing to 'beat the odds' to reach the best outcomes.

\section{Claiming motherhood}

The process of developing and attaining a maternal role within the NICU was described by many scholars (Heerman, Wilson \& Wilhem, 2005; Helin, 2015; Higgins \& Dullow, 2003). Physical proximity, as well as being trusted and encouraged by staff to be the primary and active caregiver of their baby, was described by women as important to feeling like 'actual' mothers (Helin, 2015). Maternal role was often not attained until relatively late in hospitalisation or even until after discharge, especially where baby's health was compromised or women were distressed (Higgins \& Dullow, 2003; Wigert, Johansson, Berg \& Hellstrom, 2006). Communicating discontent about aspects of their babies' care was considered by women to risk being labelled by staff as difficult and was often avoided (Hurst, 2001 b, p. 44). However, some women seeking to assert their maternal identity chose to confront staff with their difficulties as their confidence grew.

\section{Parental support needs}

Women and parents talked about their needs for information, emotional safety, and 'being heard', describing the actions they undertook to get their needs met (Hurst, 2001a). In addition, parents described meaningful moments with their children and what constituted important first-time events for them (Wigert et al., 2006). The firsttime physical closeness of both skin-to-skin contact, and breastfeeding were important to one group of mothers for three reasons: they confirmed the strength of the baby ('a survivor'); the pair were closer to normality; the importance of the 
women as their infants' mother was confirmed (Flacking et al., 2006). Unfortunately for many parents, crucial experiences such as meeting their baby for the first time were accompanied by negative feelings (Wigert et al., 2006). Participants from multiple studies described how essential it was to reach out for support from various groups including family, friends, community and healthcare professionals. Peer support from others who had been in the same situation was reported as being important to women, particularly when their own families and friends were not very understanding (Rossman et al., 2017).

\section{NICU chaos}

The NICU was perceived as a "situational crisis" (Ardal, Sulman \& Fuller-Thomson, 2011, p. 93) by many of the women and parents involved with various studies. A combination of a lack of familiarity, the overwhelm of technology and fears about the health of their babies combined to constitute the NICU as a threatening 'foreign land' to many of the parents (Ardal et al., 2011; Broeder, 2003; Obeidat, Bond \& Callister, 2009; van Rooyen, Nomgqokwana, Kotze \& Carlson, 2006; Watson, 2010). Across studies, mothers' narratives represented how they felt physically and emotionally uncomfortable and out of place on the unit, including anxiety and concern with differences in caregiving that their babies received by healthcare professionals (Ichijima, Kirk \& Hornblow, 2011). The culture and environment of the NICU as a situational stressor may be even more heightened for women and families with non-dominant first languages and social determinants such as low socio-economic status and insufficient social support (Ardal et al., 2011; Pritchard \& Montgomery-Honger, 2014; Woodward et al., 2014).

\section{Emotional and behavioural states}

The spectrum, depth and complexity of parental feelings, emotional states and behavioural responses to NICU hospitalisation were broadly described as distressing, traumatic and often to the point of crisis (Adkins \& Doheny, 2017; Baum et al., 2012; Obeidat, Bond \& Callister, 2009; Pritchard \& Montgomery-Honger, 2014; Ribeiro et al., 2015; van Rooyen et al., 2006; Watson, 2011). Parents described "sadness, helplessness, disappointment, fear, frustration, and anger" (Obeidat et al., 2009, p. 27); "withdrawal, social isolation, and difficulty sharing their experience of the premature birth with others, as well as guilt, shame, and lowered self-esteem" (Baum et al., 2012, p. 596); and "a lingering, palpable sense of loss and resignation" (Adkins, 2017, p. 159). Contrasting emotions at the same time, for example both 
guilt and gratitude, were often described by women, with one researcher observing "vacillation between hope and hopelessness" (Obeidat et al., 2009, p. 24). In response to their difficult feelings, women and parents adopted a range of behavioural responses and states which included 'guarding' (withholding, withdrawing, self-blame), self-regulation (Fenwick, 2001), avoidance and social isolation (Baum et al., 2012), and submission to staff power and processes (Flacking et al., 2006).

\section{NICU relationships}

Across the literature, interpersonal relationships were described by women, families and staff as both potentially supportive and destructive. The profound nature of parent-nurse relationships was a topic that women attributed a lot of meaning to (Finlayson et al., 2014). Some neonatal nurses acknowledged the tendency for the profession to possessively safeguard the infants in their care (Watson, 2011).

Effective communication was considered by parents as vital to satisfying their need for information, knowledge and learning how to care for their baby (Miele et al., 2018). Some women felt that personality clashes were underlying ineffective communication, which could either make or break the days that they spent in hospital (Finlayson et al., 2014).

\section{The importance of culture}

Cultural context and responsiveness were described as strong influencers of parental experience, although specific details are currently not well researched in a diversity of cultures (Lee et al., 2005). A “cultural overlay" to mothers' experience in the NICU has been identified (Beck \& Woynar, 2017, p. 350). This may be especially so where there is intersectionality of various indicators involving race, ethnicity and wider sociocultural factors (Pihama, 2010; Thompson, 2009). Racism affecting the quality of care of infants and families was described in one US survey (Sigurdson et al., 2018). Based on Chinese-American immigrants' perspectives, researchers concluded that "minority populations with different cultural backgrounds and language barriers may have increased vulnerability in coping with their children's illness" (Lee \& Weiss, 2009, p. 274).

\section{Spirituality}

Studies conducted in Brazil, Israel, New Zealand and the United States described the importance of spirituality to women and families, with scholars noting that 
spirituality was evident irrespective of ethnicity, religiosity or education levels (Baum et al., 2012). Nine mothers from the United States revealed their dependence on prayer and "the maintenance of their spiritual self" (Schenk 2005, p. x). One researcher from New Zealand represented indigenous Māori women's NICU stories, describing the "spiritual connectedness that they [mothers] experienced by having whānau with them" (Thompson, 2009, p. 86).

\subsubsection{My NICU story}

My NICU-mother story is told with the passing of 16 years and the inevitable dulling of fine detail. My narrative has become shaped by the endless hours of contemplation in the arduous process of integrating and making meaning of my son Thomas' hospitalisation. Here I describe the personal, interpersonal, positional and ideological levels of my personal narrative that have created the overarching meaning and landscape of my NICU story.

On a personal level, I had emotional, intellectual and spiritual values and a nonnormative worldview that often intersected with NICU-culture. As well as the complex raft of distress, grief and roller-coaster emotions described by other NICUparents, I largely felt not 'unseen' within the NICU, conceding the way I desired to parent Thomas to the structures and processes of the NICU-system. To assert agency, express personal power and simply cope, I behaved with mild aggression, attempted to influence with my own knowledge, withdrew, and was often overwhelmed by despair. In my narrative, I wrote about my struggle to advocate for us:

I self-censored and learnt to pick my battles, including who to have them with... I conceded the way in which I desired to parent to the monolithic structure of the NICU-system... What I was always clear about was that $I$ was the most important advocate for my baby... it was me who was going to be taking him home and me who needed to form the healthiest and most enduring bond with him.

Interpersonally, relationships with many of my support people, including family and friends, were strained. I spent a lot of energy managing the ever-present potential for conflict and relationship breakdown. Reflecting on this, I wrote, "I believe that the majority of those relationships were unable to hold space for my vulnerability and maternal intensity as I became fixated on the most important relationship to me at the time - the one with my son". I coped by focusing on appreciating the efforts of the 
medical, nursing and family support I received, whilst being inwardly resolute about 'doing it my way'. In my narrative, I described it this way:

Not only was I out-of-town, but I was out-of-culture... Ultimately, despite lipservice to the contrary, I was clear that it was the nurses and doctors who held the majority of the power to care for and treat my baby and my opinion counted for very little. Not even my husband, family or friends seemed to understand the enormity of it all.

The ways in which I self-identified with various roles and positions in my life were also extremely influential to my NICU-experience, not permitting me to submit or comply absolutely with the routines of the hospital. There is meaning in my own narrative about:

how I identified myself as scientist, vet, second-time mother and holistic health advocate. My own positioning was swamped and drowned out by the larger discourse within the social context of the NICU. The narrative of the majority of nurses, staff and other parents reflected a wider social discourse that drew understanding from beliefs such as: 'Doctor knows best'; 'at least they're alive that's the main thing'; and 'thank goodness for the technology' (often symbolised by the incubator). The tasks, policies and protocols of the hospital reflected these beliefs and combined to produce mother-infant separation as the norm, something I was in abject disagreement with.

At the macro-level, broader social system ideology strengthened the biomedical system through a stance of 'NICU-as-saviour'. I attributed further meaning to this within my narrative:

The integration of psychosocial knowledge about mother-infant attachment, parentled care and culturally and spiritually responsive support were invisible and largely silent within secular society, translating to similar NICU-based trends of care. As a result, I experienced being marginalised and excluded from parenting my baby in a way that had most authenticity and integrity for me.

In summary, my overarching experience as NICU-mother was this. A sense of the NICU as an enormous and fixed system that made little room for women and mothers to express individual preference outside of the established biomedical norm. The paradigm which informed my own internal working model of mother, which required proximity to Thomas and close-to continuous KMC, required individual agency that was opposed within the unit. Whilst my feminist values held that individuals can and do enact their personal power in a multitude of ways, in this case, my cumulative knowledges fell steadfastly outside of the cultural NICU-norm. This created conflict and dissonance that were unresolvable for me at the time. The result for me personally was that the traumatic early birth of my son became even more laboured through his hospitalisation and subsequent opposition to my 
mothering style. I became aware of being positioned by staff in a negative way with inference that I was depressed, mentally distressed and a possible danger to my baby. My reality, however, was that once medically stabilised, it was the staff who were negatively affecting mine and my son's development by ensuring that we remained mostly separated by care practices that did not meet our biological needs. In response to this, I wrote:

It was this belief that distressed me most, and in that way, the greatest challenge to my wellbeing was iatrogenic, hospital-derived. As NICU-mother, too many moments of our 77-day stay were spent holding this unmanageable tension: that the life-saving and medically-necessary placement of Thomas in hospital was also the source of his increased suffering. 'First, do no harm' they say. Indeed.

\subsubsection{Discussion and recommendations}

The essence of the shared perspectives of these women, I believe, is the requirement for humanisation of NICU care in ways that attend more fully to the holistic needs of mothers, fathers and extended family groups. Where care is provided to infants based on their physical, functional and medical needs at the expense of mother's and families' psychosocial ones, suffering results. Redundant care philosophies and models have previously marginalised the necessary relationship-based emotional, spiritual and collective needs of the baby's mother and family unit. Now that women have been asked about their experience, it is essential that researchers and practitioners act to facilitate, through systemic change, what parents have called for. Integration of family-centred principles, in a timely and efficient manner, is required for the sake of improved outcomes and a more positive NICU experiences for the people whom it serves.

Many of the recommendations in the literature relating to parental experience of the NICU involved psychosocial, cultural and spiritual support. In addition, it was neonatal nurses who were believed to have the largest moderating role of parental stress and maternal role development. The current singular focus on the physical and medical wellbeing of the infant needs to be expanded by partnering with mothers (primary caregivers), by providing culturally-responsive developmental care for the mother, her whānau/family and her baby (Heerman et al., 2005; Obeidat et al., 2009; Thompson, 2009). The emotional needs of women to be physically close to their babies, have support to autonomously handle and provide care for them, and express agency as their baby's primary caregiver were strongly narrated (Wigert et al., 
2006). Meeting parents' cultural needs requires recognition that beliefs relating to prematurity and hospitalisation could differ widely between people of different cultures and intersect with the NICU culture (Lee et al., 2005). Spirituality was very important to some people (Baum et al., 2012). Explicit understanding of spiritual and cultural needs within each NICU context was recommended to provide a culturally competent environment, including the provision of linguistically-appropriate educational materials (Lee et al., 2005; Lee \& Weiss, 2009).

The parental need for near-continuous information provision was also described a lot, explained as an important part of confidence-building for parents when given freely by staff (Wigert et al, 2006). Effective communication was represented as ideal across the literature (Miele et al., 2018). Several studies described the positive effects of narrative writing as a mode of therapeutic self-expression as well as interpersonal communication (Kadivar, Seyedfatemi, Akbari, \& Haghani, 2017; Marini et al., 2018). In addition to nursing support, peer support through relationships with other parents with lived experience in the NICU was considered important (Ardal et al., 2011). To conclude, further research to expand knowledge of parental experiences of their NICU experience, especially relating to posttraumatic stress (Karatziaz, Chouliara, Maxton, Freer \& Power, 2007), emotional safety (Hurst, 2001a), culturally-specific concerns (Lee et al., 2005) and spirituality (Baum et al., 2012) was recommended.

\subsection{PHASE FIVE: PARTICIPANT-FACILITATOR NARRATIVE}
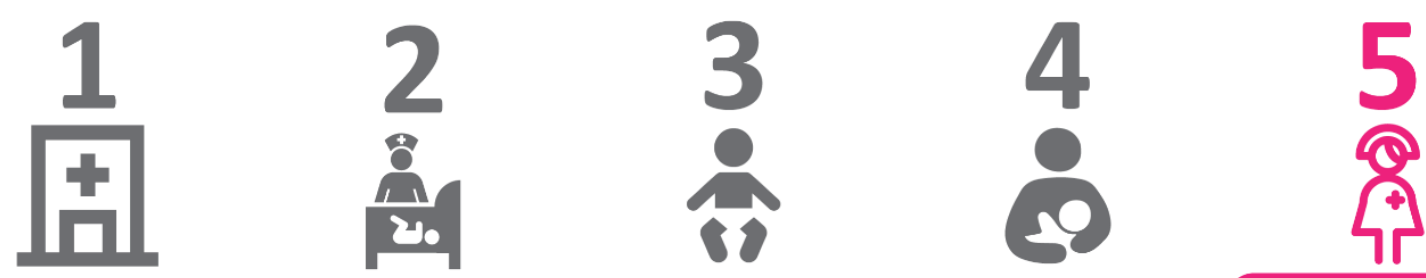


\subsubsection{Rationale and Objectives}

The final piece of fieldwork for this study was an interview with the one remaining PAR participant, a highly commited proponent of KMC in the NICU. The intention was to use the interview as a means of 'closing the loop' on the first exploratory PAR cycle of research. the objectives were:

i. to get feedback on the NICU report;

ii. to share perspectives on the KMC research findings, methodology and researcher 'performance';

iii. to glean ideas for future research cycles in this NICU (if any); and iv. to present my own ideas about predominant discourses influencing KMC within the NICU and receive participant perspectives on this topic.

I was interested in her perspective of both the KMC findings and the methodology used to inquire about it. Importantly, this narrative inquiry could provide an emic (internal) viewpoint, rather than one that is obtained solely through the perspective of the observer (Adkins \& Doheny, 2017). In addition, critical reflection of the project as a whole, within a supportive and collaborative space, offered further opportunity for increased individual responsibility and authorship of the findings according to PAR principles (Gorli, Nicolini, \& Scaratti, 2015). Perhaps paradoxically, the process of creating narrative is done in partnership and may be considered a co-creation, highly aligned with PAR principles. Ethical approval for the interview was covered by phase 2 of the study.

\subsubsection{Participant Engagement and Research Ethics}

The sampling frame for this phase included those individuals who had participated in the KMC project from its inception until its completion, whether directly or indirectly. There was just one person who fitted this criterion, and therefore a single discussion regarding an interview was had with the last remaining PAR participant. This person, a NICU staff member (pseudonym, Glenda), had remained engaged with the project across the entire timeframe of the research. I emailed her with a rationale and objectives for a final 'exit' interview, which was met with relative enthusiasm. I followed up with a copy of the proposed interview guide and questions (including my suggestion for her to feedback with any ideas for modification as they arose for her), and after one postponement, we met for our final interview (appendix 19). 
This phase of the study was the simplist and least time-consuming with regards to ethical approval. It was confirmed verbally and by email by both institutions that the applications made in phase two of the study would provide ongoing ethical approval for the final phase of the study, based on the nature of the interview, the low risk of harm to the participant and the individual consent for interview already given (appendices 4 and 13).

\subsubsection{Data Collection}

The interview was audiotaped and took place in the participant's office, lasting 90 minutes. After introducing the objectives of the interview, I maintained a narrative style of interview using active listening techniques and a non-judgemental, curious stance, guiding where needed for the purpose of fulfilling the interview objectives. Data were fully transcribed prior to analysis and a summary narrrative prepared. The narrative and full interview transcript were then sent back to the participant for feedback. One minor adjustment was requested, to 'soften' one comment that may have been misconstrued as inflammatory. The agreement between researcher and participant on the story construction was important due to its representation as a conarrative, thereby enhancing trusworthiness and credibility of my interpretation of our interview (Adkins \& Doheny, 2017; Rossman et al., 2017). The agreed conarrative stood on its own as a complete finding ('narrative as findings'), as well as a set of data for further discourse analysis (see chapter six).

\subsubsection{Analysis and Findings}

Data were fully transcribed and analysed using the same methods that were used for the parent interview in phase four. That is, a theoretical narrative framework was applied that highlighted personal, interpersonal, positional and ideological levels of KMC and developmental care, PAR methodology and discursive elements (table 10). 
Table 10: Example of the Narrative Analysis Framework (phase 5)

\begin{tabular}{|c|c|c|c|c|c|c|c|}
\hline \multirow{2}{*}{$\begin{array}{l}\text { Person } \\
\text { (narrator/ } \\
\text { interviewer) }\end{array}$} & \multirow{2}{*}{$\begin{array}{l}\text { Comment } \\
\text { (transcript } \\
\text { line \#) }\end{array}$} & \multicolumn{4}{|c|}{ Level of narrative } & \multirow{2}{*}{$\begin{array}{l}\text { Reflective/reflexive } \\
\text { response from } \\
\text { interviewer }\end{array}$} & \multirow{2}{*}{$\begin{array}{l}\text { Discourse } \\
\text { Identification }\end{array}$} \\
\hline & & Personal & Interpersonal & Positional & Ideological & & \\
\hline $\mathrm{RB}$ & $\begin{array}{l}\text { "nature of the } \\
\text { methodology... } \\
\text { critical } \\
\text { element" (11-) }\end{array}$ & $\begin{array}{l}\text { Difficulty } \\
\text { with critical } \\
\text { lens }\end{array}$ & $\begin{array}{l}\text { Inviting } \\
\text { feedback }\end{array}$ & $\begin{array}{l}\text { GL perspective } \\
\text { is important }\end{array}$ & $\begin{array}{l}\text { Seeking } \\
\text { feedback on } \\
\text { report } \\
\text { KMC-related } \\
\text { Methodology }\end{array}$ & $\begin{array}{l}\text { I am pointing to a } \\
\text { deeper meaning and } \\
\text { inviting GL to share } \\
\text { her understanding at } \\
\text { that level, including } \\
\text { language }\end{array}$ & $\begin{array}{l}\text { Collaborative } \\
\text { Partnership-based } \\
\text { paradox of } \\
\text { participatory } \\
\text { methodology being } \\
\text { critically-based }\end{array}$ \\
\hline GL & $\begin{array}{l}\text { "it doesn't work } \\
\text { so... push hard } \\
\text { to make it fit" } \\
(27)\end{array}$ & & & & & $\begin{array}{l}\text { GL aligning and } \\
\text { showing willingness to } \\
\text { engage }\end{array}$ & \\
\hline GL & $\begin{array}{l}\text { "culture, } \\
\text { feeling, } \\
\text { subliminal, } \\
\text { complexity..." } \\
\text { "... so much } \\
\text { more than } \\
\text { words, how do } \\
\text { you describe } \\
\text { that?" (34-) }\end{array}$ & $\begin{array}{l}\text { Shared } \\
\text { experience } \\
\text { with her own } \\
\text { research - } \\
\text { (frustration, } \\
\text { lack of } \\
\text { knowing) }\end{array}$ & Mutuality & & $\begin{array}{l}\text { Understanding } \\
\text { of ethnographic } \\
\text { and cultural } \\
\text { perspectives }\end{array}$ & $\begin{array}{l}\text { GL engaging with } \\
\text { sharing her own } \\
\text { experience }\end{array}$ & $\begin{array}{l}\text { Research } \\
\text { collaboration } \\
\text { Non-verbal } \\
\text { attributes of } \\
\text { communication }\end{array}$ \\
\hline RB & $\begin{array}{l}\text { "increasing the } \\
\text { trustworthiness" } \\
\text { "deeper } \\
\text { meaning" (47-) }\end{array}$ & $\begin{array}{l}\text { Impt to } \\
\text { articulate } \\
\text { principles of } \\
\text { research }\end{array}$ & $\begin{array}{l}\text { Communicating } \\
\text { imptce of } \\
\text { trustworthiness }\end{array}$ & $\begin{array}{l}\text { Stating research } \\
\text { position }\end{array}$ & $\begin{array}{l}\text { Connection to } \\
\text { principles }\end{array}$ & & $\begin{array}{l}\text { Imptce of } \\
\text { partnership in } \\
\text { research }\end{array}$ \\
\hline$R B$ & $\begin{array}{l}\text { "What does } \\
\text { absence of } \\
\text { KMC from } \\
\text { documentation } \\
\text { reveal?" (65) }\end{array}$ & $\begin{array}{l}\text { Stating own } \\
\text { finding }\end{array}$ & $\begin{array}{l}\text { Inviting } \\
\text { feedback }\end{array}$ & $\begin{array}{l}\text { Acknowledging } \\
\text { insight of 'the } \\
\text { other' }\end{array}$ & $\begin{array}{l}\text { Significance of } \\
\text { 'absence' }\end{array}$ & $\begin{array}{l}\text { Again here, inviting } \\
\text { feedback, partnership, } \\
\text { perspective of 'the } \\
\text { other' }\end{array}$ & $\begin{array}{l}\text { A counter-culture: } \\
\text { collaborative } \\
\text { research }\end{array}$ \\
\hline
\end{tabular}

The analytic process included deconstruction of the participants story into 'bites' of experience and meaning, represented in the text both implicitly and explicitly by: the presence of repeated patterns of thinking; emphasis on certain words or ideas; and perspectives about various individuals and their relationships (Adkins \& Doheny, 2017). The objective of the analysis was not to reduce the data to any sort of thematic representation, but to glean the participant's experiences of KMC, PAR research methodology and meaning attributed to KMC-related discourse. Once completed, the framework was used to construct a story which was sent to the interview participant for review and critique.

Findings from the narrative produced the overarching metaphor of: "A perfect storm". This was related to the trauma experienced by babies due to hospitalisation, maternal-infant separation, variable caregivers, and their impact on infant mental health. Other findings related to historical perspectives of neonatology that inform modern practice; underperforming KMC on her unit; and perspectives on discourse that impacted on KMC. These findings are represented in the following narrative, an excerpt from the full story:

As described in the introduction of this thesis, neonatology and the care of sick or preterm infants within a specialised unit of the hospital is a relatively new field of medicine and Glenda describes her understanding of the evolution of medical 
technologies that would enable the care of tiny humans, "to support someone to breathe, to eat, to prevent infection... to prevent skin damage". She does this by referencing some of the wars and significant global events which contributed to the production of such technologies. "In history, there were certain horizons that had to be in alignment before we could take another step [in neonatology]", she says.

Next, Glenda and I explored a small snapshot of developmental care of infants within her NICU setting. Specifically, how it historically (1990s) sat alongside medical care, yet "much to my distress in many ways... developmental care has almost been side-lined". Glenda believes that the focus on technological and biomedical care may have marginalised developmental care implementation in her unit, in addition to other factors, including, but not limited to:

i. increasing psychosocial complexities within New Zealand society and within the catchment area of this DHB. Glenda believes that "the social impact of New Zealand society today, on care in the neonatal unit, is huge";

ii. increasing mental health issues amongst family members;

iii. chronic understaffing;

iv. staffing by non-neonatal nurses;

v. a higher proportion of babies surviving at less than 25 weeks in the last decade;

vi. general increased acuity of babies and healthcare to medically manage them;

vii. risk-averse and task-based care behaviours by staff: "[social issues] at a subliminal level, impacting on nurses, who sometimes forget that they have, in inverted commas, 'normal parents' who really want to know about care" (participant transcript, Glenda);

viii. absentee parents; and

ix. insufficient funding from the Ministry of Health and DHB to operationalise a family-centred care framework: "there is no funding for the Mumma, because it is the baby that is the patient" (participant transcript, Glenda).

Glenda believes that a combination of these factors has led to reduced implementation of developmental care and KMC in the past decade. She is concerned that there are long-lasting consequences of not shifting the paradigm of care toward the family 'as patient'. She states: "I would love to see us not spend so 
much time and money in complex care and spend a bit more money and time on [infant] mental health and attachment”. She is committed to integrating developmentally supportive additions to medical care.

The perfect storm to which Glenda refers to in the NICU has been described by researchers as a constellation of three developmentally inappropriate factors that negatively impact on babies' neurophysiological development. Namely, parental separation, chronic environmental stressors and multiple painful procedures (D’Agata et al., 2017). Glenda describes her concern:

if we can't put a central person in there who only ever gives them positive touch and says, 'hang in there, we'll get through this together, you're safe, I love you'... if we can't do that... it's really scary.

Infant mental health and maternal-infant attachment theory underpin Glenda's motivation to increase awareness for developmental care, Kangaroo Mother Care, and the possibility of a 'Cuddle Aunt' programme. Glenda hoped that this research would add strength to the requirement for a louder 'voice' for infant mental health, the basis of which she believes is the primary relationship with the mother. Glenda was disappointed with the lack of Kangaroo Mother Care practice for the predischarge babies, saying "in some ways, I think huge amounts of damage can be done. But at the end of the day, that's all we've got". Here again, Glenda was aware that despite the best efforts of the NICU staff, the babies were enduring medical trauma and that a robust developmental care plan was needed: "when we can recognise trauma in war, we can recognise trauma in intensive care for an adult, why would we even think that a new little human being wouldn't experience trauma?"

Creating a 'shared language' was an important task, given that the voice of 'hard science' appeared to be a pre-requisite for getting your 'case read'. Glenda needed proof that Kangaroo Mother Care would reduce hospital stays. Glenda and I talked about the impact of referring to Kangaroo Mother Care as 'cuddles'. She suggested that the word 'kangaroo' may cause an aversion to the care method: "is kangaroo a word which makes people laugh - particularly in this country? We muse about whether the term 'cuddle' inappropriately relegates $\mathrm{KMC}$ to the realms of what Glenda describes as "nice to have, not a need to have" care? We agree that language is important and informing. Glenda suggests, that to raise critical consciousness and establish further interdisciplinary dialogue, perhaps it would be more appropriate to 
introduce KMC to parents and all staff as 'skin-to-skin essential intervention'. "Yea" she says 'I'd put 'essential' in. You know, it's how we fragrant up the language we use".

I then asked Glenda whether she believes that 'opinion-based', rather than evidencebased care is prevalent within the NICU. I articulate my own frustration about the lack of KMC for certain groups of babies despite its solid foundation in empirical science. In response, Glenda believes that there is a gap in neurodevelopmental knowledge in general. That it may not be on the agenda of the individuals that are directing much of the care. Part of the enduring barrier is that the evidence is still considered 'soft', and therefore less persuasive to the biomedical fraternity than 'hard' science. We had previously agreed on the continued silo-ing of knowledge, whereby softer interventions such as KMC are considered 'fluffy' and steered toward nursing. If that is the case, then it is possible that the biomedical enculturation of the unit results in the de-valuing of developmental care practice and insufficient KMC implementation.

This perspective then led us nicely into a discussion about the predominating discourses within the unit. A discourse analysis was included relatively late in the research based on my recurring observations of power relations on the unit. These were still 'inklings' at the time of our final interview and I was curious as to whether Glenda supported my initial views on discourse that were influential to KMC. I introduced proposed discourse categories at the interview for comment by Glenda and sought her feedback on the following: enduring techno-biomedical discourse; persistent hierarchical organisational style and predominating nurse-led care; baby as 'patient' and 'good-parent-bad-parents'.

Glenda believed that the historical influence of a hierarchical 'army-based' model of medicine had endured and that the neonatal environment has not yet 'outgrown' its influence. Within the context of her unit the "consultant experts at the top" direct things the way they wish to, with other inputs made by nursing specialists, based on a risk management model of healthcare. She supported the possibility that babies had been situated within the NICU as patients who requires nurse-led care, rather than as babies needing parenting, and that this enabled the ongoing task-based protocols of the unit. Positioning parents as a nuisance resulted in parents needing to 
seek permission to be included in their babies' care. In other phases of this research, parental marginalisation was identified as being caused by staff resistance to their presence at ward rounds and opposition to parental access to their babies' notes.

Glenda provided one example of predominating nurse-led care (rather than parentled care) as she described the routine weighing of babies at night by nurses. This routine provided information for the incoming doctors and nurses who need to make decisions about nutrient and fluid maintenance on the following day shift. Whilst this routine had become established in the 'workflow' of the unit, it was an important missed opportunity for mothers and families to experience skin-to-skin contact with their babies. About this, Glenda says:

I think part of it [resistance to change] is routine, because one of the routines we have is that the night staff usually do the weigh [of the baby]... it's something that they do two by two, you know, two nurses and a baby... in actual fact, it's one of the ideal times... surely, to strip the baby, give baby to mum to cuddle... [then] there is plenty of time to make the bed and do whatever.

As the mother of two hospitalised, preterm babies who came to know the 'NICU system' well, I expressed that I would have been uncomfortable with nurses taking the role of caring for my babies in ways that were nurturing. My perspective was acknowledged by Glenda, also offering that most parents would not question this routine: "how many mothers actually think, when they walk in in the morning and the weight is there, what has actually occurred to get the daily weight... how did you weigh this child?" Although there was the rhetoric of a parent-driven model on the unit, Glenda was sceptical about its application, "you know, you're [parents] part of our 'take care team'... you're the lowliest member of the care team". She would like to see more than 'lip-service' paid to partnership with parents - to explore what partnership means to the group. Multiple barriers to a parent-led model certainly existed and required addressing, not least the question of safety of babies who were born into families with complex social issues and/or poor mental health. Additional factors contributing to a lack of parental/caregiver engagement included family stressors such as travelling long distances, separation from other children and family members, and financial difficulty. Predominating societal 'mother-craft' discourse supporting maternal-infant separation and 'doctor as expert', may also feed into maintaining the status-quo of a nursery model of care within the NICU. It was these factors that Glenda believes have both frightened and created cynicism for 
professionals who question the possibility of anything but a pyramidal, expert-driven model of care. As well, Glenda believes that buy-in from parents for an increased level of input is likely to be low in their heterogeneous group. This was mainly due to the social stressors mentioned by Glenda, who estimated that up to $60 \%$ of families are 'wiped out' by these issues. The parental perspectives pertaining to these ideas remain unknown. Supporting nurses to continue their daily routines uncontested by not 'rocking the boat' may well suit a large proportion of parents and families who are already stressed and overwhelmed.

From a health advocacy perspective, one crucial question emerged. Are babies and families getting their biological needs met in the NICU at a critical developmental stage in their life course? Appropriate developmental care may be at the crux of differences between immediate survival, and long-term 'thrival'. It appeared to me, that parents and professionals would be unlikely to make clear and informed decisions if they didn't have all the information they needed. A gap in the education of Kangaroo Mother Care and developmental care for parents and multiple professional disciplines within the NICU seemed to be a factor in the current care practices within this context.

This research has been novel in this environment due to both the topic of interest and the methodology, and it was therefore heartening for me to hear from Glenda, our final remaining PAR participant, that the findings, to date, have: "been a big wake up, so if you're happy for me to use some of this to start coming back at some of our education and attitude and culture of the unit?" Feedback from Glenda regarding the research process, in general, reveals that topics for quality improvement are generally more practical and measurable in a quantitative capacity:

usually around compliance with policy which minimises risk... [for example] are we complying with hand hygiene audits, or are we complying with bundle-of-care for longline care? ... which is going to reduce our infection... something that has been identified and manageable... you're going to minimise iatrogenic [hospital-based] damage to babies.

My explicit difficulty with maintaining collaborative, relationship-based partnerships whilst researching with a critical lens was supported by Glenda who felt that openness to constructive criticism was unlikely to be encountered within her workplace. However, the value of honestly and respectfully sharing these findings, contentious or not, far outweighed any negative response received, and aligned with 
individual and group commitment to neonatal research. Indeed, Glenda's cultivation of her own critical and reflexive lenses and her willingness to share these perspectives with the rest of her team were prominent factors in the ultimate completion of this PAR research project.

\subsubsection{Discussion and Implications}

This co-narrative has provided insight into some societal, organisational, professional and parental/whānau/family factors influencing KMC within one NICU setting. The three-fold objectives of this phase were successfully met by the narrative inquiry in the following ways. Firstly, feedback was provided by Glenda on the summary NICU report regarding which direction she would like to take for future KMC research. Secondly, further KMC findings were gleaned, in addition to how she experienced the critical element of the methodology and the meaning she attributed to that. Thirdly, I was able to present my perspectives about prominent discourses on the unit with Glenda contributing her own experience and perspectives about discourse and power relations. The latter laid a foundation for further inquiry using discourse analysis, described in chapter six.

My experience with the use of narrative inquiry within the PAR framework was an extremely positive one. Upon reflection, I feel that the characteristics of narrative interview, when compared with the semi-structured interviews of phase two, allowed for a relatively deeper understanding between interview participants through the modes of story-telling, writing, listening and collaboration on the narrative. I suggest that narrative inquiry could be used as a tool in the first instance to build relationships with individual community members in the pre-planning phase of a project, when trust-building is crucial for the success of a project launch. My sense was that knowledge-sharing took place where there were common intentions and topics-of-interest and a willingness to reflect and raise one's own. From this fertile ground, the seeds of change may just take root and grow!

\subsection{SUMMARY}

The most observable aspects of this NICU's KMC programme has been described in chapters four and five through the findings from multiple phases of research that captured researcher and community member perspectives about KMC in their setting. In addition, further findings from women and parents who have lived 
experience within NICUs in New Zealand and around the world were included to strengthen the exposure of parental and whānau/family voice. For each of the five study phases, specific details have been described about each method of inquiry, including the rationale and objectives, participant engagement, ethics, data collection and analysis. The processes involved with data analysis necessarily followed the same attitude of PAR approach that was implemented throughout the entire study. Specfically, the emphasis was on participatory, action-orientated, problem-solving techniques which would derive knowledge of interest to the group (Kidd \& Kral, 2005), including methods which could be simply repeated for iterative cycles of the study.

Trustworthiness was principly enhanced through high reflexivity and collaboration with PAR participants, with as much participant input in each of the processes as possible. The use of multiple methods of data collection required multiple analytic techniques for its interpretation, the choices of which were based on pragmatic ideals of simplicity and ease of replication. Engaging participants with the analytic stage of the research process was unavailable for the entirety of the project, possibly due to the multiple and competing pressures on the group not lending itself to greater participant involvement. Given these pressures, simplicity and ease of replication were likely to be highly valued. Whilst absence of participation in the analytic processes of the study represented a departure from 'classic' PAR, the principles of participant collaboration were adhered to and allowed ongoing PAR methodology. Participant contribution and power-sharing was sought throughout each phase and largely made through PAR-group feedback on analytic findings, and brainstorming of the next phases of research design (Kidd \& Kral, 2005). Keeping the participant's needs at the centre, I propose that the use of simple analytic techniques provided for a repeatable process which could be used for further iterative cycles, if the group chose to do so.

In phase four, the KMC story of one parent's long NICU stay described both positives and challenges to her implementation of the practice with her twins. Whilst she felt largely supported to KMC for a window of time in the middle of their stay, there were incongruities with evidence-based knowledge which didn't sit well with me as a KMC researcher. The parent felt under-educated about KMC and would have appreciated more information about it. She also felt unsafe to completely relax 
and sleep while providing $\mathrm{KMC}$, which may have contributed to stressful 'unwanted thoughts' which sometimes made the practice unpleaseant for her. From the perspective given by this parent, it is my contention that while she appeared mostly satisfied about her KMC experience, she was not fully informed about the practice, resulting in its suboptimal use with her twins.

Next, the perspectives of women and whānau/family whose infants had been hospitalised in New Zealand and around the globe were represented. In addition, findings from my own NICU-narrative contributed to the evidence about what people experienced, what support was strengthening and what changes needed to be made. Women, their partners and wider families described their experiences of entering the NICU, claiming parenthood, useful support structures, and the importance of relationships, their culture and spirituality. Evidence suggested that people were asking for a more holistic approach to their care within the NICU in support of the necessary medical treatment of their babies.

Lastly, the interview with the longest enduring PAR participant produced a narrative with further findings on the KMC programme, influential discourse, and the use of PAR methodology for this study. Described as 'a perfect storm', Glenda believed that the trauma of hospitalisation often created conditions for poor infant mental health. Addtionally, she believed the developmental care paradigm which supported KMC has deteriorated over the last decade due to its marginalisation by biomedical and technological care. She would like to see the profile of KMC raised in the biomedical realm, where it had been positioned as 'fluffy' and non-essential. The participant agreed with my preliminary discursive findings that the hierarchical and 'top-down' system model within the unit works through power constructs of risk management and authoritative knowledge which gives lip service to parental partnership and has resulted in enduring nurse-led care. She also believes that marked social stressors have resulted in protective mechanisms such as task-based care which decreased parent-infant contact. Reflections on the PAR approach for this project found that the participant believed 'hard' (quantitative) science was more common and accepted, and there may be little openness to the constructive criticism that is inherent in the PAR methodology. 
Findings from the first four phases were related back to all PAR participants through a summary report. Feedback was encouraged and received by just one of the 17 participants and my offer of a face-to-face meeting, to discuss the results, was not taken up. I decided it was necessary to inquire more deeply into the meaning of the findings relating to paradigmatic aspects of their Kangaroo Mother Care programme. In combination with data collected from these first five phases, my own field notes and other KMC-related documentation, a further analysis was applied to data to explore prominent discourses within the NICU community relating to KMC. Discourse analysis brings us to chapter 6 and an exploration of power relations within the data, where its positioning within a PAR framework is described alongside the method and its findings. 


\section{SECTION C}

\section{CHAPTER SIX: DISCOURSE ANALYSIS}

By the end of two and a half years of field work within the NICU, I was interested to learn more about the ambiguous nature of some of the primary findings from the first five phases of the study. I was curious about the near-complete absence of KMC, in both the unit's documentation and from my observations. I was particularly interested in the inherent ambiguity and incongruence between what was being said and what was being done - a knowledge-practice gap - with regards to KMC. It was also evident that many in the NICU community and some of the PAR participants were not aware of this gap. This was evidenced by findings from the first five phases after which it became clear that many of the staff thought that KMC was being practised more often that it was. In addition to a knowledge-practice gap, there was an over-optimistic belief that they were implementing KMC to a far greater level than they were. The contradictory nature of the findings prompted me to consider a Foucauldian discourse analysis to explore how the practice of KMC was talked about in the NICU.

A combination of the call to go deeper with the lack of progress in supporting any meaningful KMC change opened for me the possibility to add another mode of inquiry. Discourse analysis summons researchers to examine power relations that underpin truth claims (Taylor in Wetherell et al., 2001). The French philosopher Michel Foucault (1926-1984), sought to examine how language and other modes of communication were used to establish meaning and systems of knowledge (and therefore power) within organisational daily practice. Academics interested in Foucauldian discourse analysis as a means of inquiring into nursing practice ask that "scholars work to discern what it is that goes unthought or unrecognized... what is known about a particular problem or concern, but is not expressed, what is ignored or covered over, and what is thought but left unspoken" (Springer \& Clinton, 2015, p. 88). The data I had gathered lent itself beautifully to the method of discourse analysis, enabling an inquiry into the power relations influencing the NICU 
community, particularly as it impacted on the KMC programme. The objective of the discourse analysis was to explore selected data in answer to the critical questions:

i. What are the discourses constituting the practice of Kangaroo Mother Care implementation within the context of this NICU?

ii. What is the wider sociocultural context that influence the practice of KMC in the NICU?

The beginning of this chapter describes my approach to discourse analysis which was informed by Foucauldian ideas around discourse-power-knowledge and overlaid through a critical feminist lens. I detail how this worked alongside PAR to enable deeper findings from the data which had been collected within the five individual phases of this study. Discourse analysis processes are described, most notably Foucault's' genealogical approach (Carabine in Wetherell et al., 2001), followed by a description of the findings. Lastly, a brief discussion of how the discourse analysis findings, the bones of the KMC programme, are integrated with the findings from the rest of the study to enhance the meaning of the available data.

\subsection{DISCOURSE ANALYSIS, FOUCAULT AND FEMINIST THEORY}

Discourse analysis presented as most suitable for this inquiry because there was marked ambiguity in the primary findings. This ambiguity pointed toward the presence of institutional power dynamics which were affecting the culture of the NICU in which KMC was taking place. A more in-depth understanding of the influential discursive elements which both knowingly and unknowingly influence Kangaroo Mother Care implementation within this NICU was therefore warranted.

When analysing discourse, Foucault (1972) asked a series of questions to decipher the laws operating within systems of relations, "discursive formations" (p. 38), asking:

i. who is speaking and who is qualified (has status) to do so?

ii. what institutional site do they speak from?

iii. how are subjects positioned in discourses and what spaces are possible for them to occupy? 
Foucault (1972), gave examples of answers to those questions relating to $19^{\text {th }}$ Century medical practice in this way (pp. 52-53):

The status of doctor involves criteria of competence and knowledge; institutions, systems, pedagogic norms; legal conditions that give the right - though not without laying down certain limitations - to practise and to extend one's knowledge... This status of the doctor is generally a rather special one in all forms of society and civilization... We must also describe the institutional sites from which the doctor makes his discourse... In our societies, these sites are: the hospital, a place of constant, coded, systematic observation, run by a differentiated and hierarchized medical staff... If, in clinical discourse, the doctor is in turn the sovereign, direct questioner, the observing eye, the touching finger, the organ that deciphers signs... it is because a whole group of relations is involved.

Weedon (1997) describes the importance of Foucault's theory relating to discursive fields which "consist of competing ways of giving meaning to the world and of organizing social institutions and processes" (p. 34). The matrix of the subjective field operates largely beneath the level of awareness of the individuals who operationalise them, presenting people with a range of subjective positions, not all of which "carry equal weight or power" (Weedon 1997, p. 34). Relating to the discursive field of the NICU, the figure who is imbued with the most status in the NICU is the paediatrician, speaking with authority from the dominant biomedical discourse (Golden, 2017). Biomedical hegemony is maintained through normative ideology of the dominant sociocultural group. Nursing and parenting discourses are also present, subjugated to the medical discourse through the more powerful authority of biomedical knowledge (Traynor, 2010; Wilson, 2012). Where minority discourses run counter to the predominating ones, they are likely to be "dismissed by the hegemonic system of meanings and practices as irrelevant or bad" (Weedon, 1997, p. 35). A history of biomedicine's present-day application of established discourse helps to provide a map of power strategies and understanding of where and how knowledge networks are embedded within the context of the NICU and is provided in subsection 6.3.1.

\subsection{DISCOURSE ANALYSIS AND FEMINIST PAR}

The scope and philosophy of PAR provide a framework for the adoption of any method of inquiry which aligned with PAR's epistemological stance and could respond to emerging findings from the study for ongoing cyclical inquiry. A brief search of the published literature found a small number of studies using discourse analysis within a PAR methodological framework. Scholars reported discourse 


\section{CHAPTER SIX: DISCOURSE ANALYSIS}

analysis as being synergistic with PAR frameworks “... often considered a methodology that aligns with participatory action research, feminist research... the intent behind the research is often related to changing social norms or power differentials" (Altman et al., 2014, p. 339). There was, however, an absence of literature describing discourse analysis for a PAR approach to inquiry in the field of biomedicine (Chappell, Rule, \& Nkala, 2014; Roy \& Ryba, 2012).

A feminist lens overlaid the discourse analysis, focusing on empowerment and the co-creation of knowledge. Taylor (in Wetherell et al., 2001) reminds us that the separation of research from researcher is impossible and assumes that the research process is affected at all stages by the researcher's stance and worldview. She goes on to say that reflexivity is highly valued, with the researcher's identity and role foregrounded and even utilised (p.17):

The researcher's special interests and, possibly, personal links to the topic are not in themselves a sufficient basis for research, but they are a probable starting point for the project. They are not seen negatively as bias but as a position to be acknowledged

Underpinning empowerment is the emancipatory premise that structures of discourse (and therefore power and knowledge) are multiple, intersecting and fluid. This results in possibilities for both oppression and privilege of all participants, depending on circumstances and subjectivities within the organisation at any given time (Griscti et al., 2017).

Like PAR, inquiry using discourse analysis sought to democratise power by increasing consciousness of discursive networks. Discourse analysis with applied Foucauldian lens and feminist perspectives also highly aligned with the PAR principles of consciousness-raising through critical inquiry for the purposes of individual and community empowerment and change (Kelly, 2005; Weedon, 1997). Leading on from these perspectives, a discourse analysis relating to $\mathrm{KMC}$ was conducted for the purpose of identifying power relations operating within the guidelines, practices and relationships of the NICU (Barrere, 2007). For these purposes, the NICU was assumed to be a complex and dynamic socio-political environment and "heterodiscursive" space (Springer \& Clinton, 2015, p. 91), implying complexity, the likelihood of ambiguity, and possibly even conflict. It was my assertion, then, that discourse analysis 'methodology as method' aligned well with the positioning of this PAR study. 


\subsection{DISCOURSE ANALYSIS IN THE NICU}

Embarking on a Foucauldian-informed discourse analysis began with gaining a thorough understanding about the genealogical method, followed by its application within the biomedical environment of the NICU. Genealogy applies an historical approach to analysis, noting the importance of historical aspects of the social context which inform the present-day situation. Described by one Foucauldian scholar, genealogy is "a history of the present... [concerned with] describing the procedures, practices, apparatuses and institutions involved in the production of discourses" (Carabine in Wetherell et al., 2001, pp. 275-276). The following section describes the historical context of how medical, maternity and paediatric discourses are relevant to the NICU and KMC practice. I then describe which documents I chose as data for the analysis, and why they were chosen.

\subsubsection{Genealogy of medicine, maternity and infant care}

Genealogy is a lens through which discourses are examined to reveal the power/knowledge networks which may endure to the present day. Genealogical inquiry into modern-day NICU functioning describes how networks of truth, knowledge and discourse are socially constructed, historically specific, and both produce and influence institutional power-relations relating to intensive care of babies who require it today (Carabine in Wetherell, et al., 2001).

In the latter half of the 20th century, post-world war two, the technological and professional advances of medicine was marked and "the cultural authority of scientific medicine grew" (Golden, 2017, p90). The life-saving effects of antibiotics, intravenous fluids, vaccines and ventilation were experienced, as was the enhanced credibility and authority of medicine as a profession of great social status (Golden, 2017; Wilson, 2012). The term 'technocracy' was coined by one prominent feminist scholar to describe the unfolding politicised model of modern medicine (DavisFloyd, 2001, p. S7):

\footnotetext{
a hierarchically organized society... [implying the use of] an ideology of technological progress as a source of political power... [expressing] not only the technological but also the hierarchical, bureaucratic and autocratic dimensions of this culturally dominant reality model.
}

The concept of 'professional' was simultaneously developed by regulatory bodies which affirmed medics' ethical commitment to their patients. They were authorised 


\section{CHAPTER SIX: DISCOURSE ANALYSIS}

with capacities of autonomous decision-making and considered to be in possession of a unique body of knowledge and expertise (Golden, 2017). One result of emerging medical orthodoxy was the establishment of physician-centrism and superiority, "medical professionals are viewed superiorly compared to professionals from other healthcare disciplines" (Wilson, 2012, p. 2), in conjunction with subservience of the nursing profession to medicine. Relating to the resulting hierarchy, nursing scholars have noted "an overall picture of severely limited (nursing) autonomy" (Traynor, 2010, p. 1506), including the historical gender bias of women in nursing and men in positions of power within the medical hierarchy (Kellett, Gregory, \& Evans, 2014; Rothfield, 2002).

Whilst a technocratic/patient-centred divide was a hallmark of this era, recent $21 \mathrm{st}$ century knowledge has called for "a conscious and critical integration of personcentred practice in the technologized care environments" (Lapum et al., 2012, p. 276). Healthcare scholars in the 21 st century acknowledge that "technological knowing is embedded as a dominant discourse early in one's (healthcare practitioner) career (Lapum et al., 2012, p. 281). There is, however, a recent and growing body of evidence which seeks to alter the course of overly reductive thinking in service of the whole human as a biological, psychosocial and spiritual being (Cheyney, 2008; Crowther \& Hall, 2015; Davis-Floyd, 2001; Garcia \& Yim, 2017; Morad, Parry-Smith, \& McSherry, 2013; Rothfield, 2002). A modern description of the biomedical space as being in a "chaotic state of affairs" (Wilson, 2012, p. 1) has resulted in an increasing call for a paradigm shift toward increased humanity and holism within global healthcare, for the purpose of enhanced quality of care (Marcum, 2008; Youngson, 2014; Youngson \& Blennerhassett, 2016).

Prior to this, early 20th century influences on maternity care involved the movement of birth from home to hospital and a change of maternity carer from midwife to doctor, a transformation that was marked and near-absolute (Lothian, 2001; Stark, Eremynse, \& Zwelling, 2016). Medicalisation of childbirth occurred in parallel with the phenomenon of 'scientification of motherhood', whereby mothering practices, including infant feeding and child-rearing, became extensively medicalised, a phenomenon Foucault (1973) reported as being under the "medical gaze" (Alianmoghaddam, Phibbs, \& Benn, 2017, p. e289). The "dominant discursive imaginary” (Reiger \& Morton, 2012, p. 175) within maternity care was the 


\section{CHAPTER SIX: DISCOURSE ANALYSIS}

biomedical one, enduring well into the 20th century. As a result, feminist critique has described the theme of "devaluing and depersonalizing of women's experiences of childbirth and infant feeding" (Ryan 2001, p. 486).

Importantly, a profit-based relationship in the Western world centred on the marketing of infant formula, further incentivising the position of the healthcare profession as monitor and expert of infant care (Alianmoghaddam et al., 2017). For both obstetric and neonatal care of even the 'normal and healthy' baby, advancing institutionalisation and professionalisation resulted in increasing separation of babies from their mothers with the use of nursery settings, a phenomenon which is now considered scientifically outdated (Abel, Park, Tipene-Leach, Finau, \& Lennan, 2001; Bergman, 2014; Davis, Mohay, \& Edwards, 2003; Golden, 2017; Memo, Longo, \& Soriani, 2011; Neczypor \& Holley, 2017; Phillips, 2013; Stark et al., 2016).

The specialised medical care of the medically fragile infant occurred solely within the NICU from the early 1960s, where it evolved first in the United States (White, 2011). Since this time, scholars have described the phenomenon of biomedical dominance in this setting, with the increasing marginalisation of the "subjective, feeling aspects of its patients" (Rothfield, 2002, p. 320). By the late 1980s, the discursive construct of 'preemie' was identified, a new "category of person" linked in the eye of the public with their "principal caretaker, neonatal medicine" (Landzelius, 2006, p. 670). The changing landscape of maternal and infant healthcare in contemporary times include descriptions of humanistic paradigms of care toward a more integrated developmental approach (Als et al., 2011; Altimier \& Phillips, 2016; Bergman, 2015; Davis et al., 2003; Hall et al., 2017; Maghaireh, Abdullah, Chan, Piaw, \& Kawafha, 2016; Nyqvist et al., 2010a). In addition, the situation of 'patient' as 'consumer', with accompanying power to participate in decision and policy-making has afforded the possibility of increased autonomy and informed consent (Reiger \& Morton, 2012).

Concurrently, government-based bureaucracy in the 1990s saw a move toward 'managerial' control of doctors within healthcare systems, particularly within state and insurance funded organisations in the United Kingdom and United States of America (Traynor, 2010). Next, challenges to medical professional autonomy 


\section{CHAPTER SIX: DISCOURSE ANALYSIS}

resulted from the trend toward evidence-based medicine and questions over 'opinion-based care', the 'art of medicine' and the liberty of medical doctors to follow non-scientifically based medical tradition and folklore (Golden, 2017). Foucault (1978) used the term "bio-power" (p.140) to describe the increased tendency of governments to exert power and control on individuals. Foucault (1978) linked bio-power with the emergence of capitalism in this way (pp. 140-141):

This bio-power was without question an indispensable element in the development of capitalism; the latter would not have been possible without the controlled insertion of bodies into the machinery of production and the adjustment of the phenomena of the populations to economic processes.

Discursive strategies then arose out of the mandate for healthcare institutions to reduce public health costs. This, in turn created further power relations between state, institution and individual (Alianmoghaddam et al., 2017).

In summary, inquiry into the genealogical factors relating to discourse and power/knowledge in the NICU reveals the historical prominence of a highly politicised biomedical system. Social status has been attributed to physicians within a hierarchy that privileged their expert knowledge as authoritative, to the exclusion of other practitioners. Highly relevant to this study is the effect of medical discourse, summed up as 'Doctor knows best', as birth and maternity care came to rest under the medical gaze within the hospital setting. The medical profession became situated by the predominating biomedical discourse as experts in infant care and mothering became increasingly influenced by biomedical authoritative knowledge. In addition, governments increasingly established powerful positions of authority through the strategies of managerialism and institutionalisation of health (the health 'industry'). The next step in examining the power relations affecting KMC within the NICU of this study was to decide on which data would be the subject of analysis.

\subsubsection{Documents as data}

This section makes explicit the assumptions I made when choosing which material were considered data for the discourse analysis (Taylor in Wetherell, et al., 2001). Although analysis of documentation for influential discursive elements is not new to the field of nursing, not all documentation text is of relevance to the analysis (Rook, 2017). Taylor (in Wetherell, 2001) heeded the warning "not to approach the analysis with the assumption that whatever material is to hand somehow contains revelations, 
like gold within the dross" (p. 24). I based my choice of data for this analysis on the following theoretical assumptions, adopting recommendations made by Taylor (in Wetherell et al., 2001).

i. Context and background information were considered relevant, yet separate to the documents considered for analysis;

ii. Language was viewed as reflective of the NICU members' views, skills and knowledge;

iii. Data produced both by and for the entire NICU community was selected as broadly and inclusively as possible;

iv. Only documents highly related to the topic of KMC were considered, which included texts of wider social practices which may influence KMC;

v. Both the presence and absence of KMC-associated terms were equally informing in the data used for this analysis.

Relevant documentation used for this analysis included research participant emails, organisational policies, procedures and guidelines, my own field notes, and raw data from the first five study phases including staff, parent and participant interview transcripts. As recommended by other discourse analysis scholars (Altman et al., 2014) it was important to include both existing organisational texts and data generated by this research, which is what I did.

A preliminary overview of the texts using Kangaroo Mother Care as the 'object' and NICU community members as the 'subjects' established discourse categories which were discussed with the last remaining PAR-participant. The purpose of this "member-checking" (Altman et al., 2014, p. 344) was two-fold. Firstly, to remain aligned with PAR methodology, inviting the perspectives of research participants with respect to the findings arrived at by the facilitating researcher. Secondly, to enhance the scope and meaning of the unveiled discursive findings through validation and/or rejection by the other participant, further establishing a basis for data exploration (Altman et al., 2014).

\subsection{THE DISCOURSE ANALYSIS PROCESS}

Foucauldian scholars echo that there is not a single 'best way' for performing a discourse analysis. Carabine (in Wetherell et al., 2001) states that "Foucault didn't 
provide us with a 'how to' guide to genealogy" (p. 269) and Douche (2007) notes that "there is no one method for analysing texts to identify discourses" (p. 115). Despite this, researchers in the field have described the necessary elements to produce a thorough and informative inquiry (Carabine, in Wetherell et al., 2001).

I examined the data for KMC-related discourse using an inductive approach informed by two lenses. Firstly, the analysis was framed using the questions asked by Foucault (1978) previously listed on page 180. Secondly, Davis-Floyd and St John's 'Tenets of three medical models' framework was used (table 11). The rationale for the use of the latter was that from the beginning of the study, I had been developing ideas about the presence of a paradigmatic clash between the dominant biomedical hegemony of the NICU and a more holistic model required for the facilitation of KMC. I have used the technocratic/humanistic/holistic framework proposed by Davis-Floyd and St John (2001), adapted to the specific context of the NICU. Minor changes to the framework consider the phenomenon of the biopsychosocial interconnectedness of the parent-baby dyad, labelled as parent/patient for the purposes of this study (Davis-Floyd et al., 2011). 
Table 11: Tenets of Three Medical Models, adapted for the NICU

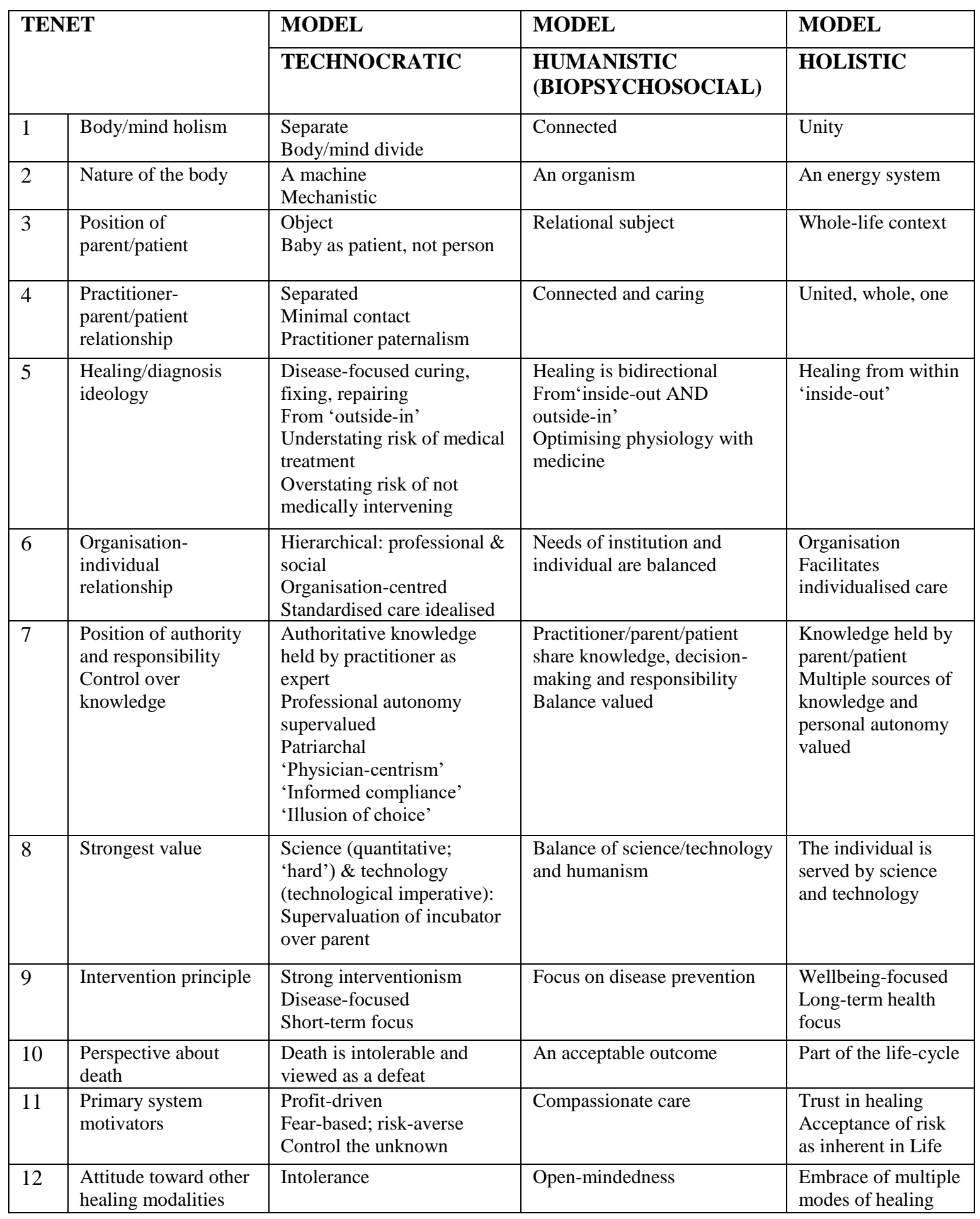

Adapted from Table 1 "The three paradigms: the technocratic, humanistic, and holistic models of medicine" by R. Davis-Floyd, 2001, International Journal of Gynecology \& Obstetrics, volume 75, p. S21. Copyright 2018 by John Wiley and Sons No. 4266990196688. Reprinted with permission. 


\section{CHAPTER SIX: DISCOURSE ANALYSIS}

Scholars of Foucault recommend examining data for the following characteristics: discourse categories and themes; discursive strategies and tools/techniques; situation of subjects; and the effects of the discourse of the subject/object/community (Carabine in Wetherell et al., 2001). Selecting an explicit topic of focus was the first and simplest step, namely the individual and community discourse(s) constituting choice to implement KMC. Broader developmental care themes which potentially impacted KMC were also included based on the interconnectedness of the two topics. The next step was to decide what the sources of data were going to be and to familiarise myself with them to aid in their analysis. One benefit of using some of the documents that I had worked with in the primary analyses was that I was already very familiar with the data. I chose to use the text present in the following: participant emails; KMC guideline policies (nursing/medical staff, parent/caregiver); parenting information sheets; a student nurse guide; my field note diary; staff and parent interview transcripts; and observational study field notes. In addition, I remained open to environmental information relating to KMC within the NICU, such as posters, photos, promotion and advertising.

Next, I began the process of identifying discursive categories and themes which included the inter-relationships between discourses and subjects, as well as the resulting effects of the discourse. The documents were examined line by line for language and context which related to the topic. I used an iterative process of reading and re-reading the data, remaining open to emerging categories, whilst being reflexive about the influence of St John and Davis-Floyd's medical models (2001). Two of the three medical models described by St John and Davis-Floyd emerged as categories from this analysis; both technocratic and humanistic discourse were present, with the notable absence of any holistic discourse. Guided by Foucault's (1978) premise that what is not said may be as informing as what is said, the absence of holistic medical discourse was equally as significant as the categories and themes found to be present. Foucault (1978) theorised about the importance of silence in this way (p. 27):

Silence itself - the things one declines to say, or is forbidden to name, the discretion that is required between different speakers - is less the absolute limit of discourse... than an element that functions alongside the things said... There is no binary division to be made between what one says and what one does not say; we must try to determine the different ways of not saying such things, how those who can and those who cannot speak of them are distributed, which type of discourse is authorized, or 


\section{CHAPTER SIX: DISCOURSE ANALYSIS}

which form of discretion is required in either case. There is not one but many silences, and they are an integral part of the strategies that underlie and permeate discourses.

The discursive strategies, techniques and tools were also identified and together these findings captured and summarised in a table to aid in communication of findings, albeit too extensive to include in the body of this thesis. Lastly, acknowledgement of the limitations of the data and research are foregrounded.

As facilitating researcher, I acknowledge my own personal subjectivity involved with the interpretation of the data, largely due to my own experience as 'NICUmother', my previous involvement with research in the NICU and my in-depth knowledge of infant developmental biology and KMC. In common with other interpretive methodologies, researcher subjectivity is not considered prohibitive for participatory, feminist and discourse analysis approaches (Altman et al., 2014; Jenkins, 2015). This is mainly due to the social science acknowledgement of subjectivity and multiple truths, whereby "the myth of objectivity is being dismantled" (Barbera, 2008, p. 141). Instead, characteristics of reflexivity, relationship-building and participation are lauded. That said, I would like to acknowledge that findings from this analysis are likely to be interpreted by some as contentious and that my intention remains to create constructive engagement for the purpose of quality improvement of KMC within this environment.

\subsection{DISCOURSE FINDINGS}

In the next sections, discourses relating to KMC practice are presented through a Foucauldian power/knowledge lens. In addition, the discursive effects and positioning of NICU community members are described. Direct quotes from the data and relevant documentation are used to strengthen the findings which were also discussed with the one remaining PAR-participant. Due to the large amount of data and findings, the results are summarised in table 12 below, followed by brief discussion.

I have organised the findings into five predominating discourses, each with multiple discursive themes, beginning with the most prominent:

i. biomedical technocracy;

ii. health system discourse;

iii. humanism; 
CHAPTER SIX: DISCOURSE ANALYSIS

iv. nursing authority; and

v. nurture discourse. 
CHAPTER SIX: DISCOURSE ANALYSIS

Table 12: Summary of Discourses and Themes

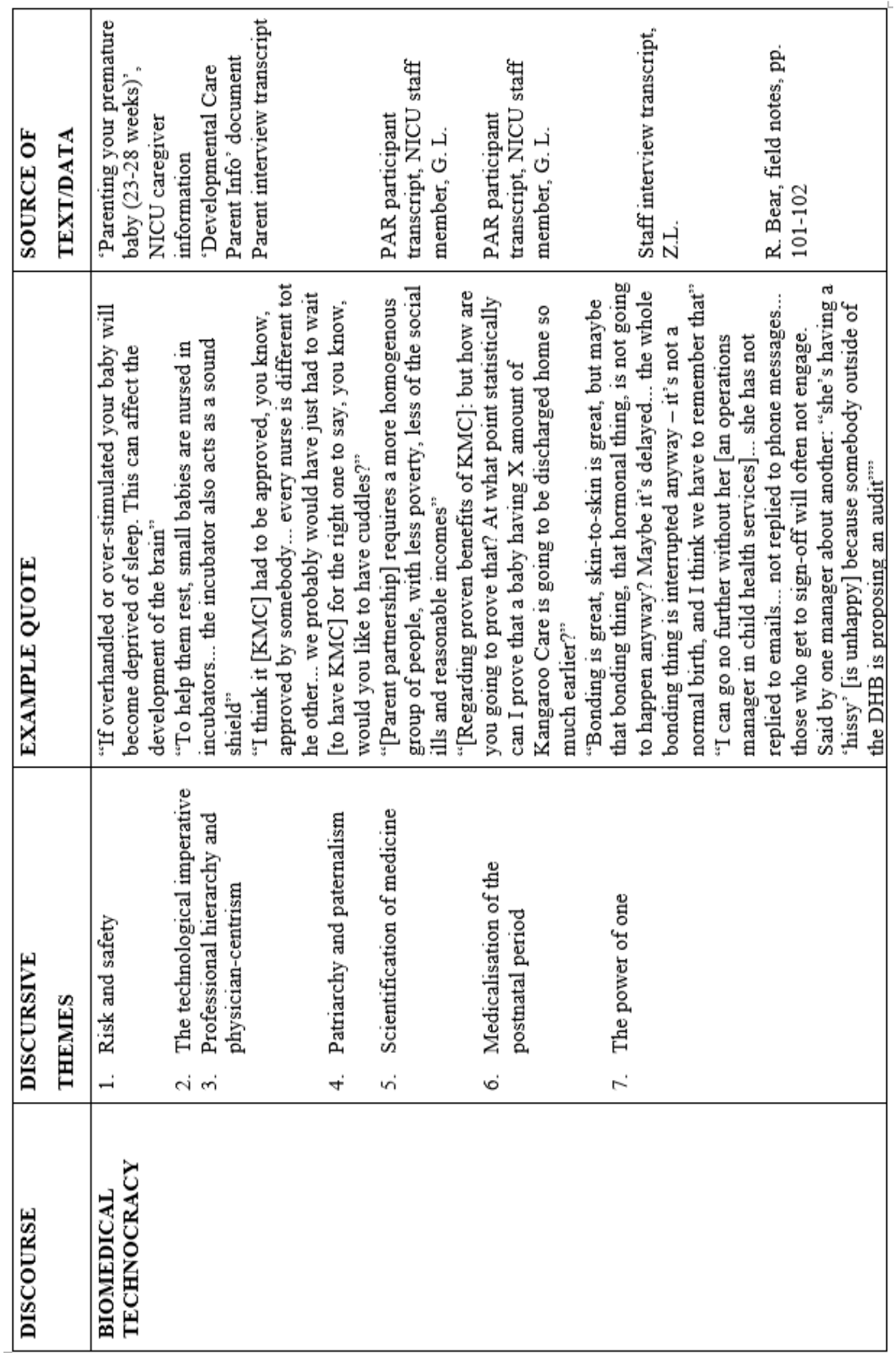




\begin{tabular}{|c|c|c|c|c|c|c|c|}
\hline 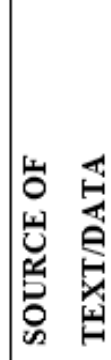 & 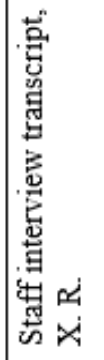 & 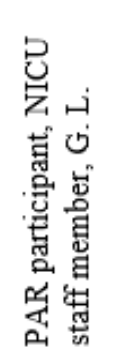 & 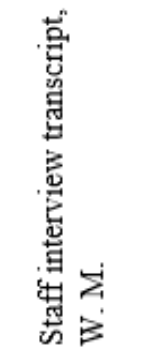 & 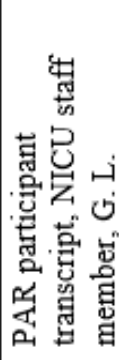 & 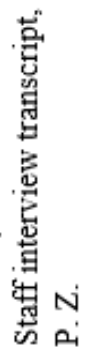 & 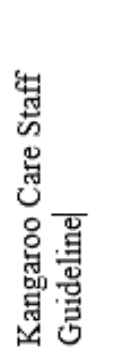 & 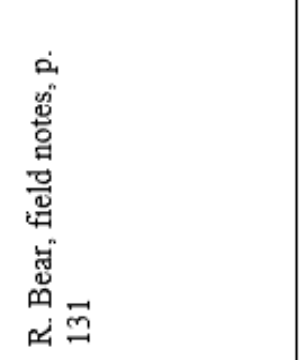 \\
\hline 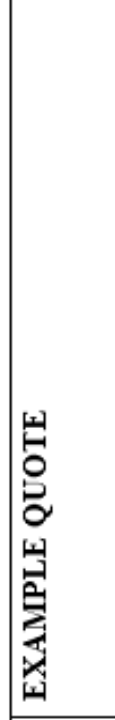 & 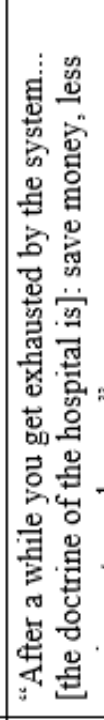 & 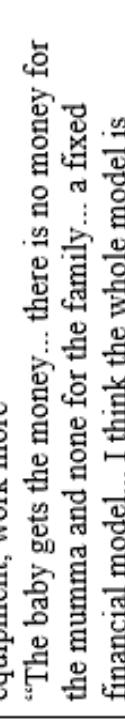 & 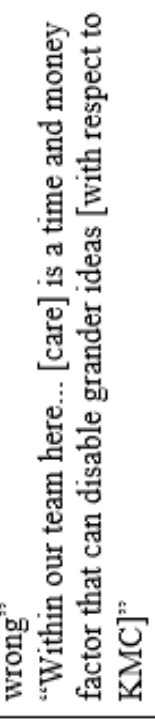 & 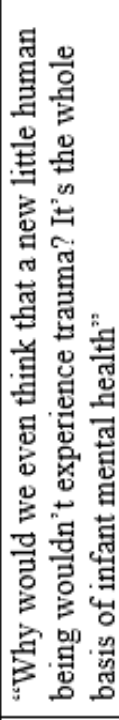 & 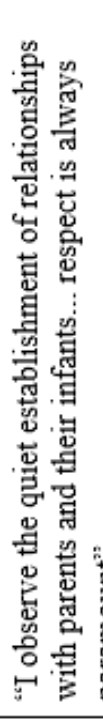 & 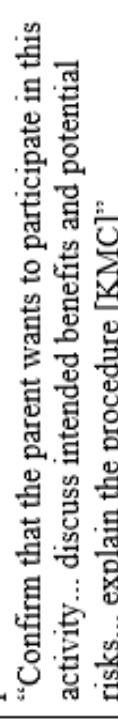 & 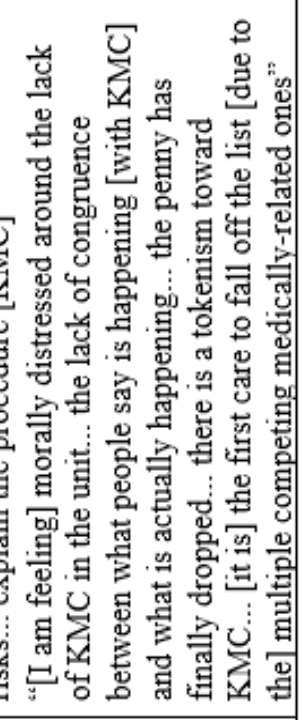 \\
\hline 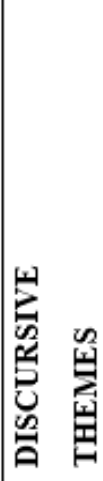 & 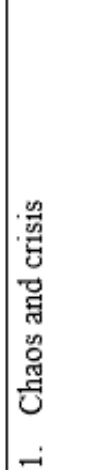 & 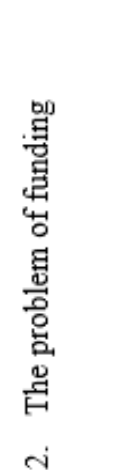 & 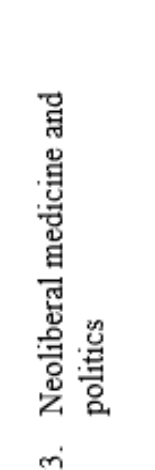 & 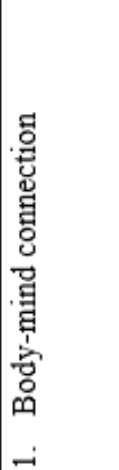 & 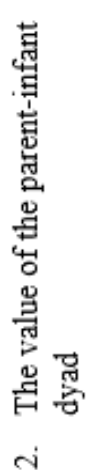 & 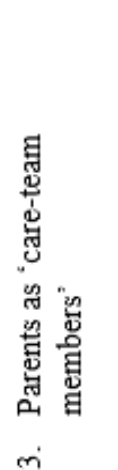 & 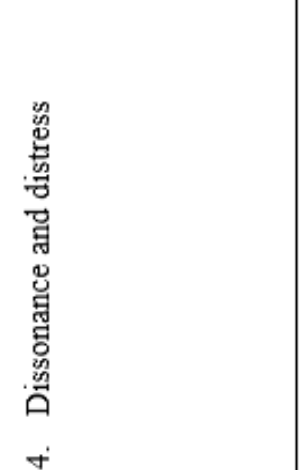 \\
\hline 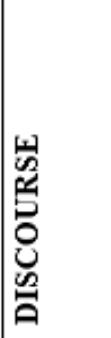 & \multicolumn{3}{|l|}{ 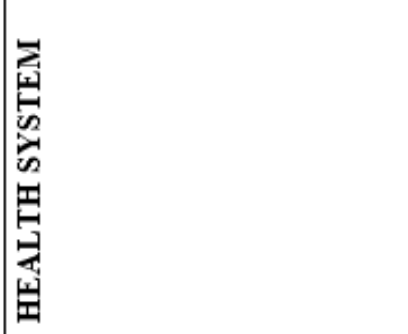 } & \multicolumn{4}{|l|}{ 梥 } \\
\hline
\end{tabular}


CHAPTER SIX: DISCOURSE ANALYSIS

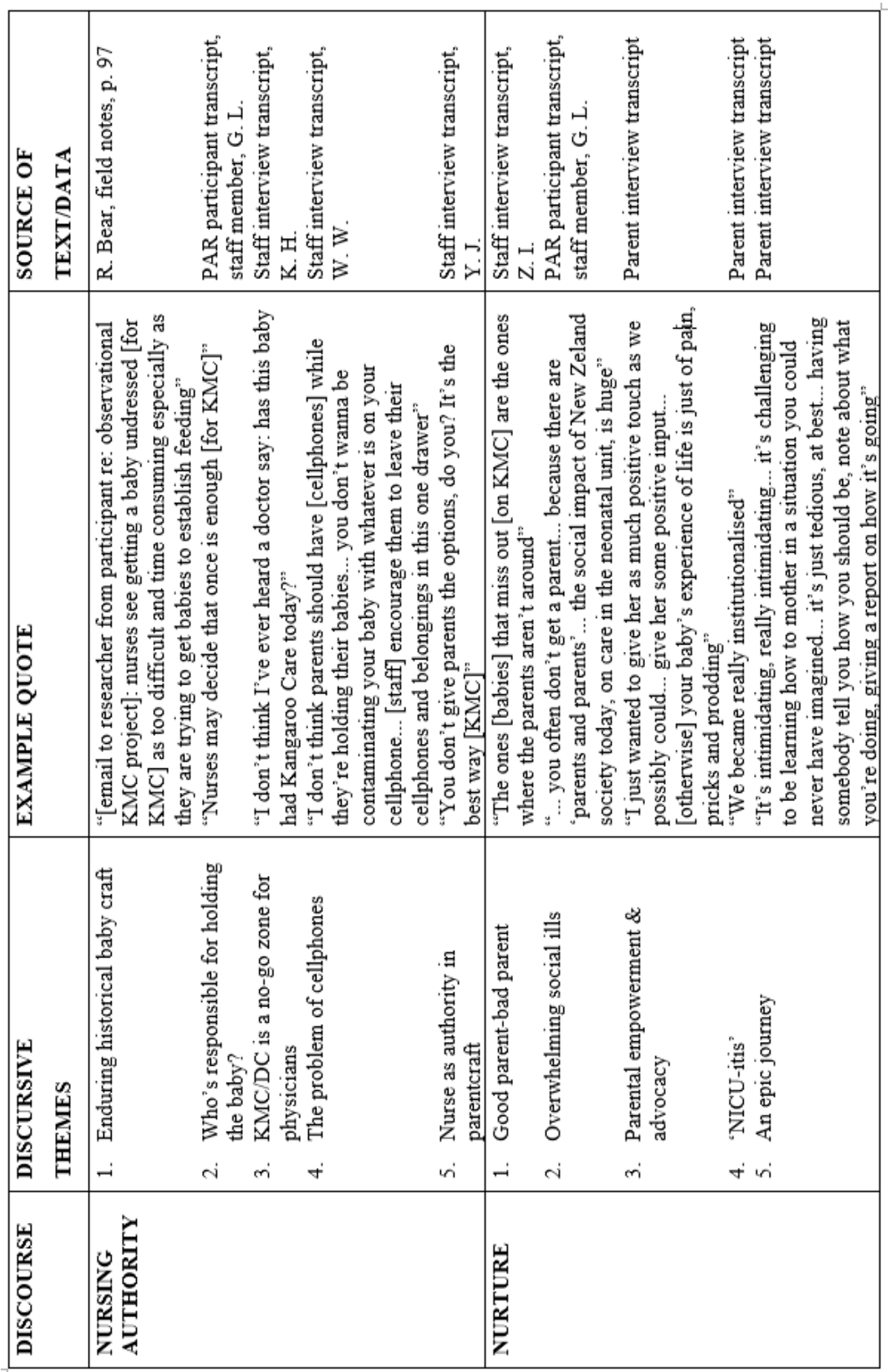




\section{CHAPTER SIX: DISCOURSE ANALYSIS}

The next sections discuss each of the five discourses including the discursive themes which emerged. Importantly, Foucault (1978) posited that there are an array of discourses operating simultaneously at any time, whereby (p. 100):

we must not imagine a world of discourse divided between accepted discourse and excluded discourse, or between the dominant discourse and the dominated one; but as a multiplicity of discursive elements that can come into play in various strategies.

Further to this description, concepts relating to $\mathrm{KMC}$ with this group are explored, those of 'absences and silences' and 'counter-narratives'. Discursive absence of $\mathrm{KMC}$ was a major finding from this analysis and ultimately informs the recommendations from this study.

\subsubsection{Biomedical technocratic discourse}

The technocratic discourse draws from Davis-Floyd's (2001) technocratic imperative that "stresses mind-body separation and sees the body as a machine" (p. S5) based on the highly medical nature of the intensive care environment. The major assumption applied to this discussion is that the hegemonic principle of the technocratic medical model (also called 'biomedicine'; 'orthodox', 'Western' and 'mainstream' medicine), revolves around the construction of safety, a phenomenon which the system itself has been simultaneously empowered to create, regulate and uphold through institutional mechanisms (Kallan, 2013). The first and most prominent theme from this analysis was indeed, 'safety-risk'. The technocratic medical model has an arguably dualistic history which has served to create a normative structure of safety which implies there is either safety or lack of safety; in other words, 'all or none' (Lapum et al., 2012). One effect of this dualism is the near-zero tolerance of risk, whereby beliefs, practices and/or actions which fall outside the normative assessment of 'safe' and low risk are polarised to a position of 'unsafe' and high risk.

The risk/safety theme was found in the case of this research to dominate all documentation and conversation examined for discourse. The word 'safety' and its many substituted euphemisms (stability, welfare, health, protection, and best interests) were extremely prevalent. Kangaroo Mother Care was framed as 'potentially' high risk, the assessment of which appeared to be overstated and out of context with respect to empirical knowledge and recommendations (Boundy et al., 2015; Conde-Agudelo \& Diaz-Rossello, 2016). Scholars warn that the omission of 


\section{CHAPTER SIX: DISCOURSE ANALYSIS}

'absolute risk' (the probability that the event will happen) in preference for the discourse of 'potential for risk' may indelibly influence carers away from an intervention through distortion of the probability of actual risk (Jordan \& Murphy, 2009). I identified the omission of an absolute risk discussion as a strategy which situated both staff and parents through the overstatement of 'potential' KMC risk. The effect of positioning KMC as one of 'potential risk' within the technocratic norm is to suggest that KMC may be unsafe and create an aversion to its use. The major 'power effect' of the risk/safety discourse is maintenance of control and compliance with the technocratic model of the NICU and undermining of true informed consent (Kadetz, 2014). This, in turn, positions patient/parents as passive receivers of biomedical knowledge, disempowered and unwilling to hold their full parental responsibility. 'Informed compliance' rather than fully informed consent, in accordance with the dominant ideology, is the outcome (Jordan \& Murphy, 2009). Consistent with the safety construct of 'acuity trumps care' is the popularised society-wide notion of 'ambulance at the bottom of the cliff'. This is a technocratic principle which leads to the prioritisation of short-term medical care of sick infants and the subjugation of developmental care: "Quality improvement is usually... more practical [than KMC] ... in terms of compliance with policy which minimises risk" (PAR participant transcript, NICU staff member).

The next theme, that of the 'technological imperative', is a phenomenon described in published literature as juxtaposed and problematic to the nurturing and caretaking impulse of a parent for their baby (Kallan, 2013). The hyper-valuation of reliance on machinery, constant monitoring and medical testing at the expense of the integration of more humanistic values has created a politicised and hierarchical organisation (technocracy) which relies on and creates the "subordination of individual needs to standardized institutional practices and routines" (Davis-Floyd, 2001, p. S7). An example of the 'technocratic impulse' was found when interviewing one mother of moderately preterm twins: "We were so thankful to be with the high-risk team... we just wanted to be sure... to do whatever it was that we could do... any intervention that would help us out".

Thirdly, the discursive theme of professional hierarchy and associated physiciancentrism was notable, also recognised in the established knowledge-base on the topic of how socioprofessional issues contribute to an undermining of public health: 


\section{CHAPTER SIX: DISCOURSE ANALYSIS}

"Because the Doctor said I should" (Wilson, 2012, p. 6). On this unit, medical rounds are considered a lynch-pin of workflow and daily organisation, in which doctors remain unwilling to commit to any consistency. I interpret this to be a discursive strategy which leaves parents as passive bystanders, uninformed and disruptive of their capacity to plan their daily activities, such as KMC. In addition to physician-centred medical rounds, case note documentation remains strategically in the realm of the biomedical, with parental input (or even access to) either actively or passively discouraged, represented by this staff comment: "Nobody (parents) should be reading (case) notes because they may not understand what is written" (Staff interview transcript, Y.R.).

Inextricably connected to the theme of professional hierarchy and physician-centrism is the paradigm of patriarchy and the paternalistic lens through which the ideology is operationalised. For the purposes of this discussion, the patriarchal paradigm is a socio-political system within modern society (including medicine), whereby the power is held predominantly by adult men. Also implicated is the resulting subjugation of women, children and the vulnerable, as well as the assignment of caregiving as a predominantly feminine trait and in healthcare, an activity which is subordinate to medicine (Kellett et al., 2014). One may consider medical paternalism, therefore, to be a tool of the patriarchy, whereby decisions are made on behalf of autonomous adults (if not by men, women within the patriarchal system), without full disclosure of known risks and benefits. The tool of paternalism is often used with the justification of being in the patient's best interests. I observed the potential for medical paternalism in statements from staff like this: "[Parent-led care] it can be frightening, some parents... without real understanding... their demands are usually egocentric... it could be quite dangerous" (PAR participant transcript, NICU employee).

The theme of scientification of medicine was expressed by multiple participants and within the documentation studied. This theme involves the ubiquitous assumption that medicine is solely based on quantitative (so-called, 'hard') science, extremely highly valued over other paradigms and affording most of the power within the healthcare domain. Not only are other 'ways of knowing' not well tolerated within the biomedical technocratic model, it is widely believed that the majority of medical decision-making is based on scientific evidence (evidence-based practice). This has 


\section{CHAPTER SIX: DISCOURSE ANALYSIS}

been shown within the medical and nursing literature, however, to be unfounded (Coughlin, 2014; Dogherty et al., 2013; Friesen-Storms et al., 2014; Greenhalgh et al., 2015; Grol \& Wensing, 2004). This, in turn, results in the use of 'opinion-based care', which whilst well-considered, is certainly not evidence-based, necessarily scientific or even qualified as such.

Related to the scientification of medicine is the discursive theme of medicalisation (and medical problematisation) of the postnatal period within the NICU. Nonessential obstetric interventionism has been shown more recently to be linked to increasing pre-term birth rates in developed countries and resulting increase of infant admissions to the NICU (Goldenberg, Culhane, Iams, \& Romero, 2008; MacDorman, Declercq, \& Zhang, 2010), the so-called "caregiver-initiated pathway to preterm delivery" (Frey \& Klebanoff, 2016, p. 71). The clearest examples of medicalisation of nurturing abilities within this NICU were associated with the mechanisation of feeding, dissuasion of extended parent-infant contact and discourse around 'proper' place of sleep/rest within cots and incubators: "To help them rest, small babies are nursed in incubators with a blanket over the top...” (Developmental care parent information document).

Lastly, the 'power of one' emerged as a discursive theme relating to the phenomena of gatekeepers and champions within the NICU. Within healthcare, the appointment of authority to individuals as gatekeepers and champions may be implicit, selfappointed and/or explicitly apportioned. The effect of this powerful position is to situate an individual as holding ultimate authority to halt and/or enable various operations within the system. Within this NICU, responsibility for the implementation of KMC largely fell on the attending bedside nurse. It was generally accepted that KMC practice was variable and dependent on the personality-based traits of each individual nurse: "there is so much variation in nursing styles and doctoring styles... you show up and you're not sure what their style is... what their opinion is... they might give you conflicting advice" (Parent interview transcript). Responsibility for KMC practice was quickly shifted to either a parent or a more senior staff member, often as a justification for negating its implementation.

Paradoxically, whilst physicians appeared to be given ultimate authority for KMC, they distanced themselves from the application of the intervention altogether. Resistance of gatekeepers to research and quality improvement to a more evidence- 


\section{CHAPTER SIX: DISCOURSE ANALYSIS}

based KMC protocol appeared to be used as a tool for maintaining personal preferences and the organisational status quo. In contrast, 'renegade' behaviour of champions was used as a tool to step outside of NICU standard practice, one nurse giving the example of enabling KMC more than once a day, aware that it was technically 'against policy'. Ultimately, the system of 'how $\mathrm{KMC}$ is done around here' was obscured by lack of clarity about exactly who or what is involved in its implementation, whilst proponents of the practice seek champions to support it and avoid prohibitive gatekeepers, where possible. In addition, organisational responsibility to improve on KMC education programmes for both staff and parents, may remain occluded due to the cultural acceptance of the role of gatekeepers and champions as being responsible for the practice.

\subsubsection{Health System Discourse}

'Chaos and crisis' was a prevalent theme penetrating into much of the activity of the NICU. There is widespread general discussion about the idea of chaos within the healthcare system, both in the societal domain and within academic discourse. As a result, reasons for the lack of translation of evidence into best practice is often cited as being due to the systemic duress being experienced within healthcare spaces: "realise we are resource poor... we do not meet best practice for minimum hours for nursing staff' (Staff interview transcript, G.L.). A funding theme was also highly visible, the constraints of which inevitably arose relating to the provision of basic, valued resources within the NICU: "[regarding funding of KMC] I love your idea that we might put some money into staffing this, but I don't think it will happen this year (laugh)!” (PAR participant, NICU staff member). In developing countries, scholars have identified increasing pressure on neonatal services in tertiary centres partly due to "rapid up-referral of patients, with limited down-referral of low-risk patients" (Feucht et al., 2016, p. 49), pointing to NICU overuse. This may be a factor in the New Zealand context of this study and has been suggested by observers of recent epidemiologic trends in other high-income contexts, such as the United States (Harrison \& Goodman, 2015).

Juxtaposed with the discursive theme of limited funding and associated requirement for healthcare cost-cutting, is the global health conversation relating to neoliberal power dynamics of 'for-profit' biomedicine (Ruiz et al., 2016). It is possible that technological interventionism is underpinned by neoliberal ideology, with 


\section{CHAPTER SIX: DISCOURSE ANALYSIS}

technology companies having strong agendas to teach healthcare professionals about the advantages of machine-based care in preference to human-based care. There is evidence that the power/knowledge constructs inherent within the healthcare industry influentially privilege the uptake of expensive technologies (for example, incubators), over simpler ones (such as KMC), for the purpose of profit generation (Abadia-Barrero, 2018; Syed et al., 2013; Syed et al., 2012). The theory exists that $\mathrm{KMC}$, a low-tech-low-cost-high-impact intervention, is situated within the healthcare and biotechnical industries in high-income countries as scientifically questionable and possibly even 'primitive'. Whilst evidence of neoliberal politics in biomedicine may be difficult to detect, it's possible that its effects are so deeply unconscious within this NICU community that reflection on discourse which represents this theme is not yet available. There are prolific examples from this context where technological interventionism is lauded as preferable to more humanistic care, and this, I believe, points to a discursive strategy of neoliberalism which affects KMC: "Premature babies are often "nested" in a roll of blankets inside their incubator to copy the restrictions found when inside the womb. This comforts small babies... allowing your baby to rest for long periods without disturbing them" (Developmental care parent/caregiver information document).

\subsubsection{Humanism}

A humanistic discourse was discernible within this NICU's culture, albeit a minority category in comparison to medical technocracy. In the field of medicine, humanists are advocating for medical system reform through the application of a more loving model based on "relational, partnership-orientated, individually responsive, and compassionate" (Davis-Floyd, 2001, p. S10).

The first of the four humanistic themes I have called 'body-mind connection', the discourse of which acknowledges that human physiology is connected to mental health, with the awareness that environmental effects of NICU hospitalisation may constitute a trauma. Body-mind connection discourse contributes to situating babies as humans with their own personhood and whole system health to consider. The discursive effect is to acknowledge each individual as a social person with biological and emotional needs to value - one of the basic tenets of 'the caring professions' and a principle of basic infant human rights (World Association for Infant Mental Health, 2016). Concerns for the ongoing effects of poor infant mental health, due to 
suboptimal care of babies, were present for both staff and parents, reflected in this quote by one staff member (PAR participant interview, G.L.):

[the topic of] infant mental health is starting to grow, um, its voice louder, thank God... I would love to have someone focus on infant mental health on the staff... minimise some of the trauma... if we can't put a central person in there who only ever gives them positive touch and says "hang on there, we'll get through this together, you're safe, I love you"... if we can't do that, it's really scary.

The importance of the parent-infant dyad was a humanistic theme, discussed by staff, parent and through organisational documentation: "I just love seeing how relaxed the parents are [in $\mathrm{KMC}]$... it's just their little baby-box zone" (Staff interview transcript, W.M.). This quote represents the discursive strategy of lay language use which supports the dyad using 'soft' and caring communication. This positions the parents as their babies' primary and irreplaceable caregiver, increasing their confidence for the desirable discursive effect of zero-separation of babies from their parents (Altimier \& Phillips, 2016). A shift in caregiving roles requires that staff release absolute control (ownership) of the infants' care in favour of true partnership with families. Paradoxically, one notable undesirable effect of the use of lay language (such as 'cuddle'), within the dominant technocratic paradigm, is to devalue the intervention to the medical fraternity through its representation as nonscientific, non-medical and/or 'fluffy' (PAR participant transcript, NICU employee):

[Regarding KMC-associated language] I think "cuddle" immediately means "that's fluffy" and it's "nice to have, not a need to have" ... perhaps we should call it "kangaroo care technology" ... I'd put "essential" in... should it be "skin-to-skin essential intervention"?

Next, the relationship-based theme of 'parents as care-team members' was visible within the discourse and represented as an ideal: "Confirm that the parent wants to participate in this activity... discuss intended benefits and potential risks... explain the procedure (KMC)" (Kangaroo Care Staff Guideline). The potential effect of a shift in responsibility for infant care from nurses to parents could be to position nurses as coaches and facilitators rather than primary caregivers. Interestingly, my observation on this unit was whilst medical staff retain the ultimate power to withdraw KMC practice, there was a simultaneous discourse around the practice being 'outside their scope'- a clear contradiction. Through feminist and Foucauldian lenses, organisational gender and professional-based power imbalances were apparent. My conclusion on this point relates to the discursive strategy of blame: the inconsistent application of KMC was apportioned to nurses (too busy, unmotivated, 
overworked); and/or parents were blamed for being unavailable, not confident and not safe. This blaming strategy diverted attention from addressing the systemic issues of the NICU, which were arguably far more powerful than any of these individuals in the standard of KMC care.

The final theme of 'dissonance and distress' emerged in the humanistic category, with specific reference to a lack of developmental care, including KMC. The acknowledgement of the importance of humanistic care methods and frameworks was visible within the discourse from managers, the developmental care special interest group and within guideline and policy. In actuality, the implementation of developmental care, particularly with regards to Kangaroo Mother Care, remains outside of the normative structure, raising questions about the agendas of the NICU social system in this regard. A major effect of this ambiguity around DC and KMC may be value dissonance and moral distress for individuals who are aware of the potential for improved care and health outcomes with the use of these modalities, whilst not having the power to systematically embed them (PAR participant transcript, NICU staff member):

... a lot of the care for neonates is around intensive care... and because we're so focused on that and much to my distress in many ways, the developmental care has almost been sidelined... "oh yes, been there, done that, give the baby some boundaries"... rather than saying hey... doing things that we've talked about are going to make things better forever, not just this week.

\subsubsection{Nursing authority}

Nursing authority constituted a major discourse in the data. Significant knowledge/power influence on the care provided to babies and families on the unit emerged throughout the discourse analysis. Four discursive themes, including their positioning effects on NICU members and the broader effects of the discourse on NICU culture, are described.

Enduring historical 'babycraft', and who was positioned to be control of it, was the most prominent theme relating to non-medical aspects of care, with lasting characteristics of 20th century infant care-giving being extremely pronounced.

Whether the baby was receiving intensive medical care, or one of the more than $70 \%$ of babies simply requiring nurturing support to feed, sleep and grow, nursery-based care remained highly scheduled and a major factor in maternal-infant separation. Discourse relating to patterns of care centred on three-four hourly feeding, with the 
expectation of limited time at the breast and assumed milk top-ups; cot-based sleeping with an overarching 'back to sleep' rhetoric and implication that handling tires and stresses infants; and the avoidance of excessive stimulation through too much cuddling, handling or time out of their beds (Staff interview transcript, K.H.):

They're not getting real optimum sleep when they're being held by you [the parent] ... feed and put back [into cot/incubator] ... let them sleep, let them grow.

Parents were positioned as risk-taking if their baby slept anywhere other than in their incubator or cot, with ongoing reinforcement through staff conversation and documentation that 'other' sleeping was disruptive. Disrupted sleep, in turn, was associated with poor growth and development and associated in this unit with overhandling of babies by their parents.

Nursing authority also had the discursive effect of placing infant feeding in the medical - reductionist and mechanistic - discourse focusing on volumes of milk and frequency of expression. There was an absence of discussion about the quality of the breastfeeding relationship and associated bonding processes. The term 'top-up' was arguably the most common one used in care discussions for babies within the predischarge phase of hospitalisation: "It was just so clinical... give your breastfeed a code, how many mls [millilitres] you're gonna give as a top-up, what was the nappy like... that was difficult" (Parent participant transcript). Combined with the notion that the baby should feed quickly to avoid overtiring, a history of poor growth, and needs to be back in bed as soon as possible, the cumulative effect of nursery routines produces a 'hands-off' parental approach to nurturing their infant. As a result, mechanised feeding, cot-based sleeping and detached, 'hands-off' care is likely to interrupt KMC, breastfeeding and bonding and increase negative psychosocial indices.

The theme of 'who's holding the baby' presences the question of where the responsibility lies on the continuum of babies' care. At one end of this continuum, nurse ownership discursively positions parents as powerless, and babies as patients. I was told in general conversation with NICU staff at least four times, for example, that they were pretty sure that parents weren't doing KMC when they got home after discharge and therefore (quite pragmatically), what was the point of doing it on the unit? Similarly, parents were implicitly blamed for not using KMC as a care method in pre-discharge rooms, based on, as one staff commented 'Once they're in cots, the 


\section{CHAPTER SIX: DISCOURSE ANALYSIS}

parents should be doing everything" (Staff interview transcript, E.W.). This comment is categorically over-simplistic, given the continuing interdependence of nurse and parent, even at this stage of hospitalisation. For example, there was implication of 'nurse ownership' and the ongoing requirement for partnership within parent information documentation stating: "Kangaroo Care - holding your baby next to your chest skin-to-skin is recommended. Your baby's nurse will assist you with this" (Parenting your preterm baby 36-40 weeks gestation). Nurse-led authoritative knowledge and resulting possessive practice were also plainly present in this comment by one experienced neonatal nurse, recounted by me in field notes $(\mathrm{R}$. Bear, field notes, 12.05.2017, pp. 115-116):

our nurse of the day is gruff and announces loudly to me when the twins' mother leaves the room, that she is 'happy to play bad cop'... this mother holds these two far too much... they need to get used to their beds.

Using Foucauldian principles to examine the continuum of caregiver responsibility for babies, the over-simplified view of full parental authority for their babies' care negates the power relations inherent in discursive practices of $\mathrm{KMC}$, nursing and parenting. For example, the information available to parents of pre-discharge infants was largely devoid of the concept of KMC, and it was also absent from predischarge room discourse. Nevertheless, nurses still described the responsibility for $\mathrm{KMC}$ as lying exclusively with the parent, clearly outside the implicit expectations of the unit. The discursive effect for parents was that KMC was positioned as no longer appropriate, required and/or valid at this stage of hospitalisation. Such discursive absences were subtle, yet as informing and reinforcing as the prevailing and normative discourse.

The theme of $\mathrm{KMC} /$ developmental care as a 'physician no-go-zone' was commonly represented within the discourse data. It was repeatedly expressed that physicians and other medical staff distance themselves from the frontline implementation of $\mathrm{KMC}$, using the discursive strategy of classifying the intervention as 'nurse-led' and therefore abdicating responsibility for engaging with it. All interviewed participants maintained that non-medical care of infants was outside the scope of practice for physicians. Whilst objectifying KMC as a psychosocial intervention and situating nurses as leaders of it, it was also clear that physicians held the ultimate authority for the type of 'cares' a baby could receive, possessing the power to disallow KMC if they saw fit. In this way, the historical positioning of paediatrician-as-expert for 
infant care continues, having the discursive effect within the NICU of undervaluing $\mathrm{KMC}$ to both parents and staff, as well as diminishing its role within the day-to-day care of infants: "None of our consultants, I would say, have an interest in developmental care really... KMC is sometimes seen as the "fluffy stuff'" (Staff interview transcript, R.N.A.).

The final discursive theme of 'the problem with cellphones' was surprisingly intense within the staff discourse of infant care. Cellphone use by both staff and parents was viewed as problematic for different reasons. For staff, the modelling of cellphone use and the inevitable loud talking and noise pollution that results, situated staff as being part of an 'environmental issue': "there is reluctance of practitioners and patients to leave cellphones out of the unit... rounds are noisy, people speak too loud and 'quiet time' is no longer implemented" (R. Bear, field notes, 14.06.2016, p. 44). A different discursive strategy existed for parents with cellphones, positioning parents as emotionally unavailable to their infants through the discourse of paternalism. The discursive effect was to objectify cellphones in a way that staff felt entitled to control parental behaviour, justifying either withholding or being less proactive about KMC:

"[Some nurses may decide that] once is enough [for $\mathrm{KMC}]$... with a lot of our younger mothers, it's the damn cellphone [which disrupts]" (PAR participant transcript, NICU Staff member). In response to the notable absence of KMC practice with pre-discharge babies, largely through nursing authority discourse, I summed up my observation in this way: "caring is kind and considerate, yet poorly focused. [It is] not focused on the biological needs of babies and instead, models an oldfashioned nursery" (R. Bear, field notes, 27.05.17, p. 133).

\subsubsection{Nurture discourse}

Society-wide discourse relating to parental nurture of babies constitutes NICUexperience as an 'epic journey' for parents (Aagaard \& Hall, 2008; Black, HolditchDavis, \& Miles, 2009; Davis et al., 2003; Gondwe \& Holditch-Davis, 2015; Kallan, 2013; Maghaireh et al., 2016). The theme of 'epic journey' also emerged from this analysis. Discourse from the one parent-participant from this study confirmed the arduous and mentally challenging experience of being a long-stay parent on the unit. Narrative from ' $\mathrm{K}$ ', NICU-parent, revealed many of the complexities presented within the current literature describing maternal identity and role attainment in the NICU (Aagaard \& Hall, 2008). For example, she was overwhelmed, intimidated and 


\section{CHAPTER SIX: DISCOURSE ANALYSIS}

felt out of place within the environment and identified her own struggle to transition to parenting medically fragile babies under the 'medical gaze'. K also felt simultaneously grateful for nursing support whilst resentful of a lack of privacy and feelings of being intruded upon by the same nurses, indicative of her lack of power to control her own circumstances.

Relating specifically to $\mathrm{KMC}$, K revealed complex emotions relating to $\mathrm{KMC}$ which would arise intermittently and create some distress. As far as I'm aware, these have not previously been reported within the NICU/KMC literature (Parent interview transcript):

[KMC] was often very pleasurable, but just not $100 \%$ of the time... something interesting about KMC... challenging for me... I would often have an unwanted thought, the sensation when I was holding them of just really being trapped... to jump up from the chair and, you know, throw them down.

The complexity and, at times, internal conflict which this one woman felt about $\mathrm{KMC}$ was entirely new to me, not having read about it within the literature, or personally experienced anything similar within my own NICU-parent journey. This parent's relationship to $\mathrm{KMC}$, although mostly positive, was certainly complex, the understanding of which may offer some insight for other parents experiencing similar conflict.

A 'good-parent-bad-parent' dichotomy was present in the discourse from both staff and parent and likely to also be constituted by society's moral discourse around appropriate parenting and nurture: “... you often don't get a parent... because there are "parents and parents"” (PAR participant transcript, NICU staff member). There was obvious parental struggle with the nurture discourse, our participant describing her attempts to make sense of emotional difficulty during KMC: "I wondered how common it was [unwanted thoughts during $\mathrm{KMC}$ ], you'd feel very ashamed and you'd think, 'I must not be a very good mother'" (Parent interview transcript). Staff discourse constituting parents as 'good' or 'bad' within this unit centred largely on the construct of the 'social ills' of the heterogeneous group for whom they cared. A 'good parent', therefore, implied a 'social fitness' to nurture their infant. Conversely, a 'bad parent' was deemed 'socially unfit' and positioned as 'high risk' with respect to caring for their baby, particularly relating to the intervention of KMC. A possible effect of this sort of dualistic, institutionalised classism was paternalistic decisionmaking that withholds knowledge and care from those who become labelled as 


\section{CHAPTER SIX: DISCOURSE ANALYSIS}

socially unfit (Landzelius, 2006). The discursive effect is further separation of parent and child, inadequate bonding, poor parent-practitioner partnerships and a perpetuating cycle of family inability to attach and assimilate the baby within their family unit. Normative NICU-based routines and values are used to compare parents who nurture their babies in ways that fall outside of the dominant paradigm.

Evidence was found on this NICU of parents being labelled as disruptive to their babies' rest (and therefore growth and brain development) if they provided KMC for 'too long' - an arbitrary number that was likely to be more than a few hours - and negligent if they didn't turn up to nurture their babies in the way that was deemed appropriate by normative practice and policy. The positioning of parents (most frequently, the mother) as good or bad appeared to be an unconscious strategy by NICU staff and the organisation to adjust the care that they gave to the individual, whilst also applying pressure on parents to conform to the normative patterns of care - an example of Foucault's (1977) disciplinary power. In this case, the response of parents was to adjust their own behaviour by internalising the normative structures, using the Foucauldian concept of "self-regulation" (Griscti et al., 2016, p. 243). The effect of this phenomenon is to 'keep the peace' and ensure good working relationships with staff, in the hope that their babies continue to receive appropriate care. One discursive effect of self-regulation by parents is to unwittingly strengthen the authority of the healthcare professionals and institution.

Analysis of staff discourse revealed the theme of 'overwhelming social ills', expressed strongly by some NICU employees, that the issues of 'social unfitness' (and therefore insufficient nurturing) were almost overwhelming within this unit (PAR participant transcript, NICU staff member):

The social impact of New Zealand society today, on care in the neonatal unit is huge... either the antenatal environment, CYFs [Child, Youth and Family] identification of the parents' inability to cope... impacts of drugs or violence or poverty... it's really difficult when half the mothers couldn't give a damn [apologises about the expletive]... half might be an exaggeration...[nurses] sometimes forget they have... normal parents who really want to know about care. They become the good parents.

The positioning of parents as unsafe, unfit, a cause of stress, a nuisance, incompetent and/or under-confident, had the effect of persistent powerlessness for some parents and difficulty gaining autonomy in their primary caregiving role. 


\section{CHAPTER SIX: DISCOURSE ANALYSIS}

The next discursive theme was parent 'NICU-itis', identified by both staff and parent, referring to a passive state of parents, whereby they stand back and allow staff to do for their babies what they could potentially do for themselves. Tension existed for the participating parent between the nursing and medical support they required and the independence that they desired, the inner-conflict being resolved mostly by the choice to take the path of least resistance - passivity. Subsequent to immersion within the NICU discourse, I surmise that the origins of NICU-itis are at least partly through the objectification of NICU-parenting as 'somebody other than the parents' work'. This effect may be through the discursive practices of institutionalisation, medicalisation and/or scientification of nurture and the multiple power dynamics which result in a diminished identity of the parent in the primary caregiving role (Parent participant transcript):

[teaching of infant care] it's pretty informal... taught by whatever nurse is on duty... we got some really experienced nurses to tell us, "this is how they like to be touched" and "this is a good way to interact with them"

The overall discursive effect of biomedical and nursing authority discourse was that nurses retained the capacity to decide when, where and how nurture took place, including KMC. This was through the subjugation of non-medical practices to the routines and requirements of the medical, positioned through authoritative biomedical and technocratic discourse.

Subjugation of parental authority within the NICU setting was juxtaposed with 'parental empowerment and advocacy'. This theme was partly constituted through global healthcare discourse calling for a shift of control from physician-centred to parent/patient decision-making. I suggest that whilst the shift appears to be supported in theory, there was a power-resistance network inherent within discursive practices that position KMC. Inquiry into KMC revealed a "plurality of struggles... [that] attest to multiple sites of power and resistance" (Douche, 2007, pp. 84-85). There was significant discordance between some staff perspectives and the parental empowerment discourse present in the education-based documentation sourced for this analysis, due to fears of parental competencies and patient safety (Bourque et al., 2017; Weiss, Barg, Black, \& Joffe, 2016). I suggest that KMC educational policies on this unit were borne out of nursing authority discourse - making claims about their professional knowledge to secure control over their practice (Papps \& Olsen, 1997). On this theme, the participating parent appeared to reach a hard-won stage of 
empowerment that suited her and her family, whilst staff discourse reflected the perspective that many parents remain overly reliant on nursing care of their infants until their discharge.

Most of the observed discussion and discourse gleaned from the data reveals that in this setting, 'nurse as authority in parentcraft' remained the dominating and normative structure. Due to a combination of parental ambiguity about their caring role and persistent powerlessness, nurses were positioned as authorities in parentcraft, in turn serving the current institutional status quo. The discursive effect was that power relations were in place that served the NICU-system, rather than the babies and parents for whom they were intended to care. True parental partnership, therefore, eventuated for only a select few and remained elusive as a cultural norm for most families for the majority of their stay.

The five discourses emerging from this analysis have been described and constitute the NICU as a 'heterodiscursive space' (Springer \& Clinton, 2015), with the most notable presence of a technocratic model of biomedicine within the NICU, broader health system effects on KMC, a small but significant humanistic discourse and the discourses around nursing authority and nurture. Next, the following two sections describe less prominent, yet equally important aspects of any Foucauldian-informed discourse analysis, those of absences and silences and counter-discourses and resistances. It is in the final examination of these two aspects of discourse that the over-riding representation of the topic of Kangaroo Mother Care within this unit is revealed, providing a basis for the proposed development of the programme.

\subsection{DISCURSIVE ABSENCES AND SILENCES}

Through a Foucauldian lens, the discursive silences constituting the social construction of $\mathrm{KMC}$ are as informing as the visible and predominating discourses (Carabine in Wetherell et al., 2001). I discuss these here with reference to aspects of the NICU environment, policy and guideline documentation, and community conversation. Many factors contribute to poor clarity and presence of the KMC programme on this unit, including a lack of an overarching $\mathrm{KMC}$ vision and low prioritisation at organisational and individual levels. Here, I re-presence my purpose for performing a discourse analysis within this PAR-led research. It was to examine 


\section{CHAPTER SIX: DISCOURSE ANALYSIS}

the power/knowledge relations constituting the established institutional culture on this NICU, influencing KMC practices and exerting discursive effects on its subjects.

Upon arrival on this NICU as a KMC researcher, the first thing I noticed was the visual absence of KMC. If healthcare leaders and practitioners have the intention to expose its community to the evidence-based practice of $\mathrm{KMC}$, low levels of promotion in their environment are both causative and a result of low levels of KMC activity in their space. For example, within the three pre-discharge areas, including the main corridors, there was just one small A3 sized poster of a KMC-dyad, which was poorly positioned in one room and difficult to see. Ideally, there would also be a purpose-prepared area for information on parent-led care interventions, such as $\mathrm{KMC}$ (for example, KMC parent guideline, developmental care parent guideline), which parents could be directed to for educational material. This would be indicative of a coherent and focused education programme containing appropriate materials: "It's a bit haphazard, the utilisation of handouts [parent education] and that sort of thing" (Staff interview transcript, Y.J.). Anecdotally, this unit had previously provided the space for parent education materials, yet this had lapsed some time before and no longer existed. On the matter of staff education, new staff members receive just one hour of explicit KMC training when they are orientated on the unit, with one nurse noting: "no-one actually teaches the importance of $\mathrm{K}(\mathrm{M}) \mathrm{C}$ to the staff... we really need to address the gap in education of the staff' (Staff interview transcript, R.N.A.).

Two further notable absences apply to observation charts and case note documentation. Firstly, there was no allocated space or prompt for KMC recordings amongst the myriad of required observations; and secondly the term 'Kangaroo Mother Care' was often reduced to the term 'cuddle' or 'kangaroo cuddle', which, I argue, results in the positioning of the intervention as psychosocial 'fluff' and outside the realm of the normative paradigm - biomedicine. During the final interview with our key stakeholder, we discussed the necessary addition of KMC 'prompts' to the observational charts. This would have a two-fold effect of reminding new and task-focused medical staff to discuss and implement KMC with the parents and secondly, raise the awareness of it with other community members (PAR participant, NICU staff member): 
So if you've got a space there [in the chart] called 'Kangaroo Care', it's going to trigger a nurse to think "oh, there hasn't been any for several days, have a conversation" ... a Kangaroo care tick box of some sort

As mentioned by one staff member in interview, raising the profile of KMC to an essential intervention through the use of appropriate language may have the effect of reminding and re-focusing staff and parents of its importance: "[KMC associated language] I think 'cuddle' immediately means 'that's fluffy'... should it be 'skin-toskin essential intervention'?" (PAR participant, NICU staff member).

The complete absence of KMC discourse and practice within the main pre-discharge room is a most notable finding from this research. Whilst framed as the parent's responsibility for care decisions within the latter stages of their hospitalisation, the lack of support for KMC with this group, combined with the tendency for the practice to cease to be encouraged by the staff once babies are dressed and in cots, appear to be the most influential factors resulting in KMC absence. Obvious missed opportunities for babies to experience $\mathrm{KMC}$ were when babies were already undressed, especially for either bathing or weighing. Although this situation presented itself seven times during my observation, there was no conversation by either nurse or parent to suggest awareness of the opportunity for KMC at this time.

Referencing the technocratic/humanistic/holistic framework presented in table 10, the holistic medical paradigm was completely absent from NICU discourse in the data, with no direct discussion to point to why this may be. Medical holism, for the purposes of this discussion, is the awareness and integration of the body and mind as a united system, seen within a whole-life context, considering inter-relationships of whanau/family, society and the environment (Davis-Floyd, 2001). Healing is believed to happen from the 'inside-out' as an ongoing process; and care is individualised on the basis that multiple knowledges extend from and encompass the physical, emotional, mental and spiritual realms of each person (Ayers-Gould, 2000; Davis-Floyd, 2001; Garcia \& Yim, 2017). Setting it apart from the concept of 'humanistic', holistic medicine is underpinned by philosophies of spirituality and unity consciousness, not generally considered by the two other medical models: technocracy with its focus on the physical and body-as-machine; humanistic with its focus on the biopsychosocial and emotional (Ayers-Gould, 2000; Davis-Floyd, 2001). A predominating technocratic model is likely to be dissuasive of holism due to its inherent characteristic of dualistic intolerance and marginalisation of other 


\section{CHAPTER SIX: DISCOURSE ANALYSIS}

healing modalities and values (Davis-Floyd, 2001). In addition, technocracy's high valuation of quantitative science and risk/safety discourse further silence the minority 'voice' of holism, a pattern seen widely in Western, secular society.

A recent discursive appraisal of KMC relevant to the notion of holism was directed to the World Health Organisation by KMC scholar Abadia-Barrero (2018) in his highly political paper on the deterioration of the original Columbian KMC programme (p. 23):

It seems that the WHO is using accepted scientific guidelines as a substitute for KMC principles... a way to avoid discussions regarding the importance of love, the meaning of warmth, or the dangers of commodifying health care.

The discursive absence of holistic discussion on this unit, like that of the World Health Organisation (2003) guidelines, may be considered a discursive strategy, stating that there is no place for holistic language in the healthcare environment. In turn, individuals with holistic views and practices are positioned as 'other' with the effect of dehumanisation of the individual through the diminishment of their perspectives (Ayers-Gould, 2000). I did not find love, in the form of KMC, within this unit. The integration of holistic principles with both technocratic and humanistic discourse, through the acknowledgement of the whole-person's body, mind and spirit, may enhance community-members' wellbeing, including that of the hospitalised infant (Baum, Weidberg, Osher, \& Kohelet, 2012).

The final and perhaps most influential discursive absence affecting $\mathrm{KMC}$ on this unit was the physicians' omission to engage with the integration of KMC into their discourse and scope of practice. In addition, the technocratic medical model is supported by organisational and societal influences (including historical patriarchal policy and practice), to largely discount psychosocial and spiritual aspects of humanity. Through a Foucauldian lens, the predominating technocratic and biomedical discourses on this unit were normative. The resulting power relations positioned frontline staff and consumer subjects involved with KMC as less knowledgeable than the authoritative biomedical 'voice'. The status quo of hierarchical biomedical authority within NICU discourse objectified Kangaroo Mother Care as an intervention that is 'nice to have, not need to have'. Various discursive strategies, at multiple levels of the NICU community, resulted in a poorly 
functioning KMC programme that was near-absent from the view of those who held most of the power to effect changing it.

\subsection{COUNTER-DISCOURSES AND RESISTANCES}

Throughout the evolution of his thinking and writing, Foucault (1978) continued to describe that "where there is power, there is resistance" (p. 95). Whilst the constitution of normative discourse as 'truth' is powerful and pervasive, the mechanisms through which persons uphold their own agency may be observed through counter-discourses (Carabine, 2001; Douche, 2007). Referring to the nonbinary nature of power relations, Douche (2007) asserted that "The mutuality of power-resistance is not ensnared within a binary opposition, between those in power and those who are not" (Douche, 2007, p. 84). KMC-related counter discourses were found in the data from this study with the effect of resisting the normative status quo. Whilst they represented a small minority within the language and texts, three emergent themes were described: 'the rebel nurse'; 'show me the money'; and 'where is the partnership?'

An individual nurse, positioning herself as 'resistor' of the status quo, described a limited behavioural repertoire of practising what she believed to be morally 'right' in the face of institutional opposition. Discourse around morality from the rebel nurse was one example of resistance to normative KMC practice. She spoke of openly flouting the standard recommendations despite awareness of the disapproval of her practice. In a sense, the rebel nurse could be viewed as a champion who wasn't designated their power by the institution, they simply claim it for themselves. Whilst this approach was deemed professionally high risk due to the threat of horizontal and organisational sanction, it had the effect of modelling to others that there are opportunities to create change through personal action. The nurse involved was willing to support and encourage parents to KMC more than once a day, for as long as they desired, openly disagreeing with the current implementation of the practice (Staff interview transcript, E.W.):

I don't know where the one hour [KMC] came from, I really don't... I don't think its done well enough on this unit; we're too restrictive... why can't the grandparents be involved [with $\mathrm{KMC}$ ]?

In doing so, she modelled the values of individualised care based on evidence-based knowledge, despite the norm on the unit. 


\section{CHAPTER SIX: DISCOURSE ANALYSIS}

The discursive theme of 'show me the money' revealed a resistance to the normative organisational discourse of limited resources and lack of funding for appropriate KMC and developmental care. Participants described with righteous anger the lack of resources for items of basic care of mothers and families with very complex health and social needs. I was impressed by the futility of one participant, describing her desire to institute a 'cuddle aunt' programme for babies who were receiving very low levels of positive touch. It had thus-far proved impossible to secure low-level funding for the humanistic intervention, despite the likelihood of improved maternalinfant developmental outcomes. Drawing on the empirical knowledge base for KMC, I similarly questioned why a KMC programme, characterised as 'low-cost/lowtech/high-impact', and in line with World Health Organisation recommendations for quality of care, could be overlooked and justified by insufficient funding (ten Ham, Minnie, \& van der Walt, 2016; World Health Organization, 2006, 2015). Whilst multiple financial barriers to KMC have been identified within the literature, I contest the validity of cost being a valid prohibitive factor for the intervention. In agreement with other KMC scholars of recent times, I conclude that the discourse around funding must in some way be political in nature (Abadia-Barrero, 2018; Charpak \& Ruiz, 2016; Uwaezuoke, 2017).

Lastly, 'where is the partnership' emerged as a counter-discourse from several of the participants. This theme implies incongruence between the spoken ideal of partnership and its operationalisation with parents on the unit, largely through the discourse of nursing authority. The objectification of partnership as an ideal had resulted in a discursive construct that staff and consumers were both questioning in the face of enduring hierarchical structure: "we give lip service to partnership with parents... I'd like to see us actually exploring what partnership means" (PAR participant, NICU staff member). The absence of explicit reference to parental partnership relating to KMC and DC within the documents I examined, add weight to the lack of vision and/or intent to enable true collaboration and power-sharing with parents. This theme had clearly been identified by the community and appears to be actively imbued in a counter-discourse for the purpose of effecting change.

\subsection{SUMMARY}

Prior to the application of the discourse analysis, there was an unexplained ambiguity and dissonance relating to the KMC programme reflected by two main 


\section{CHAPTER SIX: DISCOURSE ANALYSIS}

factors. Firstly, a know-do gap, and secondly, an unrealistic community belief that KMC was well established and functioning. The primary analyses provided little evidence to explain why these occured. Two major points will be addressed to conclude this chapter. Firstly, I describe how the addition of a Foucauldian discourse analysis met the research questions identified in the introduction. Secondly, I address how the process of this analysis was beneficial for exploring the phenomenon of ambiguity and dissonance between 'what was said and what was done' with regards to KMC on this unit, the so-called 'know-do gap'.

The analysis found five major discourses, from which multiple discursive themes were considered. Power relations that affected KMC through various discursive strategies were described. The humanistic intervention of KMC had been objectified as 'fluffy' and positioned outside of the normative paradigm within the NICU's biomedical technocracy, with the effect of marginalising the babies' perspective and reducing parental authority and voice. Beneath the umbrella of a participatory action research approach, I undertook the discourse analysis to make a more in-depth inquiry into the previously unexamined power/knowledge constructs in one NICU. The purpose was to raise awareness of the participants and members to the effects of organisational and systemic discourse on KMC. Many of the discourse findings were corroborated in discussion with the final PAR participant involved with the research.

The use of discourse analysis, informed by Foucault and feminist theories, enabled an inquiry into power relations that constitute which knowledges were priviledged within this NICU. The hegemonic biomedical technocracy was found to position parent/patients as outsiders (or worse, stressors of their infant) with little authority or autonomy to make decisions about how they nurtured their infants. At the top of the hierarchical pyramid, physicians were imbued with ultimate authority, whilst at the bedside, nurses held authoritative knowledge and therefore, power. The discursive effect was to subjugate parental authority for the nurture and care of their babies (Altman et al., 2014). Discernably a strategy of a patriarchal paradigm, the principles of the technocratic model produce power differentials and effects through discursive constructions of safety, risk, 'expertise', and the devaluation of alternative forms of knowing (Davis-Floyd, 2001; Jordan \& Murphy, 2009; Kadetz, 2014). Lastly, the low-cost, biopsychosocial intervention of Kangaroo Mother Care, itself, has been situated in the developed worlds as a poor alternative to incubator-based care 


\section{CHAPTER SIX: DISCOURSE ANALYSIS}

through the mechanisms of the technological imperative and neoliberal biomedicine. Pointedly, $\mathrm{KMC}$ is inconsistent with "profit-generating principles of the reverse innovation business model" (Abadia-Barrero, 2018, p. 6), and therefore not a technology or innovation that may be co-opted to turn profit.

Based on these findings, it is my conclusion and assertion that the normative paradigm within the NICU environment remains predominantly under the "distinct cultural authority" (Davis, 2016, p. 61) of biomedicine. I found that the default setting of incubator-based care was maintained by individuals at various levels of NICU community involvement including parents, staff, management and administration. This analysis has identified paradigmatic aspects of NICU culture that largely prevent the implementation of Kangaroo Mother Care within this setting. The embodiment of a consistent and evidence-based KMC programme requires the integration of humanistic and holistic medical paradigms at a systems level that may be absent or at least in their infancy for this NICU community. The constellation of predominating technocratic biomedical care, nursing authority and lack of parental empowerment created a paradigm of caregiving that resulted in the inconsistent application of Kangaroo Mother Care.

This analysis has contributed evidence about power relations, expressed through discourse, that serve as barriers to KMC quality improvement. The seventh and final chapter brings together the key findings and recommendations that have emerged from this research, as well as the contributions it claims toward KMC and methodological knowledges. 


\section{SECTION C}

\section{CHAPTER SEVEN: DISCUSSION}

This chapter concludes my study, firstly by summarising the research issues, aims and objectives. Then, the key findings from all five phases of the study, local and global NICU-narratives, my own NICU-story, and the secondary discourse analysis are discussed in the context of what contribution they make to the body of knowledge for Kangaroo Mother Care within the NICU environment. The contributions of PAR methodology are reflected upon, including the most significant challenges to its use, the real-time adaptations that were made, and the limitations of the approach. Implications of this study for policy and practice of KMC in the NICU environment, both specific to this unit and more globally, are described. Specific recommendations are made to the NICU participants involved with this research, followed by broader considerations relating to the adaptability of PAR for the improvement of KMC in other contexts and for future research.

Empirical evidence is unequivocal about the benefits of Kangaroo Mother Care for hospitalised infants, especially within the NICU. Despite this knowledge, and the policies and protocols that support its implementation, $\mathrm{KMC}$ is known to be inconsistently applied within hospitals of affluent countries, such as New Zealand. The primary aim of this study was to work in collaboration with NICU staff towards an improvement in the quality of their Kangaroo Mother Care programme. A PAR approach was adopted because of its knowledge-sharing and emancipatory principles, exploring multiple participant perspectives, with a focus on participation and action-based change.

A typical PAR-project design was initally planned for three iterative cycles of participatory research, beginning with an exploratory phase and followed by implementation and evaluation phases. What eventuated in this case was a prolonged first exploratory cycle. This was largely due to diminishing active participation by the PAR participants and prolonged processes involving ethics applications and slow 
project development. At some time around the 2-year mark of fieldwork, it became necessary to decide whether PAR epistemology was being upheld for this study. This included assessing whether participation levels were satisfactory enough to remain aligned with PAR principles and, if so, what further methods of inquiry could satisfy PAR's requirements for ongoing participation from the NICU community.

PAR methodology proved to be adaptable and responsive to the complicated unfolding of the KMC project, providing scope for the adoption of further methods to address two major issues that arose in the study. Firstly, parental 'voice' in the primary research was represented by just one woman, which was problematic in that there was a risk of marginalising the lived experience of people in need of NICU services. In response to low parental participation, I undertook a review of local and global literature that foregrounded the persepectives of Māori women and whānau, as well as non-Māori with lived NICU experience from around the world. In support of increased perspective from the context of Aotearoa New Zealand, my own NICUstory was added. Secondly, in response to the finding of marked ambiguity between what was reported by the participants about KMC, and what was found in the research phases (know-do gap), I decided to conduct a discourse analysis for inquiry into underlying power/knowledge effects on KMC. Through feminist and Foucauldian lenses, a second research aim then sought to discover the predominant discourses and power relations influencing the implementation of KMC.

\subsection{KEY FINDINGS}

\subsubsection{Phases 1 to 5}

The most visible aspects of KMC were explored in phase one through five, where analysis revealed findings of KMC absence and ambiguity. Documentation of KMC was not comprehensive enough to reflect the policy guideline, with babies reported to receive $\mathrm{KMC}$ on just $44 \%$ of the days they were eligible to receive it. Whilst it was not possible to assess whether this was a true reflection of practice (rather than under-reporting, as suggested in feedback from the PAR group), the absence of documented $\mathrm{KMC}$ represents an alarming number of missed opportunities for $\mathrm{KMC}$, requiring urgent investigation. The overall finding was that $\mathrm{KMC}$ data from this NICU was not captured or measured and there was an absence of a measureable KMC quality improvement programme. 
In phase two, staff interviews found divergence and ambiguity of both individual and collective perspectives regarding Kangaroo Mother Care. Whilst KMC was embedded into both institutional policy and the awareness of the staff, its translation into practice was reportedly inconsistent, poorly prioritised and lacked educational support. In addition, there was a marked lack of clarity about where the responsibility lies for KMC implementation and quality improvement.

An observational study of Kangaroo Mother Care for babies in the predischarge phase of hospitalisation found infants had almost zero experience of KMC, which was inconsistent with the recommendations written into NICU policy. Despite the self-reported lack of resources for KMC described by some staff participants in phase two, neither environmental resources (such as comfortable chairs and screens), nor human resources (staff and parents) were limiting for KMC. Despite the indication for KMC with this group, and the infrastructure and personnel to support it, KMC was neither seen nor heard of over the course of the two-week observation period.

The fourth-phase narrative inquiry conducted with one recently discharged NICUparent detailed her experience of KMC during her prolonged hospital stay. The parent participant was introduced to KMC by a visiting midwife, not NICU staff, and regarded $\mathrm{KMC}$ as a necessity for bonding and the wellbeing of her and her daughters. Whilst most KMC experiences were positive for her, the parent somewhat reluctantly shared some of the complex thoughts and emotions that arose at times during KMC, termed by her as 'unwanted thoughts'. Based on her own experience, the parent advocated for much closer support for the mental health of caregiving parents on the unit, acknowledging the difficulty of her experience as 'mother of a hospitalised baby' and the challenge it posed to her own mental health. The mother found the education of KMC theory and practice to be inadequate. The estimated age of her babies at the time when this mother stopped providing KMC was about 34 weeks gestational age. The lack of KMC at this stage of development represents a gap in the care of her infants when compared with best practice evidence and policy, something of which she was unaware. This finding was reinforced by phase three, where other babies of similar gestational age were also not receiving KMC. Once again, this points to an institutional (cultural) characteristic of care, rather than an individually informed decision. 
The fifth and final phase was an interview with the longest standing PAR member of the group, a key KMC and developmental care stakeholder within the NICU. The resulting narrative revealed her concern about the 'perfect storm' that arose and contributed to the trauma experienced by babies in this NICU. In particular, parentinfant separation, prolific social ills and the negative lived experiences of the staff have led to a deterioration in developmental care values and implementation over the last decade. Societal and systemic factors such as increasing psychosocial complexity in the general population, chronic understaffing and insufficient funding at local and national levels, were all reported by her as negatively influencing the quality of care.

\subsubsection{Parental voices}

The second area from which key findings emerged was from local and global literature that reported womens', parents' and whānau/family perspectives about their lived experiences within the NICU, both specific and/or relevant to, KMC. Whilst the importance of sociocultural context was noted for the relevance of findings to different environments, there were common threads that emerged across womens' and parental voices that were important to represent. Women and families talked about the importance of their holistic needs - Kangaroo Mother Care was widely reported as important for bonding and feeling like an 'actual' parent. They described how important nurses were for facilitating this process and how quickly nursing staff could obstruct their bonding process . Indigenous women from New Zealand described how crucial it was to their wellbeing to include whānau support into the NICU routines without restriction. Other women and families described avenues for non-family support, as making a positive difference to their stays. People initially perceived the NICU as chaotic and frightening. People with lived experience of the NICU who identified as outside of the normative culture of the NICU, such as Māori or immigrants, were reported as experiencing extra distress. Parental recommendations for increased support generally involved psychosocial, cultural and/or spirtual factors, facilitated through nurses within a paradigm that acknowledged a more holistic picture of nurturing their baby.

\subsubsection{Discourse findings}

The third group of findings from this work relate to the influences of NICU discourses on KMC viewed through the lenses of feminist and Foucauldian theories 
about power/knowledge. Five main discourses, those of biomedical technocracy, health system discourse, humanism, nursing authority and the discourse of nurture were described. Biomedical technocratic discourse was found to have created a nearsingular authoritative knowledge that positioned medical professionals at the top of a hierarchical structure, with status to decide what care was enacted. Ongoing siloing and prioritisation of biomedical education and practice that are not as current or evidence-based as they could be. Discursive themes of safety/risk and the technological imperative, nurses as gatekeepers of infant care, and babies as patients not persons had the combined effect of marginalising parental knowledge and importance as the primary caregivers of their infants. The multiple discursive strategies in play combined to cause a displacement of the developmental care framework that was required to support an effective KMC programme. The findings from the discourse analysis support my assertion that the values and tenets of the normative biomedical technocracy were largely in opposition to those of KMC and the systems required to support it. The NICU healthcare 'system' was heavily focused on technology and medical interventionism and the institutional and practitioner values around which care was centred, rather than the holistic needs of the infant-parent dyad.

\subsubsection{Methodological findings}

The fourth and last group of findings relate to PAR methodology as a research approach in the complex social community setting of the NICU. PAR methodology afforded scope and flexibility for exploring the intricacies of the KMC programme in this NICU, with the possibility for responding to emerging findings in real-time. Embodying the principles of PAR made allowance for multiple pluralities and nonlinear research progress that ultimately moved too slowly for the timeframe of my $\mathrm{PhD}$ candidature. With high reflexivity, new methods and analyses were used within the PAR framework to respond to dwindling participation, making full use of the data made available within the first exploratory cycle. In addition, despite high complexity with respect to participant engagement in this case, paying close attention to the levels, positions and symmetry of participation was a reflective act that ultimately enabled the study to continue in congruence with the PAR approach (Jacobs, 2010; Penrod et al., 2016). 


\subsubsection{One NICU's KMC story}

In summary of the key findings, the essence of the KMC-story for this NICU is represented by an integration of the key findings from this study. The construction of this story is one version of 'truth', an interpretation from the perspectives of multiple community members. I attempt to avoid dualisms. I hold space for multiplicity through a critical feminist lens, remaining centred on participation, knowledgesharing and consciousness raising. There is no one 'voice' more or less important than another and I acknowledge the equal value of all perspectives. That said, the inherent social justice lens through which I view this research also behoves high reflexivity around the influences of discourse and power/knowledge. Particularly, who is empowered to speak, through what lens they do so, and what power is privileged with their knowledge. There is clear intention to foreground potentially marginalised voices within this space, those who speak quietly outside of the predominating paradigm of biomedicine.

Kangaroo Mother Care is suboptimally embedded into the culture and practices of this NICU, as evidenced by the findings of this PAR study. There is KMC absence and ambiguity, lack of available partnership; and missed KMC opportunities. However there is also KMC appreciation and the genuine willingness to improve it. Whilst promising glimmers are present in the form of positive perceptions about KMC, sustained and consistent practice is stopped short by institutional culture that is poorly constructed to support the practice. It is largely the systemic hypervaluation of biomedical theory and practice (to the exclusion of humanistic psychosocial principles) that discursively positions KMC at the bottom of a long list of medical priorities, consistently undermining the practice. It appears that the lived experience of staff within the unit is highly 'flavoured' by biomedicine, the technocracy of which infuses the whole of the NICU operation. General societal and public discourse, in addition, has been under the effects of internalised biomedical principles - 'Doctor knows best'. In combination with significant power relations within the NICU, discourse positions physicians and other medical practitioners in the position of authoritative expert and reduces parental 'voice' with respect to their infants' care.

A 'perfect storm' has increasingly arisen to marginalise the developmental care framework on this unit, consisting of increasing social complexity of incoming 
families, a healthcare community under duress due to the growing burden of noncommunicable disease and increased medical complexity of a small proportion of babies. Without a functional family-centred framework to default to, KMC is like a rudder-less boat on the ocean, subject to all manner of organisational and systemic pressures that continually compromise its sustained functioning. Lack of neurodevelopmental education, research and interdisciplinary cohesiveness further sideline the intervention, resulting in parents and staff not fully appreciating the extent to which KMC has been under-utilised. Ongoing tolerance of opinion-based care, as opposed to the operationalisation of evidence-based $\mathrm{KMC}$, remains a pillar of biomedical hegemony in this context. The result is maintenance of the status quo: physician/nurse-led, task-based care that operates outside of meaningful individualised patient-centred care. Worse, it is out of touch with the latest evidence for trauma-informed, developmentally appropriate, partnership models of support.

The perfect cultural storm provides a milieu that applies a broad-stroke approach to 'normal parents' of non-acute babies who are hospitalised on the unit for the purposes of feeding and growing. This group represent more than $70 \%$ of infants who are not in need of intensive medical care. Authoritative knowledge, applied (and often necessary) for the acute phase of hospitalisation, 'creeps' into the realms of both mother-baby craft and parenting, the phenomenon of which is enabled by societal discourse, parents and whānau/family themselves. One result is institutionalised parents who are reticent to take over increasing responsibility for their babies care, a phenomenon known by staff as 'NICU-itis'. Adaptation of the existing paradigm requires awareness, education and resource in support of nonnormative humanistic principles that uphold the psychosocial frameworks required for embedding KMC.

The exploration of KMC through a critical lens was firstly and most indelibly marked by absence. Absence within the case note documentation; reported absence of parents; absences of physician buy-in and active collaboration with KMC; absence of KMC discourse and practice for babies prior to discharge; absence of KMC for a parent and her babies' after after 34 weeks gestational age; absence of organisational support to enable $\mathrm{KMC}$; and absence of supportive integrative counter-discourse. The finding of absence of KMC was inter-related with ambiguity and dissonance in relation to the intervention. What was clear, was consensus that 
doctors had no professional responsibility for the education or implementation of KMC, short of being consulted for their opinion on safety to implement KMC with 'borderline' babies. As such, they remained guardians of safety, including the 'potential for risk' of $\mathrm{KMC}$, in itself creating cultural ambiguity about the safety (and therefore value) of KMC on the unit.

Partnership, in its many forms, is a mainstay of a functioning KMC programme and evidently a barrier within this unit to a more integrative care model. It is possible that the mutidisciplinary team meetings that are currently held have become a 'vehicle' for the dispersing of biomedical knowledge/discourse, exclusive of broader considerations including the KMC intervention. The technocratic model has a tendency toward dualistic and binary perspectives (either/or), a discursive tool by which 'other' (non-biomedical) considerations are positioned as non-normative. Care paradigms that support true partnership, shared decision-making and informed consent have within their value-system, a pluralistic view (both/and); that is, the capacity to hold multiple perspectives, a cultural facet of this community that is perhaps still in its infancy. 'Lip service' paid to parental-partnership, with even less inclusion of extended family members (such as grandparents), were self-reported by staff. In addition, meaningful relationship between various disciplines and tiers of responsibility may still be impeded by established lines of hierarchical status and professional-centrism. The cumulative effects of lack of significant partnership, with insufficient district health board and Ministry support, are likely to be involved in the enduring status quo model, and counter-productive to the quality improvement of a non-medical intervention such as KMC.

Whilst a shift in the paradigm of the culture of care within this NICU is required for the maximum upscaling of KMC, there were visible daily routines that could be used as vehicles for its increased use. Enhanced KMC implementation could conceiveably occur without any immediate modification to the systems issues identified by this research. The 'missed opportunities' for KMC could be attended to by little more than increased awareness by the staff and informal, bedside mentoring sessions engaging parents - a bottom-up approach. In addition, existing staff educational days, particularly those attended by various medical, nursing and allied health professionals, provide a means by which to integrate 'non-medical' interventions into the syllabus. Foregrounding the status of KMC as an evidence-based method for 
improved quality of care, enhanced short and long-term health outcomes and an urgent humanitarian matter is an important whole-group activity. The NICU staff could use the existing hierarchical model as it stands, to provide a united front of information for dissemination through 'top-down' means. Missed opportunities for KMC abounded. There were times when the baby was already going to be undressed for bathing and/or weighing; times when they were being cradled using a conventional cuddle for feeding; and times when people were soothing babies while they were receiving painful procedures such as heel-sticks. People cannot implement what they don't know, and it was a clear finding from this study that the evidencebased knowledge and education of KMC was somewhat lacking in this community. Juxtaposed with a critical approach for identifying the gaps of $\mathrm{KMC}$ on this unit, the findings of endemic KMC appreciation and a willingness to improve the current situation were both highly visible. Individuals really cared about KMC. They were obviously heavily influenced by a system that was restrictive of developmental care and KMC, however they did the best they could with the knowledge and resources which they had. I concur with lessons learned from developing countries who are heavily engaged with KMC quality improvement, that "The service provision of the relevant healthcare focus areas (KMC) needs to be institutionalised and not individually driven, in order to reduce fluctuation in service delivery..." (Feucht et al., 2016, p. 51). The benefits of KMC, present in policy, were certainly embedded within the staff and parental psyche. In addition, champions existed who were highly reflexive and aware of the harm that was occuring as a result of the lack of an efficient developmental care model by which to structure KMC. There was a huge untapped resource within these champions as change agents, not least their capacity to work immersed in the complexity of this environment with the accompanying tension of the 'ideal' versus the 'actual'. Change agents for KMC maintained a willingness to improve the quality of their programme, readiness to innovate and find 'another way' to do so, and represented the grass roots leaders of a community who were aware of their need to do better for their vulnerable patients. The integration of individual efforts with necessary systemic change exist as promising keys for activating KMC improvement within this NICU.

At the limits of the findings of this research and to end this story of KMC for now, I unequivocally advocate for a shift in the systemic paradigm, in support of a more 
integrated holistic biomedical approach to hospitalised infant care. To this end, the application of KMC may be viewed as both a cause and effect of more humanised medical principles, further recommendations for which will be outlined in 7.5.

\subsection{CONTRIBUTION TO KMC KNOWLEDGE}

This is the first doctoral study to explore quality improvement of KMC within the high-income NICU setting, using a PAR approach. The findings contribute to raising awareness about how Kangaroo Mother Care, an intervention positioned outside of the predominating biomedical discourse, may be under-utilised due to the unexamined power relations of a medically-privileged knowledge system. In addition, the specific findings from the first five phases of the study have contextual implications for this NICU's KMC programme, that may be transformative to this group's practice. The findings generated in this study, therefore, have specific and broad significance for KMC in both the NICU involved and for the wider national and international NICU communities.

Whilst multiple recent systematic reviews have reported health system barriers to KMC in various settings, there has been no literature reporting deeper analysis of the effects of the organisational paradigm on KMC in high-income settings. In their systematic review of 86 qualitative studies, Chan and colleagues (2017) recommended analysis of health system perspectives of $\mathrm{KMC}$ to advance its uptake in various settings. They cite worldwide barriers to KMC within health facilities such as "management reluctance to allocate space for SSC (skin-to-skin contact)" and "high leadership turnover" (Chan et al., 2017, p. 5). Certainly, a number of the health system and practitioner-level barriers to KMC identified as themes within the worldwide literature were also findings from this study, including: lack of practitioner and institutional buy-in (Chan et al., 2017; Seidman et al., 2015); insufficient social support and empowerment (Chan et al., 2017; Seidman et al., 2015); real or perceived time pressure (Chan et al., 2017; Seidman et al., 2015); medical concerns about the infant (Chan et al., 2017; Seidman et al., 2015); insufficient access to training and resources (Chan et al., 2017; Penn, 2015; Seidman et al., 2015); and sociocultural norms of the organisation and/or individual (Chan et al., 2017; Penn, 2015). While these barriers are no-doubt present and important, this research, through the adoption of a discourse analysis, has explored the deeper and previously unexamined paradigmatic influences on many of these systemic barriers. 
Similarly, a recent literature review of KMC barriers in the United States identified that staff attitude, knowledge and "the demands of a hospital" (Penn, 2015, p. 25), all presented obstacles to the sustained functioning of KMC in the hospital setting. Another quality improvement study of couplet care for healthy full term-infants described the successful transition of a nursery-based care model to mother-baby couplet care, without a more in-depth inquiry into characteristics of NICU culture. It was the author's conclusion that "implementing any new model of nursing care is a tremendous culture change for both nursing staff and physicians" (Brockman, 2015, p. 502), with the sustained change on their unit representing a 'culture shift'. Whilst one published paper describes the protocol of a PAR study of family-centred care within an English NICU (including Kangaroo Mother Care), the findings of that study have yet to be described (Skene et al., 2015). To date, therefore, published literature lacks findings, implications and recommendations that relate to inquiry into the paradigmatic characteristics of the culture of care affecting KMC.

It is my assertion, therefore, that a gap in current $\mathrm{KMC}$ literature exists relating to the paradigmatic attributes that affect its implementation, including which methods researchers may use to explore this aspect of the NICU institution. The research I undertook in partnership with members of one NICU has confirmed healthcaresystems issues affecting KMC similar to the established literature. As well, it has provided further evidence about the power relations that contribute to the enduring lack of a sustainable KMC programme. The discursive findings from this study provided substantive evidence that the biomedical, technocratic influences on NICU culture are likely to be highly influential on the humanistic care method of KMC. In addition, I argue that without the identification and awareness of the KMC-related discourse within each setting, efforts to create sustained system change are likely to be hampered. Inquiry into the discourse which reflects the paradigmatic 'operating system' of the NICU, including its influence on KMC practice, is therefore an important aspect of quality improvement.

\subsection{PAR CONTRIBUTIONS AND REFLECTIONS}

Participatory action research holds the capacity for transformational change through collaboration with the community for which the research involves. This research made two major contributions to the field of PAR methodology. Firstly, it answered the call for scientifc method to enhance understanding of patient/family-centred care 
through the participation of consumer and staff with lived experience of the NICU environment. It did this through reinstating patient and staff stories, the “epistemological legitimacy” (Bradby et al., 2009, p. 331) of which has brought narrative to the NICU environment. Secondly, PAR provided scope for the use of a Foucauldian-informed discourse analysis, a method by which the significant power relations and predominating cultural paradigm was explored.

Participatory action research was seen as a suitable choice for a quality improvement project. The combined characteristics of KMC , the vulnerable population, the high acuity environment and the nature of healthcare organisations, all create a complex social system that requires the buy-in of its members in order to create sustainable change. If required, the wide scope of PAR as an 'approach', as opposed to a rigid set of methods, provides an avenue to explore action-based change within the dynamism and intensity of the NICU.

Participatory action research principles require ongoing mutual agreement for actionbased change, at every step of the process. As PAR members left the group for multiple reasons, dwindling to a single remaining key NICU stakeholder, a necessarily modified approach to PAR was forged. Although a 'classical PAR' project was not borne out in this case, my intention to remain committed to PAR principles has resulted in findings that are as trustworthy as participatory in nature. Ultimately, a collaborative effort was achieved, when despite the challenges to maintaining positive forward momentum, the loop on the first full exploratory PARled cycle was closed. Findings were passed back to the group which could then be used to set the stage for subsequent cycles of KMC quality improvement using PAR, if chosen by the community.

The use of a Foucauldian-informed lens and discourse analysis for this study also provides a significant contribution to the field of PAR knowledge. Change theory literature from complex systems such as the NICU commonly recommends organisational culture as a potential enabler/barrier of practice, yet provides few tools for its examination. To this end, inquiry into power relations through discourse analysis with feminist and Foucauldian lenses, provided one such tool. It enabled a more holistic representation of KMC than that provided by the first phase findings, hence maximising the richness of data that was collected over the course of the study 
(Altman et al., 2014). Whilst discourse analysis remains a poorly described methodological choice within the literature for this sort of implementation research, it was found to align particularly well with the epistemological stance of PAR. It was therefore a well-rationalised decision to adopt a discourse analysis late in the course of the study for a more detailed examination of the data relating to the unit's KMC programme.

As significantly, the major contribution that the applied feminist lens made to the PAR approach is highlighted here. At its heart, this research strived to uphold values esteemed by the PAR principles, many of which are held at the centre of feminist science. Most notably, those of inclusivity, power-sharing and the representation of multiple truths, the necessity to accept plurality and giving equal weight to all participating voices. I became aware that the presence of tension within myself was often due to difficulty in acknowledging the multiple truths that existed amongst NICU-community members toward KMC. It was this tension that highlighted the requirement for a deeper self-inquiry into my personal feminist worldview and presence it more consciously in this work.

I also personally experienced the paradox written about within feminist literature; that of the dissonance created by the use of a critical lens for action-based change, whilst attempting to collaborate constructively with research participants. Scholars have theorised that if beliefs and practices are to change, then some amount of 'destruction' is inherent in emancipatory-based research (Jenkins, 2015). I can only surmise that it is not the intention of most researchers to inflict damage on a group or system (it certainly wasn't mine), however, efforts may be perceived in that way by participants. Where the identification of a need to evolve practice is highly visible, researchers should be aware that there will likely be at least some resistance to change within the group. I remain hopeful that the addition of feminist principles to this study will support participants to view my interpretation as "one of several possible versions" (Jenkins, 2015, p. 16). It should be considered, however, that the many engrained protective structures that endure to serve the institutional status quo, both personally and collectively, reinforce the closed door policy on generating constructive engagement. 
One consistent criticism of PAR methodology is that the results are highly contextual and limited to the population of participation and are therefore not generalisable. I would argue that the 'bones' of the project design are highly transferable. Creating a PAR project within the NICU environment could potentially be modelled on the methodological approach and design of this study.

\subsection{LIMITATIONS}

The potential for limitations to the findings of this study relating to participation are noted. The voice of just one woman from the participating NICU, whilst extremely valuable as a single narrative, would have been strengthened by more input from the NICU community involved. In response, the addition of my own narrative and findings from women and family outside of this setting go some way to avoiding the marginalisation of perspectives from people with lived experience as consumers in the NICU, whilst also demonstrating the scope of PAR to address real-time shortfalls as they arise. Another important absence was the lack of participation by Māori women and their whānau. I acknowledge my own and other healthcare researcher's commitments to the improved quality of Māori health outcomes and improved cultural competency in Aotearoa New Zealand. In doing so, I foreground that the absence of Māori voice in this research was likely due in some part to institutional racism (Theunissen, 2011). In future, I would adopt a more explicit bicultural framework in the preliminary planning phases of a PAR project, thereby holding space for indigenous perspectives and actively seeking Māori participation.

Another methodological challenge was the restriction of this project to just one exploratory cycle of PAR, rather than the expected three-cycle process. Whilst the contributing factors to this have been described earlier in the thesis, it was the stilted ethical application processes that I would attempt to change for future projects. Given the relative novelty of PAR in healthcare research, I would include discussions about PAR with ethical review boards within the preliminary planning phase, helping to ensure ethical applications unfolded in a more timely and consistent manner. Whilst the presencing of PAR methodology within the NICU of this study was undoubtedly 'novel', I argue that the approach was not mutually exclusive to traditional scientific approaches in this high-acuity healthcare space. Given that there will likely be an increasing demand for participatory processes 
within health research, there is an undoubted call to action for research ethics committees to update knowledge and infrastructure to accomodate PAR processes. That said, despite ethical review board factors, the project stands alone as a full and complete first PAR cycle of KMC exploration.

Lastly, where researchers do not fully describe the processes of their PAR studies, some scholars contend that findings are lowly transferable to other studies and contexts (Carcary, 2009; Langlois et al., 2014). The detailed audit trail process and the integrity of the application of PAR principles in the case of this research were both intended to avoid the potential for this limitation.

\subsection{RECOMMENDATIONS BASED ON THIS STUDY}

This study has advanced knowledge into the field of maternal-infant health in the area of Kangaroo Mother Care for preterm infants within the NICU environment. The principles of participatory action research methodology, in conjunction with Foucauldian and feminist lenses, were applied to the exploration of KMC using multiple methods. The methodological contributions from this one context can be applied to other populations, both within the context of Aotearoa New Zealand and internationally. Specific recommendations in support of their KMC programme will be made next for this NICU, followed by broad recommendations for healthcare organisations caring for infants, mothers and families who plan to upscale their KMC efforts.

\subsubsection{NICU-specific recommendations}

These recommendations are based on the findings from this study and the integration of a consensus-based KMC policy statement for the widespread use of KMC for premature and low birthweight babies (American Academy of Pediatrics et al., 2016). KMC scholars have recently been working toward a more standardised approach to KMC development, represented in a measurement framework informed by the World Health Organisation and based on KMC Service readiness (Guenther et al., 2017). Guenther and colleagues (2017) describe areas of focus that include leadership and governance, financing, health information and delivery, the health workforce, essential resourcing, and advocacy for community ownership and partnership. Increasingly, it is the building of healthcare capacity through development of policy and infrastructure, combined with widescale 
acknowledgement of the emotional implications of hospitalisation, that are supporting the construction of "trauma-informed systems" (Ash \& Williams, 2016, p. 317). The following recommendations are made to the NICU involved with this study, in support of their transition to a more cohesive trauma-informed capacity through quality improvement of their KMC programme and developmental care framework (table 13). Recommendations in table 13 were compiled in collaboration with co-participants including myself, NICU staff and one NICU parent. 
Table 13: NICU-Specific Recommendations for Quality Improvement of KMC

\begin{tabular}{|c|c|}
\hline $\begin{array}{l}\text { LEVEL OF } \\
\text { OPERATION }\end{array}$ & RECOMMENDATION \\
\hline $\begin{array}{l}\text { ORGANISATION } \\
\text { Leadership and } \\
\text { governance } \\
\text { Health financing } \\
\text { Health information }\end{array}$ & 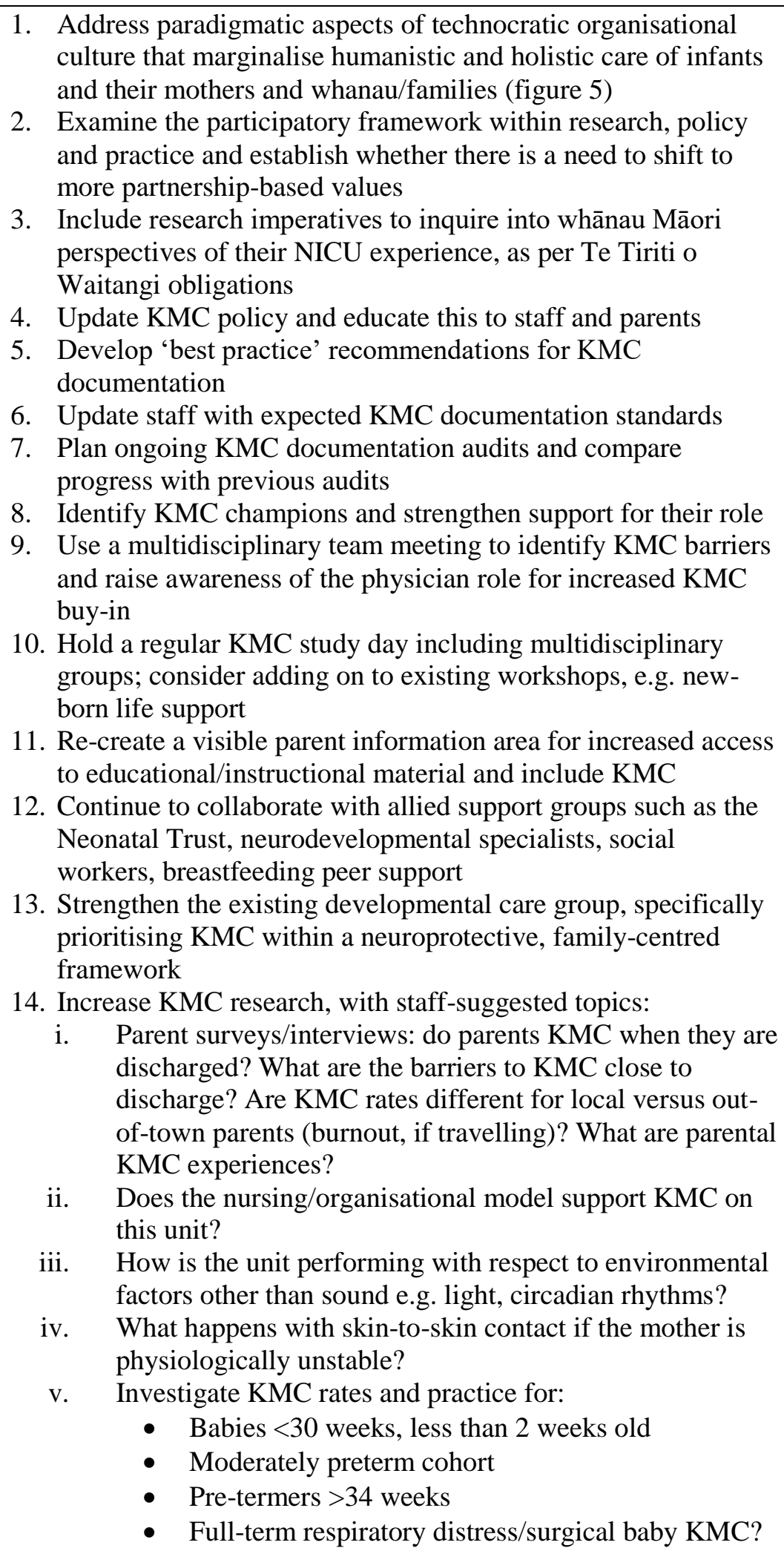 \\
\hline
\end{tabular}




\begin{tabular}{|c|c|}
\hline $\begin{array}{c}\text { LEVEL OF } \\
\text { OPERATION }\end{array}$ & RECOMMENDATION \\
\hline $\begin{array}{l}\text { STAFF AND } \\
\text { PARENT } \\
\text { Health workforce } \\
\text { Community } \\
\text { ownership and } \\
\text { partnership }\end{array}$ & $\begin{array}{l}\text { 1. Create a 'competency checklist' of KMC skills for increasing } \\
\text { staff and parental confidence } \\
\text { 2. Identify existing care practices where KMC may be increased } \\
\text { e.g. bathing, weighing, undressing, feeding, during medical } \\
\text { procedures } \\
\text { 3. Increase formal KMC education of parents including dads, } \\
\text { wider whānau, and grandparents. Consider combining with } \\
\text { education on cellphone behaviour and breastfeeding support } \\
\text { 4. Consider moving to the original four-part KMC model using } \\
\text { continuous KMC rather than intermittent SSC } \\
\text { 5. Educate parents on leading KMC with older/stable/clothed/less } \\
\text { preterm babies, once they are in cots } \\
\text { 6. Increase staff education with KMC modules on standing } \\
\text { transfer and reading neurodevelopmental cues } \\
\text { 7. Establish a KMC peer support group comprised of ex-NICU } \\
\text { parents, nurses, managers and physicians from Māori and non- } \\
\text { Māori groups }\end{array}$ \\
\hline $\begin{array}{l}\text { ENVIRONMENT } \\
\text { AND } \\
\text { RESOURCES } \\
\text { Essential supplies }\end{array}$ & $\begin{array}{l}\text { 1. Review suitability of space for KMC, especially if a KMC } \\
\text { upscale is planned } \\
\text { 2. Monitor KMC equipment (chairs, screens) for shortfall } \\
\text { 3. Continue to educate staff on the importance of the sensory } \\
\text { environment for the baby, especially with respect to noise, } \\
\text { lighting and cellphone use } \\
\text { 4. Innovate a safe and reliable KMC wrap }\end{array}$ \\
\hline
\end{tabular}

In addition, I have suggested a paradigmatic reframe that I have called 'from NICU to NIPU', where NIPU is an acronym for Neonatal Intensive Parenting Unit, a concept recently introduced by neonatal researchers and scholars (Hall et al., 2017).

A paradigm shift that acknowledges and applies humanistic and holistic principles, raises awareness about the lack of sustainability of systems that function to usurp basic biological human needs for the sake of cost, tradition and the status quo. In support of this paradigm shift, I have suggested counter-narratives away from the current predominating NICU discourse found in this setting, assisting those who adopt them to shift their thoughts and actions toward improved integration of KMC and humanistic frameworks (figure 5). 
Figure 5: From NICU to NIPU - a Paradigmatic Reframe

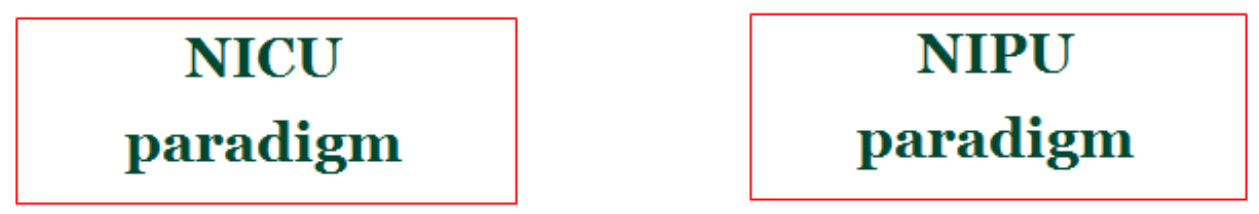

PARADIGM SHIFT» »»

\begin{tabular}{|l|l|l|}
\hline \multicolumn{1}{|c|}{ CHARACTERISTIC } & \multicolumn{1}{c|}{$\begin{array}{c}\text { CURRENT } \\
\text { NARRATIVE }\end{array}$} & \multicolumn{1}{c|}{$\begin{array}{c}\text { COUNTER- } \\
\text { NARRATIVE }\end{array}$} \\
\hline Hegemonic model & Technocratic/Biomedical & Humanism/Holism \\
\hline Centre of care & Practitioner-institution & Infant-caregiver \\
\hline Dominant discourse(s) & $\begin{array}{l}\text { Fear-based, risk averse } \\
\text { Medical technocracy } \\
\text { Technological imperative }\end{array}$ & $\begin{array}{l}\text { Love, nurture, wellbeing } \\
\text { Trauma-informed care } \\
\text { Family-centredness and } \\
\text { connection }\end{array}$ \\
\hline Orientations & $\begin{array}{l}\text { Overstatement of risk } \\
\text { Authoritative knowledge } \\
\text { (physician, medical) } \\
\text { Disease-focused } \\
\text { Short-term focus } \\
\text { Nurse-led, nursery based infant } \\
\text { care, separation is expected }\end{array}$ & $\begin{array}{l}\text { Shared knowledge, multiple truths } \\
\text { Biopsychosocial optimisation } \\
\text { Mother-infant dyad preferentially } \\
\text { supported } \\
\text { Parent-led, attachment-based, } \\
\text { unity is upheld } \\
\text { Life-long health }\end{array}$ \\
\hline Values & $\begin{array}{l}\text { Interventionism } \\
\text { Opinion-based care based on } \\
\text { medical tradition } \\
\text { Expert-driven, hierarchical } \\
\text { Psychosocial elements of care } \\
\text { undervalued }\end{array}$ & $\begin{array}{l}\text { Parent is the most valuable } \\
\text { caregiver } \\
\text { Individualised care } \\
\text { Peer support } \\
\text { Socio-emotional wellbeing }\end{array}$ \\
\hline
\end{tabular}

\subsubsection{Broad NICU recommendations}

Based on this work, the following broad recommendations are made to researchers and community members interested in using a participatory approach within their NICU for quality improvement of KMC:

i. Participatory Action Research methodology is a worthwhile approach to producing new knowledge, associated with a shift to patient-centred models and humanistic care within the complex environment of a NICU. Its use is recommended to support a collaborative integration of grass-roots and topdown activity for the transformation toward equality and democratisation.

ii. Where ambiguity and/or dissonance is present (often visible as a 'know-do gap' and/or slow or obstructed research progress), the adoption of methods for examining organisational culture are pivotal to understanding paradigmatic influences on knowledge translation. 
iii. Consider the overarching cultural paradigm affecting KMC within each NICU and whether development of suggested counter-narratives would be beneficial for reframing the predominating ones (figure 5).

iv. Discourse analysis is a recommended adjunctive method for the examination of power/knowledge constructs within the institutional NICU setting, where the paradigm remains largely biomedical and technocratic. PAR enables the use of a wide scope of methods for responding to emerging findings.

v. Feminist and Foucauldian principles are highly aligned with PAR and recommended as methodological lenses in the NICU environment because of the relationships between women, infants, families, healthcare and enduring patriarchal authority. These lenses are helpful for informing a critically constructive approach.

vi. Examination of participatory frameworks and pathways for quality improvement of KMC are essential to support change. Closing the quality improvement 'loop' for KMC programmes is required through increased accountability with the implementation process. Audit, professional development and iterative cycles of evaluation are required.

vii. Awareness of realistic and available timeframes will assist participants in deciding the size and scope of the project, given that PAR cyles of exploration, implementation (ideally, three) and evaluation are likely to take at least 18 months to enact.

viii. Researchers who plan to facilitate a PAR project within an organisation in which they don't work ('outsiders') are encouraged to consider a lengthy preliminary planning phase that involves relationship building, continual reassessment of buy-in and participation with the project. Ask, is a sustainable PAR-group forming?

ix. I recommend the use of Kangaroo Mother Care indicators for maternal-infant care within all settings, if not already applied. This should be introduced systemically at the level of the Ministry of Health (or equivalent). This recommendation is based on Kangaroo Mother Care being the one evidencebased constant of all developmentally-appropriate family centred frameworks.

x. Where ethical review boards are unfamiliar with the PAR approach, consider planning for lengthy ethical application processes. I would recommend 
approaching ethical review committees for some clarity on whether they have worked with PAR applications previously, and if not, providing committees with literature which they can use for educational purposes.

Contextual differences aside, a theoretical framework for PAR projects within the NICU space may be used to design a quality improvement study for a KMC programme, irrespective of where it is in the development or implementation stage. I recommend the following additions to the suggested PAR framework. Firstly, the facilitating researchers are themselves a source of data through the use of their own field notes, observations, and perspectives about the intangible paradigmatic aspects of organisational culture. This awareness affirms the 'voice' of the researcher as a co-participant in the study and potentiates the collaboration between 'researcher' and 'researched'. This act simultaneously encourages researcher reflexivity and increases accountability of their observations, due in part to the analysis of their observations as data. Secondly, addition of a method of inquiry that focuses on the political and sociocultural environment of the NICU is both warranted and necessary. Without examination of the power relations that affect the lived experience of the participants who are often unwittingly exposed to them, only part of the 'puzzle' is elucidated.

\subsection{CONCLUSION}

This research represents a very personal and scientific endeavour that was undertaken for humanitarian and social justice reasons. The vision was to give voice to those who cannot tell their own story, the vulnerable individuals who require hospitalisation under the suboptimal conditions most usually associated with early and/or problematic birth. Each individual, family and caregiver within the milieu of the NICU, has a story. The intersections of each story within the NICU needs to centre on the support of healthy development and the reduction of suffering for those enduring trauma in the postnatal and early infancy period. Synthesis of the established evidence-base for biological expectations of the maternal-infant dyad when babies are in infancy leads me to to state this unequivocal KMCrecommendation to all those involved with infant support in the NICU:

Kangaroo Mother Care, for the majority of babies, for the majority of the time, provides a science-based solution that is cost-effective, results in improved health outcomes and is a necessary humanitarian response to the trauma of hospitalisation. To this end, support by the Ministry of Health and NICU leadership is required to effect a paradigm shift toward a more humanistic, medically-integrated approach to 
care. Scientific methodology that raises the consciousness of individuals at the coalface of NICU-based maternal-infant care, is required for effective translation of theory into practice and is a matter of urgency for this highly vulnerable group.

Through this research, an original contribution of knowledge is made to the KMC programme of one NICU community in Aotearoa New Zealand. As well, there are contributions to the larger global NICU network for which evidence-based KMC is strongly advocated. The Foucauldian- and feminist-informed PAR approach used for this study found multiple societal, organisational, staff and parent/whānau/family factors affecting KMC in this environment. The first exploratory cycle of PAR laid a foundation for ongoing cycles of research within this unit, as well as provided a description for how the approach may be embodied for use in other contexts, particularly those of high-income countries.

Of particular note are the overarching paradigmatic influences of biomedical hegemony on KMC, exerted through the positioning of all NICU community members via technocratic discourse. The lack of funding/resource reported by staff of this community is incongruous with the evidence-base about the low-cost-lowtech trait of KMC that has shown to decrease healthcare costs in low- and middleincome countries through multiple means: reduced length of hospital stay, increased growth rate, decreased morbidities, and increased input from parents/whānau/families (Bergh et al., 2012a; Boundy et al., 2015; Evereklian \& Posmontier, 2017; Ruiz et al., 2016; Uwaezuoke, 2017). Whilst caution is advised in generalising findings such as cost-effectiveness into a new context, there is strong rationale for suggestion that cost-effectiveness of KMC, when compared with conventional care, is transferrable from low and middle-income settings to highincome units (Syed et al., 2012). I encourage the leaders of systemic change within this NICU to consider that insufficient funding discourse is used as an organisational tool to maintain the status quo of conventional care, and not grounded in the evidence-base of the cost-effectiveness of KMC. Findings from this study support the fact that resources are present to upscale $\mathrm{KMC}$ and it would be unethical to not do this, given the results of this inquiry.

There is an emerging inquiry into the politics of neoliberal medicine and its potential relationship to NICU overuse, with scholars recommending further research to uncover more about the phenomenon of NICU use for modern-day infants. To emphasise the emerging awareness of (often unacknowledged) sociopolitical 
ideology on medicine, one scholar describes the deterioration of their model of KMC in Columbia, at least partly due to its characteristic as a 'non-commodifiable' (and therefore non-profitable) approach to medicine (Abadia-Barrero, 2018, p. 23):

Given the raising costs of technology, health care inequality, and unacceptably high infant mortality rates around the world, one would think that KMC would have become hegemonic in the care of compromised neonates. This did not happen, however.

Whilst it remains a theoretical extrapolation to begin to respond to the ideological questions of these researchers, the findings from this study provide evidence of medicalisation of infant care that may represent one variance of NICU overuse, informed by the biomedical hegemony. Inquiry using discourse analysis was a powerful tool for interpretation of the discursive strategies and subject positioning inherent within the power relations of this NICU environment. It is a well reasoned recommendation to apply power analyses to other NICU communities where unexamined power relations are considered to be influential and a barrier to translation of KMC knowledge into practice.

This research supports and intensifies a call-to-action from the World Health Organisation (2015) and other maternal-infant health advocates, for protection of the postnatal experience of hospitalised infants through upscaling of KMC (Als et al., 2011; Altimier \& Phillips, 2016; Bergman, 2015; Chan et al., 2016; Conde-Agudelo \& Diaz-Rossello, 2016; Feldman et al., 2014; Feucht et al., 2016; Hubbard \& Gattman, 2017; Luong et al., 2016; Nyqvist, 2016). Evidence of the suboptimal functioning of $\mathrm{KMC}$ on this unit, suggestive of an overarching systemic failure to support the method, is unlikely to be an isolated case. Current knowledge supports the perspective that underutilisation of KMC is likely to be endemic in other units both in this country and in other high-income settings, which is certainly supported by this study. Based on science, we are now incontrovertibly informed that the wellbeing of some of the most vulnerable of our citizens - hospitalised infants depends on the holistic and loving care in their postnatal stage of development. Suboptimal care in this period affects babies over the course of their entire lifespan through multiple biological mechanisms, the mitigation of which is undeniably urgent. Integration of the most current neuroscientific principles of human infant development tells us firstly, what universal human infant needs in the postnatal period are, and secondly, how lack of awareness of those needs may lead to 
unintentional neurobiological neglect. Ultimately therefore, the essence of this KMC quality improvement project is one of advocacy for human rights and humanitarian principles within the NICU space. The application of a highly functioning Kangaroo Mother Care programme and the trauma-informed neuroprotective care it affords, must be considered a non-negotiable gold standard for the care of babies and their whānau/family.

Humanisation of the NICU environmental space, however, involves more than just the knowledge possessed by scientific scholars. Sustainable change in this case also requires a higher vision centred on humanitarian values, as well as knowledge of methodologies by which to strategically activate the desired transformation. National healthcare ministries and local NICU communities are required to commit to partnership that serves the needs of the humans who both use and contribute to the NICU social system. PAR methodology, underpinned by principles of raising critical consciousness through participatory activity with the group whom the transformation will affect, provides a vehicle for this. It is in this direction that modern neonatology is being asked to move, supported by the findings and conclusions of this research. I wholeheartedly invite future researchers and participants to engage with PAR research for the explicit purpose of KMC improvement. The PAR approach is adaptable and capable of enabling the crucial development and upscale of KMC for this exquisitely vulnerable population. Through its systematic application and, if needed, modification, the possibility of action-based change may be realised and sustained. Future research is urgently required in the area of organisational paradigmatic influences that block effective KMC upscaling. The addition of knowledge from the story that this thesis tells, pays participants' efforts forward into raising awareness of KMC upscale in the high-income NICU setting. The desired and optimal outcome of a highly functioning KMC programme is the improvement of short and long term health of hospitalised babies, their parents and the folk who support them.

When making humanisation of NICU healthcare the top and non-negotiable priority, we not only speak for the most vulnerable in our society, we also commit to building a healthier future population. In doing so we protect the present through the most sacred of medical commitments: first, do no harm. Kangaroo Mother Care is the glaring choice for humanistic care of NICU babies, their mothers and families. In 
CHAPTER SEVEN: DISCUSSION

support of functioning and sustained KMC implementation, there is no price too high to pay. Indeed, our humanity may just depend upon it. 


\section{APPENDICES}

\section{Appendix 1: Health and Disability Ethics Committee, Approval 2}

Health
and
Disability
Ethics
Committees

Health and Disability Ethics Committees Ministry of Health 133 Molesworth Street

PO Box 5013 Wellington 6011

08004 ETHICS hdecs@moh.govt.nz

21 February 2017

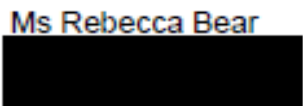

Dear Ms Bear

Re: HDEC ref:

\section{$17 /$ NTB/33}

Quality Improvement of Kangaroo Mother Care in one tertiary Neonatal Intensive Care Unit: A Participatory Action Research Project

Thank you for submitting your application for HDEC review on 21 February 2017. The Secretariat has assessed the information provided in your application and supporting documents against the Standard Operating Procedures.

This application has not been validated, as on the basis of the information you have submitted, it does not appear to be within the scope of HDEC review. This scope is described in section three of the Standard Operating Procedures for Health and Disability Ethics Committees.

Your application describes a quality assurance evaluation of kangaroo mother care in the District Health Board Neonatal Intensive Care Unit. The focus of the evaluation will be on the pre-discharge phase of hospitalisation. By conducting this project the outcomes of babies in the pre-discharge phase at $\square$ NICU will be improved.

If you consider that our decision not to validate this application is in error please contact us as soon as possible giving reasons for this.

This letter does not constitute ethical approval or endorsement for the activity described in your application, but may be used as evidence that HDEC review is not required for it.

Please don't hesitate to contact us for further information.

Yours sincerely,

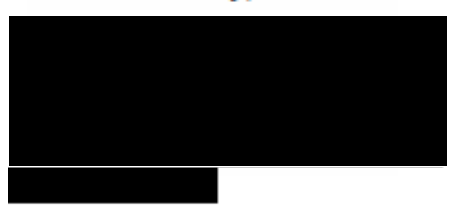

Advisor

Health and Disability Ethics Committees 


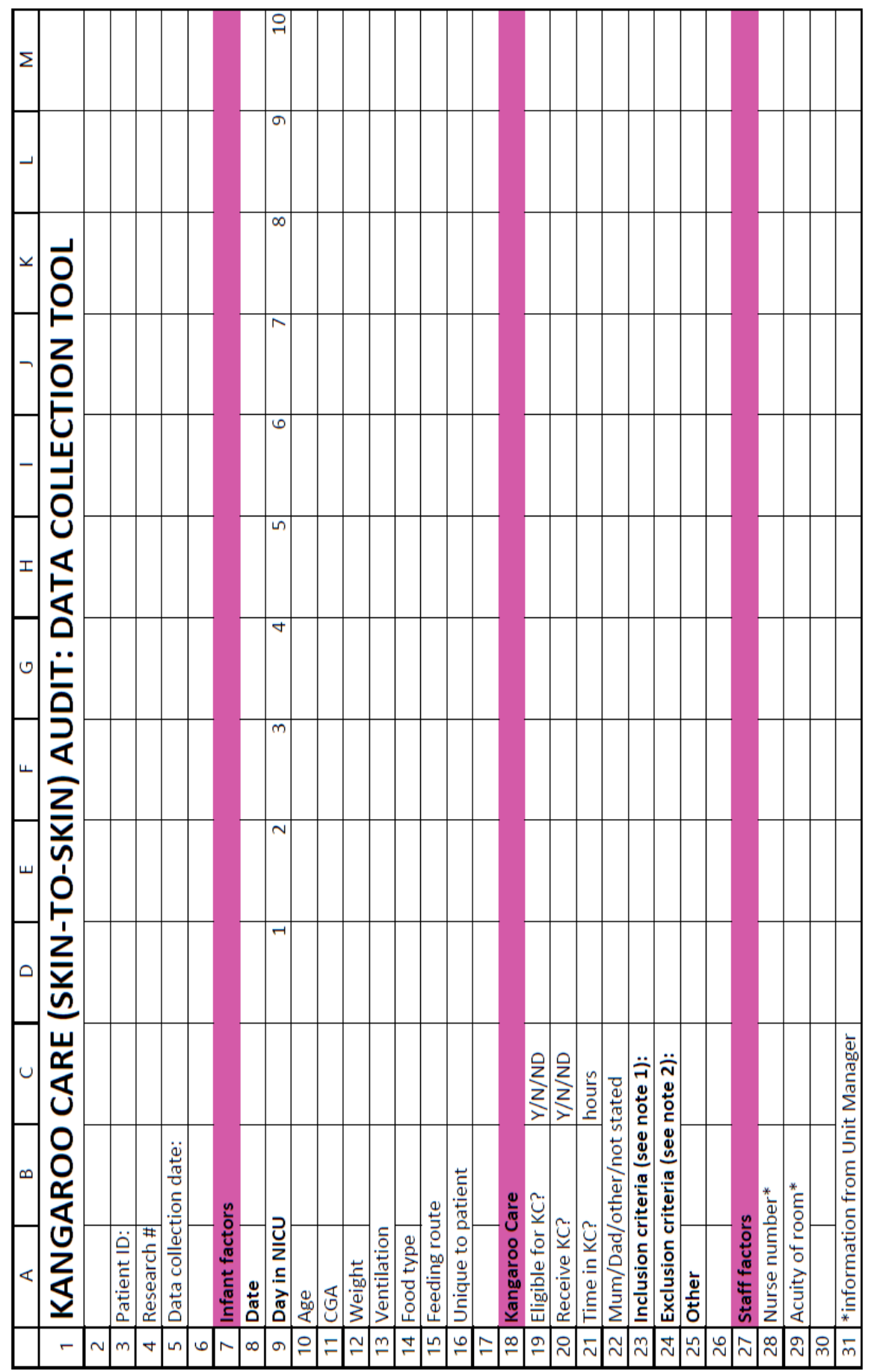




\section{Appendix 3: KMC Audit Approval}

Document Facilitator: Cllnical Audit Advisor Senior Document Owner: Quality and Risk Manager Document Number: Errorl Unknown document property name. Issue Date: 15 January 2013

Review Date: 15 January 2016

\section{Appendix 3 Clinical Audit Registration and Proposal Form}

\section{Instructions}

- Please use this form to help plan and register your project. Registration allows you to receive constructive feedback on your work and information on any resources avallable which may be of use to you.

- This form should be completed by all employees and submitted to your manager for approval of resources and then forwarded to the appropriate Committee/Group.

- All fields are mandatory with the exception of Section 2.5.

- If you require any assistance in completing this form please contact the Clinical Audit Advisor or your directorate Quality Manager.

\subsection{Audit Personnel}

1.1 Audit Facilitator Details

\begin{tabular}{|l|l|}
\hline Name: & \\
\hline Contact number: & Y \\
\hline \multicolumn{1}{|c|}{ employee } & \\
\hline If N please provide details & \\
\hline Job title \& discipline: & ACNM; Neonatal Intensive Care Unit \\
\hline Ward/unit/department: & Neonatal Intensive Care Unit. \\
\hline Service: & Women's Health Service \\
\hline
\end{tabular}

1.2 Audit Team Members? N (If N, skip to Section 2.0)

\begin{tabular}{|l|l|l|}
\hline Name(s): & Job tite(s) \& discipline(s): & Contact number: \\
\hline $\begin{array}{l}\text { Rebecca Bear (conducting } \\
\text { audit) }\end{array}$ & $\begin{array}{l}\text { PhD candidate } \\
\text { Graduate School of } \\
\text { Nursing, Midwifery and } \\
\text { Health, Victoria University } \\
\text { of Wellington }\end{array}$ & $049053900 ; 0212220900$ \\
\hline
\end{tabular}

\subsection{Audit Project Details}

\subsection{Rationale for Audit}

\section{Please $\checkmark$ all that apply}

Recent relevant guideline published: *due for update in September 2015

Clinical policy

Evidence of a quality problem e.g. incidents, complaints, high complication rates etc.

Topic previously audited and requiring re-audit

Wide variation in practice

$\checkmark$ Risk to staff or patients/consumers identified

Topic concerned with high volume/high cost

Other (please describe): data not routinely collected

\subsection{Audit Details}

Audit title: Kangaroo Care practice audit

Background to audit: Kangaroo Care is one core modality of developmental care for sick or premature babies who are being medically managed in hospital. There is

Form: Clinical audit registration and proposal form Page 1

\section{7/08/2015}

Regard printed versions of this document as out of date - The current version 
Document Facilitator: Clinical Audit Advisor Senior Document Owner: Quality and Risk Manager Document Number: Error! Unknown document property name. Issue Date: 15 January 2013

Review Date: 15 January 2016

scientific support for its implementation in physiologically stable infants bom of more than 27 weeks gestation and it is a developmentally appropriate intervention known to enhance some aspects of physiological, psychosocial and neurobehavioural development for infants and families. Its routine application is recommended by the World Health Organization in affluent and low income settings and all tertiary units in New Zealand have guidelines for Kangaroo Care practice.

Audit aim: To assess current practice of kangaroo care as indicated by guidelines and policy, through a retrospective case note study.

Audit objectives (should be specific, measurable, achievable, realistic and time-framed):

1. Measure current implementation rates of kangaroo care for eligible infants

2. Collect information on exclusion criteria for bables not eliglble for kangaroo care

3. Compare two months in the NICU, one being less busy than average and the other being more busy than average: the rationale is to highlight aspects of staff and institutional barriers to implementation eg understaffing, high room acuity etc.

Standards to be audited (may be derived from guidelines, policies, research, professional consensus, etc):

Standards are derived from the guideline document "Kangaroo Care", with practice being compared against policy guldelines for inclusion and exclusion criteria:

\section{Eligibility criteria:}

1. All babies receiving palliative care

2. Any infant greater than $\mathbf{2 7}$ weeks, (or less gestation and greater than $\mathbf{2}$ weeks old) and physiologically stable

3. Stable ventilated/Brainz monitored bables after case by case discussion with attending consultant/CNS/ACNM

4. Individual infant assessment for stability/suitability before starting each KC session; all ETT, CPAP tubing and IV lines secured prior to session

5. Babies who have a chest drain for $>2$ weeks after case by case discussion with attending consultant/CNS/ACNM

6. Assessment of availability of space is required

7. Assessment of acuity of room and potential admissions which could render the session stressful and inappropriate is required.

\section{Exclusion criteria:}

1. Infants less than 27 weeks gestation

2. Immediately prior to shift hand over

3. Umbilical and/or arterial lines; chest drains; therapeutic hypothermia treatment

4. Ventilated infants with known increased risk factors - medical advice against KC

5. If resuscitation is in progress in the room

6. When admitting a seriously ill infant in the room

7. If the baby is hypothermic - if mild, KC may increase the infants ternperature

8. Baby has high SBR and is under intensive phototherapy - if SBR is decreasing and is close to being 'out of range', a KC with a bill-blanket may be considered

Form: Clinical aydit registration and proposal form Page 2
Date printed from
$\begin{aligned} & \text { Regard printed versions of this document as out of date - The } \\ & \text { current version }\end{aligned}$


Document Facilitator: Clinical Audit Advisor Senlor Document Owner: Quality and Risk Manager Document Number: Errorl Unknown document property name. Issue Date: 15 January 2013
Review Date: 15 January 2016

\begin{tabular}{|l|l|}
\hline & $\begin{array}{l}\$ 3179.75 \text { mileage + parking } \\
\text { fund applied for October } 2015 \text { round }\end{array}$
\end{tabular}

8.0 Approval

What are the implications for the service if this audit is not approved? It may be important to measure and assess the quality of Kangaroo Care practice due to its implications for public health and long-term neurodevelopmental outcomes for babies born sick or preterm. An audit may provide avenues for further research, or result in a plan of action for change.

\subsection{Authorisation}

\subsection{Declaration}

- I confirm that the information supplied is accurate to the best of my knowledge.

- I agree to maintain staff and patient confidentiality.

- I agree to ensure this audit is completed, results disseminated, report and action plan completed in accordence with CCDHB Clinical Audlt Policy.

- In signing this form I agree to the above declarations.

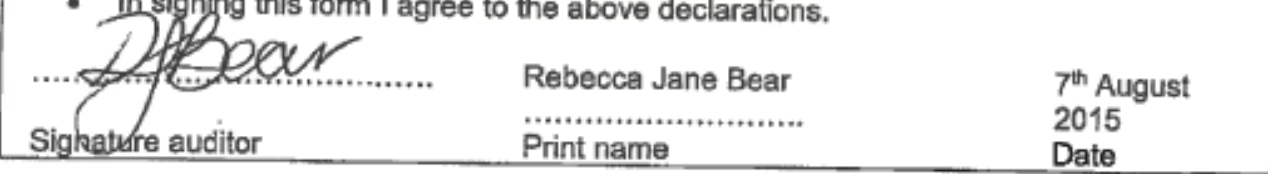

\subsection{Sign off}

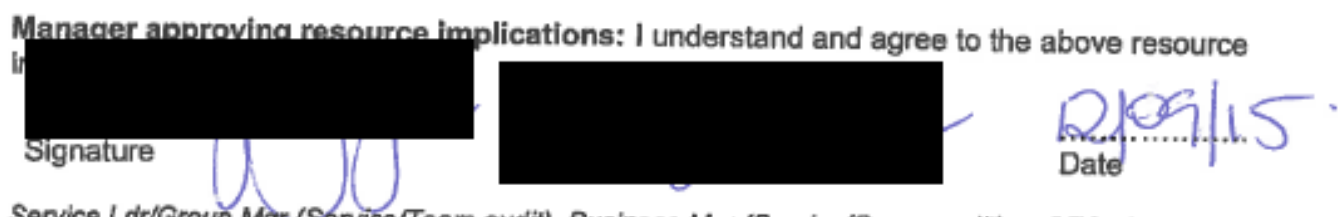
Service Ldr/Group Mgr-(Sonvice/Team audit), Business Mgr (Service/Group audit) or CEO, COO or
Director (Org/external audit)

Clinician/manager authorising clinical audit: I confirm that the implications and priority of this audit have been considered. I will ensure the dissemination of audit results and monitor the implemegtation of recommendations to obtain improvements in the quality of care

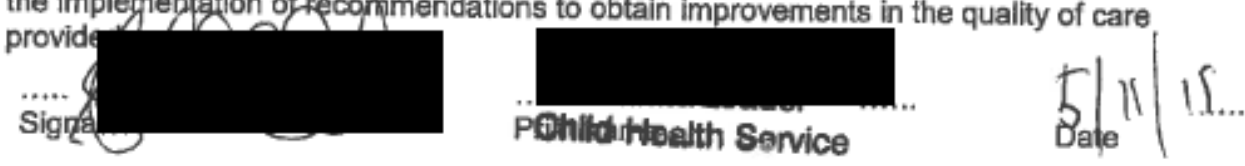
Clinical Lodr (Service/Team audit), Clinical Director (Service/Group audit) or CEO, COO or Director
(Org/external audit)

\begin{tabular}{l}
\hline Form: Clinical audit registration and proposal form Page 7 \\
Dale printed fiom \\
Regard printed verstrons of this document as out of date - The \\
current version
\end{tabular}




\section{Appendix 4: Human Ethics Committee Approval}

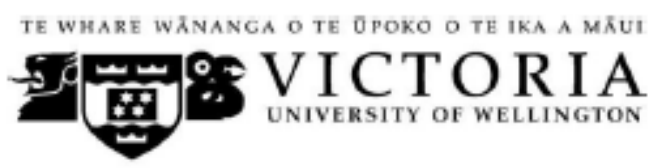

MEMORANDUM

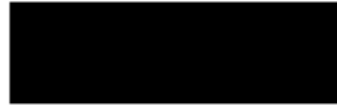

\begin{tabular}{l|l}
\hline TO & Rebecca Bear \\
\hline COPY TO & Dr Joan Skinner \\
\hline FROM & AProf Susan Corbett, Convener, Human Ethics Committee \\
\hline
\end{tabular}

\begin{tabular}{|c|c|}
\hline DATE & 28 July 2016 \\
\hline PAGES & 1 \\
\hline SUBJECT & $\begin{array}{l}\text { Ethics Approval: } 23008 \\
\text { Quality improvement of Kangaroo Mother Care in a Neonatal } \\
\text { Intensive Care Unit: A Participatory Action Research Project }\end{array}$ \\
\hline
\end{tabular}

Thank you for your application for ethical approval, which has now been considered by the Standing Committee of the Human Ethics Committee.

Your application has been approved from the above date and this approval continues until 31 December 2017. If your data collection is not completed by this date you should apply to the Human Ethics Committee for an extension to this approval.

Best wishes with the research.

Kind regards

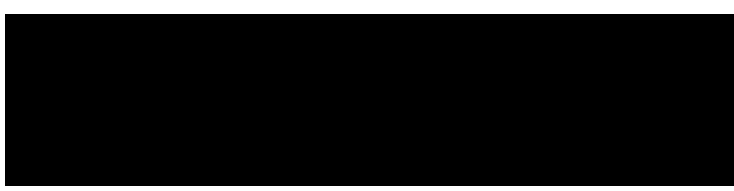

Susan Corbett

Convener, Victoria University Human Ethics Committee 


\title{
Appendix 5: Participant Information Sheet (Staff interviews, phase
}

2)

\author{
TE WHARE WÃNANGA O TE ÔPOKO O TE IKA A MÃUI \\ 59 \\ ㅂ.

\section{QUALITY IMPROVEMENT OF KANGAROO MOTHER CARE WITH PRETERM INFANTS IN ONE NICU IN AOTEAROA: $A$ participatory action research project}

\author{
INFORMATION SHEET FOR INTERVIEW PARTICIPANTS
}

Thank you for your interest in this project. Please read all information before deciding whether or not to take part. If you decide to participate, thank you. If you decide not to take part, thank you for considering my request.

Who am I?

My name is Rebecca Bear and I am a Doctoral student with the Graduate School of Nursing, Midwifery and Health at Victoria University of Wellington. This research project is work towards my thesis.

What is the aim of the project?

The aim of this project is to design a method for quality improvement and upscaling of Kangaroo Mother Care (KMC) of preterm infants in one Neonatal Intensive Care Unit (NICU) in Aotearoa. It uses a participatory action research approach.

This research has been approved by the Victoria University of Wellington Human Ethics Committee (Ethics approval number 23008)

How can you help?

If you agree to take part, I will interview you in a place of your choice, which could be somewhere in public, such as a café or library, or if you request it, within your workplace. I will ask you questions about institutional aspects of KMC in your setting. The interview will take 3060 minutes. I will record the interview and write it up later. You can stop the interview at any time, without giving a reason. You can withdraw from the study up to four weeks after the interview. If you withdraw, the information you provided will be destroyed or returned to you.

What will happen to the information you give?

This research is confidential. I will not name you in any reports, and I will not include any information that would identify you. A general descriptor of your role may be given as "healthcare practitioner", "management" or "both". As with all questions, this is optional. Only my supervisors and I will read the notes or transcript of the interview. The interview transcripts, summaries and any recordings will be kept securely and destroyed three years after the research ends. 
What will the project produce?

The information from my research will be used in my PhD thesis. You will not be identified in my report. I may also use the results of my research for conference presentations, and academic reports. I will take care not to identify you in any presentation or report.

If you accept this invitation, what are your rights as a research participant?

You do not have to accept this invitation if you don't want to. If you do decide to participate, you have the right to:

- choose not to answer any question;

- ask for the recorder to be turned off at any time during the interview;

- $\quad$ withdraw from the study up until four weeks after your interview;

- ask any questions about the study at any time;

- receive a copy of your interview recording (if it is recorded);

- read over and comment on a written summary of your interview;

- be able to read any reports of this research by emailing the researcher to request a copy.

If you have any questions or problems, who can you contact?

If you have any questions, either now or in the future, please feel free to contact either:

\section{Student:}

Name:

University email address:

\section{Supervisors:}

Name:

Role:

School:

Phone:

Email:

Name:

Role:

School:

Phone:

Email:

\section{Rebecca Bear}

rebecca.bear@vuw.ac.nz

\section{Dr Joan Skinner}

Senior Lecturer

Graduate School of Nursing, Midwifery and Health

joan.skinner@vuw.ac.nz

Dr Robyn Maude

Senior Lecturer

Graduate School of Nursing, Midwifery and Health

robyn.maude@vuw.ac.nz 


\section{APPENDICES}

\section{Human Ethics Committee information}

If you have any concerns about the ethical conduct of the research you may contact the Victoria University HEC Convener: Associate Professor Susan Corbett. Email susan.corbett@vuw.ac.nz or telephone+64-4-463 5480. 


\title{
Appendix 6: Participant Consent Form (Staff interviews, phase 2)
}

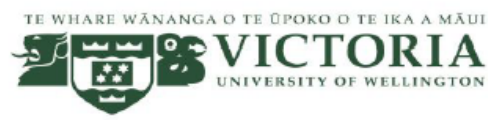 \\ QUALITY IMPROVEMENT OF KANGAROO MOTHER CARE WITH \\ PRETERM INFANTS IN ONE NICU IN AOTEAROA: \\ A participatory action research project \\ CONSENT TO INTERVIEW \\ This consent form will be held for five years.
}

Researcher: Rebecca Bear, Graduate School of Nursing, Midwifery and Health, Victoria University of Wellington.

- I have read the Information Sheet and the project has been explained to me. My questions have been answered to my satisfaction. I understand that I can ask further questions at any time.

- I agree to take part in an audio recorded interview.

I understand that:

- I may withdraw from this study until immediately prior to commencement of data analysis, and any information that I have provided will be returned to me or destroyed. I will be informed by the researcher when data analysis will begin.

- The information I have provided will be destroyed three years after the research is finished.

- $\quad$ Any information I provide will be kept confidential to the researcher and the supervisor. I understand that the results will be used for a $\mathrm{PhD}$ report and a summary of the results may be used in academic reports and/or presented at conferences.

- My name will not be used in reports, nor will any information that would identify me. The only role identification will be as either "health practitioner", "management" or "both".

AND

- I would like a copy of the transcript of my interview:

Yes $\square \quad$ No

- I would like a summary of my interview:

Yes $\square \quad$ No

- $\quad$ I would like to receive a copy of the final report and have added my Yes $\square$ No email address below.

Signature of participant:

Name of participant:

Date:

Contact details: 


\section{Appendix 7: Staff Interview Guide (phase 2)}

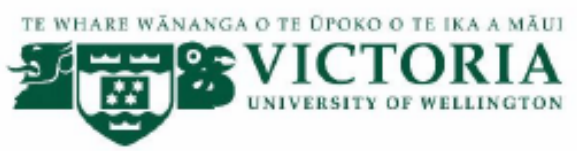

QUALITY IMPROVEMENT OF KANGAROO MOTHER CARE OF PRETERM INFANTS IN ONE NICU IN AOTEAROA: A participatory action research project

\section{INTERVIEW QUESTIONS FOR NICU HEALTHCARE PRACTITIONERS AND MANAGERS}

1. What category does your NICU role fit into: healthcare professional, manager, educator?

2. What are your thoughts about Kangaroo Mother Care? It's importance for the babies and families on the unit?

3. "The basics" of the environment: Lighting, sound, physical space, privacy, quiet time....

4. Are there certain characters who act as champions - or is it an even spread? Certain profession-types?

5. Tell me about the barriers to KMC: parental, staff, workplace, organizational

6. Do you believe $\mathrm{KC}$ needs to be upscaled in this hospital? What, if any, changes would you make to $\mathrm{KC}$ education and practice? Are you aware of any gaps between knowledge and practice?

7. How do things get done? Who supports you? How is change introduced, implemented and sustained?

8. Tell me about the general culture of continued quality improvement and change in your workplace?

9. What strategies would be helpful prior to the start of a project to upscale KMC? What measures do you think would be helpful in increasing and sustaining KMC practice? These could include planning, education, advertising KMC, policy-making etc Are extra resources required? These may be physical eg chairs; supportive eg staff mentoring; educational eg parent "learn at lunch" meetings

10. I'm interested in doing a small quantitative study of $K C$ on the unit. What would you like me to do to help facilitate a KMC project? Can you suggest any areas for investigation?

11. Anything else to add? 


\section{Appendix 8: Human Ethics Committee Approval, Amendment 1}

Te whare wananga o te opoko o te ika a mát

MEMORANDUM

\begin{tabular}{l|l}
\hline TO & Rebecca Bear \\
\hline COPY TO & Joan Skinner \\
\hline FROM & AProf Susan Corbett, Convener, Human Ethics Committee \\
\hline
\end{tabular}

\begin{tabular}{l|l}
\hline DATE & 24 February 2017 \\
\hline PAGES & 1 \\
\hline
\end{tabular}

\begin{tabular}{l|l}
\hline SUBJECT & $\begin{array}{l}\text { Ethics Approval: } 23008 \\
\text { Quality improvement of Kangaroo Mother Care in a Neonatal } \\
\text { Intensive Care Unit: A Participatory Action Research Project }\end{array}$ \\
\hline
\end{tabular}

Thank you for your request to amend your ethics approval. This has now been considered and the request granted.

Your application has approval until 31 December 2017. If your data collection is not completed by this date you should apply to the Human Ethics Committee for an extension to this approval.

Best wishes with the research.

Kind regards

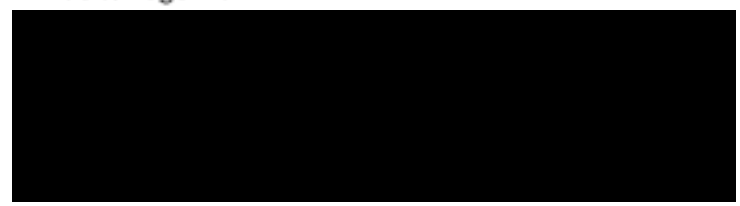

Susan Corbett

Convener, Victoria University Human Ethics Committee 


\section{Appendix 9: Human Ethics Committee Approval, Amendment 2}

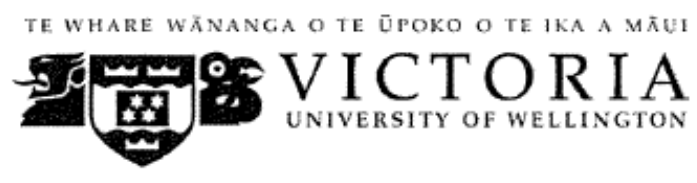

MEMORANDUM

Phone

Email

\begin{tabular}{l|l}
\hline TO & Rebecca Bear \\
\hline COPY TO & Joan Skinner \\
\hline FROM & Dr Averil Coxhead, Acting Convener, Human Ethics Committee \\
\hline
\end{tabular}

\begin{tabular}{l|l}
\hline DATE & 29 May 2017 \\
\hline PAGES & 1 \\
\hline
\end{tabular}

\begin{tabular}{l|l}
\hline SUBJECT & $\begin{array}{l}\text { Ethics Approval: } 23008 \\
\text { Quality improvement of Kangaroo Mother Care in a Neonatal } \\
\text { Intensive Care Unit: A Participatory Action Research Project }\end{array}$ \\
\hline
\end{tabular}

Thank you for your request to amend your ethics approval. This has now been considered and the request granted.

Your application has approval until 31 December 2017. If your data collection is not completed by this date you should apply to the Human Ethics Committee for an extension to this approval.

Best wishes with the research.

Averil Coxhead,

Acting Convener, Victoria University Human Ethics Committee 


\section{Appendix 10: Institutional Approval KMC Observation (phase 3)}

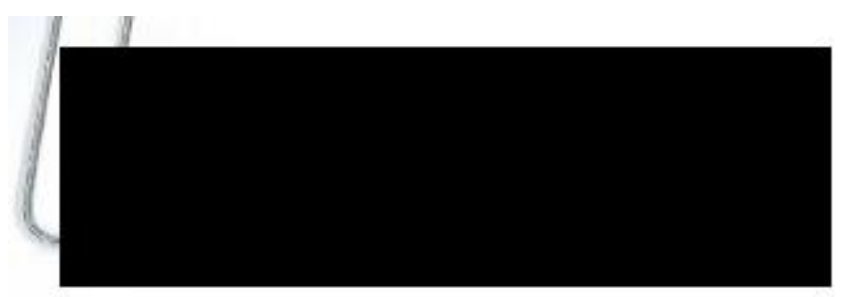

Child Health Governance Group Research Locality Assessment Form

Name of principal investigator: Rebecca Bear

Name of associated institution: Vuctura univesity

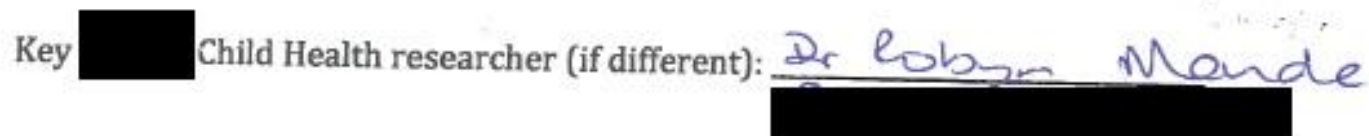

Title of Project: Obsen vat on of Kancenvo mothe

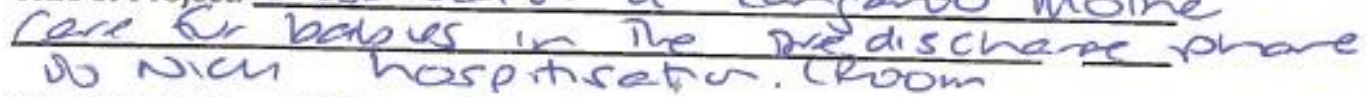
Date discussed: tots 313117

This project has been considered by the Child Health Governance Group and approval given for the research to be located in Child Health. Please send a copy of the final results of your research project or audit to the Child Health Quality Facility and Research Office.

Chair Governance Group:

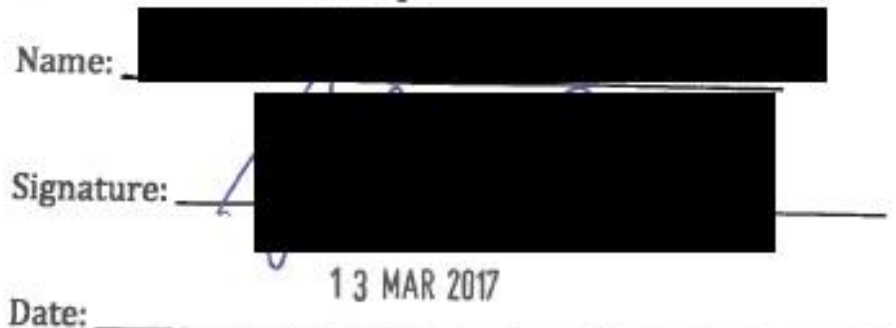

Date:

3 MAR 2017
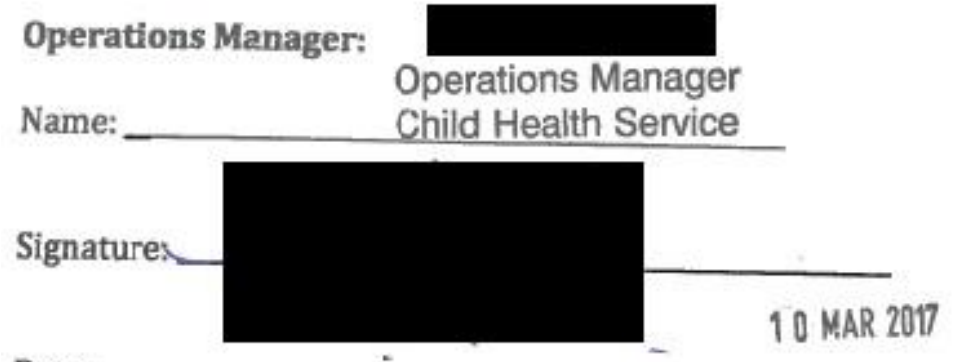

Date: 


\title{
Appendix 11: Staff Information Sheet (KMC observation, phase 3)
}

\author{
TE WHARE WÃNANGA O TE ÓPOKO O TE IKA A MÃUI \\ S2:

\section{OBSERVATION OF KANGAROO MOTHER CARE OF BABIES PRIOR TO DISCHARGE FROM THE NICU \\ $A$ PhD research project}

\author{
INFORMATION SHEET FOR NICU STAFF
}

\section{Who am I?}

My name is Rebecca Bear and I am a Doctoral student with the Graduate School of Nursing, Midwifery and Health at Victoria University of Wellington. As you may be aware, for the last 18 months I have been working in collaboration with staff in the NICU on research involving Kangaroo mother care (KMC), with the aim of understanding this practice more fully within the unit.

Previously, individual interviews with some NICU staff members identified a possible gap in practice of KMC with infants in the pre-discharge phase of hospitalization. This information sheet is to inform you of my intended observation in Room A, as part of a KMC quality assurance project.

What is the aim of the project?

The aim of this project is to evaluate to what extent KMC is being implemented for babies in including potential enablers and barriers to the practice. For this purpose I will observe and record the babies' experience of Kangaroo Mother Care (KMC) in the predischarge phase of hospitalization. Data collected will focus on the day-to-day experience of babies within this setting, including the frequency and duration of KMC (if any), number of caregivers involved and the type of handling, feeding and care the babies experience. I will also collect characteristics of the room, such as sound, lighting and space/privacy. No video or sound recordings will be taken.

This research is part of a larger KMC project which began in the NICU in July, 2016 and was approved by Victoria University of Wellington Human Ethics Committee [\#23008; 24.2.2017, amendment], a second cycle of research following the original application [\#23008;

24.7.2016]. This next cycle of research was designed in collaboration with NICU staff who participated in an interview around the strengths, weaknesses, opportunities and threats to KMC practice within your unit. A finding from the initial cycle of research identified a possible gap in KMC practice for babies prior to discharge from the unit and is supported by a group of staff including your team leader, 


\section{How can you help?}

Data collection for this project will not directly impact on your day-to-day practice and I will not be focusing any observation on staff practice, as such. The focus of data collection will be on the direct experience of KMC for the babies, other types of care including feeding and handling, and the characteristics of the environment of However, I acknowledge that the room is part of your workplace and I provide assurance that aspects of staff practice which is indirectly observed will not be collected as data and will remain confidential. Once analysed, a report including preliminary findings will be available to staff and fed back to the staff group by arrangement.

\section{What will happen to the information collected?}

This research is confidential. Nobody will be named in any report, and I will not include any information that would identify you. Only my supervisors and I will read the data and research notes. The data and summaries will be kept securely and destroyed three years after the research ends.

\section{What will the project produce?}

The information from my research will be used to inform care in the NICU. It will also be used as part of my PhD thesis. I may also use the results of my research for conference presentations, and academic reports. Care will be taken not to identify either participants (babies), the staff members who care for them, or the workplace in any presentation, report or in my thesis.

\section{Research participation}

As this project is focused on the experience of the babies they are considered the participants. No data of any baby will be used until consent has been obtained by the baby's legal guardian. I will have no contact with anyone in the room except for obtaining guardian consent.

If you have any questions or problems, who can you contact?

If you have any questions, either now or in the future, please feel free to contact either:

NICU manager and research partner

Name

Work email address:

\section{Student:}

Name:

University email address:

Phone

\section{Research Supervisors:}

Rebecca Bear

rebecca.bear@vuw.ac.nz 


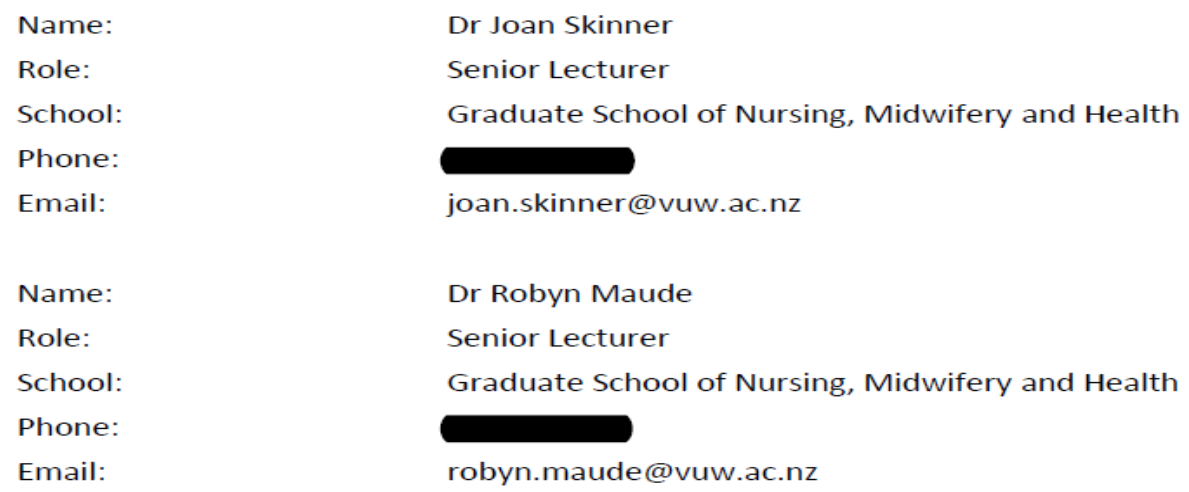

Email:

robyn.maude@vuw.ac.nz

\section{Human Ethics Committee information}

If you have any concerns about the ethical conduct of the research you may contact the Victoria University HEC Convener: Associate Professor Susan Corbett. Email susan.corbett@vuw.ac.nz or telephone +64-4-463 5480.

\section{Health and Disability Ethics Committee information}

I you want to talk to someone who isn't involved with the study, you can contact an independent health and disability advocate on:

Phone:

0800555050

Fax: O800 2 SUPPORT (080027877678)

Email: advocacy@hdc.org.nz

You can also contact the health and disability ethics committee (HDEC) that approved this study on:

Phone:

08004 ETHICS

Email:

hdecs@moh.govt.nz 


\title{
Appendix 12: Parent Information Sheet (KMC observation, phase 3)
}

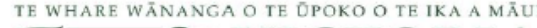 \\ 59 \\ (M) UNIVERIIY OF WELLINGTON

\section{OBSERVATION OF KANGAROO MOTHER CARE OF BABIES PRIOR TO DISCHARGE FROM THE NICU $A$ PhD research project}

\author{
INFORMATION SHEET FOR KANGAROO MOTHER CARE OBSERVATION IN
}

Thank you for your interest in this project. Please read all information before deciding whether or not to take part. If you decide to consent to my observation of your baby's experience, thank you. If you decide not to take part, thank you for considering my request.

Who am I?

My name is Rebecca Bear and I am a Doctoral student with the Graduate School of Nursing, Midwifery and Health at Victoria University of Wellington. This research project is work towards my thesis. This study was approved by Victoria University of Wellington Human Ethics Committee [\#23008; 24.2.2017, amendment], a second cycle of research following the original application [\#23008; 24.7.2016].

\section{What is the aim of the project?}

The purpose of this phase of the project is to observe Kangaroo Mother Care (KMC) and your baby's experience of it, if any, leading up to your infants discharge from the Neonatal Intensive Care Unit (NICU). I will be observing in_or two weeks and taking notes specific to Kangaroo Mother Care and aspects of including layout, sound and lighting. There will be no video or sound recording taken as part of my observation. This study is part of a larger project being conducted in partnership with the NICU staff to understand Kangaroo Mother Care in the NICU more fully. It began in July 2016.

\section{How can you help?}

If you agree to the observation of your baby's experience, he or she will be observed by me over a period of two weeks. The observation period will last no longer than 8 hours per day. The information recorded will include what type of handling, feeding and care she or he received, including whether Kangaroo Mother Care was part of your baby's day. I will also collect information about the number of babies and adults that come and go in the room and sound, lighting and space/privacy levels. The identity of your baby will remain anonymous and protected. I will collect the data, analyse it and produce a report of general findings. You can stop the recording of the observations at any time, without giving a reason. You can withdraw from the study up to two weeks after the final day of observation. If you withdraw, the information collected about your baby will be destroyed. 


\section{APPENDICES}

\section{What will happen to the information that is collected?}

This research is confidential. Your baby will not be named in any reports, and I will not include any information that would identify your baby. Only my supervisors and I will read the recordings. The data will be kept securely and destroyed three years after the research ends.

\section{What will the project produce?}

The information from my research will be used in my PhD thesis. I may also use the results of my research for conference presentations, and academic reports. I will take care not to identify your baby in any presentation, report or in my thesis.

\section{If you accept this invitation, what are your rights as a research participant?}

You do not have to accept this invitation if you don't want to. If you do decide to allow your baby to be observed, you have the right to:

- $\quad$ ask for the observation to cease at any given time;

- $\quad$ withdraw from the study up until two weeks after the observation ends;

- ask any questions about the study at any time;

- $\quad$ receive a copy of the observational notes directly relating to your baby;

- be able to read any reports regarding this research by emailing the researcher to request a copy.

If you have any questions or problems, who can you contact?

If you have any questions, either now or in the future, please feel free to contact either:

\section{Student:}

Name:

Rebecca Bear

University email address:

rebecca.bear@vuw.ac.nz

Phone:

\section{Supervisors:}

Name:

Dr Joan Skinner

Role:

Senior Lecturer

School:

Graduate School of Nursing, Midwifery and Health

Phone:

Email:

joan.skinner@vuw.ac.nz

Name:

Dr Robyn Maude 


\title{
Appendix 13: Participant Consent Form (KMC observation, phase
}

3)

\author{
TE WHARE WANANGA O TE OPOKO O TE IKA M MX̃U

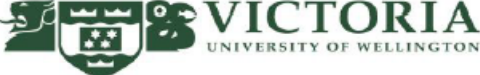 \\ OBSERVATION OF KANGAROO MOTHER CARE AND THE DAY-TO-DAY \\ EXPERIENCE OF BABIES PRIOR TO DISCHARGE FROM THE NICU \\ $A P h D$ research project
}

CONSENT FOR OBSERVATION

This consent form will be held for five years.

Researcher: Rebecca Bear, Graduate School of Nursing, Midwifery and Health, Victoria University of Wellington.

- I have read the Information Sheet and the project has been explained to me. My questions have been answered to my satisfaction. I understand that I can ask further questions at any time.

- I agree to my baby being observed and data collected about his/her experience of Kangaroo Mother Care and other aspects of feeding and handling whilst in

I understand that:

- I may withdraw consent for my baby to be observed for this study until two weeks after the end of data collection and any information that has been collected will be returned to me or destroyed. I will be informed by the researcher when data collection has finished.

- The information I have provided will be destroyed three years after the research is finished.

- Any information I provide will be kept confidential to the researchers and the supervisors. I understand that the results will be used for a PhD report and a summary of the results may be used in academic reports and/or presented at conferences.

- My name or my baby's name will not be used in reports, nor will any information that would identify me.

AND

I would like a copy of the data collected from observation of my baby: $\quad$ Yes $\square$ No $\square$

I would like to receive a copy of the final report and have added my Yes $\square$ No $\square$ email address below.

Signature of participant:

Name of participant:

Date:

Contact details: 
Appendix 14: KMC Observation Tool (phase 3)

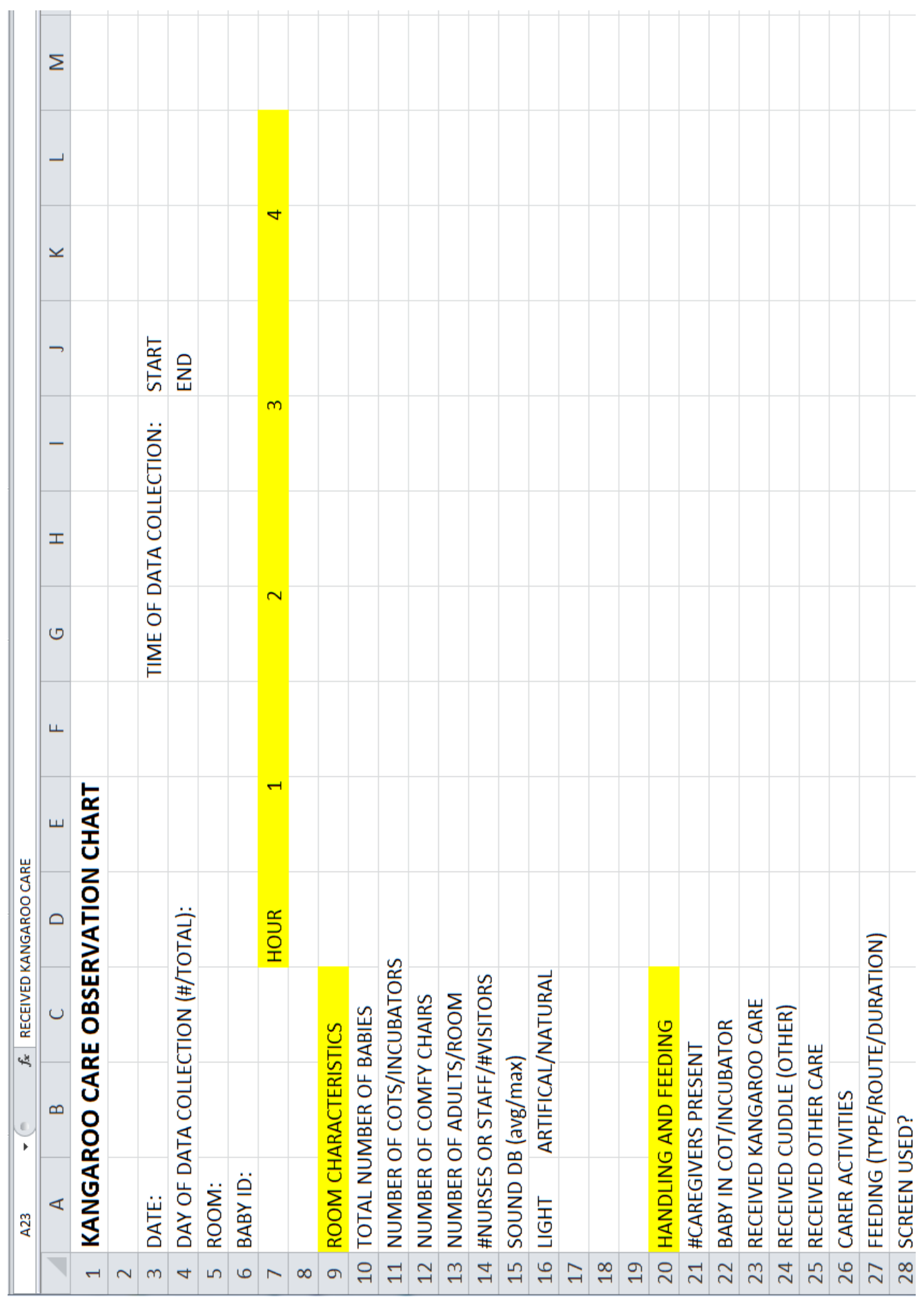




\section{Appendix 15: Human Ethics Committee Approval, Amendment 3}

TE WHARE WR̃NANGA O TE ŌPOKO O TE IKA A MKUUI

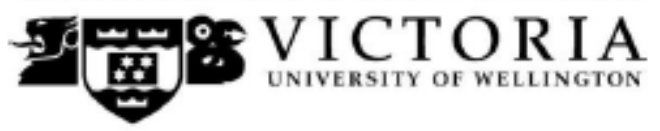

MEMORANDUM

\begin{tabular}{l|l}
\hline TO & Rebecca Bear \\
\hline COPY TO & Joan Skinner \\
\hline FROM & Dr Averil Coxhead, Acting Convener, Human Ethics Committee \\
\hline DATE & 29 May 2017 \\
\hline PAGES & 1 \\
\hline & $\begin{array}{l}\text { Ethics Approval: 23008 } \\
\text { Quality improvement of Kangaroo Mother Care in a Neonatal } \\
\text { Intensive Care Unit: A Participatory Action Research Project }\end{array}$ \\
\hline
\end{tabular}

Thank you for your request to amend your ethics approval. This has now been considered and the request granted.

Your application has approval until 31 December 2017. If your data collection is not completed by this date you should apply to the Human Ethics Committee for an extension to this approval.

Best wishes with the research.

Averil Coxhead,

Acting Convener, Victoria University Human Ethics Committee 


\title{
Appendix 16: Participant Information (Parent interview, phase 4)
}

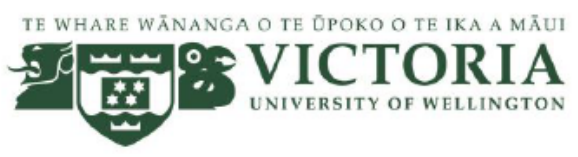

\section{PARENTAL EXPERIENCE OF KANGAROO MOTHER CARE IN THE NICU $A$ PhD research project}

\author{
INFORMATION SHEET FOR KANGAROO MOTHER CARE INTERVIEW
}

Thank you for your interest in this project. Please read all information before deciding whether or not to take part. If you decide to consent to interview about your experience of Kangaroo Mother Care while your baby was in hospital, thank you. If you decide not to take part, thank you for considering my request.

\section{Who am I?}

My name is Rebecca Bear and I am a Doctoral student with the Graduate School of Nursing, Midwifery and Health at Victoria University of Wellington. This research project is work towards my thesis. This study was approved by Victoria University of Wellington Human Ethics Committee [\#23008; $29^{\text {th }}$ May 2017, amendment \#3], a third cycle of research following the original application [\#23008; 24.7.2016].

\section{What is the aim of the project?}

The purpose of this phase of the project is to hear from parents about their experience of Kangaroo Mother Care (KMC) while their baby has been in the Neonatal Intensive Care Unit (NICU). I have collected data from the organisation and from the staff and I am now interested in the perspectives of the parents. This study is part of a larger project being conducted in partnership with the NICU staff to understand Kangaroo Mother Care in the NICU more fully. It began in July 2016.

\section{How can you help?}

If you agree to an interview about your experience of Kangaroo Mother Care, it will take place at a time and place that suits you. I will make a sound recording of our interview, which will be partly transcribed and given back to you for your feedback before publishing in my thesis. The information recorded may include some of your thoughts, feelings, experiences and beliefs about Kangaroo Mother Care which are relevant to you. The identity of you, your whanau/family and your baby will remain anonymous and protected. You may stop the recording at any time, without giving a reason. You can withdraw from the study up to four weeks after the interview. If you withdraw, the information collected from you will be destroyed. 


\section{What will happen to the information that is collected?}

Your identity and that of your whanau/family and/or baby will be protected and not named in any reports, nor will it include any information that would identify you all. Only my supervisors and I will read the recording transcript. The data will be kept securely and destroyed three years after the research ends.

\section{What will the project produce?}

The information from my research will be used in my PhD thesis. I will also use the results of my research for conference presentations, and academic reports. I will take care not to identify you, your whanau/family and/or baby in any presentation, report or in my thesis.

\section{If you accept this invitation, what are your rights as a research participant?}

You do not have to accept this invitation if you don't want to. If you do decide to interview, you have the right to:

- ask for the interview to cease at any given time;

- $\quad$ withdraw from the study up until four weeks after the interview ends;

- $\quad$ ask any questions about the study at any time;

- receive a copy of the interview recording and notes directly relating to you and your baby;

- be able to read any reports regarding this research by emailing the researcher to request a copy.

If you have any questions or problems, who can you contact?

If you have any questions, either now or in the future, please feel free to contact either:

\section{Student:}

Name:

Rebecca Bear

University email address:

rebecca.bear@vuw.ac.nz

Phone:

\section{Supervisors:}

Name:

Dr Joan Skinner

Role:

\section{Senior Lecturer}

School:

Graduate School of Nursing, Midwifery and Health

Phone:

Email:

joan.skinner@vuw.ac.nz

Name:

Dr Robyn Maude

Role:

Senior Lecturer 
School: Graduate School of Nursing, Midwifery and Health

Phone:

Email:

robyn.maude@vuw.ac.nz

\section{Human Ethics Committee information}

If you have any concerns about the ethical conduct of the research you may contact the Victoria University HEC Convener: Associate Professor Susan Corbett. Email susan.corbett@vuw.ac.nz or telephone+64-4-463 5480.

\section{Health and Disability Ethics Committee information}

I you want to talk to someone who isn't involved with the study, you can contact an independent health and disability advocate on:

Phone: $\quad 0800555050$

Fax: $\quad 08002$ SUPPORT (080027877678)

Email: $\quad$ advocacy@hdc.org.nz

You can also contact the health and disability ethics committee (HDEC) that approved this study on:

Phone:

08004 ETHICS

Email:

hdecs@moh.govt.nz 


\section{Appendix 17: Participant Consent Form (Parent interview, phase 4)}

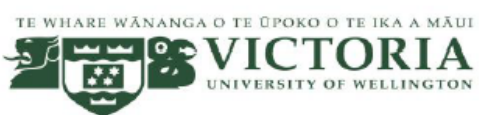

\section{PARENTAL EXPERIENCE OF KANGAROO MOTHER CARE THE NICU $A P h D$ research project}

\section{CONSENT FOR KANGAROO MOTHER CARE INTERVIEW}

This consent form will be held for five years.

Researcher: Rebecca Bear, Graduate School of Nursing, Midwifery and Health, Victoria University of Wellington.

- I have read the Information Sheet and the project has been explained to me. My questions have been answered to my satisfaction. I understand that I can ask further questions at any time.

- I agree to being interviewed about my experience of Kangaroo Mother Care while my baby was hospitalized in the NICU

I understand that:

- I may withdraw consent for the use of my interview material until four weeks after the end of my interview and any information that has been collected will be returned to me or destroyed. I will be informed by the researcher when data collection has finished, upon request.

- $\quad$ The information I have provided will be destroyed three years after the research is finished.

- Any information I provide will be kept confidential to the researchers and the supervisors. I understand that the results will be used for a PhD report and a summary of the results may be used in academic reports and/or presented at conferences.

- My name, my family or baby's name will not be used in reports, nor will any information that would identify me.

AND

- $\quad$ I would like a copy of the sound recording and partial transcript of my Yes $\square$ No interview

- I would like to receive a copy of the final report and have added my Yes $\square$ No email address below.

Signature of participant:

Name of participant:

Date:

Contact details: 


\title{
Appendix 18: Parent Interview Guide (phase 4)
}

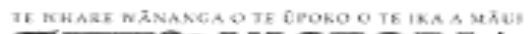 \\ 하요 VICTORIA

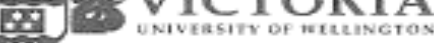 \\ PARENTAL EXPERIENCE OF KANGAROO MOTHER CARE IN THE NICU \\ $A$ PhD research project \\ INTERVIEW QUESTIONS FOR NICU PARENTS
}

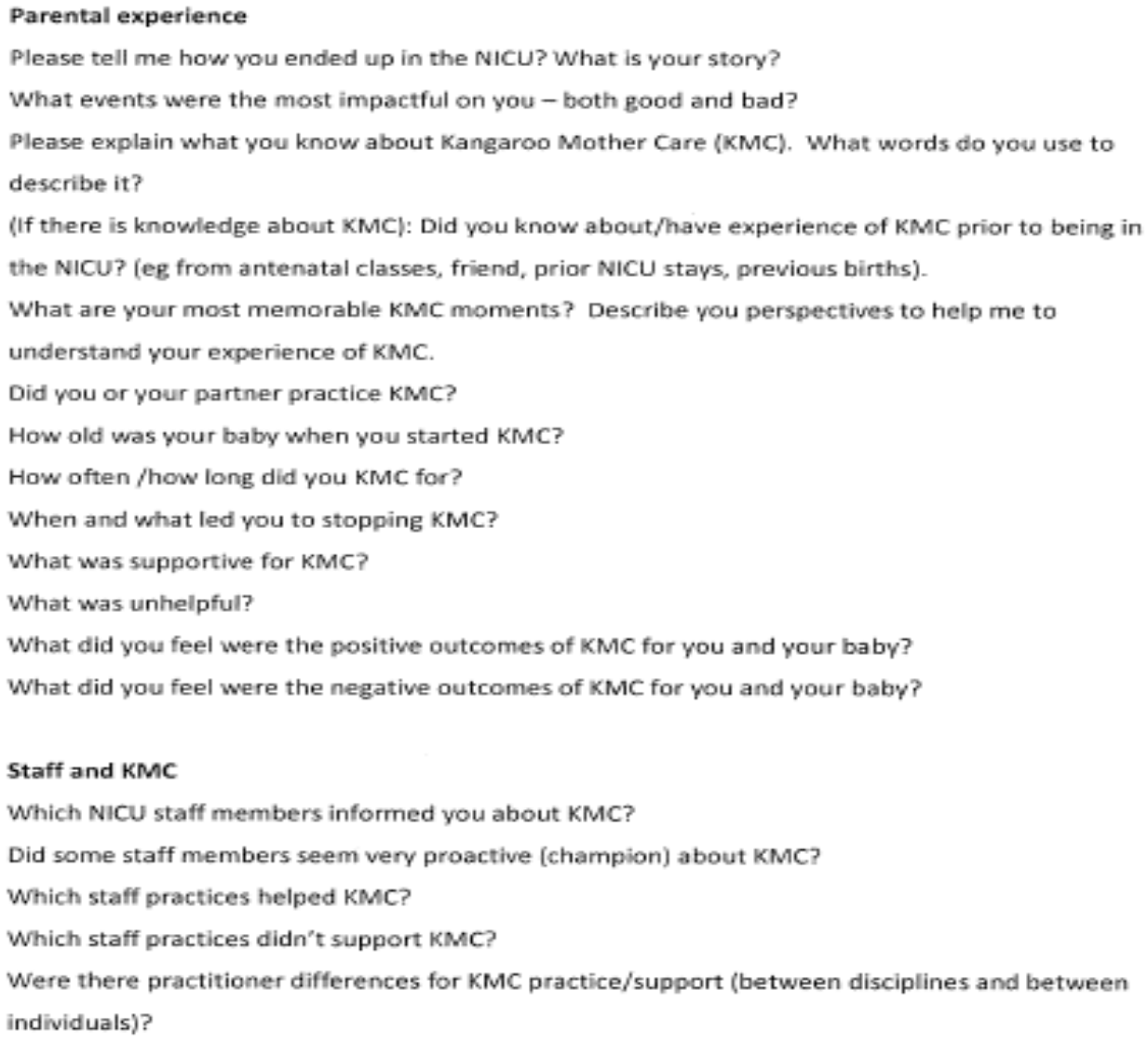




\section{Appendix 19: PAR-Participant Interview Guide (phase 5)}

\section{[abridged]}

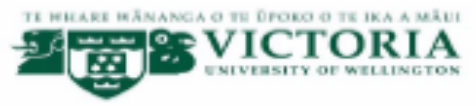

\section{NARRATIVE INTERVIEW: REFLECTIONS ON A KMC PROJECT $A$ PhD research project}

\section{INTERVIEW GUIDE FOR PAR PARTICIPANT: FINAL INTERVIEW}

1. Firstly, please share with me your most notable perspectives about the NICU summary report.

2. Tell me about what stands out for you in the findings of the NICU report?

3. What are your thoughts (if any) about participatory action research for this KMC study?

4. Do you have any advice or perspectives a bout my performance as a researcher?

5. Are there ways that you can think of which could have enhanced the procesa?

6. Are you prompted by this research process or the findings in the report to consider doing more KMC research?

7. Apart from the findings that were reported in the research summary, 1 have performed a discourse analysis of my own field note observations and other relevant texts (case note documentation, KMC policy guideline, staff and parent interviews, observational study etc).

8. From what I understand to be predominating discourses within the NICU, I have identified the following five categories and have given some examples. Would you be willing to comment with your understanding of these?

a. Enduring historical discourses: physician-centrism and paternalism; biomedical and technological hegemony (dominance); sickness (treatment) vs wellness (prevention) opinionvpersonality-based care

(give examples)

b. Persistent hierarchical organisational style: medical profession-caring profession divide; patriarchal ideology; nurse-led care; parents self-regulating their interactions with professionals to protect good care of their babies (give examples)

c. Baby as patient, not person: ownership of babies by staff especially nurses; baby considered sick, in requirement of nursing, not parenting (give examples)

d. Parent as outsider; potential source of difficult emotions and 'messiness' (give examples)

e. Paradigm-change discourse, not yet embedded in practice (give examples) 


\section{Appendix 20: Health and Disability Ethics Committee, Approval 1}

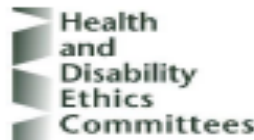

Victorla University of Wellington

Dear Ms Bear,
Health and Disability Ethics Committees 20 Ritien Btret Feybeg Bulding
PO Bex 5013

Wellingtion

08004 ETHICS

Study title:

Quality Improvement of Kanqaroo Mother Care of preterm infants in a Neonatal Intensive Care Unit in Aotearoa

Thank you for emalling HDEC a completed scope of revlew form on 26 April 2016, and providling clarification on 3 May 2016. The Secretarlat has assessed the information provided In your form and supporting documents against the Standard Operating Procedures.

Your study will not require submission to HDEC, as on the basis of the informaton you have submitted, It does not appear to be within the scope of HDEC review. This scope is described in section three of the Standard Operating Procedures for Health and Disability Ethics Commiltees.

An observational study requires HDEC review only if the study involves more than minimal risk (that is, potential participants could reasonably be expected to regard the probability and magnitude of possible harms resurting from their particlpation in the study the study).

Your study does not involve human participants recrulted in their capacity as:

- consumers of health or disablity support services, or

- relatlves or careglvers of consumers of health or dlsabllty support services, or volunteers in cllnical trials (Including, for the avoldance of doubt, bioequivalence and bioavallability studies;

The project also involves quality improvement. Any health Information used will fall under audit. An audit or related activity requires HDEC review only if it involves the use, collection or storage of human fissue without consent, other than in accordance with statutory exception (set out at section $20(1)$ of the Human Tissue Act 200,8 and Right
$7(10)(\mathrm{c})$ of the Code of Hearth and Disability Services Consumers' Riqhts 1996)-

If you consider that our advice on your project being out of scope is in incorrect please contact us as soon as possible giving reasons for this.

This letter does not constitute ethical approval or endorsement for the activity described In your application, but may be used as evidence that HDEC review is not required for it.

Please note, your locality may have additional ethical revlew pollcles, please check with your locality. If your study involves a DHB, you must contact the DHB's research ofnce before you begin. If your study involves a university or polytechnic, you must contact its Institutional ethics committee before you begin.

Out or Scope - HOEC email suberibat

Please dont hesitate to contact us for further information.

Yours sincerely. 


\section{REFERENCES}

Aagaard, H., \& Hall, E. O. C. (2008). Mothers' experiences of Having a Preterm Infant in the Neonatal Care Unit: A Meta-Synthesis. Journal of Pediatric Nursing, 23(3), e26-e36.

Abadia-Barrero, C. E. (2018). Kangaroo Mother Care in Colombia: A Subaltern Health Innovation Against For-Profit Biomedicine. Medical Anthropology Quarterly. https://doi.org/10.1111/maq.12430.

Abel, S., Park, J., Tipene-Leach, D., Finau, S., \& Lennan, M. (2001). Infant care practices in New Zealand: a cross-cultural qualitatitive study. Social Science \& Medicine, 53, 1135-1148.

Adkins, C. S., \& Doheny, K. K. (2017). Exploring Preterm Mothers' Personal Narratives. Influences and Meanings. Advances in Nursing Science, 40(2), 154-167. doi:10.1097/ANS.0000000000000150.

Aguggia, J. P., Suarez, M. M., \& Rivarola, M. A. (2013). Early maternal separation: Neurobehavioral consequences in mother rats. Behavioural Brain Research, 248, 25-31. http://dx.doi.org/10.1016/j.bbr.2013.03.040.

Ahern, C., McKinnon, M. C., Bieling, P. J., McNeely, H., \& Langstaff, K. (2016). Overcoming the Challenges Inherent in Conducting Design Research in Mental Health Settings: Lessons from St. Joseph's Healthcare, Hamilton's Pre and Post-Occupancy Evaluation. Health Environments Research \& Design Journal, 9(2), 119-129. doi:10.1177/1937586715602219.

Alianmoghaddam, N., Phibbs, S., \& Benn, C. (2017). Resistance to breastfeeding: A Foucauldian analysis of breastfeeding support from health professionals. Women and Birth, 30, e281-e291. http://dx.doi.org/10.1016/j.wombi.2017.05.005.

Aloysius, A., Platonos, K., Theakstone-Owen, A., Deierl, A., \& Banerjee, J. (2018). Integrated family delivered care: Development of a staff education programme. Journal of Neonatal Nursing, 24, 35-38. https://doi.org/10.1016/j.jnn.2017.11.009.

Als, H. (1982). Toward a Synactive Theory of Development: Promise for the Assessment and Support of Infant Individuality. Infant Mental Health Journal, 3(4), 229-243.

Als, H., Duffy, F., H., McAnulty, G., B., Rivkin, M., J, U., Vajapeyam, S., Mulkern, R., V., Eichenwald, E., C. (2004). Early Experience Alters Brain Function and Structure. Pediatrics, 113, 846-857.

Als, H., McAnulty, G., \& Gloria, B. (2011). The Newborn Individualized Developmental Care and Assessment Program (NIDCAP) with Kangaroo Mother Care (KMC): Comprehensive Care for Preterm Infants. Current Women's Health Reviews, 7(3), 288-301.

Altimier, L. (2015). Neuroprotective Core Measure 1: The Healing NICU Environment. Newborn \& Infant Nursing Reviews, 15, 91-96. http://dx.doi.org/10.1053/j.nainr.2015.06.014.

Altimier, L., Kenner, C., \& Damus, K. (2015). The Wee Care Neuroprotective NICU Program (Wee Care): The Effect of a Comprehensive Developmental Care Training Program on Seven Neuroprotective Core Measures for FamilyCentred Developmental Care of Premature Neonates. Newborn \& Infant Nursing Reviews, 15, 6-16. 
Altimier, L., \& Phillips, R. (2016). The Neonatal Integrative Developmental Care Model: Advanced Clinical Applications of the Seven Core Measures for Neuroprotective Family-centered Develpomental Care. Newborn \& Infant Nursing Reviews, 16, 230-244. http://dx.doi.org/10.1053/j.nainr.2016.09.030.

Altimier, L., \& Phillips, R., M. (2013). The Neonatal Integrative Developmental Care Model: Seven Neuroprotective Core Measures for Family-Centered Developmental Care. Newborn \& Infant Nursing Reviews, 13, 9-22. doi:10.1053/j.nainr.2012.12.002

Altman, M., Kantrowitz-Gordon, I., \& Vandermause, R. (2014). Synergy Among Multiple Methodologies: Investigation Parents' Distress After Preterm Birth. International Journal of Qualtitative Methods, 13, 335-346.

Alves, E., Rodrigues, C., Fraga, S., Barros, H., \& Silva, S. (2013). Parents' Views on Factors that Help or Hinder Breast Milk Supply in Neonatal Care Units: Systematic Review. Archives of Disease in Childhood. Fetal and Neonatal Edition, 98, F511-517. doi: 10.1136/archdischild-2013-304029.

American Academy of Pediatrics. (2012a). Early Childhood Adversity, Toxic Stress, and the Role of the Pediatrician: Translating Developmnetal Science Into Lifelong Health. Pediatrics. doi:10.1542/peds.2011-2662.

American Academy of Pediatrics. (2012b). Policy statement: Patient- and familycentered care and the Pediatrican's role. Pediatrics, 120(2).

American Academy of Pediatrics, Council of International Neonatal Nurses, International Council of Nurses, American College of Obstetricians and Gynecologists, International Federation of Gynecology and Obstetrics, \& Nurse-Midwives, A. C. o. (2016). JOINT STATEMENT: International Policy Statement for Universal Use of Kangaroo Mother Care for Preterm and Low Birthweight Infants. Retrieved from www.healthynewbornnetwork.org website: https://t.co/PxeMpfIJYw.

Anderson, J. M. (2000). Gender, 'Race', Poverty, Health and Discourses of Health Reform in the Context of Globalization: a Postcolonial Feminist Perspective in Policy Research. Nursing Inquiry, 7(4), 220-229.

Anderzen-Carlsson, A., Lamy, Z. C., Tingvall, M., Eriksson, R. M., \& Eriksson, M. (2014). Parental Experiences of Providing Skin-to-skin care to Their Newborn Infant - Part 2: A Qualitative Meta-synthesis. International Journal of Qualitative Studies on Health and Well-being, 9(24907). http://dx.doi.org/10.3402/qhw.v9.24907.

Anonymous. (1997). Science Wars and the need for respect and rigour. Nature, 385(6615), 373. doi: 10.1038/385373a0.

Ardal, F., Sulman, J., \& Fuller-Thomson, E. (2011). Support Like a Walking Stick: Parent-Buddy Matching for Language and Culture in the NICU. Neonatal Network 30(2), 89-98. doi: 10.1891/0730-0832.30.2.89.

Arner, J., \& Falmagne, R. J. (2007). Deconstructing Dualisms: The Both/And Conceptual Orientation and its Variant Linguistic Form. Feminism \& Psychology, 17(3), 357-371. doi:10.1177/0959353507079089.

Ash, J., \& Williams, M. E. (2016). Policies and Systems Support for Infant Mental Health in the Care of Fragile Infants and Their Families. Newborn \& Infant Nursing Reviews, 16, 316-321.

Ashby, B., \& Bromberg, S. R. (2016). Infant Mental Health with High Risk Populations. Newborn \& Infant Nursing Reviews, 16, 269-273. http://dx.doi.org/10.1053/j.nainr.2016.09.016. 
Atun-Einy, O., \& Scher, A. (2008). Measuring developmentally appropriate practice in neonatal intensive care units. Journal of Perinatology, 28, 218-225.

Auslander, G. K., Netzer, D., \& Arad, I. (2003). Parents' Satisfaction With Care in the Neonatal Intensive Care Unit: The Role of Sociocultural Factors.

Children's Health Care, 32(1), 17-36. doi: 10.1207/S15326888ChC3201_2.

Avenier, M.-J. (2010). Shaping a Constructivist View of Organizational Design Science. Organization Studies, 31(9-10), 1229-1255.

Ayers-Gould, J. N. (2000). Spirituality in birth: Creating sacred space within the medical model. International Journal of Childbirth Education, 15(1), 14-17.

Azulai, A., \& Rankin, J. A. (2012). Triangulation in Canadian Doctoral Dissertations on Aging. International Journal of Multiple Research Approaches, 6(2), 125140.

Badger, T. G. (2000). Action Research, Change and Methodological Rigour. Journal of Nursing Management, 8, 201-207.

Baillie, L. (2015). Promoting and Evaluating Scientific Rigour in Qualitative Research. Nursing Standard, 29(46), 36-42.

Baker, J. P. (2000). The Incubator and the Medical Discorvery of the Premature Infant. Journal of Perinatology, 5, 321-328.

Baley, J., \& COMMITTEE ON FETUS AND NEWBORN. (2015). Skin-to-Skin Care for Term and Preterm Infants in the Neonatal ICU. Pediatrics, 136(3), 596-599. doi:10.1542/peds.2015-2335.

Ballweg, D. D. (2001). Implementing Developmentally Supportive Family-Centered Care in the Newborn Intensive Care Unit as a Quality Improvement Initiative. Journal of Perinatal and Neonatal Nursing, 15(3), 58-73.

Barbera, R., A. (2008). Relationships and the Research Process: Participatory Action Research and Social Work. Journal of Progressive Human Services, 19(2), 140-159. doi:10.1080/10428230802475448.

Barbosa, V. M. (2013). Teamwork in the Neonatal Intensive Care Unit. Physical \& Occupational Therapy in Pediatrics, 33(1), 5-26. http://dx.doi.org/10.3109/01942638.2012.729556.

Barr, P. (2017). Compassion Fatigue and Compassion Satisfaction in Neonatal Intensive Care Unit Nurses: Relationships With Work Stress and Perceived Social Support. Traumatology, 23(2), 214-222. http://dx.doi.org/10.1037/trm0000115.

Barrere, C. C. (2007). Discourse Analysis of Nurse-Patient Communication in a Hospital Setting. Journal for Nurses in Staff Development, 23(3), 114-122.

Barton, P. (2018). The Elephant in the Room - Nursing and Māori Health Disparities. Kai Tiaki Nursing New Zealand, 24(4), 17-19.

Bastani, F., Rajai, N., Farsi, Z., \& Als, H. (2017). The Effects of Kangaroo Care on the Sleep and Wake States of Preterm Infants. The Journal of Nursing Research, 25(3), 231-239. doi:10.1097jnr.0000000000000194.

Bate, P., Mendel, P., \& Robert, G. (2008). Organizing for Quality: The improvement Journeys of Leading Hospitals in Europe and the United States. Oxford, United Kingdom: Radcliffe Publishing.

Bauer, M. (1996). THE NARRATIVE INTERVIEW: Comments on a Technique for Qualitative Data Collection. Papers in Social Research Methods. Qualitative Series no 1. Methodology Institute. London School of Economics and Political Science.

Baum, F., MacDougall, C., \& Smith, D. (2006). GLOSSARY: Participatory Action Research. Journal of Epidemilogy and Community Health, 60(10), 854-857. 
Baum, N., Weidberg, Z., Osher, Y., \& Kohelet, D. (2012). No Longer Pregnant, Not Yet a Mother: Giving Birth Prematurely to a Very-Low-Birth-Weight Baby. Qualitative Health Research, 22(5), 595-606. doi:10.1177/1049732311422899.

Baylis, R., Ewald, U., Gradin, M., Nyqvist, K., H., Rubertsson, C., \& Blomqvist, Y., T. (2014). First-Time Events Between Parents and Preterm Infants are Affected by the Designs and Routines of Neonatal Intensive Care Units. Acta Paediatrica, 103, 1045-1052.

Beck, C. T., \& Woynar, J. (2017). Posttraumatic Stress in Mothers While Their Preterm Infants Are in the Newborn Intensive Care Unit. A Mixed Research Syntheis. Advances in Nursing Science, 40(4). 337-355. doi: 10.1097/ANZ.0000000000000176.

Behruzi, R., Hatem, M., Goulet, L., \& Fraser, W. D. (2014). Perception of Humanization of Birth in a Highly Specialized Hospital: Let's Think Differently. Health Care for Women International, 35, 127-148. doi:10.1080/07399332.2013.857321.

Benoit, D. (2004). Infant-parent attachment: Definition, types, antecedents, measurement and outcome. Paediatr Child Health, 9(8), 541-545.

Benzies, K., M., Magill-Evans, J., E., Hayden, K., A., \& Ballantyne, M. (2013). Key Components of Early Intervention Programs for Preterm Infants and Their Parents: A Systematic Review and Meta-Analysis. BMC Pregnancy and Childbirth, 13(Suppl 1)(S10).

Berens, A. E., Jensen, S. K. G., \& Nelson III, C. A. (2017). Biological Embedding of Childhood Adversity: From Physiological Mechanisms to Clinical Implications. BMC Medicine, 15. doi:10.1186/s12916-017-0895-4.

Berger, R. (2015). Now I see it, now I don't: researcher's position and reflexivity in qualitative research. Qualitative Research, 15(2), 219-234. doi:10.1177/1468794112468475.

Bergh, A.-M., Arsalo, I., Malan, A. F., Patrick, M., Pattinson, R. C., \& Phillips, N. (2005). Measuring implementation progress in kangaroo mother care. Acta Paediatrica, 94, 1102-1108. doi:10.1080/08035250510028380.

Bergh, A.-M., Charpak, N., Ezeonodo, A., Udani, R. J., \& van Rooyen, E. (2012a). Education and training in the implememtation of kangaroo mother care. SAJCH, 6(2).

Bergh, A.-M., de Graft-Johnson, J., Khadka, N., Om'lniabohs, A., Udani, R. J., Pratomo, H., \& De Leon-Mendoza, S. (2016). The Three Waves in Implementation of Facility-Based Kangaroo Mother Care: A Multi-Country Case Study from Asia. BMC International Health and Human Rights, 16(4). doi:10.1186/s 12914-016-0080-4.

Bergh, A.-M., Kerber, K., Abwao, S., de-Graft Johnson, J., Aliganyira, P., Davy, K., Zoungrana, J. (2014). Implementing Facility-Based Kangaroo Mother Care Services: Lessons from a Multi-Country Study In Africa. BMC Health Services Research, 14, 1-10.

Bergh, A.-M., Manu, R., Davy, K., Van Rooyen, E., Asare, G. Q., Williams, J. K. A., Nang-beifubah, A. (2012b). Translating Research Findings into Practice The Implementation Of Kangaroo Mother Care in Ghana. Implementation Science, 7(75), 1-9.

Bergh, A.-M., \& Pattinson, R. C. (2003). Development of a Conceptual Tool for the Implementation of Kangaroo Mother Care. Acta Paediatr, 92, 709-714. doi:10.1080/08035250310002399. 
Bergh, A.-M., van Rooyen, E., \& Pattinson, R. C. (2008). Scaling Up Kangaroo Mother Care in South Africa: 'On-Site' Versus 'Off-Site Educational Facilitation. Human Resources for Health, 6(13). doi:10.1186/1478-4491-613.

Bergman, J., \& Bergman, N. (2013). Whose Choice? Advocating Birthing Practices According to Baby's Biological Needs. The Journal of Perinatal Education, 22(1), 8-13. http://dx.doi.org/10.1891/1058-1243.22.1.8.

Bergman, N. (2014). The Neuroscience of Birth - and the Case for Zero Separation. Curationis, 37(2). doi:10.4102/curationis.v37i2.1440.

Bergman, N. (2015). Neuroprotective Core Measures 1-7: Neuroprotection of Skinto-Skin Contact (SCC). Newborn \& Infant Nursing Reviews, 14, 142-146. doi:10.1053/j.nainr.2015.06.006.

Bergman, N. J., Linley, L. L., \& Fawcus, S. R. (2004). Randomized Controlled Trial of Skin-To-Skin Contact From Birth Versus Conventional Incubator for Physiological Stabilization in 1200- To 2199-Gram Newborns. Acta Paediatrica, 93, 779-785. doi:10.1080/08035250410028534.

Black, B. P., Holditch-Davis, D., \& Miles, M. S. (2009). Life Course Theory as a Framework to Examine Becoming a Mother of a Medically Fragile Preterm Infant. Research in Nursing \& Health, 32, 38-49. doi:10.1002/nur.20298.

Blackburn, A. C. (2009). Stories, Ethics and the Interpretation of Meaning: Bearing Witness to Mothers' Stories of their Neonatal Intensive Care Unit Experience. Doctor of Philosophy, The University of Southern Mississippi, Ann Arbor, Michigan, U.S.A. (3367164).

Bleakley, A. (2005). Stories as data, data as stories: making sense of narrative inquiry in clinical education. Medical Education, 39, 534-540. doi:10.1111/j.1365-2929.2005.02126.x

Bohnhorst, B. (2010). Skin to Skin Care in the Neonatal Intensive Care Unit: More Data Regarding Seriously Ill Infants are Badly Needed. Neonatology, 97, 318-320. doi:10.1159/000255164

Boundy, E., O., Dastjerdi, R., Spiegelman, D., Fawzi, W., W., Missmer, S., A., Lieberman, E., Chan, G., J. (2015). Kangaroo Mother Care and Neonatal Outcomes: A Meta-analysis. Pediatrics, 137(1).

Bourque, C. J., Dahan, S., Mantha, G., Robson, K., Reichherzer, M., \& Janvier, A. (2017). Improving Neonatal Care with the Help of Veteran Resource Parents: An Overview of Current Practices. Seminars in Fetal \& Neonatal Medicine, 1-8. https://doi.org/10.1016/j.siny.2017.10.005.

Bradby, H., Hargreaves, J., \& Robson, M. (2009). Story in Health and Social Care. Health Care Annal, 17, 331-344. doi:10.1007/s10728-009-0130-3

Braungart-Rieker, J. M., Zentall, S., Lickenbrock, D. M., Ekas, N. V., Oshio, T., \& Planalp, E. (2014). Attachment in the Making: Mother and Father Sensitivity and Infants' Responses During the Still-Face Paradigm. Journal of Experimental Child Psychology, 125, 63-84. http://dx.doi.org/10.1016/j.jecp.2014.02.007.

Brett, J., Staniszewska, S., Newburn, M., Jones, N., \& Taylor, L. (2011). A Systematic Mapping Review of Effective Interventions for Communicating With, Supporting and Providing Information to Parents of Preterm Infants. BMJ Open, 1. doi:10.1136/bmjopen-2010-000023.

Brockman, V. (2015). Implemeting the Mother-Baby Model of Nursing Care Using Models and Quality Improvement Tools. Nursing for Women's Health, 19(6), 490-503. https://doi.org/10.1111/1751-486X.12245. 
Broeder, J. L. (2003). Motherhood Too Soon: Beginning Mothering from the Neonatal Intensive Care Unit. Dissertation in partial fulfillment of the requirements for the degree of Doctor of Philosophy. Saint Louis University, St Louis, MO, United States.

Broom, M., Gardner, A., Kecskes, Z., \& Kildea, S. (2016). Transition From an Open-Plan to A Two-Cot Neonatal Intensive Care Unit: A Participatory Action Research Approach. Journal of Clinical Nursing, 26, 1939-1948. doi:10.1111/jocn.13509 1939.

Browne, A. J., Varcoe, C., Lavoie, J., Smye, V., Wong, S. T., Krause, M., Tu, D., Godwin, O., Khan, K., \& Fridkin, A. (2016). Enhancing Health Care Equity with Indigenous Populations: Evidence-based Strategies from an Ethnographic Study. BMC Health Services Research, 16(544). doi: 10.1186/s12913-016-1707-9.

Bryers-Brown, T. \& Trundle, C. (2017). Indigenizing Military Citizenship: Remaking State Responsibility and Care Towards Māori Veterans' Health Through the Treaty of Waitangi. AlterNative, 13(1), 43-50. doi: 10.1177/1177180117695410.

Buckley, S. J. (2015) Executive Summary of Hormonal Physiology of Childbearing: Evidence and Implications for Women, Babies and Maternity Care. The Journal of Perinatal Education, 24(3), 145-153.

Cahill, C., Cerecer, D. A. Q., \& Bradley, M. (2010). "Dreaming of...": Reflections on Participatory Action Research as a Feminist Praxis of Critical Hope. Journal of Women and Social Work, 25(4), 406-416. doi:10.1177/0886109910384576.

Camden, C., Swaine, B., Tetreault, S., \& Bergeron, S. (2009). SWOT Analysis of a Pediatric Rehabilitation Programme: A Participatory Evaluation Fostering Quality Improvement. Disability and rehabilitation, 31(16), 1373-1381. doi:10.1080/09638280802532696

Came, H. (2014). Sites of Institutional Racism in Public Health Policy Making in New Zealand. Social Science \& Medicine, 106. 214-220. http://dx.doi.org/10.1016/j.socscimed.2014.01.055.

Came, H., Cornes, R., \& McCreanor, T. (2016). Treaty of Waitangi in New Zealand Public Health Strategies and Plans 2006-2016. New Zealand Medical Journal, 131(1469), 32-37.

Came, H., \& Griffith, D. (2018). Tackling Racism as a "Wicked" Public Health Problem: Enabling Allies in Anti-Racism Praxis. Social Science \& Medicine 199, 181-188. http://dx.doi.org/10.1016/j.socscimed.2017.03.028.

Came, H., \& Humphries, M. (2014). Mopping up Institutional Racism: Activism on a Napkin. Journal of Corporate Citizenship, 54, 95-108.

Came, H., McCreanor, T., Doole, C., \& Rawson, E. The New Zealand Health Strategy 2016: Wither Health Equity? (2016). New Zealand Medical Journal, 129(1447), 72-77.

Came, H., McCreanor, T., \& Simpson, T. (2017). Health Activism Against Barriers to Indigenous Health in Aotearoa New Zealand. Critical Public Health, 27(4), 515-521. https://doi.org/10.1080/09581596.2016.1239816.

Came, H., \& Tudor, K. (2016). Bicultural Praxis: the Relevance of Te Tiriti o Waitangi to Health Promotion Internationally. International Journal of Health Promotion and Education, 54(4), 184-192. https://doi.org/10.1080/14635240.2016.1156009. 
Campbell-Yeo, M. L., Disher, T. C., Benoit, B. L., \& Johnston, C., C. (2015). Understanding Kangaroo Care and Its Benefits to Preterm Infants. Pediatric Health, Medicine and Therapeutics, 6, 15-32.

Cappella, E., Jackson, D. R., Bilal, C., Hamre, B. K., \& Soule, C. (2011). Bridging Mental Health and Education in Urban Elementary Schools: Participatory Research to Inform Intervention Development. School Psychology Review, 40(4), 486-508.

Carabine, J. (2001). Unmarried Motherhood 1830-1990: A genalogical analysis. In M. Wetherell, S. Taylor \& Yates (Eds.), Discourse as Data: A guide for Analysis. London: Sage publications and the Open University.

Carcary, M. (2009). The Research Audit Trial - Enhancing Trustworthiness in Qualitative Inquiry. The Electronic Journal of Business Research Methods, 7(1), 11-24.

Carnevale (1998). The Utility of Futility: the Construction of Bioethical Problems. Nursing Ethics 5(6), 509-517. https://doi.org/10.1177/096973309800500605.

Carroll, A. E. (2015). The Concern for Supply-Sensitive Neonatal Intensive Care Unit Care. If You Build Them, They Will Come. JAMA Pediatrics, 2015(169), 9. doi:10.1001/jamapediatrics.2015.1597.

Carter, S. M., \& Little, M. (2007). Justifying Knowledge, Justifying Method, Taking Action: Epistemologies, Methodologies, and Methods in Qualitative Research. Qualitative Health Research, 17(10), 1316-1328. doi:10.1177/1049732307306927.

Chan, G., Bergelson, I., Smith, E., R., Skotnes, T., \& Wall, S. (2017). Barriers and Enablers of Kangaroo Mother Care Implementation from a Health Systems Perspective: A Systematic Review. Health Policy and Planning, 32(10), 1466-1475. doi:10.1093/heapol/czx098.

Chan, G., J., Labar, A., S., Wall, S., \& Atun, R. (2016). Kangaroo Mother Care: a Systematic Review of Barriers and Enablers. Bulletin of the World Health Organization, 94, 130-141. http://dx.doi.org/10.2471/BLT.15.157818.

Chandra, Y., \& Shang, L. (2017). An RQDA-based Constructivist Methodology for Qualitative Research. Qualitative Market Research: An International Journal, 20(1), 90-112. https://doi.org/10.1108/QMR-02-2016-0014.

Chappell, P., Rule, P., \& Nkala, M. D. N. (2014). Troubling Power Dynamics: Youth with Disabilities as Co-Researchers in Sexuality Research in South Africa. Childhood, 21(3), 385-399.

Charpak, N., \& Ruiz, J. G. (2016). The Kangaroo Mother Care Method: from Scientific Evidence Generated in Colombia to Worldwide Practice. Journal of Clinical Epidemiology, 86, 125-128.

Charpak, N., Tessier, R., Ruiz, J. G., Hernandex, J. T., Uriza, F., Villegas, J., Maldonado, D. (2017). Twenty-year Follow-up of Kangaroo Mother Care Versus Traditional Care. Pediatrics, 139(1). doi:e20162063.

Cheyney, M. J. (2008). Homebirth as Systems-Challenging Praxis: Knowledge, Power, and Intimacy in the Birthplace. Qualitative Health Research, 18(2), 254-267. doi:10.1177/1049732307312393.

Chia, P., Sellick, K., \& Gan, S. (2005). The Attitudes and Practices of Neonatal Nurses in the use of Kangaroo Care. Australian Journal of Advanced Nursing, 23(4), 20-27.

Chiesa, M., \& Hobbs, S. (2008). Making sense of social research: How useful is the Hawthorne Effect? European jJournal of Social Psychology, 38, 67-74. doi:10.1002/ejsp.401. 
Cho, E.-S., Kim, S.-J., Kwon, M., S., Cho, H., Kim, E., H., Jun, E., M., \& Lee, S. (2016). The Effects of Kangaroo Care in the Neonatal Intensive Care Unit on the Physiological Functions of Preterm Infants, Maternal-Infant Attachment, and Maternal Stress. Journal of Pediatric Nursing, 31, 430-438.

Church, P., T., Luther, M., \& Asztalos, E. (2012). The Perfect Storm: The High Prevalence Low Severity Outcomes of the Preterm Survivors. Current Pediatric Reviews, 8, 142-151.

Clark, A. M. (2013). What are the Components of Complex Intervnetions in Healthcare? Theorizing Approaches to Parts, Powers and the Whole Intervention. Social Science \& Medicine, 93, 185-193. doi:10.1016/j.socscimed.2012.03.035.

Clark, S. (2017). Neonatal Intensive Care Unit - Student Nurses. In C. C. D. H. Board (Ed.), (pp. 1-24).

Cleveland, L., Hill, C. M., Pulse, W. S., DiCioccio, H. C., Field, T., \& White-Traut, R. (2017). Systematic Review of Skin-to-Skin Care for Full-Term, Healthy Newborns. JOGNN, 46, 857-869. https://doi.org/10.1016/j.jogn.2017.08.005.

Coley, R. L., Lynch, A. D., \& Kull, M. (2015). Early Exposure to Environmental Chaos and Children's Physical and Mental Health. Early Childhood Research Quarterly, 32, 94-104.

Conde-Agudelo, A., \& Diaz-Rossello, J. L. (2014). Kangaroo Mother Care to Reduce Morbidity and Mortality in Low Birthweight Infants (Review). Cochrane Database of Systematic Reviews(4). doi:10.1002/14651858.

Conde-Agudelo, A., \& Diaz-Rossello, J. L. (2016). Kangaroo mother care to reduce morbidity and mortality in low birth weight infants (Review). Cochrane Database of Systematic Reviews(8). doi:10.1002/14651858.CD002771.pub4.

Cooper, D. (1994). Productive, Relational and Everywhere? Conceptualising Power and Resistance within Foucauldian Feminism. Sociology, 28(2), 435-454.

Coughlin, M. (2011). Age-Appropriate Care of the Premature and Critically Ill Hospitalized Infant: Guideline for Practice. Retrieved from Glenview, IL.

Coughlin, M. (2013). Age-Appropriate Care of the Premature and Critically Ill Hospitalized Infant. Guideline for Practice. Retrieved from Glenview, IL, United States of America.

Coughlin, M. (2014). Transformative Nursing in the NICU: Trauma-informed Ageappropriate Care. New York, NY 10036: Springer Publishing Company.

Coughlin, M. (2017). Trauma-informed, Neuroprotective Care for Hopitalised Newborns and Infants. Infant, 13(5), 176-179.

Crowther, S., \& Hall, J. (2015). Spirituality and Spiritual Care in and Around Childbirth. Women and Birth, 28, 173-178. http://dx.doi.org/10.1016/j.wombi.2015.01.001.

Cruz, V., \& Walt, G. (2013). Brokering the Boundary Between Science and Advocacy: The Case of Intermittent Preventive Treatment Among Infants. Health Policy and Planning, 28(6), 616-625.

Curtis, E. (2013). Deserving of More: Framing of Māori Inequitites in Cardiovascular Care Remains a Challenge. The New Zealand Medical Journal, 126(1379). http://journal.nzma.org.nz/journal/126-1379/5756/.

D'Agata, A. L., Coughlin, M., \& Sanders, M. R. (2018). Clinician Perceptions of the NICU Infant Experience: Is the NICU Hospitalization Traumatic? Journal Article. College of Nursing. Rhode Island University. 
D'Agata, A. L., Sanders, M. R., Grasso, D. J., Young, E. E., Cong, X., \& McGrath, M. J. (2017). Unpacking the Burden of Care For Infants In The NICU. Infant Mental Health Journal, 38(2), 306-317. doi:10.1002/imhj.21636.

D'Agata, A. L., Young, E. E., Cong, X., Grasso, D. J., \& McGrath, J. M. (2016). Infant Medical Trauma in the Neonatal Intensive Care Unit (IMTN): A Proposed Concept for Science and Practice. Advances in Neonatal Care, 16(4), 289-297. doi:10.1097/ANC.0000000000000309

da Silva, L. J., da Silva, L. R., \& Christoffel, M. M. (2009). Technology and Humanization of the Neonatal Intensive Care Unit: Reflections in the Context of the Health-illness Process. Rev Esc Enferm USP, 43(3), 678-682.

Daramas, T., Chontawan, R., Yenbut, J., Wittayasooporn, J., \& Nantachaipan, P. (2008). Enhancing Nursing Practice in Developmental Care for Preterm Infants. Thai Journal of Nursing Research, 12(2), 83-94.

Davanzo, R., Brovedani, P., Travan, L., Kennedy, J., Crocetta, A., Sanesi, C., De Cunto, A. (2013). Intermittent Kangaroo Mother Care: A NICU Protocol. Journal of Human Lactation, 29, 332-338. doi:10.1177/0890334413489375.

Davies, D., \& Dodd, J. (2002). Qualitative Research and the Question of Rigor. Qualitative Health Research, 12, 279-289. doi:10.1177/104973230201200211.

Davis-Floyd, R. E. (2001). The Technocratic, Humanistic, and Holistic Paradigms of Childbirth. International Journal of Gynecology \& Obstetrics, 75, S5-S23.

Davis-Floyd, R. E., Pascali-Bonaro, D., Leslie, M. S., \& de Leon, R. G. P. (2011). The International MotherBaby Childbirth Initiative: Working to Create Optimal Maternity Care Worldwide. International Journal of Childbirth, 1(3), 196-212. http://dx.doi.org/10.1891/2156-5287.1.3.196.

Davis, J. E. (2016). Biomedicine and Its Cultural Authority. The New Atlantis, 48, 60-77.

Davis, L., Mohay, H., \& Edwards, H. (2003). Mothers' Involvement in Caring for their Premature Infants: An Historical Overview. Journal of Advanced Nursing, 42(6), 578-586.

Denison, H. J., Eng, A., Barnes, L. A., Cheng, S., Mannetje, A., Haddock, K., Douwes, J., Pearce, N., Ellison-Loschmann, L. (2018). Inequities in Exposure to Occupational Risk Factors Between Māori and non-Māori workers in Aotearoa New Zealand. Epidemiology of Community Health, 72, 809-816. doi: 10.1136/jech-2018-210438.

De Oliveira, B. (2018). On the News Today: Challenging Homelessness Through Participatory Action Research. Housing, Care and Support, 21(1), 13-25.

Del Fabbro, A., \& Cain, K. (2016). Infant Mental Health and Family Mental Health Issues. Newborn \& Infant Nursing Reviews, 16, 281-284.

DiMenna, L. (2006). Considerations for Implementation of a Neonatal Kangaroo Care Protocol. Neonatal Network, 25(6), 405-412.

Dogherty, E. J., Harrison, M., B., Graham, I., D., Vandyk, A., D., \& Keeping-Burke, L. (2013). Turning Knowledge Into Action at the Point-of-Care: The Collective Experience of Nurses Facilitating the Implementation of Evidence-Based Practice. Worldviews on Evidence-Based Nursing, 10(3), 129-139.

Douche, J. R. (2007). Caesarean Section in the Absence of Clinical Indications: Discourses Constituting Choice in Childbirth. Doctor of Philosophy in Midwifery, Massey University, Palmerston North, New Zealand. 
Downe, S. (2008). Metasynthesis: a guide to knitting smoke. Evidence Based Midwifery, 6(1), 4-8.

Edley, N. (2001). Analysing Masculinity: Interpretative Repertoires, Ideological Dilemmas and Subject Positions. In M. Wetherell, S. Taylor \& Yates (Eds.), Discourse as data: A guide for analysis. London: Sage publications and the Open University.

Emery-Whittington, I., Te Maro, B. (2018). Decolonising Occupation: Causing Social Change to Help Our Ancestors Rest and Our Descendants Thrive. New Zealand Journal of Occupational Therapy, 65(1), 12-19.

Engler, A., J., Ludington-Hoe, S., M., Cusson, R., M., Adams, R., Bhansen, M., Brumbaugh, E., Williams, D. (2002). Kangaroo Care: National Survey of Practice, Knowledge, Barriers, and Perceptions. American Journal of Maternal Child Nursing, 27(3), 146-153.

Engmann, C., Wall, S., Darmstadt, G., Valsangkar, B., \& Claeson, M. (2013). Consensus on Kangaroo Mother Care Acceleration. www.thelancet.com, 382(November), e26-e27.

Erdei, C., \& Dammann, O. (2014). THE PERFECT STORM: Preterm Birth, Neurodevelopmental Mechanisms, and Autism Causation. Perspectives in Biology and Medicine, 47(4), 470-481.

Ericson-Lidman, E., \& Strandberg, G. (2018). Using a Developed Participatory Action Research Process in Practice to Help Care Providers Deal with Troubled Conscience in Residential Care Of Older People. Action Research, 16(2), 190-206 doi:10.1177/1476750316678916.

Evans, C. A., \& Porter, C. L. (2009). The Emergence of Mother-Infant CoRegulation During The First Year: Links to Infants' Developmental Status and Attachment. Infant Behavior \& Development, 32, 147-158. doi:10.1016/j.infbeh.2008.12.005.

Evans, S. D., Hanlin, C. E., \& Prilleltensky, I. (2007). Blending Ameliorative and Transformative Approaches in Human Service Organizations: A Case Study. Journal of Community Psychology, 35(3), 329-346. doi:10.1002/jcop. 20151.

Evans, T., Whittingham, K., \& Boyd, R. (2012). What Helps the Mother of a Preterm Infant Become Securely Attached, Responsive and Well-Adjusted? Infant Behavior \& Development, 35, 1-11. doi:0.1016/j.infbeh.2011.10.002.

Evereklian, M., \& Posmontier, B. (2017). The Impact of Kangaroo Care on Premature Infant Weight Gain. Journal of Pediatric Nursing, 34, e10-e16. http://dx.doi.org/10.1016/j.pedn.2017.02.006.

Farry, A., \& Crowther, S. (2014). Cultural Safety in New Zealand Midwifery Practice: Part 1. The Practising Midwife, June, 10-13.

Feeley, N., Genest, C., Niela-Vilen, H., Charbonneau, L., \& Axelin, A. (2016). Parents and Nurses Balancing Parent-Infant Closeness and Separation: A Qualitative Study of NICU Nurses' Perceptions. BMC Pediatrics, 16(1). http://dx.doi.org/10.1186/s12887-016-0663-1.

Fegran, L., Helseth, S., \& Slettebo, A. (2006). Nurses as Moral Practitioners Encountering Parents in Neonatal Intensive Care Units. Nursing Ethics, 13(1), 52-64. doi: 10.1191/0969733006ne849oa.

Feldman, R. (2004). Mother-Infant Skin-to-Skin Contact (Kangaroo Care): Theoretical, Clinical, and Empirical Aspects. Infants and Young Children, 17(2), 145-161.

Feldman, R. (2007). Maternal-Infant Contact and Child Development: Insights from the Kangaroo Intervention. In: L. L'Abate (Ed.), Low-Cost Approaches to 
Promote Physical and Mental Health. Springer. New York, NY. https://doi.org/10.1007/0-387-36899-X_16.

Feldman, R. (2015a). Mutual influences between child emotion regulation and parent-child reciprocity support development across the first 10 years of life: Implications for developmental psychopathology. Development and Psychopathology, 27, 1007-1023. doi:10.1017/S0954579415000656.

Feldman, R. (2015b). The neurobiology of mammalian parenting and the biosocial context of human caregiving. Hormones and Behavior. doi:10.1016/j.yhbeh.2015.10.001.

Feldman, R. (2015c). Sensitive periods in human social development: New insights from research on oxytocin, synchrony, and high-risk parenting. Development and Psychopathology, 27, 369-395. doi:10.1017/S0954579415000048.

Feldman, R., \& Eidelman, A. I. (2007). Maternal Postpartum Behavior and the Emergence of Infant-Mother and Infant-Father Synchrony in Preterm and Full-Term Infants: The Role of Neonatal Vagal Tone. Developmental Psychobiology, 49(3), 290-302. doi:10.1002/dev.

Feldman, R., Rosenthal, Z., \& Eidelman, A. I. (2014). Maternal-Preterm Skin-toSkin Contact Enhances Child Physiologic Organization and cognitive Control Across the First 10 Years of Life. Biol Psychiatry, 75, 56-64.

Feldman, R., Weller, A., Sirota, L., \& Eidelman, A. I. (2002). Skin-to-Skin Contact (Kangaroo Care) Promotes Self-Regulation in Premature Infants: SleepWake Cyclicity, Arousal Modulation, and Sustained Exploration. Developmental Psychology, 38(2), 194-207. doi:10.1037//00121649.38.2.194.

Fenwick, J., Barclay, L., \& Schmied, V. (2001). Struggling to Mother: A Consequence of Inhibitive Nursing Interactions in the Neonatal Nursery. Journal of Perinatal and Neonatal Nursing, 49-64.

Ferreyra, C. (2006). Practicality, Positionality, and Emancipation: Reflections on Participatory Action Research with a Watershed Partnership. Systemic Practice and Action Research, 19, 577-598. doi:10.1007/s11213-006-9044-2.

Feucht, U., Van Rooyen, E., Skhosana, R., \& Bergh, A.-M. (2016). Taking Kangaroo Mother Care Forward in South Africa: The Role of District Clinical Specialist Teams. South African Medical Journal, 106(1), 49-52.

Fialho, F. A., Vargas, I. M., \& Santos, R. S. (2016). Humanization Permeating Newborn Nursing Care. Journal of Nursing UFPE on line, 10(7), 2412-2419. doi:10.5205/reuol.9106-80230-1-SM1007201615.

Finlayson, K., Dixon, A., Smith, C., Dykes, F. \& Flacking, R. (2014). Mothers' Perceptions of Famiy Centred Care in Neonatal Intensive Care Units. Sexual \& Reproductive Healthcare 5, 119-124. http://dx.doi.org/10.1016/j.srhc.2014.06.003.

Flacking, R., \& Dykes, F. (2013). 'Being in a Womb' or 'Playing Musical Chairs': the Impact of Place and Space on Infant Feeding in NICUs. BMC Pregnancy and Childbirth, 13(179).

Flacking, R., Ewald, U., Nyqvist, K. H., Starrin, B. (2006). Trustful Bonds: A Key to "Becoming a Mother" and to Reciprocal Breastfeeding. Stories of Mothers of Very Preterm Infants at a Neonatal Unit. Social Science \& Medicine, 62, 7080. doi: 10.1016/j.socscimed.2005.05.026.

Flacking, R., Lehtonen, L., Thomson, G., Axelin, A., Ahlqvist, S., Moran, V. H., Ewald, U., Dykes, F., the SCENE group (2012). Closeness and Separation in Neonatal Intensive Care. Acta Paediatrica, 101, 1032-1037. 
Flacking, R., Thomson, G., \& Axelin, A. (2016). Pathways to Emotional Closeness in Neonatal Units - A Cross-National Qualitative Study. BMC Pregnancy and Childbirth, 16(170). doi:10.1186/s12884-016-0955-3.

Fleck, P. (2015). Mother's Lived Experience During Repair of Long-gap Esophageal Atresia: A Phenomenological Inquiry. Dissertation in partial fulfillment of the requirements for the degree of Doctor of Philosophy (Nursing). Northeastern University, Boston, Massachusetts.

Fleck, P. (2016). Connecting Mothers and Infants in the Neonatal Intensive Care Unit. Newborn \& Infant Nursing Reviews, 16, 92-96.

Fletcher, A. J., \& Marchildon, G. P. (2014). Using the Delphi Method for Qualitative, Participatory Action Research in Health Leadership. International Journal Of Qualtitative Methods, 13, 8 - 18.

Foster, V., \& Young, A. (2015). Reflecting on Participatory Methodologies: Research with Parents of Babies Requiring Neonatal Care. International Journal of Social Research Methodology, 18(1), 91-104. doi:10.1080/13645579.2013.853964.

Foucault, M. (1972). The Archaeology of Knowledge. London: Tavistock Publications Ltd.

Foucault, M. (1973). The Birth of the Clinic: An Archaeology of Medical Perception. London: Tavistock Publications Ltd.

Foucault, M. (1977 [1979]). Discipline and Punish: The Birth of the Prison (A. Sheridan, Trans.) New York: Random House, Inc.

Foucault, M. (1978 [2008]). The Will to Knowledge. The History of Sexuality. An Introduction. Volume 1 (R. Hurley, Trans.) New York: Vantage Books, Random House, Inc.

Fox, S. E., Levitt, P., \& Nelson III, C. A. (2010). How the Timing and Quality of Early Experiences Influence the Development of Brain Architecture. Child Development, 81(1), 28-40.

Foxall, D. (2013). Barriers in Education of Indigenous Nursing Students: A Literature Review. Nursing Praxis in New Zealand, 29(3), 33-39.

Franklin, C. (2006). The Neonatal Nurse's Role in Parental Attachment in the NICU. Critical Care Nursing Quarterly, 29(1), 81-85.

Frey, H. A., \& Klebanoff, M. A. (2016). The Epidemiology, Etiology, and Costs of Preterm Birth. Seminars in Fetal \& Neonatal Medicine, 21, 68-73. http://dx.doi.org/10.1016/j.siny.2015.12.011.

Friesen-Storms, J., H, H, M., Moser, A., vander Loo, S., Beurskens, J., H, M., \& Bours, G., J, J, W. (2014). Systematic Implementation of Evidence-Based Practice in a Clinical Nursing Setting: A Participatory Action Research Project. Journal of Clinical Nursing, 24, 57-68. doi:10.1111/jocn.12697.

Furman, L. (2016). Kangaroo Mother Care 20 Years Later: Connecting Infants and Families. Pediatrics, 139(1). doi:10.1542/peds.2016-3332.

Gao, H., Guihua, H., Honglian, G., Dong, R., Fu, H., Wang, D., . . Zhang, H. (2015). Effect of Repeated Kangaroo Mother Care on Repeated Procedural Pain in Preterm Infants: A Randomized Controlled Trial. International Journal of Nursing Studies, 52, 1157-1165.

Garcia, E. R., \& Yim, I. S. (2017). A Systematic Review of Concepts Related to Women's Empowerment in the Perinatal Period and their Associations with Perinatal Depressive Symptoms and Premature Birth. BMC Pregnancy and Childbirth, 17 (Suppl 2)(347), 58-69. doi:10.1186/s12884-017-1495-1. 
Gartner, L. M., \& Gartner, C. B. (1992). The Care of Premature Infants: Historical Perspective. Paper presented at the NEONATAL INTENSIVE CARE: A HISTORY OF EXCELLENCE, Bethesda, Maryland.

Gatenby, B., \& Humphries, M. (2000). Feminist Participatory Action Research: Methodological and Ethical Issues. Women's Studies International Forum, 23(1), 89-105.

Gibbins, S., Hoath, S., B., Coughlin, M., Gibbins, A., \& Franck, L. (2009). The Universe of Developmental Care: A New Conceptual Model for Application in the Neonatal Intensive Care Unit. Advances in Neonatal Care, 8(3), 141147.

Gluck, L. (1992). Conceptualization and Initiation of a Neonatal Intensive Care Nursery in 1960. Paper presented at the NEONATAL INTENSIVE CARE: A HISTORY OF EXCELLENCE, Bethesda, Maryland.

Golden, J. (2017). "Doctor's Don't Do So Much Good": Traditional Practices, Biomedicine, and Infant Care in the 20th-Century United States. Nursing History Review, 25, 86-102. http://dx.doi.org/10.1891/1062-8061.25.86.

Goldenberg, R. I., Culhane, J. F., Iams, J. D., \& Romero, R. (2008). Epidemiology and causes of preterm birth. Lancet, 371, 75-84.

Goldstein Ferber, S., \& Makhoul, I. R. (2004). The Effect of Skin-to-Skin Contact (Kangaroo Care) Shortly After Birth on the Neurobehavioral Responses of the Term Newborn: A Randomized, Controlled Trial. Pediatrics, 113(4), 858-865.

Gondwe, K. W., \& Holditch-Davis, D. (2015). Posttraumatic Stress Symptoms in Mothers of Preterm Infants. International Journal of Africa Nursing Sciences, 3, 8-17. http://dx.doi.org/10.1016/j.ijans.2015.05.002.

Gooding, J. S., Cooper, L. G., Franck, L. S., Howse, J. L., \& Berns, S. D. (2011). Family Support and Family-Centred Care in the Neonatal Intensive Care Unit: Origins, Advances, Impact. Seminars in Perinatology, 35, 20-28. doi: 10.1053/j.semperi.2010.10.004.

Goodyear-Smith, F. (2017). Collective Enquiry And Reflective Action in Research: Towards a Clarification of the Terminology. Family Practice, 34(3), 268271. doi:10.1093/fampra/cmw098.

Gorli, M., Nicolini, D., \& Scaratti, G. (2015). Reflexivity in Practice: Tools and Conditions for Developing Organizational Authorship. Human Relations, 68(8), 1347-1375. doi:10.1177/0018726714556156.

Greenhalgh, T., Snow, R., Ryan, S., Rees, S., \& Salisbury, H. (2015). Six 'Biases' Against Patients and Carers in Evidence-Based Medicine. BMC Medicine, 13(200). doi:10.1186/s12916-015-0437-x.

Grigg, C. P., \& Tracy, S. K. (2013). New Zealand's Unique Maternity System. Women and Birth, 26, e59-e60. http://dx.doi.org/10.1016/j.wombi.2012.09.006

Griscti, O., Aston, M., Warner, G., Martin-Misener, R., \& McLeod, D. (2017). Power and Resistance Within the Hospital's Hierarchical System: The Experiences of Chronically Ill Patients. Journal of Clinical Nursing, 26, 238247. doi:10.1111/jocn.13382.

Grol, R., \& Wensing, M. (2004). What drives change? Barriers To and Incentives for Achieving Evidence-Based Practice. Medical Journal of Australia, 180(S57S60). 
Grunau, R. E. (2013). Neonatal Pain in Very Preterm Infants: Long-Term Effects on Brain, Neurodevelopment and Pain Reactivity. Rambam Maimonides Medical Journal, 4(4), e0025. doi:10.5041/RMMJ.10132

Guenther, T., Moxon, S., Valsangkar, B., Wetzel, G., Ruiz, J. G., Kerber, K., Mazia, G. (2017). Consensus-Based Approach to Develop a Measurement Framework and Identify a Core Set of Indicators to Track Implementation and Progress Towards Effective Coverage of Facility-Based Kangaroo Mother Care. Journal of Global Health, 7(2), 395-406. doi:10.7189/jogh.07.020801.

Gulla, K., Dahlo, R., \& Eilertsen, M.-E., B. (2017). From the Delivery Room to the Neonatal Intensive Care Unit - Mothers' Experiences with Follow-Up of Skin-To-Skin Contact after Premature Birth. Journal of Neonatal Nursing, 23, 253-257.

Gunnar, M. R., \& Quevedo, K. M. (2008). Early Care Experiences and HPA Axis Regulation in Children: A Machanism for Later Trauma Vulnerability. Progress in Brain Research, 167, 137-149. doi:10.1016/S00796123(07)67010-1.

Gustafson, D. L., \& Brunger, F. (2014). Ethics, "Vulnerability," and Feminist Participatory Action Research With a Disability Community. Qualitative Health Research, 24(7), 997-1005. doi:10.1177/1049732314538122.

Hall, S. L., Phillips, R., \& Hynan, M. T. (2016). Transforming NICU Care to Provide Comprehensive Family Support. Newborn \& Infant Nursing Reviews 16, 69-73.

Hall, S. L., Hynan, M. T., Phillips, R., Lassen, S., Craig, J. W., Goyer, E., Cohen, H. (2017). The Neonatal Intensive Parenting Unit: an Introduction. Journal of Perinatology, 1-6. doi:10.1038/jp.2017.108. http://dx.doi.org/10.1053/j.nainr.2016.03.008.

Harris, R. B., Stanley, J., Cormack, D. M. (2018). Racism and Health in New Zealand: Prevalence Over Time and Associations Between Recent Experience of Racism and Health and Wellbeing Measures using National Survey Data. PLoS ONE, 13(5). https://doi.org/10.1371/journal.pone.0196476.

Harris, R., Tobias, M., Jeffreys, M., Waldegrave, K., Karlsen, S., \& Nazroo, J. (2006). Racism and Health: The Relationship Between Experience of Racial Discrimination and Health in New Zealand. Social Science \& Medicine, 64, 1428-1441.

Harrison, M. S., \& Goldenberg, R. L. (2016). Global burden of prematurity. Seminars in Fetal \& Neonatal Medicine, 21(2), 74-79. https://doi.org/10.1016/j.siny.2015.12.007.

Harrison, W., \& Goodman, D. (2015). Epidemiologic Trends in Neonatal Intensive Care, 2007-2012. JAMA Pediatrics, 169(9), 855-862. doi:10.1001/jamapediatrics.2015.1305.

Heermann, J. A., Wilson, M. E., \& Wilhelm, P. A. (2005). Mothers in the NICU: Outsider to Partner. Pediatric Nursing, 31(3), 176-181.

Helin, I. (2015). Mother-infant Relationships in the NICU: A Multiple Case Study Approach. Doctor of Philosophy, Program in Child Development, Chicago, Illinois, United States.

Hendricks-Munoz, K. D., Louie, M., Li, Y., Chhun, N., Prendergast, E. D., \& Ankola, P. (2010). Factors That Influence Neonatal Nursing Perceptions of 
Family-Centered Care and Developmental Care Practices. American Journal of Perinatology, 27, 193-200. http://dx.doi.org/10.1055/s-0029-1234039.

Hernandez, N., L., Rubio-Grillo, M., H., \& Lovera, A. (2016). Strategies for Neonatal Developmental Care and Family-Centered Neonatal Care. Investigacion y Educacion en Enfermeria, 34(1), 104-112. doi:10.17533/udea.iee.v34n1a12.

Hertzman, C. (2012). Putting the Concept of Biological Embedding in Historical Perspective. Proceedings of the National Academy of Sciences of the United States of America, 109(Supplement 2), 17160-17167.

Higgins, I., \& Dullow, A. (2003). Parental Perceptions of Having a Baby in a Neonatal Intensive Care Unit. Neonatal, Paediatric and Child Health Nursing, 6(3), 15-20.

Hill, S., Sarfati, D., Blakely, T., Robson, B., Purdie, G., Chen, J., Dennett, E., Cormack, D., Cunningham, R., Dew, K., McCreanor, T., Kawachi, I. (2010). Survival Disparitites in Indigenous and non-Indigenous New Zealanders with Colon Cancer: the Role of Patient Comorbidity, Treatment and Health Service Factors. Journal of Epidemiology \& Community Health, 64, 117-123. doi: 10.1136/jech.2008.083816.

Himuro, N., Miyagishima, S., Kozuka, N. H. T., \& Mori, M. (2015). Measurement of Family-Centered Care in the Neonatal Intensive Care Unit and Professional Background. Journal of Perinatology, 35, 284-289. doi:10.1038/jp.2014.204.

Hofer, M. A. (2006). Psychobiological Roots of Early Attachment. Current Directions in Psychological Science, 15(2), 84-88.

Holditch-Davis, D., Scher, M., Schwartz, T., \& Hudson-Barr, D. (2004). Sleeping and Waking State Development in Preterm Infants. Early Human Development, 80, 43-64. doi:10.1016/j.earlhumdev.2004.05.006.

Holmes, M. (2007). What is Gender? Sociological Approaches. London, England: Sage.

Hossain, S., Shah, P. S., Ye, X. Y., Darlow, B. A., Lee, S. K., \& Lui, K. (2015). Outcome Comparison of Very Preterm Infants Cared for in the Neonatal Intensive Care Units in Australia and New Zealand and in Canada. Journal of Paediatrics and Child Health, 51, 881-888.

Hubbard, J. M., \& Gattman, K. R. (2017). Parent-Infant Skin-to-Skin Contact Following Birth: History, Benefits, and Challenges. Neonatal Network, 36(2), 89-97.

Huebner, G., Boothby, N., Aber, J. L., Darmstadt, G. L., Diaz, A., Masten, A. S., Zeanah, C. H. (2016). Beyond Survival: The Case for Investing in Young Children Globally. Retrieved from Washington, DC.: https://nam.edu/wpcontent/uploads/2016/09/Beyond-Survival-The-Case-for-Investing-inYoung-Children-Globally.pdf.

Human Rights Commission. (2012). A Fair Go For All? Rite Tahi Tātou Katoa? Addressing Structural Discrimination in Public Services. Retrieved from https://www.hrc.co.nz/files/2914/2409/4608/HRC-StructuralReport_final_webV1.pdf.

Huria, T., Palmer, S., Beckert, L., Williman, J., Pitama, S. (2018). Inequity in Dialysis Related Practices and Outcomes in Aotearoa/New Zealand: a Kaupapa Māori analysis. International Journal for Equity in Health, 17(27). https://doi.org/10.1186/12939-018-0737-9. 
Hurst, I. (2001a). Mothers' Strategies to Meet Their Needs in the Newborn Intesive Care Nursery. Journal of Perinatal and Neonatal Nursing, 15(2), 65-82.

Hurst, I. (2001b). Vigilant Watching Over: Mothers' Actions to Safeguard Their Premature Babies in the Newborn Intensive Care Nursery. Journal of Perinatal and Neonatal Nursing, 15(3). 39-57.

Hyslop, I., \& Keddell, E. (2018). Outing the Elephants: Exploring a New Paradigm for Child Protection Social Work. Social Sciences, 7(105). doi: 10.3390/socsci7070105.

Ichijima, E., Kirk, R., \& Hornblow. (2011). Parental Support in Neonatal Intensive Care Units: A Cross-Cultural Comparison Between New Zeland and Japan. Journal of Pediatric Nursing, 26, 206-215. doi: 10.1016/j.pedn.2009.10.003.

Jaafar, S. H., Ho, J. J., \& Lee, K.S. (2016). Rooming-in for New Mother and Infant Versus Separate Care for Increasing the Duration of Breastfeeding. Cochrane Database of Systematic Reviews(8). doi:10.1002/14651858.CD006641.pub3.

Jacobs, G. (2010). Conflicting Demands and the Power of Defensive Routines in Participatory Action Research. Action Research, 8(4), 367-386. doi:10.1177/1476750310366041.

Jacobs, S., E., Sokol, J., \& Ohlsson, A. (2002). The Newborn Individualized Developmental Care and Assessment Program is not Supported by Metaanalyses of the Data. The Journal of Pediatrics 140(6), 699-706.

Jehonathan, B., Cormack, D., Harris, R., Paradies, Y. (2017). Racism and Health Service Utilisation: A Systematic Review and Meta-Analysis. PLoS ONE 12(12). https://doi.org/10.1371/journal.pone.0189900.

Jenkins, K. (2015). Feminist Methodologies: Unsettling Multiple Boundaries in Development in Negotiating Boundaries and Borders, 83-103. https://doi.org/10.1016/S1042-3192(06)08005-0.

Johnson, K. (2013). Maternal-Infant Bonding: A Review of the Literature. International Journal of Childbirth Education, 28(3), 17-22.

Johnson, S. L. (2015). Workplace Bullying Prevention: A Critical Discourse Analysis. Journal of Advanced Nursing, 71(10), 2384 - 2392. doi:10.1111/jan.12694.

Johnstone, M-J., \& Kanitsaki, O. (2010). The Neglect of Racism as an Ethical Issue in Health Care. Journal of Immigrant Minority Health, 12. 489-495. doi: 10.1007/s10903-008-9210-y.

Jordan, R. G., \& Murphy, P. A. (2009). Risk Assessment and Risk Distortion: Finding the Balance. Journal of Midwifery \& Women's Health, 54, 191-200. doi:10.1016/j.jmwh.2009.02.001.

Kadetz, P. (2014). Colonizing Safety: Creating Risk through the Enforcement of Biomedical Constructions of Safety. East Asian Science, Technology and Society: An International Journal, 8, 81-106. doi:10.1215/187521602405624.

Kadivar, M., Seyedfatemi, N., Akbari, N., \& Haghani, H. (2017). The effect of narrative writing of mothers on their statisfaction in the neonatal intensive care unit. Journal of Maternal-Fetal Neonatal Medicine, 30(3), 352-356. doi:10.3109/14767058.2016.1173028.

Kallan, J. C. (2013). "IT'S LIKE A DIFFERENT KIND OF PARENTING": CONSTRUCTIONS OF GOOD AND BAD PARENTING IN NEONATAL INTENSIVE CARE. Doctor of Philosophy Dissertation, Temple University, Ann Arbor, MI 48106. 
Karatzias, T., Chouliara, Z., Maxton, F., Freer, Y., \& Power, K. (2007). Posttraumatic Symptomatology in Parents with Premature Infants: A Systematic Review of the Literature. Journal of Prenatal \& Perinatal Psychology \& Health, 21(3), 249-260.

Karl, D., J., \& O'Hare, C. M. (2006). Reconceptualizing the Nurse's Role in the Newborn Period as an "ATTACHER". American Journal of Maternal/Child Nursing, 31(4), 257-262.

Kearns, R., Moewaka-Barnes, H., McCreanor, T. (2009). Placing Racism in Public Health: a Perspective from Aotearoa/New Zealand. GeoJournal, 74, 123-129. doi: 10.1007/s10708-009-9261-1.

Kearvell, H., \& Grant, J. (2010). Getting Connected: How Nurses Can Support Mother/Infant Attachment in the Neonatal Intensive Care Unit. Australian Journal of Advanced Nursing, 27(3), 75-82.

Kellett, P., Gregory, D. M., \& Evans, J. (2014). Patriarchal Paradox: Gender Performance and Men's Nursing Careers. Gender in Management: An International Journal, 29(2), 77-90. https://doi.org/10.1108/GM-06-20130063.

Kelly, P., J. (2005). Practical Suggestions for Community Interventions Using Participatory Action Research. Public Health Nursing 22(2), 65-73.

Kemmis, S. (2009). Action Research as a Practice-based Practice. Educational Action Research, 17(3), 463-474. doi:10.1080/09650790903093284.

Kenney, C. (2011). Midwives, Women and Their Families: A Maori Gaze: Towards Partnerships for Maternity Care in Aotearoa New Zealand. AlterNative (Nga Pae o te Maramatanga), 7(2), 123-137.

Khanlou, N., \& Peter, E. (2005). Participatory Action Research: Considerations For Ethical Review. Social Science \& Medicine, 60, 2333-2340. doi:10.1016/j.socscimed.2004.10.004.

Kuhn, P., Sizun, J., \& Casper, C. (2018). Recommendations on the Environment for Hospitalised Newborn Infants from the French Neonatal Society: Rationale, Methods and First Recommendation on Neonatal Intensive Care Unit Design. Acta Paediatrica, 107, 1850-1856. doi: 10.1111/apa.14501.

Khungern, J., Krairiksh, M., Taasaniyom, N., \& Sritanyarat, W. (2006). Hospital Quality Improvement: A Case Study of a General Hospital under the Ministry of Public Health. Thai J Nurs Res, 10(3), 191-200.

Kidd, S. A., \& Kral, M. J. (2005). Practicing Participatory Action Research. Journal of Counseling Psychology, 52(2), 187-195. doi:10.1037/0022-0167.52.2.187.

Kiechl-Kohlendorfer, U., Merkle, U., Deufert, D., Neubauer, V., Peglow, U. P., \& Griesmaier, E. (2015). Effect of Developmental Care For Very Premature Infants on Neurodevelopmental Outcome At 2 Years Of Age. Infant Behavior \& Development, 39, 166-172.

Kim, P. (2016). The Maternal Brain and Its Plasticity in Humans. Hormones and Behavior, 77, 113-123.

Kindon, S., \& Elwood, S. (2009). Introduction: More than Methods - Reflections on Participatory Action Research in Geographic Teaching, Learning and Research. Journal of Geography in Higher Education, 33(1), 19-32. doi:10.1080/03098260802276474.

Klocker, N. (2012). Doing Participatory Action Research and Doing a PhD: Words of Encouragement for Prospective Students. Journal of Geography in Higher Education, 36(1), 149-163. http://dx.doi.org/10.1080/03098265.2011.589828 
Klocker, N. (2015). Participatory Action Research: The Distress Of (Not) Making a Difference. Emotion, Space and Society, 17, 37-44. http://dx.doi.org/10.1016/j.emospa.2015.06.006.

Koch, T., \& Kralik, D. (2006). Participatory Action Research in Healthcare. 9600 Garsington Road, Oxford OX4 2DQ, UK: Blackwell Publishing Ltd.

Koch, T., Selim, P., \& Kralik, D. (2002). Enhancing Lives Through the Development of a Community-Based Participatory Action Research Programme. Journal of Clinical Nursing, 11, 109-117.

Kologeski, T. K., Strapasson, M. R., Schneider, V., \& Renosto, J. M. (2017). Skin to Skin Contact of the Newborn with its Mother in the Perspective of the Multiprofessional Team. Journal of Nursing UFPE On Line, 11(1), 94-101. doi:10.5205/reuol.9978-88449-6-1101201712.

Kommers, D., Oei, G., Chen, W., Feijs, L., \& Oetomo, S. B. (2016). Suboptimal bonding impairs hormonal, epigenetic and neuronal development in preterm infants, but these impairments can be reversed. Acta Paediatrica, 105, 738751. doi:10.1111/apa.13254.

Koopman, I., Callaghan-Koru, J. A., Alaofin, O., Argani, C. H., \& Farzin, A. (2016). Early Skin-To-Skin Contact for Healthy Full-Term Infants After Vaginal and Caesarean Delivery: A Qualitative Study on Clinician Perspectives. Journal of Clinical Nursing, 25, 1367-1376. doi:10.1111/jocn.13227.

Krumer-Nevo, M. (2009). From Voice to Knowledge: Participatory Action Research, Inclusive Debate and Feminism. International Journal of Qualitative Studies in Education, 22(3), 279-295. doi:10.1080/09518390902835462.

Landzelius, K. (2006). The Incubation of a Social Movement? Preterm Babies, Parent Activists, and Neonatal Productions in the US Context. Social Science \& Medicine, 62, 668-682. doi:10.1016/j.socscimed.2005.06.024

Langlois, S., Goudreau, J., \& Lalonde, L. (2014). Scientific Rigour and Innovations in Participatory Action Research Investigating Workplace Learning in Continuing Interprofessional Education. Journal of Inteprofessional Care, 28(3), 226-231. doi:10.3109/13561820.2014.885003.

Lapum, J., Federicks, S., Beanlands, H., McCay, E., Schwind, J., \& Romaniuk, D. (2012). A Cyborg Ontology in Health Care: Traversing into The Liminal Space Between Technology and Person-Centred Practice. Nursing Philosophy, 13, 276-288.

Lassi, Z. S., Middleton, P. F., Crowther, C. \& Bhutta, Z. A. (2015). Interventions to Improve Neonatal Health and Later Survival: An Overview of Systematic Reviews. EBioMedicine 2(985-1000). doi:10.1016/j.ebiom.2015.05.023.

Lavoie, J. G., Boulton, A. F., Gervais, L. (2012). Regionalization as an Opportunity for Meaningful Indigenous Participation in Healthcare: Comparing Canada and New Zealand. The International Indigenous Policy Journal, 3(1). doi: 10.18584/iipj.2012.3.1.2.

Lawn, J. E., Kinney, M. V., Belizan, J. M., Mason, E. M., McDougall, L., Larson, J., Howson, C. P. (2013). Born Too Soon: Accelerating actions for prevention and care of 15 million newborns born too soon. Reproductive Health, 10(Suppl 1)(S6).

Lawn, J. E., Mwansa-Kambafwile, J., Horta, B. L., Barros, F. C., \& Cousens, S. (2010). 'Kangaroo Mother Care' To Prevent Neonatal Deaths Due to Preterm Birth Complications. International Journal of Epidemiology, 39, i144-i154. doi:10.1093/ije/dyq031. 
Lee, I., Norr, K. F., \& Oh, K. (2005). Emotional Adjustment and Concerns of Korean Mothers of Premature Infants. International Journal of Nursing Studies, 42, 21-29. doi: 10.1016/j.ijnurstu.2004.05.004.

Lee, S-Y., \& Weiss. (2009). When East Meets West: Intensive Care Unit Experiences Among First-Generation Chinese American Parents. Journal of Nursing Scholarship, 41(3), 268-275. doi: 10.1111/j.15475069.2009.01290.x.

Lester, B. M., Hawes, K., Abar, B., Sullivan, M., Miller, R., Bigsby, R., Laptook, A., Salisbury, A., Taub, M., Lagasse, L. L., \& Padbury, J. R. (2014). Singlefamily Room Care and Neurobehavioral and Medical Outcomes in Preterm Infants. Pediatrics, 134, 754-760. doi: 10.1542/peds.2013-4252.

Levine, S. (2005). Developmental Determinants of Sensitivity and Resistance to Stress. Psychoneuroendocrinology, 30, 939-946.

Lim, S. (2018). Neonatal Nurses' Perceptions of Supportive Factors and Barriers to the Implemementation of Skin-To-Skin Care in Extremely Low Birth Weight (ELBW) Infants - A Qualitative Study. Journal of Neonatal Nursing, 24, 3943. https://doi.org/10.1016/j.jnn.2017.11.010.

Liu, W. F., Laudert, S., Perkins, B., MacMillan-York, E., Martin, S., Graven, S. (2007). The Development of Potentially Better Practices to Support the Neurodevelopment of Infants in the NICU. Journal of Perinatology, 27, S48S74.

Lopez-Maestro, M., Sierra-Garcia, P., Diaz-Gonzalez, C., Torres-Valdivieso, M. J., Lora-Pablos, D., Ares-Segura, S., \& Pallas-Alonso, C. R. (2017). Quality of Attachment in Infants Less Than $1500 \mathrm{~g}$ or Less than 32 Weeks. Related factors. Early Human Development, 104, 1-6.

Lorenz, L., Dawson, J. A., Jones, H., Jacobs, S. E., Cheong, J. L., Donath, S. M., Kamlin, C. O. F. (2017). Skin-to-skin Care in Preterm Infants Receiving Respiratory Support Does Not Lead to Physiological Instability. Archives of Disease in Childhood. Fetal Neonatal Edition, 102, F339-F344. doi:10.1136/archdischild-2016-311752

Lothian, J. A. (2001). Back to the Future: Trusting Birth. Journal of Perinatal and Neonatal Nursing, . 15(3), 13-22.

Ludington-Hoe, S. M., Ferreira, C., Swinth, J., \& Ceccardi, J. J. (2003). Safe Criteria and Procedure for Kangaroo Care With Intubated Preterm Infants. Journal of Obstetric, Gynecologic \& Neonatal Nursing, 32, 579-588. doi:10.1177/0884217503257618.

Luong, K. C., Nguyen, T. L., Thi, H. H. D., Carrara, H. P, O., \& Bergman, N. J. (2016). Newly Born Low Birthweight Infants Stabilise Better in Skin-ToSkin Contact than When Separated From Their Mothers: A Randomised Controlled Trial. Acta Paediatrica, 105, 381-390. doi:10.1111/apa.13164.

Lykes, M. B. (2013). Participatory and Action Research as a Transformative Praxis: Responding to Humanitarian Crises From the Margins. American Psychologist, November, 774-783. doi:10.1037/a0034360.

MacDorman, M. F., Declercq, E., \& Zhang, J. (2010). Obstetrical Intervention and the Singleton Preterm Birth Rate in the United States from 1991-2006. American Journal of Public Health, 100(11), 2241-2247. doi:10.2105/AJPH.2009.180570.

MacDonald, C. (2012). Understanding Participatory Action Research: A Qualitative Research Methodology Option. Canadian Journal of Action Research, 13(2), 34-50. 
Maddalena, P. (2013). Long Term Outcomes of Preterm Birth: The Role of Epigenetics. Newborn \& Infant Nursing Reviews(13), 137-139.

Maghaireh, D. F. A., Abdullah, K. L., Chan, C. M., Piaw, C. Y., \& Kawafha, M. M. A. (2016). Systematic Review of Qualitative Studies Exploring Parental Experiences in the Neonatal Intensive Care Unit. Journal of Clinical Nursing, 25, 2745-2756. doi:10.1111/jocn.13259.

Maguire, P. (1987). DOING PARTICIPATORY RESEARCH: A Feminist Approach. Amherst, Massachusetts, United States of America: The Center for International Education, University of Massachusetts.

Maitre, N. L., Key, A. P., Chorna, O. D., Slaughter, J. C., Matusz, P. J., Wallace, M. T., \& Murray, M. M. (2017). The Dual Nature of Early-Life Experience on Somatosensory Processing in the Human Infant Brain. Current Biology, 27, 1048-1054. http://dx.doi.org/10.1016/j.cub.2017.02.036.

Manson, L. (2012). Racism Compromises Māori Health. Kai Tiaki Nursing New Zealand, 18(3), 30.

Marcellus, L., \& Cross, S. (2016). Trauma-informed Care in the NICU: Implications for Early Childhood Development. Neonatal Network, 35(6), 359-366.

Marcum, J. A. (2008). Reflections on Humanizing Biomedicine. Perspectives in Biology and Medicine, 51(3), 392-405.

Marini, M. G., Chesi, P., Bruscagnin, M., Ceccatelli, M., \& Ruzzon, E. (2018). Digits and Narratives of the Experience of Italian Families Facing Premature Births. The Journal of Maternal-Fetal \& Neonatal Medicine, 31(17), 22582264. https://doi.org/10.1080/14767058.2017.1339272

McLellan, E., MacQueen, K. M., \& Neidig, J. L. (2003). Beyond the Qualitative Interview: Data Preparation and Transcription. Field Methods, 15(63), 63-84. doi:10.1177/1525822X02239573.

McCloskey, R. (2008). A guide to discourse analysis. Nurse Researcher, 16(1), 2444.

McEwen, B. S. (2007). Physiology and Neurobiology of Stress and Adaptation: Central Role of the Brain. Physiology Reviews, 87, 873-904. doi:10.1152/physrev.00041.2006.

McEwen, B. S. (2016). In Pursuit of Resilience: Stress, Epigenetics, and Brain Plasticity. Annals of the New York Academy of Sciences, 1363, 56-64. doi:10.1111/nyas.13020.

McGrath, J. M., Cone, S., \& Samra, H. A. (2011). Neuroprotection in the Preterm Infant: Further Understanding of the Short- and Long-term Implications for Brain Development. Newborn \& Infant Nursing Reviews, 11(3), 109-112. doi:10.1053/j.nainr.2011.07.002.

McGrath, J. M., Samra, H. A., \& Kenner, C. (2011). Family-centered Developmental Care Practices and Research. What Will the Next Century Bring? The Journal of Perinatal \& Neonatal Nursing, 25(2), 165-170. doi: 10.1097/JPN.0b013e1821a6706.

Meaney, M. J., \& Szyf, M. (2005). Maternal Care as a Model for ExperienceDependent Chromatin Plasticity? Trends in Neurosciences, 28(9), 456-463.

Meleis, A. I. (2016). Interprofessional Education: A Summary of Reports and Barriers to Recommendations. Journal of Nursing Scholarship, 48(1), 106112. doi: 10.1111/jnu.12184.

Memo, L., Longo, G., \& Soriani, P. (2011). Care Procedures for Healthy Term Newborn in Maternity Ward: The "Open" Nursery. Early Human Development, 875, S87-S88. doi:10.1016/j.earlhumdev.2011.01.038. 
Meyer, J. (2000). Qualitative Research in Health Care: Using Qualitative Methods in Health Related Action Research. British Medical Journal, 320, 178-181.

Miele, M. J. O., Pacagnella, R. C., Osis, M. J. D., Angelini, C. R., Souza, J. L., Cecatti, J. G. (2018). "Babies Born Early?" - Silences About Prematurity and Their Consequences. Reproductive Health, 15(154). https://doi.org/10.1186/s12978-018-0594-4.

Milette, I., Martel, M.-J., da Silva, M. R., \& McNeil, M. C. (2017a). Guidelines for the Institutional Implementation of Developmental Neuroprotective Care in the Neonatal Intensive Care Unit. Part A: Background and Rationale. A Joint Position Statement From the CANN, CAPWHN, NANN, and COINN. Canadian Journal of Nursing Research, 49(2). doi:10.1177/0844562117706882.

Milette, I., Martel, M.-J., da Silva, M. R., \& McNeil, M. C. (2017b). Guidelines for the Institutional Implementation of Developmental Neuroprotective Care in the NICU. Part B: Recommendations and Justification. A joint Position Statement From the CANN, CAPWHN, NANN, AND COINN. Canadian Journal of Nursing Research, 49(2), 63-74. doi:10.1177/0844562117708126.

Milgrom, J., Newnham, C., Anderson, P., J., Doyle, L., W., Gemmill, A., W., Lee, K., Inder, T. (2010). Early Sensitivity Training for Parents of Preterm Infants: Impact on the Developing Brain. Pediatric Research, 67(3), 330-335.

Mill, J. E., \& Ogilvie, L. D. (2003). Establishing Methodological Rigour in International Qualitative Nursing Research: A Case Study From Ghana. Methodological Issues In Nursing Research, 41(1), 80-87.

Ministry of Health. (2005). A Review of Neonatal Intensive Care Provision in New Zealand, February 2004. Ministry of Health (MOH), Wellington, New Zealand.

Ministry of Health. (2017). Report on Maternity 2015. Retrieved from https://www.health.govt.nz/system/files/documents/publications/report-onmaternity-2015-updated_12122017.pdf.

Ministry of Health. (2015). Report on Maternity 2014. Retrieved from https://www.health.govt.nz/system/files/documents/publications/report-onmaternity-2014-dec15.pdf.

Montirosso, R., Giusti, L., Del Prete, A., Zanini, R., Bellu, R., Borgatti, R., \& NEOACQUA Study Group. (2016). Does Quality Of Developmental Care in Nicus Affect Health-Related Quality of Life in 5-Y-Old Children Born Preterm? Pediatric Research. doi:10.1038/pr.2016.158.

Montirosso, R., \& Provenzi, L. (2015). Implications of Epigenetics and Stress Regulation on Research and Developmental Care of Preterm Infants. JOGNN, 44(2), 174-182.

Moore, E. R., Bergman, N., Anderson, G. C., \& Medley, N. (2016). Early Skin-ToSkin Contact for Mothers and Their Healthy Newborn Infants. Cochrane Database of Systematic Reviews, 11. doi:10.1002/14651858.CD003519.pub4.

Moore, H. (2015). Improving Kangaroo Care Policy And Implementation in the Neonatal Intensive Care. Journal of Neonatal Nursing, 21, 157-160.

Moore, T. A., Berger, A. M., \& Wilson, M. E. (2014). A New Way of Thinking About Complications of Permaturity. Biological Research for Nursing, 15(1), 72-82. doi:10.1177/1099800412461563.

Morad, S., Parry-Smith, W., \& McSherry, W. (2013). Dignity in Maternity Care. Evidence Based Midwifery, 11(2), 67-70. 
Morelius, E., \& Anderson, G. C. (2015). Neonatal Nurses' Beliefs About Almost Continuous Parent-Infant Skin-to-skin Contact in Neonatal Intensive Care. Journal of Clinical Nursing, 24, 2620-2627.

Morelius, E., Ortenstrand, A., Theodorsson, E., \& Frostell, A. (2015). A Randomised Trial of Continuous Skin-to-skin Contact After Preterm Birth and the Effects on Salivary Cortisol, Parental Stress, Depression, And Breastfeeding. Early Human Development, 91, 63-70. doi:10.1016/j.earlhumdev.2014.12.005.

Morgan, B. (2013). Biological Embedding Of Early Childhood Adversity: Toxic Stress and the Vicious Cycle of Poverty in South Africa. Res Policy Brief, Series 2.

Morse, J. M., Barrett, M., Mayan, M., Olson, K., \& Spiers, J. (2002). Verification Strategies for Establishing Reliability and Validity in Qualitative Research. International Journal Of Qualtitative Methods, 1(2), 13-22.

Moxon, S. G., Lawn, J, E., Dickson, K. E., Simen-Kapeu, A., Gupta, G., Deorari, A., Blencowe, H. (2015). Inpatient Care of Small and Sick Newborns: A MultiCountry Analysis of Health System Bottlenecks and Potential Solutions. BMC Pregnancy and Childbirth, 15(Suppl 2). doi:10.1186/1471-2393-15-S2S7.

Moynihan, R. (2011). Power to the people: Could a new and informed citizens' movement make medicine healthier? BMJ, 342. doi:10.1136/bmj.d2002.

Nairn, R., Pega, F., McCranor, T., Rankine, J., \& Barnes, A. (2006). Media, Racism and Public Health Psychology. Journal of Health Psychology, 11(2), 183196. doi: $10.1177 / 1359105306061179$.

Narvaez, D., Panksepp, J., Schore, A. N., \& Gleason, T. R. (2013). The Value of Using an Evolutionary Framework for Gauging Children's Well-Being. Evolution, early experience and human development: from research to practice and policy. University Press Scholarship Online: Oxford Scholarship Online.

Nazareth, I. V., Santos, I. M. M. (2014). Sociocultural Dimension of Parents of Premature Infants Discharged from a Neonatal Intensive Care Unit. Revista da Rede de Enfermagem do Nordeste, 15(4), 621-30. doi: 10.15253/21756783.201400040009.

Neczypor, J. L., \& Holley, S. L. (2017). Providing Evidence-Based Care During the Golden Hour. Nursing for Women's Health, 21(6), 462-472. http://dx.doi.org/10.1016/j.nwh.2017.10.011.

Newnham, D., Pincombe, J., \& McKellar, L. (2013). Access or Egress? Questioning the "Ethics" Of Ethics Committee Review for an Ethnographic Doctoral Research Study In A Childbirth Setting. International Journal of Doctoral Studies, 8, 121-136.

Niela-Vilen, H., Axelin, A., Melender, H.-L., \& Slantera, S. (2015). Aiming to be a Breastfeeding Mother in a Neonatal Intensive Care Unit and at Home: A Thematic Analysis of Peer-Support Group Discussion in Social Media. Maternal \& Child Nutrition, 11, 712-726. doi: 10.1111/mcn.12108.

Niela-Vilen, H., Feeley, N., \& Axelin, A. (2017). Hospital Routines Promote ParentInfant Closeness and Cause Separation in the Birthing Unit in the First 2 Hours After Birth: A Pilot Study. BIRTH, 44, 167-172. doi:10.1111/birt.12279.

Noergaard, B., Johannessen, H., Fenger-Gron, J., Kofoed, P.-E., \& Ammentorp, J. (2016). Participatory Action Research in the Field of Neonatal Intensive 
Care: Developing An Intervention to Meet the Fathers' Needs. A Case Study. Journal of Public Health Research, 5(744), 122-129.

Nosarti, C., Reichenberg, A., Murray, R. M., Cnattingius, S., Lambe, M. P., Yin, L., Hultman, C. M. (2012). Preterm Birth and Psychiatric Disorders in Young Adult Life. Archives of General Psychiatry, e1-e8. doi:10.1001/archgenpsychiatry.2011.1374.

Nugus, P., Greenfield, D., Travaglia, J., \& Braithwaite, J. (2012). The Politics Of Action Research: "If You Don't Like the Way Things are Going, Get Off the Bus". Social Science \& Medicine, 75, 1946-1953. http://dx.doi.org/10.1016/j.socscimed.2012.06.024.

Nyqvist, K. H., Anderson, G., Bergman, N., Cattaneo, A., Charpak, N., Davanzo, R., Widstrom, A.-M. (2010a). Towards Universal Kangaroo Mother Care: Recommendations and Report from the First European Conference and Seventh International Workshop on Kangaroo Mother Care. Acta Paediatrica, 99, 820-826.

Nyqvist, K. H., Anderson, G. C., Bergman, N., Cattaneo, A., Charpak, N., Davanzo, R., Widstrom, A.-M. (2010b). State of the Art and Recommendations. Kangaroo Mother Care: Application in a High-Tech Environment. Acta Paediatrica, 99, 812-819.

Nyqvist, K. H. (2016). Given the Benefits of Kangaroo Mother Care, Why Has its Routine Uptake Been So Slow? Acta Paediatrica, 105, 341-342. doi:10.1111/apa.13236.

Nyqvist, K. H., Haggkvist, A.-P., Hansen, M. N., Kylberg, E., Frandsen, A. L., Ragnhild, M., Haiek, L. N. (2013). Expansion of the Baby-Friendly Hospital Initiative Ten Steps to Successful Breastfeeding into Neonatal Intensive Care: Expert Group Recommendations. Journal of Human Lactation, 29(3), 300-309.

Nyqvist, K. H., \& Heinemann, A.-B. (2011). Kangaroo Mother Care: Optimal Support of Preterm Infants' Transition to Extra-Uterine Life in the High Tech NICU Environment. Current Women's Health Reviews, 7(3), 278-287.

Nystrom, K., \& Axelsson, K. (2002). Mothers' Experience of Being Separated From Their Newborns. Journal of Obstetric, Gynecologic \& Neonatal Nursing, 31(3), 275-282.

Obeidat, H., Bond, E., \& Callister, L. (2009). The Parental Experience of Having an Infant in the Newborn Intensive Care Unit. The Journal of Perinatal Education, 18(3), 23-29, doi: 10.1624/105812409X461199.

O'Connor, T. (2016). Involving Parents in the Care of Neonates. Kai Tiaki Nursing New Zealand, 4(22), 12-13.

Oda, K., \& Rameka, M., (2012). Students' Corner: Using Te Tiriti O Waitangi to Identify and Address Racism, and Achieve Cultural Safety in Nursing. Contemporary Nurse, 43(1), 107-112.

Oen, G., \& Stormark, K. M. (2013). Participatory Action Research in the Implementing Process of Evidence-Based Intervention to Prevent Childhood Obesity: Project Design of the "Healthy Future" Study. Journal of Obesity, 2013. http://dx.doi.org/10.1155/2013/437206.

Ohlsson, A., \& Jacobs, S. E. (2013). NIDCAP: A Systematic Review and Metaanalyses of Randomized Controlled Trials. Pediatrics, 131(3), e881-e893. doi:10.1542/peds.2012-2121.

Olshansky, E., Sacco, D., Braxter, B., Dodge, P., Hughes, E., Ondeck, M., Upvall, M. J. (2005). Participatory Action Research to Understand and Reduce 
Health Disparities. Nursing Outlook, 53, 121-126.

doi:10.1016/j.outlook.2005.03.002.

Pack, S., Tuffin, K., \& Lyons, A. (2016). Reducing Racism Against Māori in Aotearoa New Zealand. New Zealand Journal of Psychology, 45(3).

Papps, E., \& Olssen, M. (1997). Doctoring Childbirth and Regulating Midwifery in New Zealand: A Foucauldian Perspective. Palmerston North: Dunmore Press.

Patterson, J. E., \& Vakili, S. (2014). Relationships, Environment, and the Brain: How Emerging Research is Changing What We Know about the Impact of Families on Human Development. Family Process, 53, 22-32. doi:10.1111/famp.1205.

Pease, B. (2002). Rethinking Empowerment: A Postmodern Reappraisal for Emancipatory Practice. British Journal of Social Work, 32, 135-147.

Penn, S. (2015). Overcoming the Barriers to Using Kangaroo Care in Neonatal Settings. Nursing Children and Young People, 27(5), 22-27.

Pennestri, M.-H., Gaudreau, H., Bouvette-Turcot, A.-A., Moss, E., Lecompte, V., Atkinson, L., Lydon, J., Steiner, M., Meaney, M. J. (2015). Attachment Disorganization Among Children in Neonatal Intensive Care Unit: Preliminary results. Early Human Development, 91, 601-606.

Penrod, J., Loeb, S. J., Ladonne, R. A., Martin, L. M. (2016). Empowering Change Agents in Hierarchical Organizations: Participatory Action Research in Prisons. Research in Nursing \& Health, 39(3), 142-153. doi:10.1002/nur.21716.

Perinatal and Maternal Mortality Review Committee (2018). Twelfth Annual Report of the Perinatal and Maternal Mortality Review Committee: Reporting Mortality 2016. Health Quality \& Safety Commission, Wellington, New Zealand. Retrieved from http://www.hqsc.govt.nz/ourprogrammes $/ \mathrm{mrc} / \mathrm{pmmrc}$.

Perry, B. D. (2009). Examining Child Maltreatment Through a Neurodevelopmental Lens: Clinical Applications of the Neurosequential Model of Therapeutics. Journal of Loss and Trauma, 14, 240-255. doi:10.1080/15325020903004350.

Perry, R. E., Blair, C., \& Sullivan, R. M. (2017). Neurobiology of Infant Attachment: Attachment Despite Adversity and Parental Programming Of Emotionality. Current Opinion in Psychology, 17, 1-6.

Peters, D. H., Adam, T., Alonge, O., Agyepong, I. A., Tran, N. (2013). Implementation Research: What It Is and How To Do It. British Medical Journal, 347. doi: 10.1136/bmj.f6753

Phillips, R. (2013). The Sacred Hour: Uninterrupted Skin-to-Skin Contact Immediately After Birth. Newborn \& Infant Nursing Reviews, 13, 67-72.

Philpott-Robinson, K., Lane, S. J., Korostenski, L., \& Lane, A. E. (2017). The Impact of the Neonatal Intensive Care Unit on Sensory and Developmental Outcomes in Infants Born Preterm: A Scoping Review. British Journal of Occupational Therapy, 80(8), 459-469. doi:10.1177/0308022617709761.

Pierrat, V., Goubet, N., Peifer, K., \& Sizun, J. (2007). How Can We Evaluate Developmental Care Practices Prior to their Implementation in a Neonatal Intensive Care Unit? Early Human Development, 83, 415-418. doi:10.1016/j.earlhumdev.2007.03.003.

Pihama, L. (2010). He Kākano i Ruia Mai i Rangātea: Māori Whānau Stories of Neonatal Intensive Care Units. (Report 08-RF-08). Retrieved from www.maramatanga.co.nz/projects. 
Pineda, R. G., Neil, J., Dierker, D., Smyser, C. D., Wallendorf, M., Kidokoro, H., Inder, T. (2014). Alterations in Brain Structure and Neurodevelopmental Outcome in Preterm Infants Hospitalized in Different Neonatal Intensive Care Unit Environments. The Journal of Pediatrics, 162, 52-60.

Pineda, R. ., Stransky, K. E., Rogers, C., Duncan, M. H., Smith, G. C., Neil, J., \& Iner, T. (2012). The Single Patient Room in the NICU: Maternal and Family Effects. Journal of Perinatology 32(7). 545-551. doi: 10.1038/jp.2011.144.

PMMRC. (2018). Twelfth Annual Report of the Perinatal and Maternal Mortality Review Committee: Reporting Mortality 2016. Wellington: Health Quality \& Safety Commission. Retrieved from http://www.hqsc.govt.nz/ourprogrammes $/ \mathrm{mrc} / \mathrm{pmmrc}$.

Ponterotto, J., G. (2005). Qualitative Research in Counseling Psychology: A Primer on Research Paradigms and Philosophy of Science. Journal of Counseling Psychology, 52(2), 126-136. doi: 10.1037/0022-0167.52.2.126

Porges, S. W. (2015). Making the World Safe for our Children: Down-regulating Defence and Up-regulating Social Engagement to 'Optimise' the Human Experience. Children Australia, 40(2), 114-123. doi:10.1017/cha.2015.12.

Porges, S. W., \& Furman, S. A. (2011). The Early Development of the Autonomic Nervous System Provides a Neural Platform for Social Behavior: A Polyvagal Perspective. Infant Child Dev, 20(1), 106-118. doi:10.1002/icd.688.

Price, P., \& Miner, S. (2008). Extraordinarily Ordinary Moments of Co-occupation in a Neonatal Intensive Care Unit. Occupation, Participation and Health 29(2), 72-78.

Pridham, K., Limbo, R., Schroeder, M., Krolikowski, M., \& Henriques, J. (2006). A Continuing Education Program for Hospital and Public Health Nurses to Guide Families of Very Low Birth-weight Infants in Caregiving. The Journal of Continuing Education in Nursing, 37(2), 74-85.

Pritchard, V. E., \& Montgomery-Honger, A. (2014). A Comparison of Parent and Staff Perceptions of Setting-specific and Everyday Stressors Encountered by Parents with Very Preterm Infants Experiencing Neonatal Intensive Care. Early Human Development, 90, 549-555. http://dx.doi.org/10.1016/j.earlhumdev.2014.07.006.

Probst, B. (2016). Both/and: Researcher as Participant in Qualitative Inquiry. Qualitative Research Journal, 16(2), 149-158. https://doi.org/10.1108/QRJ06-2015-0038.

Provenzi, L., Barello, S., \& Graffigna, G. (2015). Caregiver Engagement in the Neonatal Intensive Care Unit: Parental Needs, Engagement Milestones, and Action Priorities for Neonatal Healthcare of Preterm Infants. In Patient Engagement: A consumer-centred model to innovate healthcare. De Gruyter. doi 10.1515/9783110452440-009.

Provenzi, L., \& Montirosso, R. (2015). "Epigenethics" in the Neonatal Intensive Care Unit. Conveying Complexity in Health Care for Preterm Children. JAMA Pediatrics. doi:10.1001/jamapediatrics.2015.43.

Putnam, K. T., Harris, W. W., \& Putnam, F. W. (2013). Synergistic Childhood Adversities and Complex Adult Psychopathology. Journal of Traumatic Stress, 26, 435-442. doi:10.1002/jts.21833.

Pynoos, R. S., Steinberg, A. M., Layne, C. M., Liang, L.-J., Briggs, E., Habib, M., Fairbank, J. A. (2014). Modeling Constellations of Trauma Exposure in the 
National Child Traumatic Stress Network Core Data Set. Psychological Trauma: Theory, Research, Practice, and Policy, 6(S1), S9-S17.

Reason, P. (1998). Political, Epistemological, Ecological and Spiritual Dimensions of Participation. Studies in Cultures, Organizations and Societies, 4, 147167.

Redshaw, S. (2014). 'She's Done Two and That's Harsh': The Agency of Infants with Congenital Conditions as Invoked Through Parent Narratives. Health Sociology Review, 23(2), 125-135.

Reid, J., Cormack, D., \& Crowe, M. (2016). The Significance of Socially-assigned Ethnicity for Self-identified Māori Accessing and Engaging with Primary Healthcare in New Zealand. Health, 20(2), 143-160. doi: $10.1177 / 1363459315568919$.

Reiger, K., \& Morton, C. (2012). Standardizing or Individualizing? A Critical Analysis of the "Discursive Imaginaries" Shaping Maternity Care Reform. INTERNATIONAL JOURNAL OF CHILDBIRTH, 2(3), 173-186. http://dx.doi.org/10.1891/2156-5287.2.3.173.

Reynolds, L. C., Duncan, M. M., Smith, G. C., Mathur, A., Neill, J., Inder, T., \& Pineda, R. G. (2013). Parental Presence and Holding In The Neonatal Intensive Care Unit and Associations With Early Neurobehavior. Journal of Perinatology, 33, 636-641.

Ribeiro, C., Moura, C., Sequeira, C., Barbieri, M., \& Erdmann, A. (2015). Parents' and Nurses' Perceptions of Nursing Care in Neonatology - an Integrative Review. Revista de Enfermagem Referencia 4(4), 137-146.

Richardson, F. (2012). Editorial: Cultural Safety 20 Years On. Time to Celebrate or Commiserate? Whitireia Nursing Journal, 19, 5-8.

Richardson, F., \& MacGibbon, L. (2010). Cultural Safety: Nurses' Accounts of Negotiating the Order of Things. Women's Studies Journal, 24(2), 54-65.

Richardson, S. (2004). Aoteaoroa/New Zealand Nursing: from Eugenics to Cultural Safety. Nursing Inquiry 11(1), 35-42.

Rick, S. L. (2006). Developmental Care on Newborn Intensive Care Units: Nurses' Experiences and Neurodevelopmental, Behavioural, And Parenting Outcomes. A Critical Review of The Literature. Journal of Neonatal Nursing, 12, 56-61. doi:10.1016/j.jnn.2006.01.004.

Roberts, C. (2008). RELATING SIMPLY? Feminist Encounters with Techonoscience in the Early Twenty-first Century. Australian Feminist Studies, 23(55), 75-86. doi:10.1080/08164640701816249.

Roberts, K. L., Paynter, C., \& McEwan, B. (2000). A Comparison of Kangaroo Mother Care and Conventional Cuddling Care. Neonatal Network, 19(4), 3135.

Robertson, K. (2009). Becoming a facilitator: Tips for the uninitiated. The Canadian Nurse, 105(8), 6-7.

Robinson, O. C. (2014). Sampling in Interview-Based Qualitative Research: A Theoretical and Practical Guide. Qualitatvie Research in Psychology, 11(1), 25-41. https://doi.org/10.1080/14780887.2013.801543.

Robiquet, P., Amamiara, P.-E., Rakza, T., Deruelle, P., Mestdagh, B., Blondel, G., Subtil, D. (2016). Observation of Skin-to-Skin Contact and Analysis of Factors Linked to Failure to Breastfeed Within 2 Hours After Birth. Breastfeeding Medicine, 11(3), 126-132. doi:10.1089/bfm.2015.0160. 
Rook, M. H. (2017). Living Nursing Values: A Collective Case Study. Doctor of Philosophy in Nursing, Victoria Universtiy of Wellington, Wellington, New Zealand. Retrieved from http://hdl.handle.net/10063/6358.

Rossman, B., Greene, M. M., Dratovil, A. L., \& Meier, P. P. (2017). Resilience in Mothers of Very-Low-Birth-Weight Infants Hospitalized in the NICU. JOGNN, 46, 434-445. http://dx.doi.org/10.1016/j.jogn.2016.11.016.

Rothfield, P. (2002). Feminism and Medicine. Internal Medicine Journal, 32, 320321.

Roue, J.-M., Kuhn, P., Lopez Maestro, M., Maastrup, R. A., Mitanchez, D., Westrup, B., \& Sizun, J. (2017). Eight Principles for Patient-Centred and Family-Centred Care for Newborns in the Neonatal Intensive Care Unit. Archives of Diseases in Childhood. Fetal and Neonatal Edition, 102, F364F368. doi:10.1136/archdischild-2016-312180.

Roy, A., \& Ryba, T. V. (2012). Curb your Enthusiasm: A Case of Young Malaysian Women's Emotional Embodiment. Women in Sport and Physical Activity Journal, 21(1).

Ruiz, J. G., Charpak, N., Castillo, M., Bernal, A., Rios, J., Trujillo, T., \& Cordoba, M. A. (2016). Economic Evaluation of Kangaroo Mother Care: Cost Utility Analysis of Results from a Randomized Controlled Trial Conducted In Bogota. Journal of Clinical Epidemiology, 1-10. http://dx.doi.org/10.1016/j.jclinepi.2016.10.007.

Russell, K., Weaver, B., \& Vogel, R., L. (2015). Neuroprotective Core Measure 2: Partnering with Families - Effects of a Weighted Mternally-Scented Parental Simulation Device on Premature Infants in Neonatal Intensive Care. Newborn \& Infant Nursing Reviews, 15(3), 97-103. doi:10.1053/j.nainr.2015.06.005.

Rutgers, S. G., \& Meyers, S. (2015). Skin-to-skin Contact. Journal of Alternative Medical Research, 7(3), 185-191.

Ryan, K. M., \& Grace, V. M. (2001). Medicalization and Women's Knowledge: The Construction of Understandings of Infant Feeding Experiences in Post-WW II New Zealand. Health Care for Women International, 22, 483-500.

Samra, H. A., McGrath, J. M., Wehbe, M., \& Clapper, J. (2012). Epigenetics and Family-Centered Developmental Care for the Preterm Infant. Advances in Neonatal Care, 12(5S), S2-S9. doi:10.1097/ANC.0b013e318265b4bd.

Sanders, M. R., \& Hall, S. L. (2018). Trauma-informed Care in the Newborn Intensive Care Unit: Promoting Safety, Security and Connectedness. Journal of Perinatology, 38(1), 3-10. doi:10.1038/jp.2017.124.

Schenk, L. K. (2005). Mothering an Extremely Low Birth Weight Infant. Doctor of Philosophy in Nursing, University of Mississippi, Mississippi, United States of America.

Schore, A., \& McIntosh, J. (2011). Family Law and the Neuroscience of Attachment, Part 1. Family Court Review, 49(3), 501-512.

Scott, N. (2014). A Māori Cultural Reluctance to Present for Care, or a Systems and Quality Failure? How we Pose the Issue, Informs our Solutions. The New Zealand Medical Journal, 127(1393), 8-11.

Seidman, G., Unnikrishnan, S., Kenny, E., Myslinski, S., Cairns-Smith, S., Mulligan, B., \& Engmann, B. (2015). Barriers and Enablers of Kangaroo Mother Care Practice: A Systematic Review. PLoS ONE, 10(5). doi:10.1371/journal.pone.0125643. 
Seneviratne, S., Campbell, I., Scott, N., Shirley, R., Peni, T., Lawrenson, R. (2015). Ethnic Differences in Breast Cancer Survival in New Zealand: Contributions of Differences in Screening, Treatment, Tumor Biology, Demographics and Comorbidities. Cancer Causes Control, 26, 1813-1824.

Sharma, D., Murki, S., \& Oleti, T. P. (2016a). To Compare Cost Effectiveness of 'Kangaroo Ward Care' with 'Intermediate Intensive Care' in Stable Very Low Birth Weight Infants (Birth Weight < 1100 Grams): A Randomized Control

Trial. Italian Journal of Pediatrics, 42(64). doi:10.1186/s13052-016-0274-3.

Sharma, D., Murki, S., \& Pratap, O. T. (2016b). The Effect of Kangaroo Ward Care in Comparison with "Intermediate Intensive Care" on the Growth Velocity in Preterm Infant with Birth Weight<1100g: Randomized Control Trial. European Journal of Pediatics, 175, 1317-1324. doi:10.1007/s00431-0162766-y.

Shonkoff, J. P. (2010). Building a New Biodevelopmental Framework to Guide the Future of Early Childhood Policy. Child Development, 81(1), 357-367.

Shonkoff, J. P. (2016). Capitalizing on Advances in Science to Reduce the Health Consequesnce of Early Childhood Adversity. JAMA Pediatrics, 170(10), 1003-1007. doi:10.1001/jamapediatrics.2016.1559.

Shonkoff, J. P., Garner, A. S., The Committee on Psychosocial Aspects of Child and Family Health, Committee on Early Childhood, Adoption, and Dependent Care, \& Section on Developmental and Behavioral Pediatrics. (2012). The Lifelong Effects of Early Childhood Adversity and Toxic Stress. Pediatrics, 129(1), e233-e246. doi:10.1542/peds.2011-2663.

Skene, C., Gerrish, K., Price, F., Pilling, E., \& Bayliss, P. (2015). Developing Family-Centred Care in a Neonatal Intensive Care Unit: An Action Research Study Protocol. Journal of Advanced Nursing, 72(3), 658-668. doi:10.1111/jan.12863.

Small, S. A. (1995). Action-Oriented Research: Models and Methods. Journal of Marriage and Family, 57(4), 941-955.

Smith, E. R., Bergelson, I., Constantian, S., Valsangkar, B., \& Chan, G. J. (2017). Barriers and Enablers Of Health System Adoption of Kangaroo Mother Care: A Systemic Review of Caregiver Perspectives. BMC Pediatrics, 17(35). doi:10.1186/s12887-016-0769-5.

Solomons, N., \& Rosant, C. (2012). Knowledge and Attitudes of Nursing Staff and Mothers Towards Kangaroo Mother Care in the Eastern Sub-District Of Cape Town. South Africal Journal of Clinical Nutrition, 25(1), 33-39.

Soni, A., Amin, A., Pater, D. V., Fahey, N., Shah, N., Phatak, A. G., Nimbalkar, S. M. (2016). The Presence of Physician Champions Improved Kangaroo Mother Care in Rural Western India. Acta Paediatrica, 105, e390-e395. doi:10.1111/apa.13445.

Spence, D. B. (2005). Hermeneutic Notions Augment Cultural Safety Education. Cultural Safety 44(9), 409-414.

Spinelli, M., Frigerio, A., Montali, L., Fasolo, M., Spada, M. S., \& Mangili, G. (2016). 'I Still Have Difficulties Feeling Like a Mother': The Transition to Motherhood of Preterm Infants Mothers. Psychology \& Health, 31(2), 184204. http:/dx.doi.org/10.1080/08870446.2015.1088015.

Spitzer, D. L. (2004). In Visible Bodies: Minority Women, Nurses, Time, and the New Economy of Care. Medical Anthropology Quarterly, 18(4), 490-508. 
Springer, R. A., \& Clinton, M. E. (2015). Doing Foucault: Inquiring into Nursing Knowledge with Foucauldian Discourse Analysis. Nursing Philosophy, 16, 87-97. doi:10.1111/nup.12079.

Stainton, C., Prentice, M., Lindrea, K. B., Wise, S., \& Dando, H. (2001). Evolving a Developmental Care Culture in a Neonatal Intensive Care Nursery. An Action Research Project. Neonatal, Paediatric and Child Health Nursing, 4(3), 6-14.

Stark, M. A., Eremynse, M., \& Zwelling, E. (2016). Importance of the Birth Environment to Support Physiologic Birth. JOGNN, 45, 285-294. http://dx.doi.org/10.1016/j.jogn.2015.12.008.

Stephens, C. (2011). Narrative Analysis in Health Psychology Research: Personal, Dialogical and Social Stories of Health. Health Psychology Review, 5(1), 6278. doi:10.1080/17437199.2010.543385.

Stephens, C., \& Breheny, M. (2013). Narrative Analysis in Psychological Research: An Integrated Approach to Interpreting Stories. Qualitative Research in Psychology, 10(1), 14-27. doi:10.1080/14780887.2011.586103.

Stoner, L., Pager, R., Matheson, A., Tarrant, M., Stoner, K., Rubin, D., \& Perry, L. (2015). The Indigenous Health Gap: Raising Awareness and Changing Attitudes. Perspectives in Public Health, 135(2), 68-70. doi: 1177/1757913915569965.

Swain, J. E., Kim, P., Spicer, J., Ho, S. S., Dayton, C. J., Almadih, A., \& Abel, K. M. (2014). Approaching the Biology of Human Parental Attachment: Brain Imagaing, Oxytocin and Coordinated Assessments of Mothers and Fathers. Brain Research, 1580, 78-101. http://dx.doi.org/10.1016/j.brainres.2014.03.007.

Syed, S. B., Dadwal, V., \& Martin, G. (2013). Reverse Innovation in Global Health Systems: Towards Global Innovation Flow. Globalization and Health, 9(36).

Syed, S. B., Dadwal, V., Rutter, P., Storr, J., Hightower, J. D., Gooden, R., Pittet, D. (2012). Developed-developing Country Partnerships: Benefits to Developed Countries? Globalization and Health, 8(17).

Symington, A. J., \& Pinelli, J. (2006). Developmental Care for Promoting Development and Preventing Morbidity in Preterm Infants. Cochrane Database of Systematic Reviews(2). doi:10.1002/14651858.

Tallandini, M., A., \& Scalembra, C. (2006). Kangaroo Mother Care and MotherPremature Infant Dyadic Interaction. Infant Mental Health Journal, 27(3), 251-275. doi:10.1002/imhj.20091.

Taussig, K.-S., Hoeyer, K., \& Helmreich, S. (2013). The Anthropology of Potentiality in Biomedicine an Introduction to Supplement 7. Current Anthropology, 54(S7), S3-S14.

ten Ham, W., Minnie, K. C. S., \& van der Walt, C. S. J. C. (2016). Health Care Professionals' Perspectives on the Requirements Facilitating The Roll-Out Of Kangaroo Mother Care In South Africa. Health SA Gesondheid, 21, 228-237.

Theunissen, C. E. (2011). The Nurse's Role in Improving Health Disparities Experienced by the Indigenous Māori of New Zealand. Contemporary Nurse, 39(2), 281-286. doi: 10.1572/conu.2011.39.2.281.

Thomas, J. C., Letourneau, N., Bryce, C. I., Campbell, T. S., Giesbrecht, G. F., \& The AprON Study Team. (2017). Biological Embedding of Perinatal Social Relationships in Infant Stress Reactivity. Developmental Psychobiology, 59, 425-435. doi:10.1002/dev.21505. 
Thompson, K. (2009). Māori Whānau Experiences of a Neonatal Intensive Care Unit: Waikato Hospital. Thesis, Master Of Social Sciences in Psychology. The Universtiy of Waikato, Hamilton, New Zealand. Retrieved from https://hdl.handle.net/10289/3587.

Thomson, G., Moran, V. H., Axelin, A., Dykes, F., \& Flacking, R. (2013). Integrating a Sense of Coherence into the Neonatal Environment. BMC Pediatrics, 13(84).

Torre, M. E., \& Ayala, J. (2009). Envisioning Participatory Action research: Entremundos. Feminism \& Psychology, 19(3), 387-393. doi:10.1177/0959353509105630.

Traynor, M. (2010). Professional Autonomy in 21st Century Healthcare: Nurses' Accounts of Clinical Decision-Making. Social Science \& Medicine, 71, 15061512. doi:10.1016/j.socscimed.2010.07.029.

Tsigos, C., \& Chrousos, G. P. (2002). Hypothalamic-pituitary-adrenal axis, Neuroendocrine Factors and Stress. Journal of Psychosomatic Research, 53, 865-871.

Turecki, G., \& Meaney, M. J. (2016). Effects of the Social Environment and Stress on Glucocorticoid Receptor Gene Methylation: A Systematic Review. Biological Psychiatry, 79, 87-96.

Uwaezuoke, S. N. (2017). Kangaroo Mother Care in Resource-Limited Settings: Implementation, Health Benefits, and Cost-Effectiveness. Research and Reports in Neonatology, 7, 11-18.

VandenBerg, K. A. (2007). Individualized Developmental Care for High Risk Newborns in the NICU: A Practice Guideline. Early Human Development, 83, 433-442. doi:10.1016/j.earlhumdev.2007.03.008.

Vanderbilt, D., \& Gleason, M. M. (2011). Mental Health Concerns of the Premature Infant Through the Lifespan. Pediatrics Clinics of North America, 58, 815832. doi:10.1016/j.pcl.2011.06.012.

Vanderveen, J. A., Bassler, D., Robertson, C. M. T., \& Kirpalani, H. (2009). Early Interventions Involving Parents to Improve Neurodevelopmental Outcomes of Premature Infants: A Meta-Analysis. Journal of Perinatology, 29, 343351.

van Rooyen, D., Nomgqokwana, S. L., Kotze, W. J., \& Carlson, S. (2006). Guidelines to Facilitate the Nursing Accompaniment of Mothers With Infants in a Neonatal Intensive Care Unit. Health SA Gesondheid, 11(2), 3-12.

Ventegodt, S., \& Merrick, J. (2011). Biomedicine in General Practice. Journal of Alternative Medical Research, 3(4), 393-404.

Vesel, L., Bergh, A.-M., Kerber, K. J., Valsangkar, B., Mazia, G., Moxon, S. G., Blencowe, H., Darmstadt, G. L. de Graft Johnson, J., Dickson, K. E., Ruiz Pelaez, J. G., von Xylander, S. R., Lawn, J. E. (2015). Kangaroo Mother Care: A Multi-Country Analysis of Health System Bottlenecks and Potential Solutions. BMC Pregnancy and Childbirth, 15(Suppl 2).

Victoria University of Wellington, New Zealand \& Governance Policy Group (2009). Treaty of Waitangi Statute. Retrieved from https://www.victoria.ac.nz/documents/policy/governance/treaty-of-waitangistatute.pdf.

Vohr, B., McGowan, E., McKinley, L., Tucker, R., Keszler, L., \& Alksninis, B. (2017). Differential Effects of the Single-Family Room Neonatal Intensive Care Unit on 18-24-Month Bayley Scores of Preterm Infants. The Journal of Pediatrics 185, 42-48. http://dx.doi.org10.1016/j.jpeds.2017.01.056. 
Walker, R. C., Walker, S., Morton, R. L., Tong, A., Howard, K., Palmer, S. C. (2017). Māori Patients' Experiences and Perspectives of Chronic Kidney Disearse: a New Zealand Qualitative Interview Study. BMJ Open, 7, e013829. doi: 10.1136/bmjopen-2016-013829.

Wallin, L., \& Eriksson, M. (2009). Newborn Individual Development Care and Assessment Program (NIDCAP): A Systematic Review of the Literature. Worldviews on Evidence-Based Nursing, 6(2), 54-69.

Wallin, L., Rudberg, A., \& Gunningberg, L. (2005). Staff Experiencesi Implementing Guidelines for Kangaroo Mother Care - A Qualitative Study. International Journal of Nursing Studies, 42, 61-73.

Watson, G. (2010). Parental Liminality: a Way of Understanding the Early Experiences of Parents Who Have a Very Preterm Infant. Journal of Clinical Nursing, 20, 1462-1471. doi: 10.1111/j.1365-2702.2010.03311.x.

Weatherston, D. J., \& Browne, J. V. (2016). What is Infant Mental Health and Why is it Important for High-risk Infants and Their Families? Newborn \& Infant Nursing Reviews, 16, 259-263. http://dx.doi.org/10.1053/j.nainr.2016.09.026.

Weedon, C. (1997). Feminist Practice and Poststructuralist theory ( $2^{\text {nd }}$ Ed.). Oxford: Blackwell Publishers.

Weiss, E. M., Barg, F. K., Black, E., \& Joffe, S. (2016). Parental Decision-Making Preferences in Neonatal Intensive Care. The Journal of Pediatrics, 179, 3641. http://dx.doi.org10.1016/j.jpeds.2016.08.030.

Welch, M. G., Firestein, M. R., Austin, J., Hane, A. A., Stark, R. I., Hofer, M. A., Myers, M. M. (2015). Family Nurture Intervention in the Neonatal Intensive Care Unit Improves Social-Relatedness, Attention, and Neurodevelopment of Preterm Infants at 18 Months in a Randomized Controlled Trial. Journal of Child Psychology and Psychiatry, 56(11). doi:10.1111/jcpp.12405.

Welch, M. G., Hofer, M. A., Stark, I., Andrews, H. F., Austin, J., Glickstein, S. B., Ludwig, R. J., Myers, M. M., \& the FNI Trial Group. (2013). Randomized Controlled Trial of Family Nurture Intervention in the NICU: Assessments of Length of Stay, Feasibility and Safety. BNC Pediatrics, 13(148).

Wetherell, M., Taylor, S., \& Yates, S. J. (2001). Discourse as Data. A guide for Analysis. Milton Keynes, Sage Publications Ltd.

White, R. D. (2011). The Newborn Intensive Care Unit Environment of Care: How We Got Here, Where We're Headed, and Why. Seminars in Perinatology, 35, 2-7.

White, R. D. (2014). Development of Care in the NICU. Journal of Perinatology, $34,174-175$.

Widstrom, A.-M., Lilja, G., Aaltomaa-Michalias, P., Dahllof, A., Lintula, M., \& Nissen, E. (2011). Newborn Behaviour to Locate the Breast When Skin-toskin: A Possible Method for Enabling Early Self-Regulation. Acta Paediatrica, 100, 79-85. doi:10.1111/j.1651-2227.2010.01983.x.

Wigert, H., Johansson, R., Berg, M., \& Hellstrom, A. L. (2006). Mothers' Experiences of Having Their Newborn Child in a Neonatal Intensive Care Unit. Scandenavian Journal of Caring Sciences, 20, 35-41.

Williamson, K. (2006). Research in Construtivist Frameworks Using Ethnographic Techniques. Library Trends 55(1), 81-101.

Wilson, D. (2008). The Significance of a Culturally Appropriate Health Service for Indigenous Māori Women. Contemporary Nurse 28(1-2), 173-188. doi: 10.5172/conu.673.28.1-2.173. 
Wilson, D., \& Baker, M. (2012). Bridging Two Worlds: Māori Mental Health Nursing. Qualitative Health Research, 22(8)., 1073-1082. doi: 10.11.77/1049732312450213.

Wilson, N. W. (2012). Chaos in Western Medicine: How Issues of SocialProfessional Status are Undermining Our Health. Global Journal of Health Science, 4(6). doi:10.5539/gjhs.v4n6p1.

Woodward, L. J., Bora, S., Clark, C. A. C., Mongomery-Honger, A., Pritchard, V. E., Spencer, C., \& Austin, N. C. (2014). Very Preterm Birth: Maternal Experiences of the Neonatal Intensive Care Unit. Journal of Perinatology, 34(7). doi: 10.1038/jp.2014.43.

World Association for Infant Mental Health. (2016). WAIMH Position Paper on the Rights of Infants. Perspectives in Infant Mental Health, Winter-Spring. Retrieved from https://perspectives.waimh.org/wpcontent/uploads/sites/9/2017/05/PositionPaperRightsInfants_May_13_2016_1-2_Perspectives_IMH_corr.pdf.

World Health Organization. (2006). Quality of Care: A Process for Making Strategic Choices in Health Systems. (ISBN 924156324 9). Geneva, Switzerland: WHO Library Cataloguing-in-Publication Data. Retrieved from https://www.who.int/management/quality/assurance/QualityCare_B.Def.pdf.

World Health Organization. (2015). WHO Recommendations on Interventions to Improve Preterm Birth Outcomes. (ISBN 978924 150898 8). Geneva, Switzerland: WHO Library Cataloguing-in-Publication Data. Retrieved from https://apps.who.int/iris/bitstream/handle/10665/183037/9789241508988_en g.pdf;jsessionid=E352D5E025284E81678BF0D97A814E3F? sequence=1.

World Health Organization. (2016). Standards for Improving Quality of Maternal and Newborn Care in Health Facilities. Geneva, Switzerland: WHO Library Cataloguing-in-Publication Data. Retrieved from https://apps.who.int/iris/bitstream/handle/10665/249155/9789241511216eng.pdf? sequence $=1$

World Health Organization: Western Pacific Region. (2014). Action Plan for Healthy Newborn Infants in the Western Pacific Region (2010-2014). Geneva, Switzerland: WHO Library Cataloguing-in-Publication Data. Retrieved from https://iris.wpro.who.int/bitstream/handle/10665.1/10454/9789290616634_en g.pdf.

Youngson, R. (2014). Re-inspiring Compassionate Caring: The Reawakening Purpose Workshop. Journal of Compassionate Health Care, 1(1), 1-3.

Youngson, R., \& Blennerhassett, M. (2016). Humanising Healthcare. British Medical Journal, 355(i6262). doi:10.1136/bmj.i6262.

Zambas, S. I., \& Wright, J. (2016). Impact of colonialism on Māori and Aboriginal Healthcare Access: a Discussion Paper. Contemporary Nurse, 52(4), 398409. http://dx.doi.org/10.1080/13076178.2016.1195238.

Zani, A. V., Tonete, V. L. P., Lima, C. M. G. P. (2014). Maternal Representations About the Provision of Care to Newborns at Risk: a Collective Discourse. Online Brazilian Journal of Nursing, 13(3), 321-331.

Zmyj, N., Witt, S., Almut, W., Neumann, H., \& Lucke, T. (2017). Social Cognition in Children Born Preterm: A Perspective on Future Research Directions. Frontiers in Psychology, 8(455). 